\title{
WestVirginiaUniversity
}

THE RESEARCH REPOSITORY @ WVU

Graduate Theses, Dissertations, and Problem Reports

2002

\section{Glass reinforcement of recycled polycarbonate}

Adam Al-Mulla

West Virginia University

Follow this and additional works at: https://researchrepository.wvu.edu/etd

\section{Recommended Citation}

Al-Mulla, Adam, "Glass reinforcement of recycled polycarbonate" (2002). Graduate Theses, Dissertations, and Problem Reports. 2615.

https://researchrepository.wvu.edu/etd/2615

This Dissertation is protected by copyright and/or related rights. It has been brought to you by the The Research Repository @ WVU with permission from the rights-holder(s). You are free to use this Dissertation in any way that is permitted by the copyright and related rights legislation that applies to your use. For other uses you must obtain permission from the rights-holder(s) directly, unless additional rights are indicated by a Creative Commons license in the record and/ or on the work itself. This Dissertation has been accepted for inclusion in WVU Graduate Theses, Dissertations, and Problem Reports collection by an authorized administrator of The Research Repository @ WVU.

For more information, please contact researchrepository@mail.wvu.edu. 


\title{
Glass Reinforcement of Recycled Polycarbonate
}

\author{
Adam Al-Mulla \\ Dissertation submitted to College of Engineering and Mineral Resources at \\ West Virginia University in partial fulfillment of the requirements of \\ Doctor of Philosophy \\ in \\ Chemical Engineering
}

\author{
Dr. Rakesh K. Gupta, Ph. D., Chair \\ Dr. John W. Zondlo, Ph. D. \\ Dr. Charter D. Stinespring, Ph. D. \\ Dr. Hota V. GangaRao, Ph. D. \\ Dr. Carl Irwin, Ph. D.
}

Department of Chemical Engineering

Morgantown, West Virginia

2002

Keywords: Polycarbonate, Recycling, Glass fiber, Mechanical properties, Rheology, Viscoelasticity, 


\title{
Abstract \\ Glass Reinforcement of Recycled Polycarbonate
}

\begin{abstract}
Adam Al-Mulla
The main barrier to recycling of post-consumer plastics is the commingled nature of these materials with consequent poor and variable mechanical, thermal and flow properties; separating the polymers by chemical type is prohibitively expensive and does not completely solve the problem of batch-to-batch variability in properties that results from variations in the nature and amount of impurities and variation in polymer molecular weight and molecular weight distribution. In the present work, it is proposed that the addition of up to $20 \mathrm{wt} \%$ short glass fibers to the mixed plastic makes the mechanical and flow properties of the composite depend more on the glass reinforcement than on the polymeric matrix. The truth of this hypothesis is demonstrated by providing data on polymers obtained from end-of-life electronics, typically polycarbonate (PC) and acrylonitrile-butadiene-styrene (ABS). It is shown that impact strength and elongation-tobreak in a tensile test are the two mechanical properties that are most sensitive to the presence of impurities in PC. On adding short glass fibers, these two properties become almost insensitive to changes in matrix composition provided that the matrix contains at least $75 \%$ virgin PC. Thus, a sample containing $15 \mathrm{wt} \%$ glass fibers has an impact strength of $1.4 \mathrm{ft}-1 \mathrm{~b} /$ in when the matrix contains recycled $\mathrm{PC}$, and this impact strength goes up to only $1.8 \mathrm{ft}-\mathrm{lb} /$ in when the matrix is entirely virgin PC; the increase in strain-atfracture is from $5 \%$ to $6.7 \%$. Similarly, the viscosity difference between PC melts with added glass fibers is acceptably small if the matrix contains at least $75 \%$ virgin PC. This says that, for purposes of recycling, separation of commingled plastics is not necessary, and one can formulate a "green" product having $25 \%$ recycled polymer (based on resin content) by the addition of an appropriate amount of short glass fibers.
\end{abstract}


Dedicated to my parents, wife Enas Salmeen and children Abdulla and Noura 


\section{Acknowledgements}

I would like to use this opportunity to thank everybody without whose help this work would have not been possible.

First of all, I would like to express my deep gratitude to my adviser Dr. Rakesh K. Gupta. His advice, support and encouragement were invaluable and indispensable many a times.

I am also thankful to my committee members- Dr. John W. Zondlo, Dr. John W. Zondlo, Dr. Charter D. Stinespring, Dr. Hota V. GangaRao, and Dr. Carl Irwin for their help and advice.

A special note of thanks to technician Mr. Jim Hall and office manager Ms. Linda Rogers for all their help on so many occasions.

I would like to express my gratitude to Kuwait University that made my study possible. This research was partially supported by the US Department of Energy through grant DE-FC26-00FT40598 for the project, "Research, commercialization, and workforce development in the polymer / electronics recycling industry."

Finally, my sincere thank to my family in Kuwait for all their support and encouragement over the years, without which none of this would be possible. 


\section{Table of Contents}

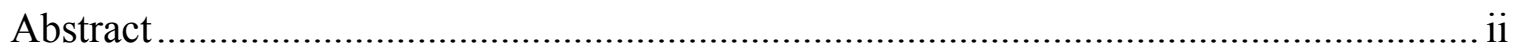

Acknowledgements .................................................................................................. iv

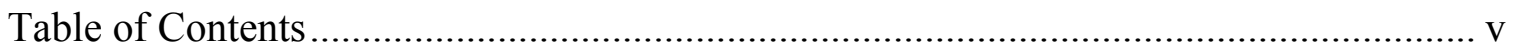

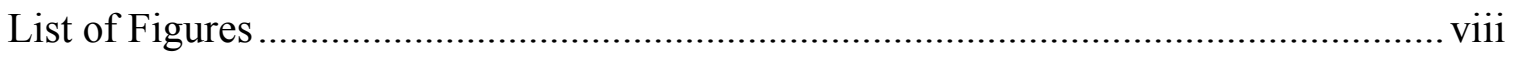

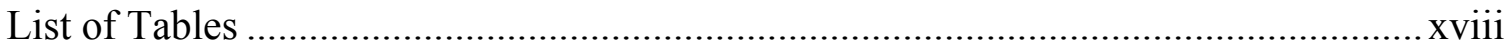

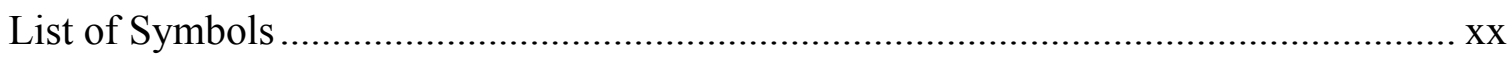

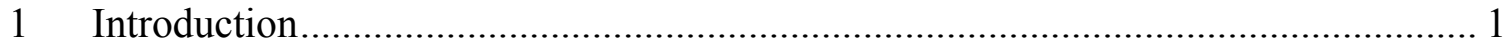

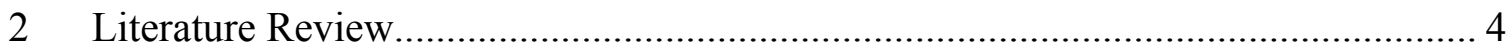

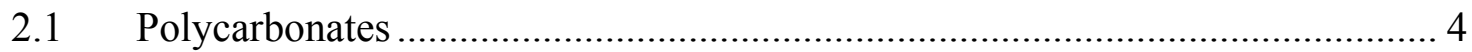

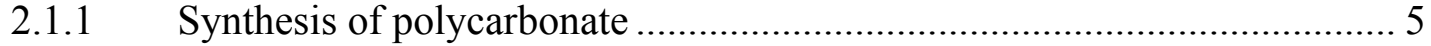

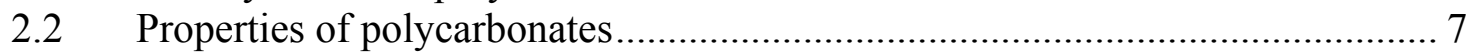

2.2.1 Molecular weights and molecular weight distributions ............................. 7

2.2.2 Stress-strain behavior........................................................................ 8

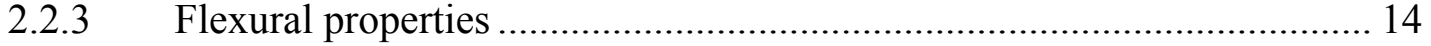

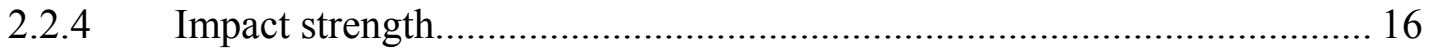

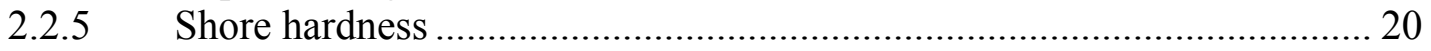

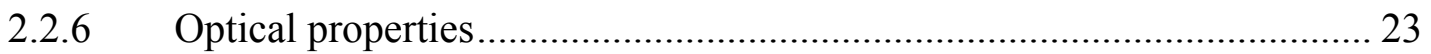

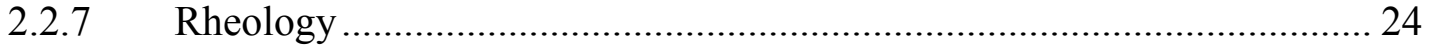

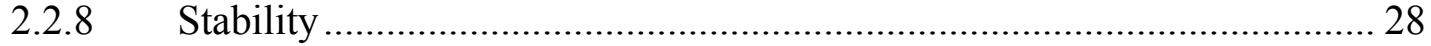

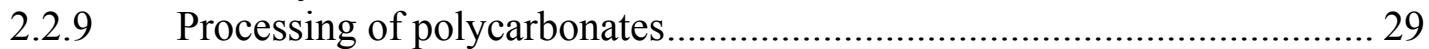

$2.3 \quad$ Applications of polycarbonates.................................................................. 30

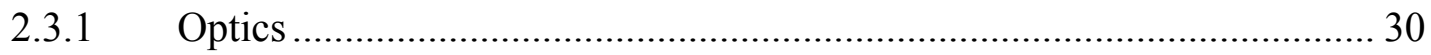

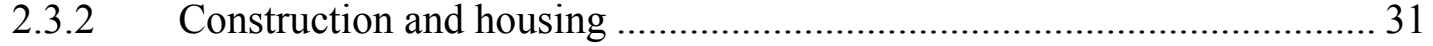

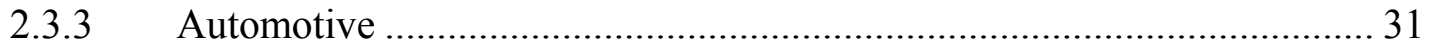

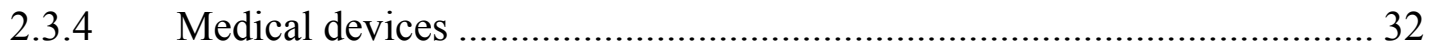

2.3.5 Appliances and consumer products ……………................................. 32

2.4 Modification of polycarbonates ……………….................................................. 33

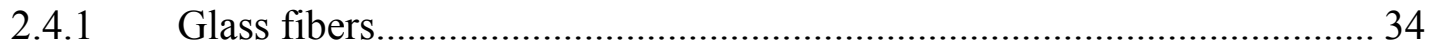

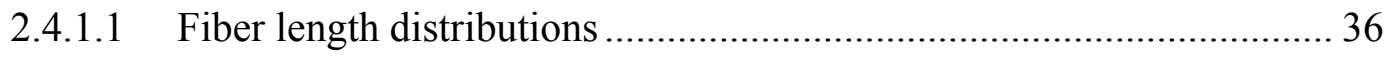

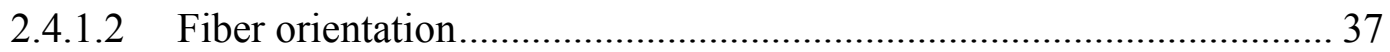




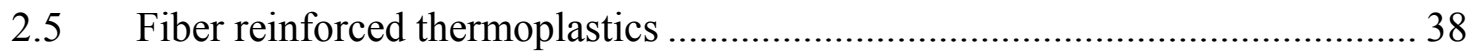

2.5.1 The stiffness of partially oriented composites ......................................... 41

2.5.2 Strength of short-fiber composites......................................................... 42

2.5.3 Strength of partially oriented composites …………………………......... 44

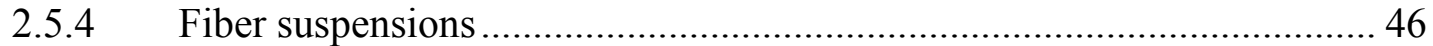

2.5.5 Dynamic mechanical analysis............................................................ 51

3 Purpose of this Study and Approach Taken............................................................ 55

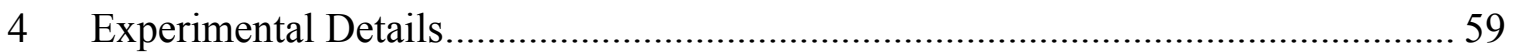

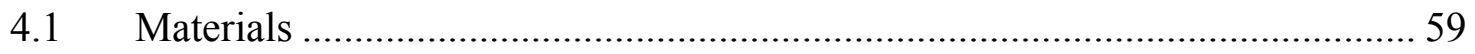

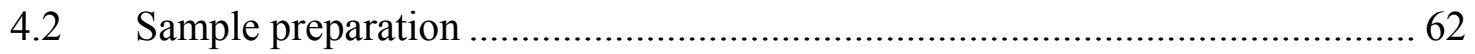

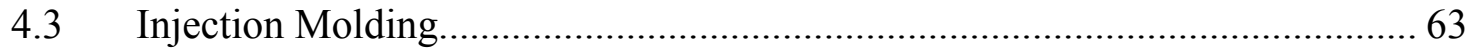

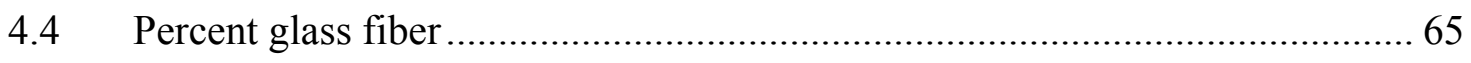

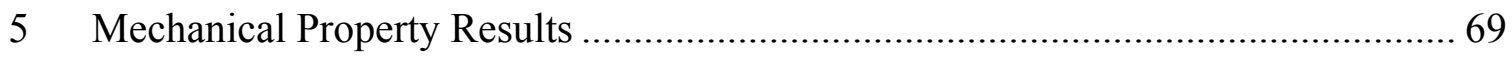

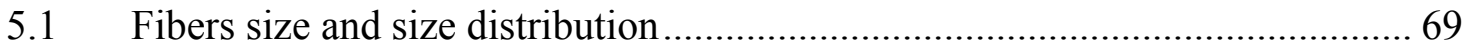

5.2 Mechanical behavior of the polycarbonate system......................................... 72

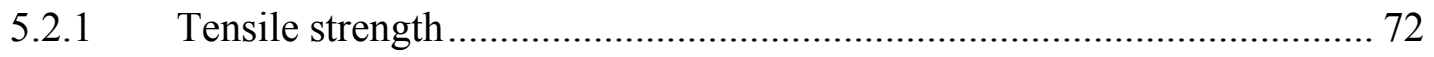

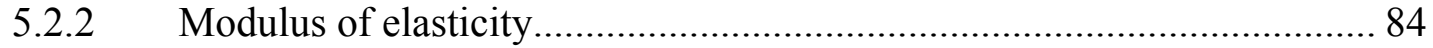

5.2.3 Elongation at yield and break ............................................................. 90

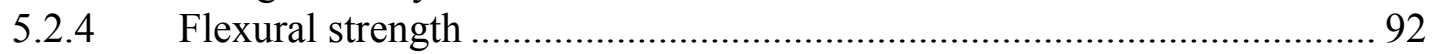

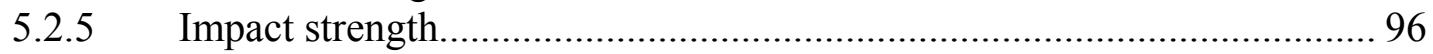

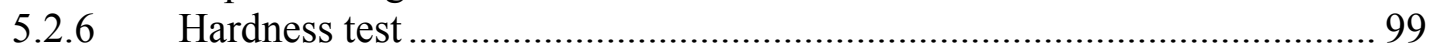

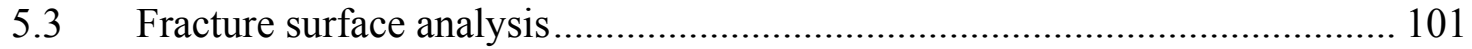

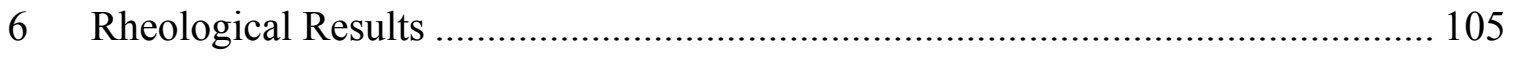

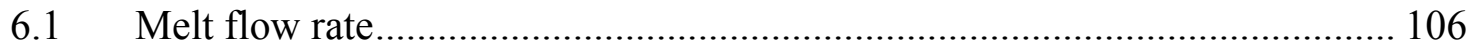

6.2 Shear viscosity using melt flow indexer ..................................................... 108

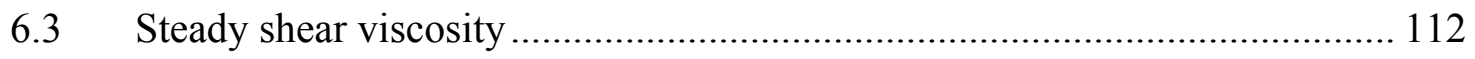

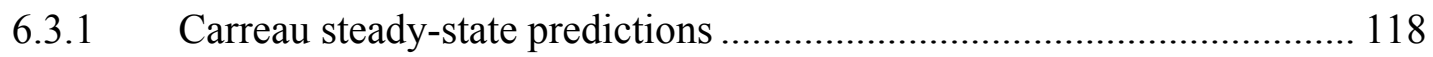

6.4 Dynamic testing results (samples are solid) ................................................... 122

6.5 Coefficient of thermal expansion.............................................................. 128

6.6 Dynamic testing results (samples are molten) ….......................................... 130

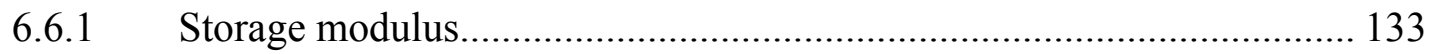

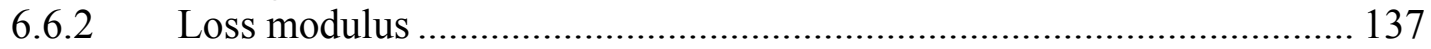

6.6.3 Prediction the storage and loss modulus ............................................. 141 


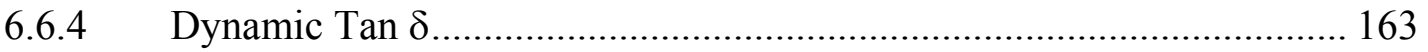

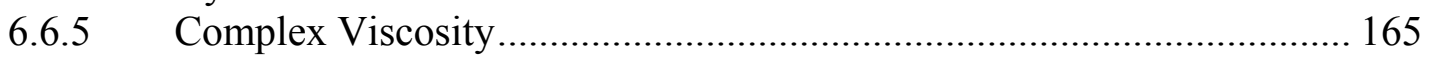

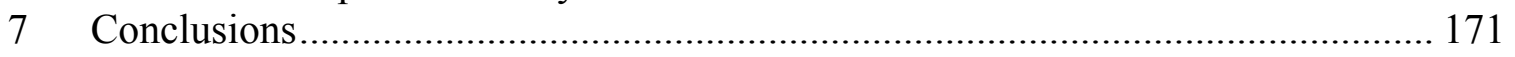

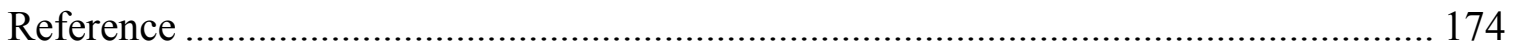

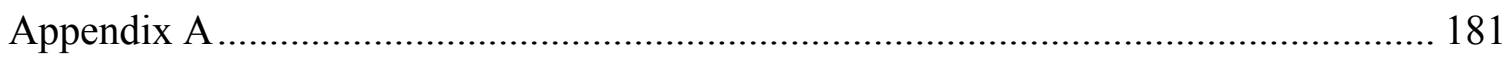

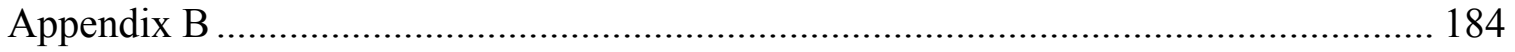

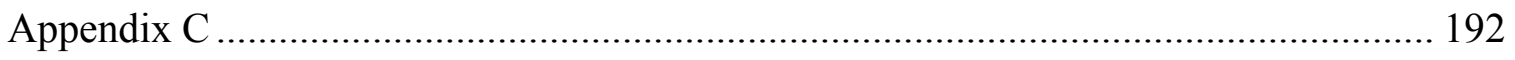

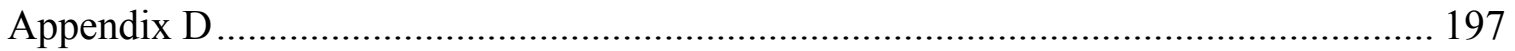

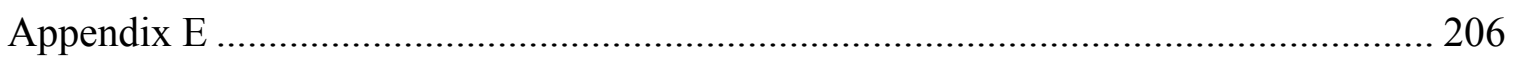

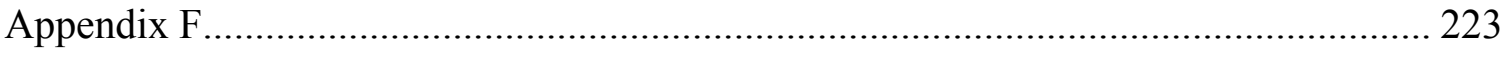




\section{List of Figures}

Figure 2-1 Bisphenol A Polycarbonate (PC) ............................................................ 5

Figure 2-2 Typical stress-strain curve for polycarbonate ........................................ 9

Figure 2-3 General types of stress-strain curves................................................ 14

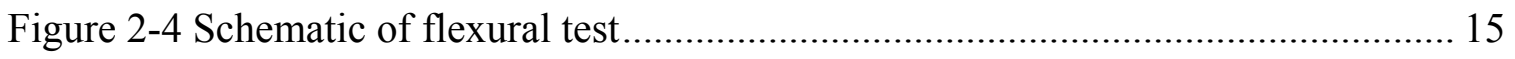

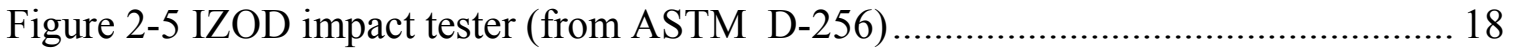

Figure 2-6 Mounting of IZOD impact specimen .................................................. 19

Figure 2-7 Notcher used to notches according to the ASTM standard.......................... 20

Figure 2-8 Schematic representation of the procedure of normal harness testing

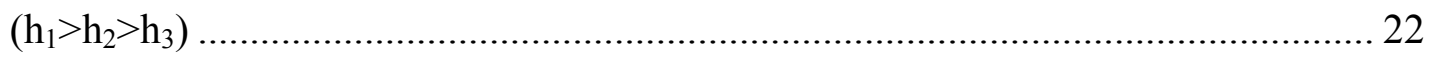

Figure 2-9 Schematic diagram of the melt flow index apparatus showing cross section

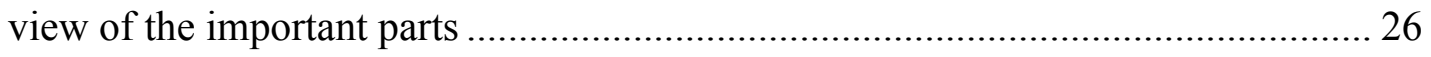

Figure 2-10 Characteristic flow curves of $37,000 \mathrm{~g} / \mathrm{mol}$ polycarbonate resin at various

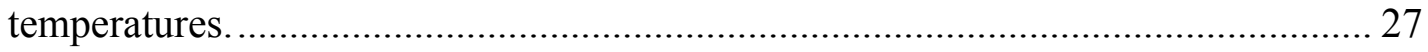

Figure 2-11 A compact disk made from Polycarbonate ............................................. 30

Figure 2-12 Schematic presentation of the changes in fiber orientation occurring during flow. (a) Initial random distribution, (b) rotation during shear flow and (c) alignment

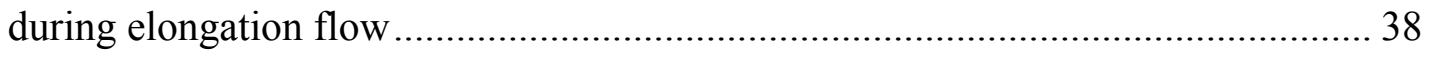

Figure 2-13 Definition of symbols used in Cox shear-lag analysis .............................. 43

Figure 2-14 Fiber spacing for dilute, semi-concentrated, and concentrated fibers.......... 48

Figure 2-15 Viscosity of un-reinforced polycarbonate (LEXAN ${ }^{*}$ HF1110) and glass reinforced polycarbonate $\left(\mathrm{LEXAN}^{*} 500\right)$. Taken from www.GEplastics.com........ 51 
Figure 2-16 Storage modulus (E') for Polycarbonate LEXAN HF1110 (test conducted at frequency equal to $1 \mathrm{~Hz}$ )

Figure 2-17 Storage modulus for un-reinforced polycarbonate and 10\% glass reinforced polycarbonate (test conducted at frequency equal to $1 \mathrm{~Hz}$ )

Figure 2-18 Loss modulus for un-reinforced polycarbonate and 10\% glass reinforced polycarbonate (test conducted at frequency equal to $1 \mathrm{~Hz}$ ) 54

Figure 3-1 Life of polycarbonate in computer housing 58

Figure 3-2 Method examined for improving the mechanical and rheological properties of recycled polycarbonate 58

Figure 4-1 Schematic of blending virgin polycarbonate with glass fiber 60

Figure 4-2 Schematic of blending recycled polycarbonate with glass fiber 61

Figure 4-3 Schematic of blending glass fiber with polycarbonate $(50 \%$ Virgin $+50 \%$ recycle) 61

Figure 4-4 Schematic of blending polycarbonate and impurities 61

Figure 4-5 Schematic of blending glass fiber with polycarbonate and impurities 62

Figure 4-6 Schematic diagram for twin screw extruder.. 63

Figure 4-7 Schematic diagram of injection molding 64

Figure 4-8 Injection molded samples.

Figure 4-9 schematic of the experimental tests conducted..... 67

Figure 4-10 schematic of the experimental tests conducted 68

Figure 5-1 A dispersed field of short glass fibers recovered from injection molded 10\% glass fiber and virgin polycarbonate 71 
Figure 5-2 Fiber length distribution for $10 \%$ GF and virgin polycarbonate.................... 72

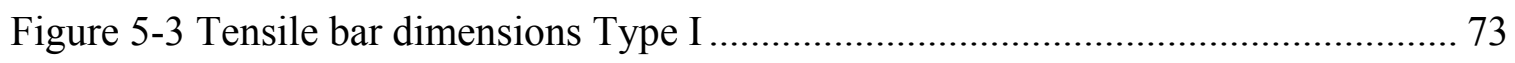

Figure 5-4 Instron machine used to measure the tensile and flexural strength................ 74

Figure 5-5 Stress strain curve for virgin PC containing glass fiber............................. 75

Figure 5-6 Stress-strain curve for un-reinforced and glass reinforced recycled PC ........ 76

Figure 5-7 Stress-strain curve for un-reinforced and glass reinforced 50\% recycled PC+

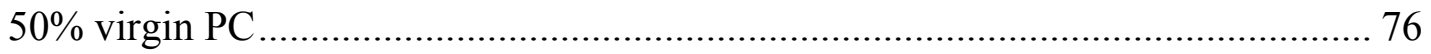

Figure 5-8 Effect of glass fiber on yield strength of polycarbonate ............................ 77

Figure 5-9 Effect of glass fiber on strength at fracture of polycarbonate ....................... 78

Figure 5-10 The tensile strength versus volume fraction of glass fibers for recycled PC 79 Figure 5-11 Effect of impurities on 15\% glass reinforced polycarbonate (impurities are

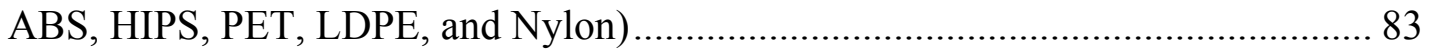

Figure 5-12 Using rules of mixture to predict the tensile strength of $15 \%$ glass reinforced

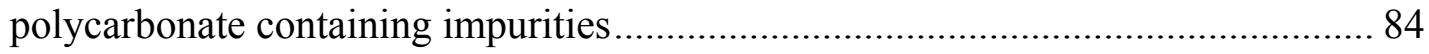

Figure 5-13 Effect of glass fiber on the modulus of elasticity .................................. 86

Figure 5-14 Effect of Impurities on the Modulus of Elasticity of 15\% glass reinforced polycarbonate 89

Figure 5-15 Effect of glass fiber on \% elongation at yield of polycarbonate ................. 91

Figure 5-16 Effect of glass fiber on \% elongation at break polycarbonate .................... 91

Figure 5-17 Influence of impurities on the ductility of $15 \%$ glass reinforced polycarbonate. 92

Figure 5-18 Direction of fibers along the direction of flow 93 


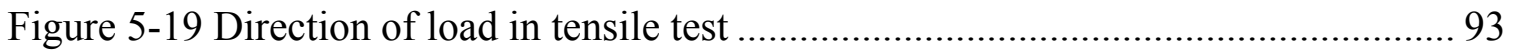

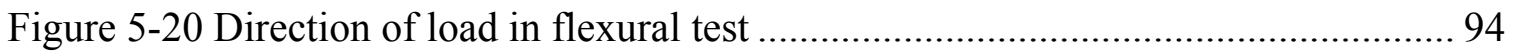

Figure 5-21 Flexural test sample dimensions ...................................................... 95

Figure 5-22 Effect of glass fiber on the flexural strength of polycarbonate ................... 95

Figure 5-23 Influence of impurities on the flexural strength of un-reinforced and 15\%

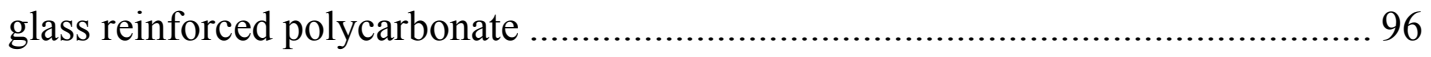

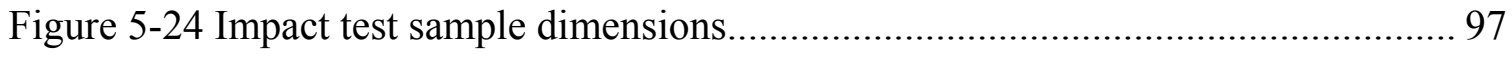

Figure 5-25 Effect of glass fiber on the impact strength of polycarbonate (samples are

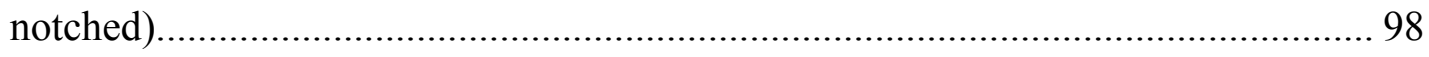

Figure 5-26 Effect of glass fiber content on impact strength of polycarbonate (samples

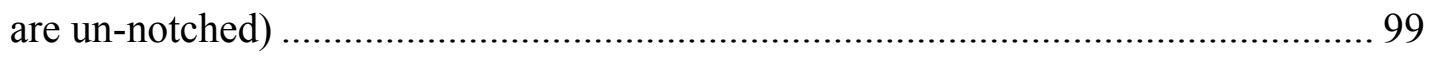

Figure 5-27 Influence of impurities on the notched impact test ..................................99

Figure 5-28 Durometer hardness used to measure the hardness of polycarbonate......... 101

Figure 5-29 Effect of glass fiber on Shore's hardness ........................................ 101

Figure 5-30 The fiber pull-out for 15\% glass fiber and mixture of virgin $\mathrm{PC}+$ recycled PC.

Figure 5-31 The alignment of glass fibers (15\% glass fiber and recycled PC) parallel to

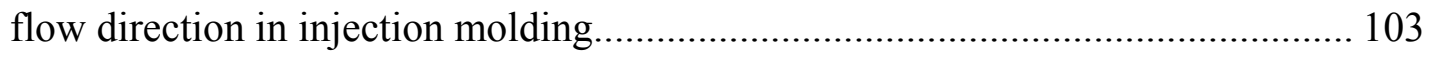

Figure 5-32 5\% glass fiber and virgin PC ductile fracture ...................................... 104

Figure 5-33 5\% glass fiber and recycled PC........................................................ 104

Figure 6-1 Influence of impurities on the melt flow index of virgin $\mathrm{PC}\left(\mathrm{T}=260{ }^{\circ} \mathrm{C}\right.$, load $=2.16 \mathrm{~kg})$ 108 
Figure 6-2 Effect of glass fiber on the shear viscosity of virgin polycarbonate 110

Figure 6-3 Effect of glass fiber on the shear viscosity of recycled polycarbonate 111

Figure 6-4 Effect of glass fiber on the shear viscosity of $50 \%$ virgin polycarbonate $+50 \%$ recycled polycarbonate

Figure 6-5 Parallel plate viscometer with two disks of radius R separated by a distance B, with dimensions such that $\mathrm{R}>>\mathrm{B}$.

Figure 6-6 Virgin polycarbonate steady-state viscosity at temperature $260{ }^{\circ} \mathrm{C}$. 115

Figure 6-7 Recycled polycarbonate steady-state viscosity at temperature $260{ }^{\circ} \mathrm{C}$ 115

Figure 6-8 50\% virgin polycarbonate $+50 \%$ recycled polycarbonate steady-state viscosity at temperature $260{ }^{\circ} \mathrm{C}$ 116

Figure 6-9 Unreinforced polycarbonate steady-state viscosity at temperature $260{ }^{\circ} \mathrm{C} \ldots 117$

Figure 6-10 20\% glass reinforced PC steady-state viscosity at temperature $260{ }^{\circ} \mathrm{C}$

Figure 6-11 Variation of relative viscosity at constant shear rate with glass fiber volume fraction for all three systems. 118

Figure 6-12 Virgin polycarbonate predicted steady state viscosity vs. shear rate using Carreau 120

Figure 6-13 Recycled polycarbonate predicted steady state viscosity vs. shear rate using Carreau. 121

Figure 6-14 50\% virgin polycarbonate $+50 \%$ recycled polycarbonate predicted steady state viscosity vs. shear rate using Carreau. 121

Figure 6-15 Storage modulus for unreinforced and glass reinforced virgin polycarbonate 123 
Figure 6-16 Storage modulus for unreinforced and glass reinforced recycled

polycarbonate

Figure 6-17 Storage modulus for unreinforced and glass reinforced 50\% virgin and 50\% recycled polycarbonate

Figure 6-18 Loss modulus for unreinforced and glass reinforced virgin polycarbonate 124

Figure 6-19 Loss modulus for unreinforced and glass reinforced recycled polycarbonate

Figure 6-20 Loss modulus for unreinforced and glass reinforced 50\% virgin and 50\% recycled polycarbonate

Figure 6-21 Storage modulus for unreinforced polycarbonate vs. temperature 126

Figure 6-22 Storage modulus for 20\% glass reinforced polycarbonate vs. temperature 127

Figure 6-23 Loss modulus for unreinforced polycarbonate vs. temperature..... 128

Figure 6-24 Loss modulus for 20\% glass reinforced polycarbonate vs. temperature.... 128

Figure 6-25 Effect of glass fiber on the coefficient of thermal of expansion.

Figure 6-26 Strain sweep for virgin polycarbonate at frequency equal $1 \mathrm{~Hz}$ and temperature $250{ }^{\circ} \mathrm{C}$

Figure 6-27 Virgin polycarbonate storage modulus vs. frequency $\left(\mathrm{T}=290^{\circ} \mathrm{C}\right) \ldots \ldots \ldots \ldots . . . .134$

Figure 6-28 Recycled polycarbonate storage modulus vs. frequency $\left(\mathrm{T}=290{ }^{\circ} \mathrm{C}\right) \ldots \ldots \ldots 134$

Figure $6-2950 \%$ virgin polycarbonate $+50 \%$ recycled polycarbonate storage modulus vs. frequency $\left(\mathrm{T}=290{ }^{\circ} \mathrm{C}\right)$ 135

Figure 6-30 Unfilled polycarbonate storage modulus vs. frequency $\left(\mathrm{T}=290^{\circ} \mathrm{C}\right)$. 135

Figure 6-31 20\% glass fiber reinforced polycarbonate storage modulus vs. frequency 


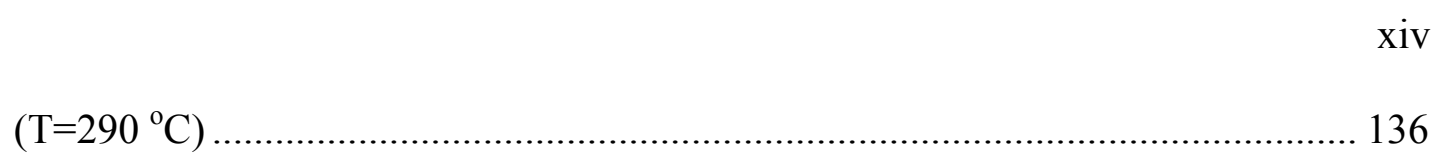

Figure 6-32 Influence of temperature on the storage modulus of $20 \%$ glass reinforced recycled PC

Figure 6-33 Virgin polycarbonate loss modulus vs. frequency $\left(\mathrm{T}=290^{\circ} \mathrm{C}\right) \ldots \ldots \ldots \ldots \ldots \ldots . . .138$

Figure 6-34 Recycled polycarbonate loss modulus vs. frequency $\left(\mathrm{T}=290{ }^{\circ} \mathrm{C}\right)$............. 138

Figure $6-3550 \%$ virgin polycarbonate $+50 \%$ recycled polycarbonate loss modulus vs. frequency $\left(\mathrm{T}=2900^{\circ} \mathrm{C}\right)$

Figure 6-36 Unfilled polycarbonate loss modulus vs. frequency $\left(\mathrm{T}=290{ }^{\circ} \mathrm{C}\right)$. 140

Figure 6-37 20\% glass fiber reinforced polycarbonate loss modulus vs. frequency ( $T=290$ $\left.{ }^{\circ} \mathrm{C}\right)$ 140

Figure 6-38 Influence of temperature on the loss modulus of $20 \%$ glass reinforced recycled PC 141

Figure 6-39 Mechanical analog of the generalized Maxwell model 143

Figure 6-40 The storage modulus G' MPa (unfilled circles) and the loss modulus G” MPa (filled circles) versus frequency $\omega \mathrm{rad} / \mathrm{s}$. Symbols: experimental data for virgin PC at $250{ }^{\circ} \mathrm{C}$. Solid lines: results of numerical simulation 147

Figure 6-41 The storage modulus G' MPa (unfilled circles) and the loss modulus G” MPa (filled circles) versus frequency $\omega \mathrm{rad} / \mathrm{s}$. Symbols: experimental data for virgin PC with 20 wt.\% of glass fibers at $290{ }^{\circ} \mathrm{C}$. Solid lines: results of numerical simulation 148

Figure 6-42 The shear modulus G' MPa versus the filler content $v$ wt.\%. Symbols: treatment of observations for virgin $\mathrm{PC}$ at $250{ }^{\circ} \mathrm{C}$ (unfilled circles) and $290{ }^{\circ} \mathrm{C}$ 
(filled circles). Solid lines: approximation of the experimental data by the function $\mathrm{G}=\mathrm{G}_{\mathrm{o}}+\mathrm{G}_{1}$ v. Curve $1: \mathrm{G}_{\mathrm{o}}=0.45, \mathrm{G}_{1}=8.92$ E10-3 curve $2: \mathrm{G}_{\mathrm{o}}=0.84, \mathrm{G} 1=-3.40 \mathrm{E}-2$ 150

Figure 6-43: The average activation energy $Z$ versus the filler content $v$ wt.- $\%$. Symbols: treatment of observations for virgin PC at $250{ }^{\circ} \mathrm{C}$ (unfilled circles) and $290{ }^{\circ} \mathrm{C}$ (filled circles). Solid lines: approximation of the experimental data by the function $\log Z=Z_{o}-Z_{l} v$. Curve $1: Z_{\mathrm{o}}=1.15 \mathrm{E}-1, Z_{1}=1.73 \mathrm{E}-2 ;$ curve $2: Z_{\mathrm{o}}=-1.06 \mathrm{E}-1, Z_{1}=$ 1.38E-1. 152

Figure 6-44: The attempt rate $\Gamma_{\mathrm{o}} \mathrm{s}^{-1}$ versus the filler content $v$ wt.-\%. Symbols: treatment of observations for virgin $\mathrm{PC}$ at $250{ }^{\circ} \mathrm{C}$ (unfilled circles) and $290{ }^{\circ} \mathrm{C}$ (filled circles). Solid lines: approximation of the experimental data by the function $\log \Gamma_{\mathrm{o}}=\gamma_{\mathrm{o}}-\gamma_{1} v$. Curve 1: $\gamma_{0}=3.01, \gamma_{1}=1.04 \mathrm{E}-2$; curve $2: \gamma_{0}=3.81, \gamma_{1}=5.66 \mathrm{E}-2$ 154

Figure 6-45: The dimensionless parameter $\beta$ versus the filler content $v$ wt.- $\%$. Symbols: treatment of observations for virgin $\mathrm{PC}$ at $250{ }^{\circ} \mathrm{C}$ (unfilled circles) and $290{ }^{\circ} \mathrm{C}$ (filled circles). Solid lines: approximation of the experimental data by the function $\beta$ $=\beta_{\mathrm{o}}-\beta_{1}$ v. Curve 1: $\beta_{\mathrm{o}}=1.18, \beta_{1}=1.96 \mathrm{E}-2 ;$ Curve $2: \beta_{\mathrm{o}}=0.95, \beta_{1}=3.54 \mathrm{E}-2 \ldots 156$

Figure 6-46 The shear modulus G MPa versus the filler content $v$ wt $\%$. Symbols: treatment observation at $250{ }^{\circ} \mathrm{C}$ on virgin PC (unfilled circles), recycled PC (filled circles) and the mixture of $50 \mathrm{wt} \%$ virgin $\mathrm{PC}$ with $50 \mathrm{wt} \%$ recycled $\mathrm{PC}$ (asterisks). Solid lines: approximation of the experimental data by function $G=G_{0}-G_{1} v$. Curve 1: $\mathrm{G}_{\mathrm{o}}=0.45, \mathrm{G}_{1}=-8.92 \mathrm{E}-3$; curve $2: \mathrm{G}_{\mathrm{o}}=0.35, \mathrm{G}_{1}=5.8 \mathrm{E}-3$; curve $3: \mathrm{G}_{\mathrm{o}}=0.48, \mathrm{G}_{1}$ $=3.2 \mathrm{E}-3$ 157 
Figure 6-47: The dimensionless parameter $\mathrm{V}$ versus the filler content $v \mathrm{wt} \%$. Symbols: treatment observation at $250{ }^{\circ} \mathrm{C}$ on virgin PC (unfilled circles), recycled PC (filled circles) and the mixture of $50 \mathrm{wt} \%$ virgin $\mathrm{PC}$ with $50 \mathrm{wt} \%$ recycled $\mathrm{PC}$ (asterisks). Solid lines: approximation of the experimental data by function $\log V=V_{o}-V_{1} v$. Curve 1: $\mathrm{V}_{\mathrm{o}}=-0.11, \mathrm{~V}_{1}=1.73 \mathrm{E}-2$; curve $2: \mathrm{V}_{\mathrm{o}}=-0.23, \mathrm{~V}_{1}=1.02 \mathrm{E}-1$; curve $3: \mathrm{V}_{\mathrm{o}}=$ $-0.27, \mathrm{G}_{1}=7.12 \mathrm{E}-2$ 158

Figure 6-48: The attempt rate $\gamma \mathrm{s}^{-1}$ versus the filler content $v$ wt.- $\%$. Symbols: treatment of observations at $250^{\circ} \mathrm{C}$ on virgin PC (unfilled circles), recycled PC (filled circles) and the mixture of 50 wt.-\% virgin with 50 wt.-\% recycled PC (asterisks). Solid lines: approximation of the experimental data by the function $\log \gamma=\gamma_{0}-\gamma_{1} v$. Curve $1: \gamma_{o}=3.01, \gamma_{1}=1.04 \mathrm{E}-2$; curve $2: \gamma_{\mathrm{o}}=3.27, \gamma_{1}=5.68 \mathrm{E}-2$; curve $3: \gamma_{\mathrm{o}}=3.08, \gamma_{1}=$ $3.28 \mathrm{E}-2$ 160

Figure 6-49: The dimensionless parameter $\beta$ versus the filler content $v$ wt.- $\%$. Symbols: treatment of observations at $250{ }^{\circ} \mathrm{C}$ on virgin PC (unfilled circles), recycled PC (filled circles) and the mixture of 50 wt.-\% virgin with 50 wt.-\% recycled PC (asterisks). Solid lines: approximation of the experimental data by the function $\beta=$ $\beta_{\mathrm{o}}-\beta_{1}$ v. Curve 1: $\beta_{\mathrm{o}}=1.18, \beta_{1}=1.96 \mathrm{E}-2$; curve $2: \beta_{\mathrm{o}}=0.87, \beta_{1}=3.24 \mathrm{E}-2$; curve 3 : $\beta_{\mathrm{o}}=0.90, \beta_{1}=2.52 \mathrm{E}-2$ 161

Figure 6-50 Virgin polycarbonate $\tan \delta$ vs. frequency 164

Figure 6-51 Recycled polycarbonate $\tan \delta$ vs. frequency. 165

Figure $6-5250 \%$ virgin $\mathrm{PC}+50 \%$ recycled $\mathrm{PC} \tan \delta$ vs. frequency 165

Figure 6-53 Virgin polycarbonate complex viscosity vs. frequency $\left(\mathrm{T}=290{ }^{\circ} \mathrm{C}\right)$ 166 
Figure 6-54 Recycled polycarbonate complex viscosity vs. frequency $\left(\mathrm{T}=290{ }^{\circ} \mathrm{C}\right) \ldots \ldots .167$

Figure 6-55 50\% virgin polycarbonate $+50 \%$ recycled polycarbonate complex viscosity

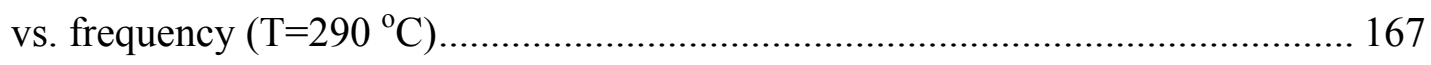

Figure 6-56 Unfilled polycarbonate complex viscosity vs. frequency $\left(\mathrm{T}=290{ }^{\circ} \mathrm{C}\right) \ldots \ldots . .168$

Figure 6-57 20\% glass fiber reinforced polycarbonate complex viscosity vs. frequency

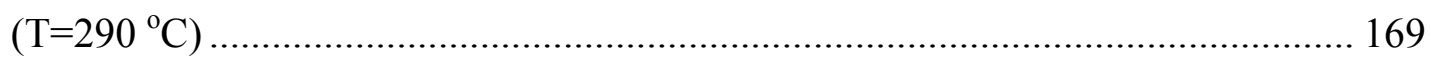

Figure 6-58 Influence of temperature on the complex viscosity of $20 \%$ glass reinforced

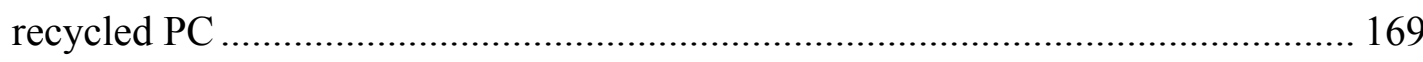




\section{List of Tables}

Table 2-1 Stress-strain properties of major thermoplastics. ..................................... 11

Table 2-2 Balance between stiffness and ductility in thermoplastics ........................... 11

Table 2-3 Flexural strength and modulus for some common thermoplastics .................. 16

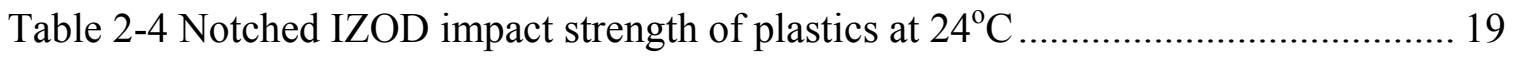

Table 2-5 Typical values for some common plastics............................................... 23

Table 2-6 Influence of molecular weight on MFI.................................................. 24

Table 2-7 Typical shear rate ranges for polymer processing..................................... 28

Table 2-8 Composition of glass used for fiber manufacture (value are in wt $\%$ ) ............. 35

Table 2-9 Typical properties of glass reinforced polycarbonate (LEXAN is manfuactured

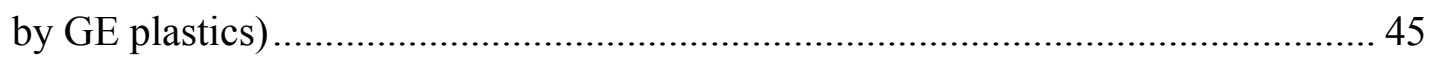

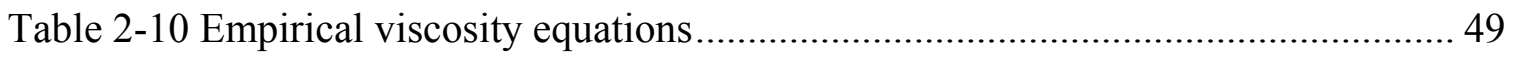

Table 3-1 Properties of recycled and virgin polycarbonate ........................................ 57

Table 4-1 Brands and sources of materials used in this research. ................................ 59

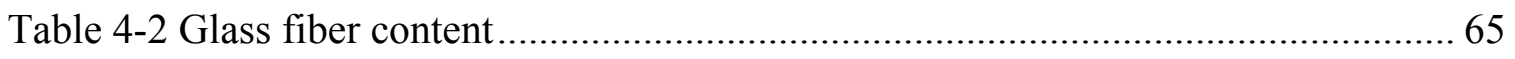

Table 5-1 Average glass fiber length for three systems............................................. 72

Table 5-2 Reinforcement efficiencies for glass reinforced polycarbonate ..................... 81

Table 5-3 15\% glass reinforced polycarbonate with impurities prediction of tensile

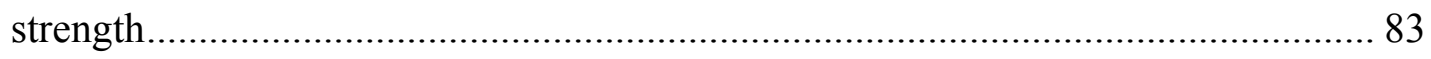

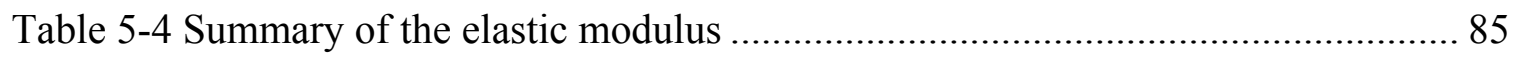

Table 5-5 Reinforcement efficiencies for glass reinforced polycarbonate ..................... 87

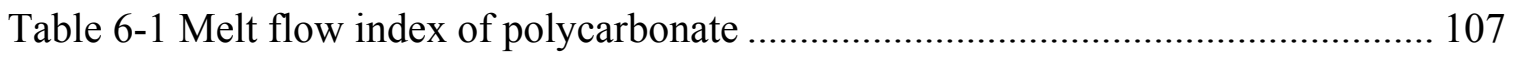


Table 6-2 Melt flow index of glass-reinforced polycarbonate................................... 107

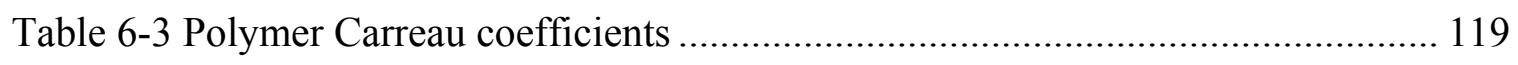




\section{List of Symbols}

$\begin{array}{ll}A & \text { Cross section area } \\ \mathrm{A}_{\mathrm{o}} & \text { Original cross section area } \\ \mathrm{ASTM} & \text { American Society for Testing and Materials } \\ \mathrm{D} & \text { Fiber diameter } \\ \mathrm{E} & \text { Elastic modulus } \\ \mathrm{E}_{\mathrm{f}} & \text { Fiber elastic modulus } \\ \mathrm{E}_{\mathrm{m}} & \text { Matrix elastic modulus } \\ \mathrm{G} & \text { Shear modulus } \\ \mathrm{G} & \text { Storage modulus } \\ \mathrm{G}^{\prime} & \text { Loss Modulus } \\ \mathrm{h} & \text { Fiber spacing } \\ \mathrm{K} & \text { Mark-Houwink-Sakurada constant } \\ \mathrm{k}_{\mathrm{B}} & \text { Boltzmann's constant } \\ \mathrm{L} & \text { Fiber length } \\ \mathrm{l}_{\mathrm{o}} & \text { Original gage length } \\ \mathrm{MFI} & \text { Melt flow index } \\ \mathrm{MFR} & \text { Melt flow rate } \\ \mathrm{M}_{\mathrm{w}} & \text { Molecular weight } \\ \mathrm{n} & \text { Power law index } \\ \mathrm{N} & \text { number of active strands per unit mass } \\ \mathrm{ROM} & \text { Rule of Mixtures } \\ \mathrm{T} & \text { Temperature } \\ \mathrm{T}_{\mathrm{g}} & \text { Glass transition temperature } \\ \mathrm{T}_{\mathrm{m}} & \text { Melting temperature } \\ \mathrm{v} & \text { Volume fraction } \\ \mathrm{v} & \text { Activation energy } \\ & \end{array}$

\section{Greek}

$\begin{array}{ll}\alpha & \text { Mark-Houwink-Sakurada constants } \\ \beta & \text { Constant } \\ \delta & \text { Tan Delta } \\ \varepsilon & \text { Strain } \\ \dot{\gamma} & \text { Shear rate } \\ \Gamma & \text { Rate of detachment } \\ \eta & \text { Viscosity }\end{array}$




$\begin{array}{ll}\eta_{\mathrm{o}} & \text { Zero shear viscosity } \\ {\left[\eta^{*}\right.} & \text { Intrinsic viscosity } \\ \eta^{*} & \text { Complex Viscosity } \\ \varphi & \text { Rate of reformation for dangling chains } \\ \lambda & \text { Characteristic relaxation time in seconds } \\ \nu & \text { Volume fraction } \\ \rho & \text { Density } \\ \sigma & \text { Stress } \\ \sigma_{\mathrm{m}} & \text { Tensile strength of the matrix } \\ \sigma_{\mathrm{f}} & \text { Tensile strength of the fiber } \\ \sigma_{\mathrm{c}} & \text { Tensile strength of the composite } \\ \tau & \text { Shear stress } \\ \omega & \text { Frequency }\end{array}$




\section{Introduction}

The rapid growth in production of plastics has led to a greater awareness of the need to recycle plastic materials. Firms specializing in recycling have sprung up around the world, and in 1994, there were an estimated 1000 plastics recycling companies in Europe. In the United States at this time there were approximately 400 of these companies, while Japan lagged behind with a mere handful ${ }^{1}$. Despite the vast amounts of plastic goods that were produced at this time, only a small fraction of the discarded plastic was recycled: approximately 4.5 percent in the United States and 7.5 percent in Europe $^{1}$. In 1980, in the United States, approximately 20,000 tons of post-consumer plastics were recycled. By 1999, this number had leapt to about 1,400,000 tons ${ }^{2}$. Production of plastics in the United States in 1999 had reached 24,000,000 tons of plastics. There is, however, a wide variation in the reliability of sources that reported recycling rates. In 1994, the American Plastics Council (APC) reported a recycling rate of about 21 percent for plastic bottles and about 17 percent for plastic containers. This showed a significant increase in recycling over the 4 percent rate reported five years earlier $^{3}$. The APC estimated the total tonnage of plastics recycled in 1994 as 1 billion pounds ${ }^{4}$. For certain types of bottles, the rate was much higher, at almost 49 percent for PET soft-drink bottles and nearly 26 percent for natural HDPE bottles ${ }^{4}$. An estimated 15,000 communities in the United States are currently reported to be actively involved in collecting plastics for recycling 5 .

In the 1970's, high oil prices made the economic viability of recovering process scrap through regrinding an appealing alternative to discarding the material. This method is still used to recover uncontaminated process scrap (clean, single-resin material). 
Strictly speaking, however, the regrinding method is not considered to be recycling, but is simply a part of the normal cycle of material use. Although it is known that large amounts of the contaminated scrap are still discarded, there is little reliable quantitative information on the disposal of this material. It is known, however, that the recognition of the economic worth of the recycled materials has led to a rise in the recovery rate of preconsumer plastics. New technologies and the development of conversion facilities also make the process of recycling more economically viable.

Since the production and recycling of consumer electronics has been increasing dramatically in the past years, there is a recent need to find ways to recycle polycarbonate. One of the reasons that this study's focus in on polycarbonate is because it is a major thermoplastic that is used in making these electronics. Polycarbonate is also considered expensive when compared with other types of thermoplastics, such as PET and HDPE. The need to recycle polycarbonate is important from both the environmental and economic point view. There is a certain amount of recycling of polycarbonate from products such as automobile fenders, and common consumer goods such as computers, telephones, plastic water bottles and compact discs. Several years ago General Electric established a policy of purchasing polycarbonate recycled from 5-gallon water bottles. In 1994, there were signs of a definite trend in recycling when manufacturers at the National Plastics Exposition (NPE) in Chicago introduced several new polycarbonate resins that had been produced from recycled materials ${ }^{6}$. Most had 25 percent pre or post-consumer recycled content. The properties of the material were closely related to those of virgin materials, although they had $50 \%$ less impact strength and tensile elongation was reduced by $33 \%$. In addition, these resins were not recommended for use 
where there is a need for strict color tolerance. The economic viability of the recycled material is, however, undeniable, with the recycled resin costing half that of the virgin material.

The first factory dedicated to recycling polycarbonate from Compact Discs was constructed in 1995 in Dormagen, Germany. The polycarbonate from aluminum coatings, protective layers, and printing, is separated, blended and sold for various uses ${ }^{7}$. Polycarbonate reclaimed from water-cooler bottles is being recycled into headlamp housings for Ford automobiles, and Chrysler now uses PC recyclate for instrument-panel covers ${ }^{8}$.

After the material has been recycled and separated, the properties of the recycled polymer differ greatly from the properties of the virgin material. The aim of this research is to show how the properties of the recycled polymer can be improved through reinforcing the recycled polycarbonate with glass-fibers. 


\section{Literature Review}

\subsection{Polycarbonates}

There are two major classes of Polycarbonates: aliphatic and aromatic ${ }^{9}$. Aliphatic polycarbonates have no significant commercial use as thermoplastics. Most of the aliphatic polycarbonates are semi-crystalline materials with low melting points, normally less than $120{ }^{0} \mathrm{C}$. Aliphatic polycarbonates are used as plasticizers ${ }^{\mathbf{1 0}}$, as comonomers ${ }^{\mathbf{1 1}}$, and in copolymers such as polyurethane and blends with bisphenol A polycarbonates.

The most common aromatic polycarbonate is that derived from bisphenol A. Poly(bisphenol A carbonate), also known as polycarbonate (PC), is an amorphous polymer that possesses high heat resistance, and it is tough and transparent ${ }^{12}$. The use of PC ranges from small consumer products such as tumblers to large-part automotive and construction applications.

With its broad product applicability, the production of PC has grown tremendously since its initial commercialization in 1958. In 1991 the annual worldwide capacity of PC was 800,000 metric tons; it is now estimated to be over 1.1 million tons. This figure is expected to continue to grow as more applications are being developed. Currently, over $70 \%$ of the PC is manufactured by three major producers: by General Electric under the trade name Lexan; by Bayer (Bayer AG) under the trade name of Makrolon, and by the Dow Chemical Company under the trade name CALIBRE. Currently, $44 \%$ of the PC is produced in the United States, $26 \%$ in the Asia/Pacific region, and 30\% in Europe. Other commercial uses of PC are in polymer blends such as PC/ABS blends. 


\subsubsection{Synthesis of polycarbonate}

Poly(bisphenol A carbonate), or PC, is a condensation product of bisphenol A, a carbonate precursor such as phosgene or diphenyl carbonate, and a monophenol chain terminator such as phenol or t-butyl phenol ${ }^{13}$. The structure of a linear PC is as shown:

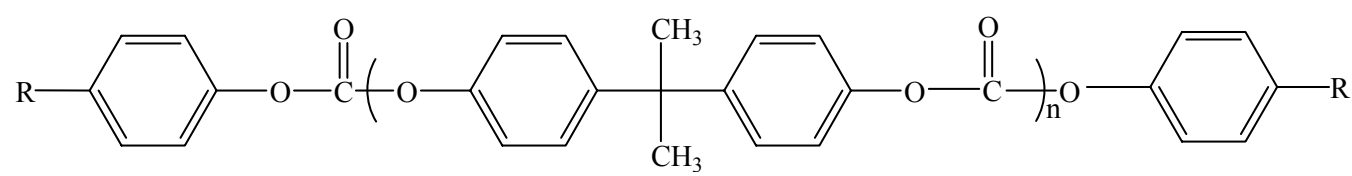

Figure 2-1 Bisphenol A Polycarbonate (PC)

The most commercially successful method for manufacturing Polycarbonate is interfacial polymerization. An interfacial process involves two-phase separated liquid systems wherein reaction occurs at the interface of the two phases. This has been the most widely practiced method and is used for over $95 \%$ of the worldwide commercial production of bisphenol A PC. In this method an aqueous solution of bisphenol A and sodium hydroxide is reacted with phosgene to form oligomers, which then transfer from the aqueous phase to the organic phase, usually a chlorinated solvent such as methylene chloride.

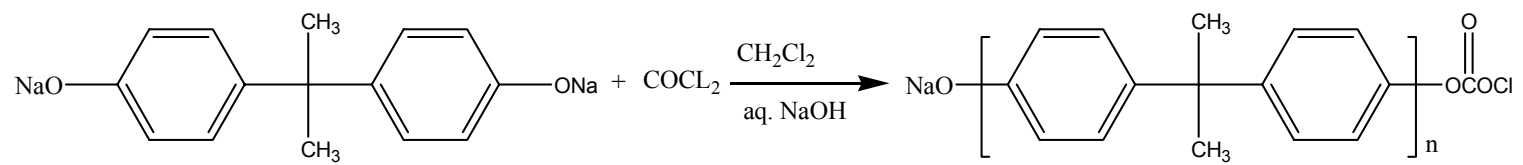

The molecular weight of the oligomers continues to grow in the organic phase at the aqueous organic interface. The ratio of the chloroformate end group to the hydroxy 
end groups in the oligomers depends on $\mathrm{pH}$, phosgene addition time, and amount of phosgene added $^{\mathbf{1 4}}$. This ratio can affect the molecular weight control of the final polymer. An excess of chloroformate is usually desirable to obtain a high molecular weight PC. The oligomers are further polymerized to form the high molecular weight polymer by the addition of a catalyst such as triethylamine.

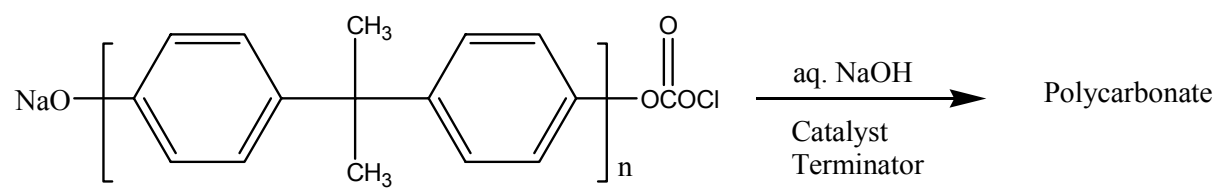

A monophenolic terminator is added to control the molecular weight. The terminator type can slightly influence the impact properties of the polymer ${ }^{15}$.

Both batch and continuous processes are practiced industrially. This reaction is exothermic and requires removal of heat. The phosgenation reaction is fast and can be completed using static mixers in a tubular reactor. Usually phosgene addition/heat removal controls the phosgenation time in the batch reactors.

After polymerization is complete, the aqueous portion is normally removed using a centrifugal separator. If desired, the polymer is then washed with a dilute base to remove the excess monomers in the organic phase, followed by contact with acid to neutralize the base and extract the catalyst. The acidified polymer is then washed with water to remove the salt.

Some of the methods used to remove the solvent from the polymer are (1) steam flashing of methylene chloride, (2) anti-solvent precipitation, and (3) contacting with hot water with mechanical kneading. The porosity and the size of the particles produced 
depends on the method used. Sometimes a finishing step, such as countercurrent stripping, is required to achieve very low levels of residual solvents. A drying step to remove the water is also needed when steam or hot water is used for evaporating the solvent.

\subsection{Properties of polycarbonates}

\subsubsection{Molecular weights and molecular weight distributions}

Since many polymer properties are dependent on molecular weight, special attention is paid to the characterization of Polycarbonate molecular weight. The molecular weights of PC can be discerned using several methods. A common method reported in the literature is known as dilute solution viscosity. This method measures the viscosity average molecular weight utilizing the Mark-Houwink-Sakurada relationship:

$$
[\eta]=\mathrm{KM}_{\mathrm{v}}^{\alpha}
$$

where, $\mathrm{K}$ and $\alpha$ are constants that depend on the solvent used and the test temperature and $[\eta]$ is intrinsic viscosity as a function of the volume average molecular weight. In most cases, methylene chloride is used and the test temperature is $25{ }^{0} \mathrm{C}$. In that case, the constant $\mathrm{K}$ is $1.19 \times 10^{-4}$ and $\alpha$ is $0.8^{\mathbf{1 6}}$ for [ $\left.\eta\right]$ in $\mathrm{dl} / \mathrm{g}$. Other common solvents used are chloroform $^{17}$, tetrahydrofuran, dioxane, and ethylene chloride ${ }^{\mathbf{1 8}}$.

Another common method for determining the molecular weight of PC is gel permeation chromatography with an ultraviolet (UV) detector (GPC-UV). The GPC results 
give the complete molecular weight distribution from which the number average, weight average, and z-averag molecular weights can be determined.

Depending on the method of synthesis, molecular weight averages of PC can be obtained in a range from 13,000 to about $200,000 \mathrm{~g} / \mathrm{mol}^{7}$. The most useful range of PC molecular weight averages is $\mathrm{Mw} 24,000-80,000 \mathrm{~g} / \mathrm{mol}$. The lower molecular weight range can be extended down to about $17,000 \mathrm{~g} / \mathrm{mol}$ and is used for optical disk applications. At the upper range, PCs with molecular weights greater than $38,000 \mathrm{~g} / \mathrm{mol}$ have a high melt viscosity and are very difficult to melt-fabricate in a conventional manner. Applications of PC with molecular weights above 70,000 g/mol include cast sheet and films ${ }^{19}$, and blends with lower molecular weight PCs for improved toughness ${ }^{20}$.

\subsubsection{Stress-strain behavior}

Of the various measurements of mechanical properties made on materials, stressstrain behavior in tension is one of the most common. For many polymers this is specified by the ASTM D 638, Standard Method of Test for Tensile Properties of Plastics. In this test a dumbell-shaped sample is clamped into the jaws of a testing machine, and the load that is required to elongate it at a given rate is recorded. Ideally, the elongation is measured over a portion of the narrowed section of the sample (termed the gage length) by means of an extensometer ${ }^{21}$.

This load versus the elongation curve can be converted to a more general stressstrain curve by dividing the ordinate values by the original cross-sectional area of the narrow region of the sample $\left(\mathrm{A}_{0}\right)$ and dividing the abscissa values by the original gage length $\left(1_{0}\right)$. Since $A_{o}$ and $l_{o}$ are constants, the stress-strain curve has the same general 
shape as the load-elongation curve.

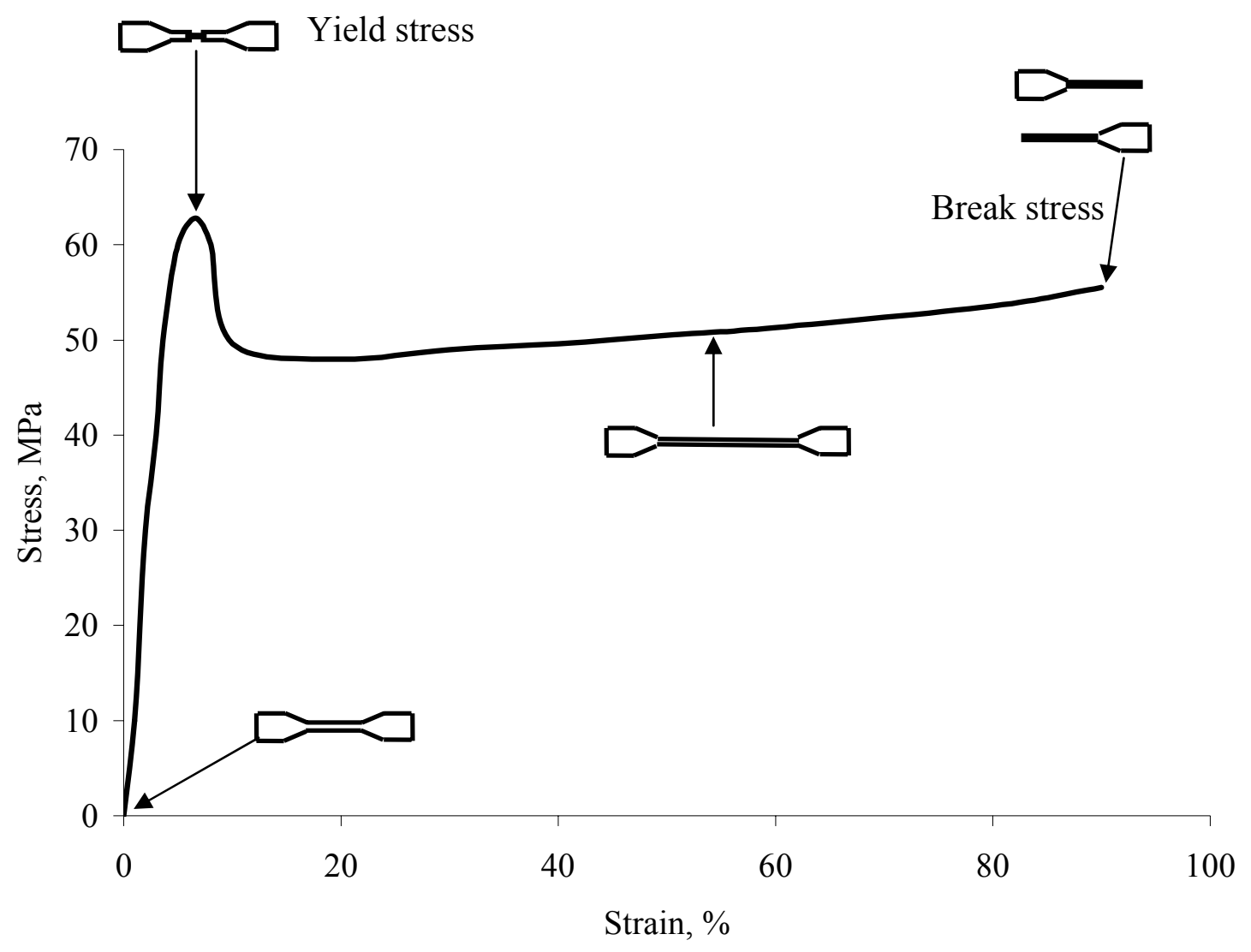

Figure 2-2 Typical stress-strain curve for polycarbonate

A nominal stress-strain curve contains much useful information about a material (see Figure 2-2). This includes:

Elastic modulus: This is the slope of the initial (approximately linear) portion of the curve. It is given by:

$$
\mathrm{E}=\frac{\mathrm{P}_{1} / \mathrm{A}_{\mathrm{o}}}{\Delta \mathrm{l}_{1} / \mathrm{1}_{\mathrm{o}}}
$$


Yield stress: This is defined as the first stress value for which $\mathrm{d} \sigma / \mathrm{d} \varepsilon=0$.

Ultimate stress: This is the maximum stress value.

Toughness: This is the total area under the stress-strain curve. The units for toughness are $\mathrm{J} / \mathrm{m}^{3}$, or energy per unit volume of the original material.

When polymers are tested in tension according to ASTM D 638, three general types of behavior are frequently observed. Figure 2-3 illustrates the great variation in stress-strain behavior of polymers, as measured at a constant rate of strain. The first graph (A) is for hard, brittle materials. The second graph (B) is typical of hard, ductile polymers. The top curve in the ductile polymer graph is for material that shows uniform extension. The lower curve in this graph has a yield point. Curves of the third graph $(\mathrm{C})$ are typical of elastomeric materials. Table 2-1 compares the stress-strain properties of polycarbonate with other commonly encountered thermoplastics. It is found that polycarbonate has both high strength and high ductility, making it desirable for applications that require these two properties. Nylon and PET have high strength but low ductility, polyethylene and polypropylene have high ductility but low strength. Comparing polycarbonate with the other four thermoplastics, it is found that it is the one that combines both strength and ductility. The ideal combination of characteristics would be high modulus and high strength with ductility. The inherent nature of plastics is such, however, that high modulus tends to be associated with low ductility and any steps taken to strengthen one characteristic cause the other to deteriorate. The major effects are summarized in Table 2-2. 
Table 2-1 Stress-strain properties of major thermoplastics ${ }^{22}$.

\begin{tabular}{lccc}
\hline \hline Polymer & $\begin{array}{c}\text { Stress at break } \\
\text { psi }\end{array}$ & $\begin{array}{c}\text { Tensile modulus } \\
\text { psi }\end{array}$ & $\begin{array}{c}\text { Elongation at break } \\
\%\end{array}$ \\
\hline Polycarbonate & 9500 & 345000 & 120 \\
Nylon & 13700 & 230000 & 15 \\
PET & 7000 & 400000 & 30 \\
Polyethylene & 3200 & 155000 & 300 \\
Polypropylene & 4500 & 165000 & 100 \\
\hline \hline
\end{tabular}

Table 2-2 Balance between stiffness and ductility in thermoplastics

\begin{tabular}{lll}
\hline \hline & & Effect on \\
& Modulus & Ductility \\
Increase straining rate & Increase & Decrease \\
Incorporation of plasticizer & Decrease & Increase \\
Incorporation of rubbery phase & Decrease & Increase \\
Incorporation of glass fibers & & Decrease \\
Incorporation of particulate fillers & Increase & Decrease \\
Reduce temperature & Increase & Decrease \\
\hline \hline
\end{tabular}



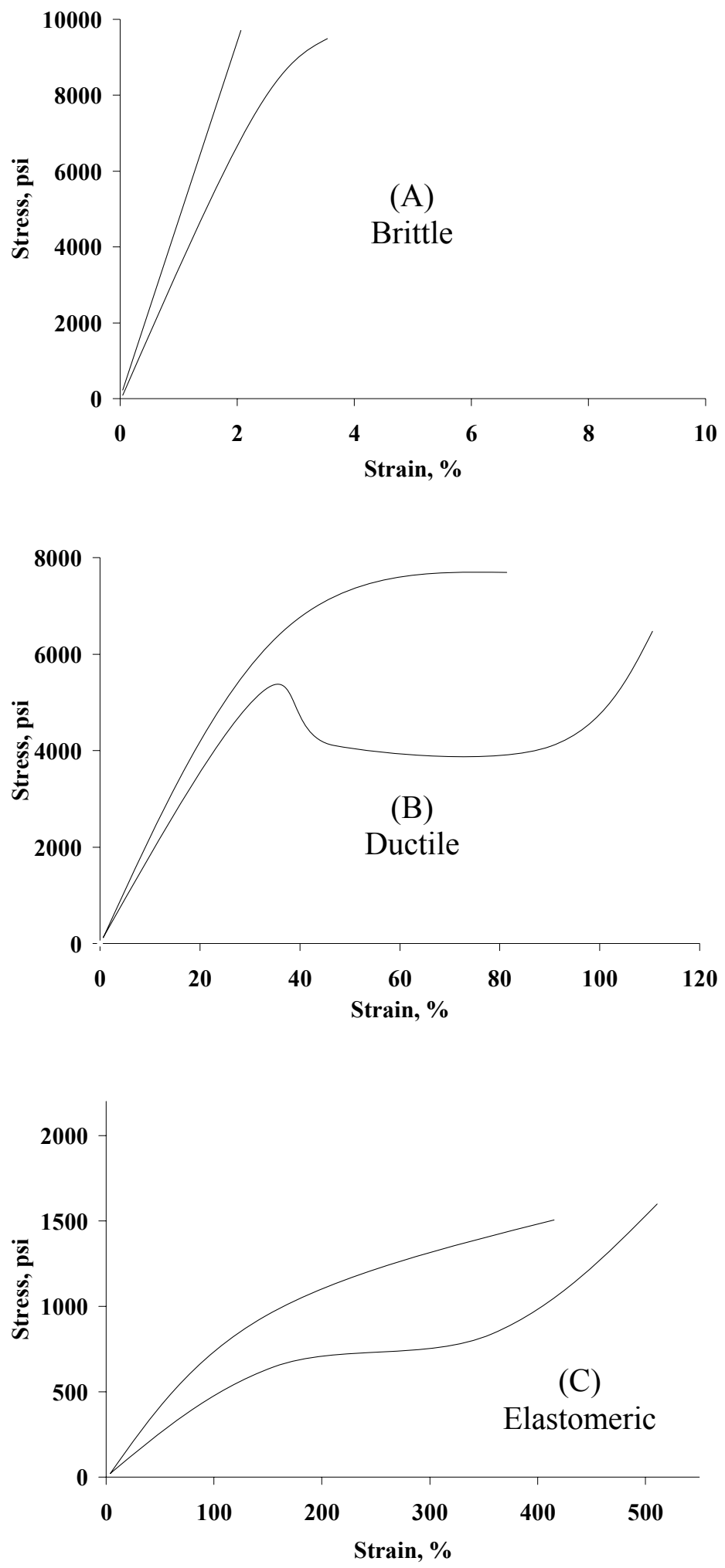

Figure 2-3 General types of stress-strain curves 
Many of the characteristics of polymers are influential in determining tensile behavior of a given polymer. These characteristics include:

- The structure of the repeating unit.

- The flexibility of the molecules.

- The molecular weight.

- The presence or absence of crosslinks.

- Crystallinity and the orientation of molecules.

- Temperature and its relationship to $\mathrm{T}_{\mathrm{g}}$ and $\mathrm{T}_{\mathrm{m}}$.

\subsubsection{Flexural properties}

Flexural properties are measured to obtain a measure of stiffness or rigidity. Flexural strength is the strength determined from the load and the test piece dimensions in a flexural test. Flexural strength is also known as "breaking strength" and is usually measured by applying stress at the center of a rectangular bar, which is supported at two other points (see Figure 2-4). That is, three-point loading is applied in a bending test. During the test, the force applied and the resulting deflections are measured. The test is usually performed on a universal testing machine (as used for tensile strength tests).

Bending the specimens in a three-point-bending jig (on which the span $\mathrm{x}$ distance between the two outer supports is set 15 to 17 times the thickness) gives a load/deflection curve. From this curve flexural strength and modulus are determined.

If the specimen is brittle and breaks at very low strain (less than 0.05), then the breaking or flexural strength is calculated from equation $2-3^{23}$ : 
Load (F)

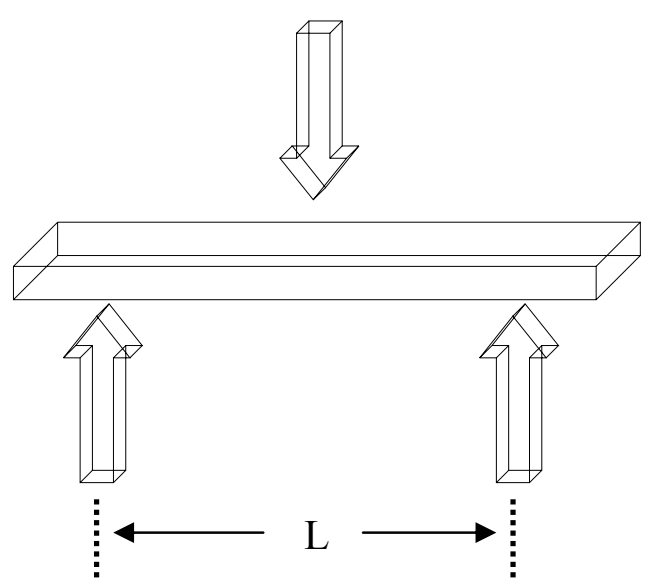

Figure 2-4 Schematic of flexural test

$$
\sigma_{\mathrm{B}}=\frac{3 \mathrm{~F}_{\mathrm{B}} \mathrm{L}}{2 \mathrm{bh}^{2}}
$$

Where $\sigma_{B}$ is the flexural strength, $F_{B}$ is the load at break, $L$ is the span width, $b$, and $\mathrm{h}$ are width and thickness of the specimen, respectively. If the sample does not break but simply bends then the stress measured when the sample deflection reaches 1.5 times the sample thickness is used. The breaking strain is often given the symbol $\gamma$ and equals $\frac{6 \mathrm{Dh}}{\mathrm{L}}$ where $\mathrm{D}$ is the deflection at mid-span. The elastic modulus in flexure, E , can be calculated from

$$
\mathrm{E}=\frac{\mathrm{L}}{4 \mathrm{bh}^{3}} \frac{\mathrm{F}}{\mathrm{Y}}
$$

where $\mathrm{F} / \mathrm{Y}$ is the slope of the initial linear load-deflection curve. Table 2-3 lists some flexural strength and modulus for some commercial thermoplastics. Polycarbonate has high flexural strength and flexural modulus, making it attractive in applications where 
bending strength is needed.

Table 2-3 Flexural strength and modulus for some common thermoplastics

\begin{tabular}{lcc}
\hline \hline Polymer & Flexural strength, psi & Flexural modulus, psi \\
\hline ABS & 10800 & 380000 \\
Polycarbonate & 14000 & 340000 \\
PET & 16000 & 460000 \\
HDPE & 2000 & 100000 \\
Polypropylene & 6000 & 200000 \\
Nylon & 15500 & 420000 \\
\hline \hline
\end{tabular}

\subsubsection{Impact strength}

Impact tests are high-speed fracture tests that measure the energy required to break a specimen. In the Izod and Charpy impact tests a pendulum with a hammerlike weight strikes a specimen, and the energy required to break the specimen is determined from the loss in kinetic energy of the weight ${ }^{24}$. In the falling ball or falling dart impact tests the amount of energy required to break a sheet or plate of the material is determined from the weight of the ball and the height from which it was dropped ${ }^{25}$. The Gardner impact test is one example of a falling dart type of impact test. Generally, Izod and Gardner tests do not correlate well. Still another type of impact test measures the area under the curve obtained in a high-speed tensile stress-strain test ${ }^{26}$. 
In addition to the fact that there is often little uniformity between the results of the various impact tests, the polymers may also not be ranked consistently for different tests. As well, a given type of impact test does not give a value that is a material constant. The geometry of the specimen is important, since the impact strength depends on sample size, even if an attempt is made to normalize the values to a constant specimen size. Thin specimens tend to give higher impact strengths than do thick ones. The disagreement between different kinds of tests indicates that impact tests are controlled by at least two or more basic physical properties. Two factors governing impact behavior are (1) the energy needed to initiate a crack and (2) the energy required to propagate a crack.

The units used to express impact strength can be confusing. In high-speed tensile tests and similar tests on un-notched specimens, the impact strength is defined in terms of the area under the stress-strain curve or as energy to break, with a unit such as $\mathrm{ft}-\mathrm{lb} / \mathrm{in}^{3}$. For notched IZOD and Charpy tests, impact strength is defined in terms of energy per length of notch with a unit such as ft-lb/in.

In the IZOD test a small, notched cantilever beam is broken with a pendulum hammer (Figure 2-5 and Figure 2-6) ${ }^{27}$. The purpose of the notch is to provide crack initiation. The notch in the sample is done by using a notcher (Figure 2-7). The energy required to break the sample is determined from the height reached by the pendulum after the sample is broken. 


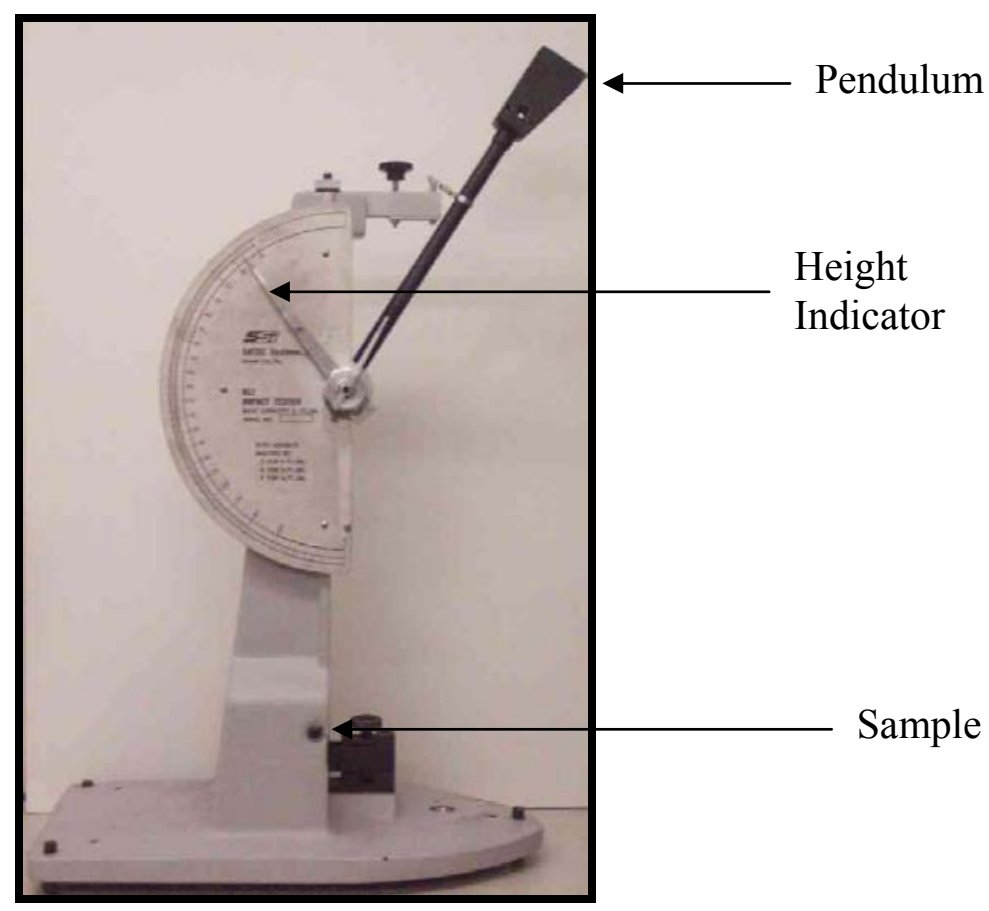

Figure 2-5 IZOD impact tester (from ASTM D-256)

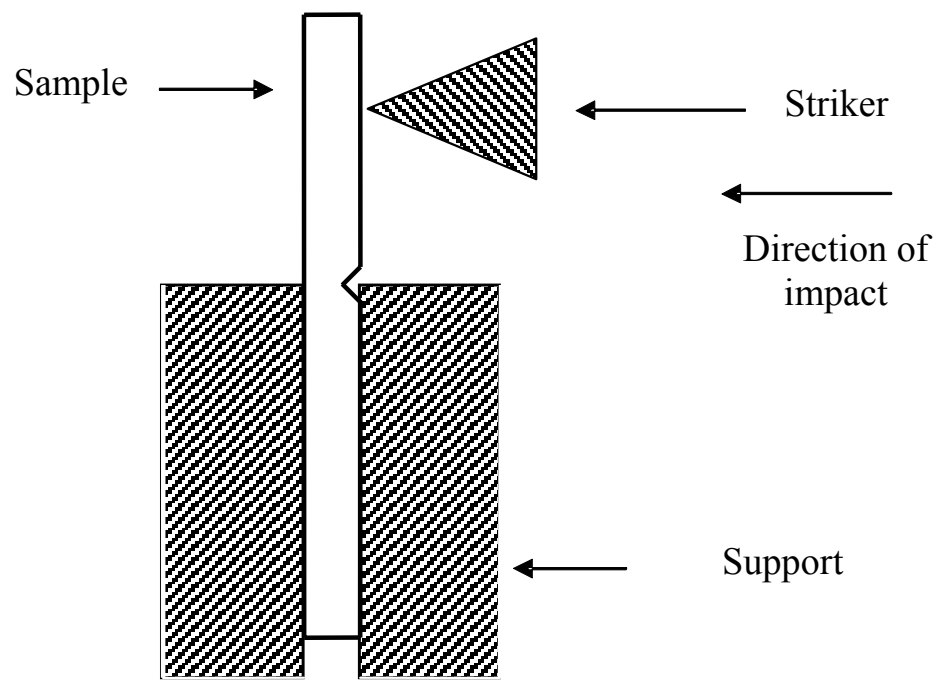

Figure 2-6 Mounting of IZOD impact specimen 
Table 2-4 lists the typical range of notched IZOD impact strengths for some common plastics. The values for a given polymer can vary over a wide range because impact strength is strongly sensitive to parameters other than those derived directly from the structure of the repeating unit. These include crystallite size, molecular weight, temperature (particularly in relation to glass transition temperature, $\mathrm{T}_{\mathrm{g}}$ ), molecular orientation, and the presence of plasticizers, fillers, and reinforcing agents. From Table 24 , it is seen that the major advantage of polycarbonate over other thermoplastics is its high impact strength, making it desirable for applications where high impact strength is needed. For example, if a device like a cellular phone is made from Nylon and is dropped the chances of breakage are much higher than if the cellular phone is made from polycarbonate.

Table 2-4 Notched IZOD impact strength of plastics at $24^{\circ} \mathrm{C}$

Plastic Impact strength (ft-lb/in)

\begin{tabular}{ll}
\hline Polycarbonate $12-18$
\end{tabular}

Nylon 66

Polyethylene (high density)

Polypropylene

ABS $1.0-10$

Polystyrene

$0.25-0.40$ 


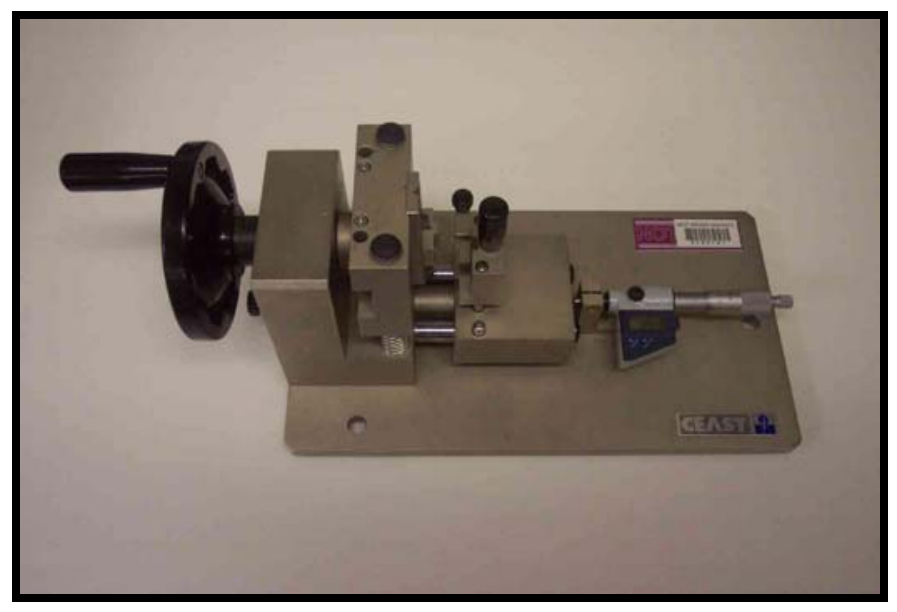

Figure 2-7 Notcher used to notches according to the ASTM standard

\subsubsection{Shore hardness}

The hardness measurement has wide applications in the characterization of the mechanical and physical properties of materials (for example the resistance to scratching). Hardness is generally defined as the resistance of a material to local surface deformation. In an indentation test, a softer material is indented by a rigid indenter of specified tip geometry (conical, spherical, pyramid etc.) and hardness is usually computed as the ratio of indentation load to the projected area of contact between the indenter and the material in the plane of deforming surface. The area of contact may be measured actually, or indirectly, from the image of the residual indent on the softer surface after the indenter is removed. In this case the hardness value is controlled by the plasticity of the material. The actual choice of the technique used for hardness measurement depends to a great extent upon the type of the material tested and the kind of information sought from the test. For elastomeric materials such as rubbers, the rebound hardness test is most commonly used. 
Figure 2-8 shows a common procedure used in the hardness measurement. Different standards have been formulated for the measurement of normal indentation hardness. They are based on different geometrical shapes of the indenter. The most commonly used are (a) Brinell (sphere), (b) Vickers (pyramid), (c) Rockwell (cone and sphere).

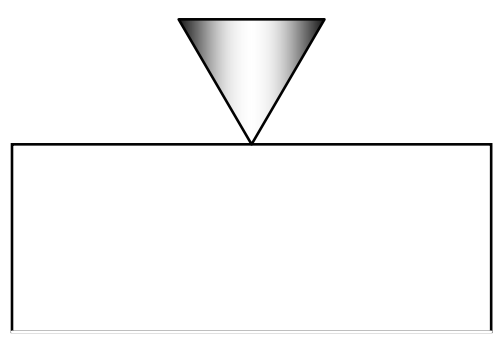

(a) Initial contact

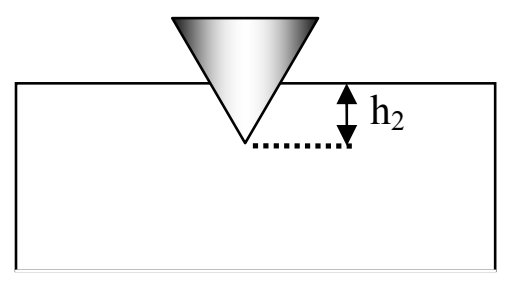

(c) After the initial unloading

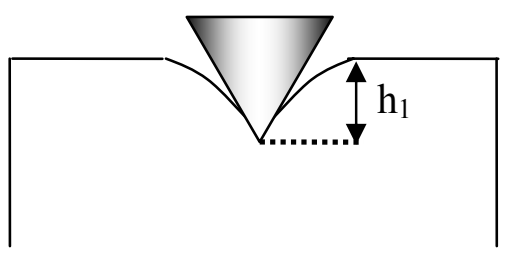

(b) At the maximum depth or maximum load

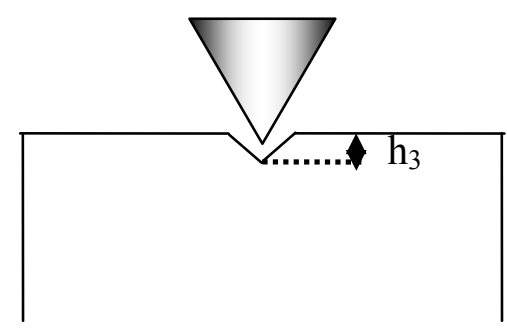

(d) After complete unloading

Figure 2-8 Schematic representation of the procedure of normal harness testing $\left(\mathrm{h}_{1}>\mathrm{h}_{2}>\mathrm{h}_{3}\right)$ 
The Shore Hardness is a measure of the hardness or softness of a material, as assessed by the resistance (on a scale of 0 to 100) experienced by an indenter. High resistance, such as that produced by a sheet of glass, would be indicated as 100, with zero resistance being described as 0 . Two different indenters are used: Type A for soft materials and Type D for hard materials. This means that there are two scales of Shore Hardness, Shore A and Shore D.

Type A measurements are made on soft materials using a truncated $35^{\circ}$ cone with a blunt tip of $0.79 \mathrm{~mm}$ diameter as an indenter. Type $\mathrm{D}$ measurements are made on harder materials with a $30^{\circ}$ steel cone rounded to $0.1 \mathrm{~mm}$ radius tip as an indenter. The depth of penetration is inversely indicated in thousandths of an inch. The harder the surface the higher the reading.

The specimen must be conditioned prior to testing and it has to be sufficiently flat over a radius of at least $6 \mathrm{~mm}$ from the indenter point to allow contact with the foot of the instrument. It must also have a thickness of at least $6 \mathrm{~mm}$ unless it is known that identical results can be obtained with a thinner specimen. Measurements are made at several points over the surface simply by pressing the instrument against the surface of the specimen. Readings should be taken after a standard period of time; the ASTM D2240 suggests one second ${ }^{28}$. Table 2-5 lists the typical hardness value for some common plastics. 
Table 2-5 Typical values for some common plastics

\begin{tabular}{lc}
\hline \hline Plastic & Shore Hardness D \\
\hline Polycarbonate & 60 \\
Nylon & 80 \\
High-Density polyethylene & 70 \\
Polypropylene & 74 \\
Polystyrene & 74 \\
\hline \hline
\end{tabular}

\subsubsection{Optical properties}

PC has a nominal refractive index of 1.538 compared to that of 1.52 for glass. The refractive index varies with the wavelength of the incoming light beam. At a wavelength of greater than $600 \mathrm{~nm}$, the refractive index is in the range of 1.58 and increases to 1.61 at a wavelength of $400 \mathrm{~nm}^{29}$. This optical property coupled with the toughness of polycarbonate are unique engineering thermoplastics qualities that are ideal for use in precision optical applications.

\subsubsection{Rheology}

The melting point, also known as the melting temperature, is the temperature at which heat renders a solid material into a liquid. It is the temperature at which the solid and liquid phases of a material are in equilibrium at a specified pressure (usually atmospheric). Unlike materials which have a low molecular weight, most polymers do not normally have a sharp melting point. Even a semi-crystalline thermoplastic has a 
melting range covering as much as $20{ }^{\circ} \mathrm{C}$. An amorphous thermoplastic does not exhibit a melting point, but on heating, will soften as the temperature increases, until it becomes a high viscosity melt. Polycarbonate has a $\mathrm{T}_{\mathrm{g}}$ (Temperature at which a plastic changes from a rigid state to a softened state) around $150{ }^{\circ} \mathrm{C}$. Polycarbonate is typically processed at a temperature of about $300{ }^{\circ} \mathrm{C}$.

Polycarbonates are commonly specified in terms of melt flow rate (MFR). The melt flow rate is determined using a melt indexer (Figure 2-9) as described in ASTM D1238. The test conditions are a $1.2-\mathrm{kg}$ load at $300{ }^{\circ} \mathrm{C}$. The MFR, as measured for linear PC, can be correlated to the molecular weights via the zero shear viscosity ${ }^{30}$. Table 2-6 shows the influence if molecular weight on the melt flow index (MFI) ${ }^{31}$.

Table 2-6 Influence of molecular weight on MFI

\begin{tabular}{lcc}
\hline Polycarbonate & $\mathbf{M}_{\mathbf{w}}, \mathbf{G P C}$ & MFI (g/10 min) \\
\hline PC63 & 72600 & 3.2 \\
PC58 & 66100 & 4.9 \\
PC55 & 62000 & 6.5 \\
PC54 & 60600 & 7.4 \\
PC51 & 57000 & 9.2 \\
PC49 & 54500 & 11.2 \\
PC45 & 49800 & 16.2 \\
PC43 & 46900 & 20.9 \\
PC35 & 44891 & 27 \\
\hline \hline
\end{tabular}




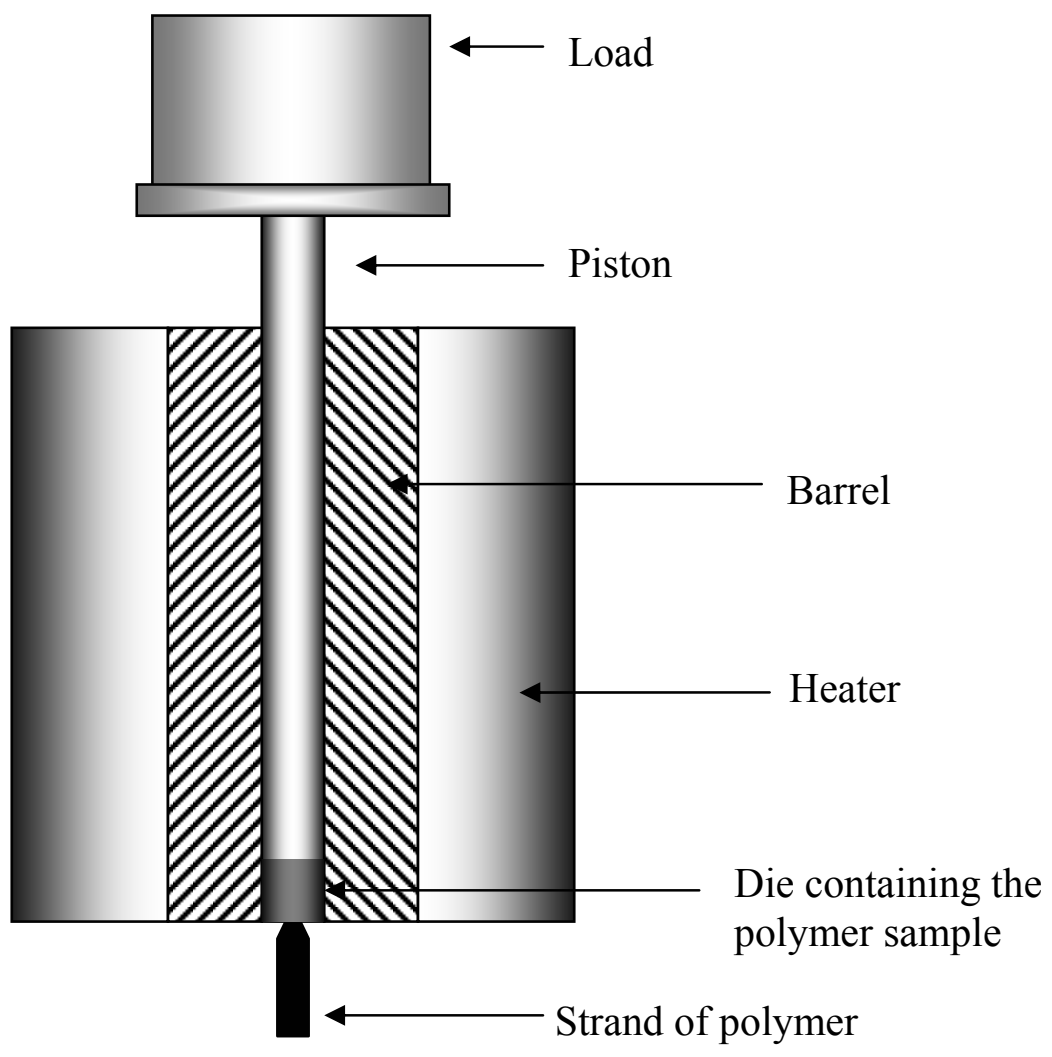

Figure 2-9 Schematic diagram of the melt flow index apparatus showing cross section view of the important parts

The melt flow behavior of PC is quite different from other polymers. It possesses a wide range of shear rates where the shear viscosity is Newtonian. At high shear rates, the shear viscosity is pseudoplastic. The critical shear rate at which the viscosity changes from Newtonian to pseudoplastic behavior decreases with increasing $\mathrm{M}_{\mathrm{w}}$. Figure 2-10 shows a typical characteristic flow curve of PC resin. In this figure, the material is tested at 270,285 , and $300{ }^{0} \mathrm{C}$. The shear viscosity as a function of shear rate and temperature can be described using the modified Cross model with an Arrhenius or the WLF 
temperature dependence expressions. For the WLF form, the modified Cross model can be expressed as:

$$
\eta(\gamma, \mathrm{T})=\frac{1.5 \rho \mathrm{T} \lambda}{1+(\lambda \gamma)^{0.8}}
$$

where $\rho$ is the melt density in $\mathrm{kg} / \mathrm{m}^{3}, \mathrm{~T}$ is the absolute temperature in Kelvin, $\lambda$ is the characteristic relaxation time in seconds, and $\gamma$ is the shear rate.

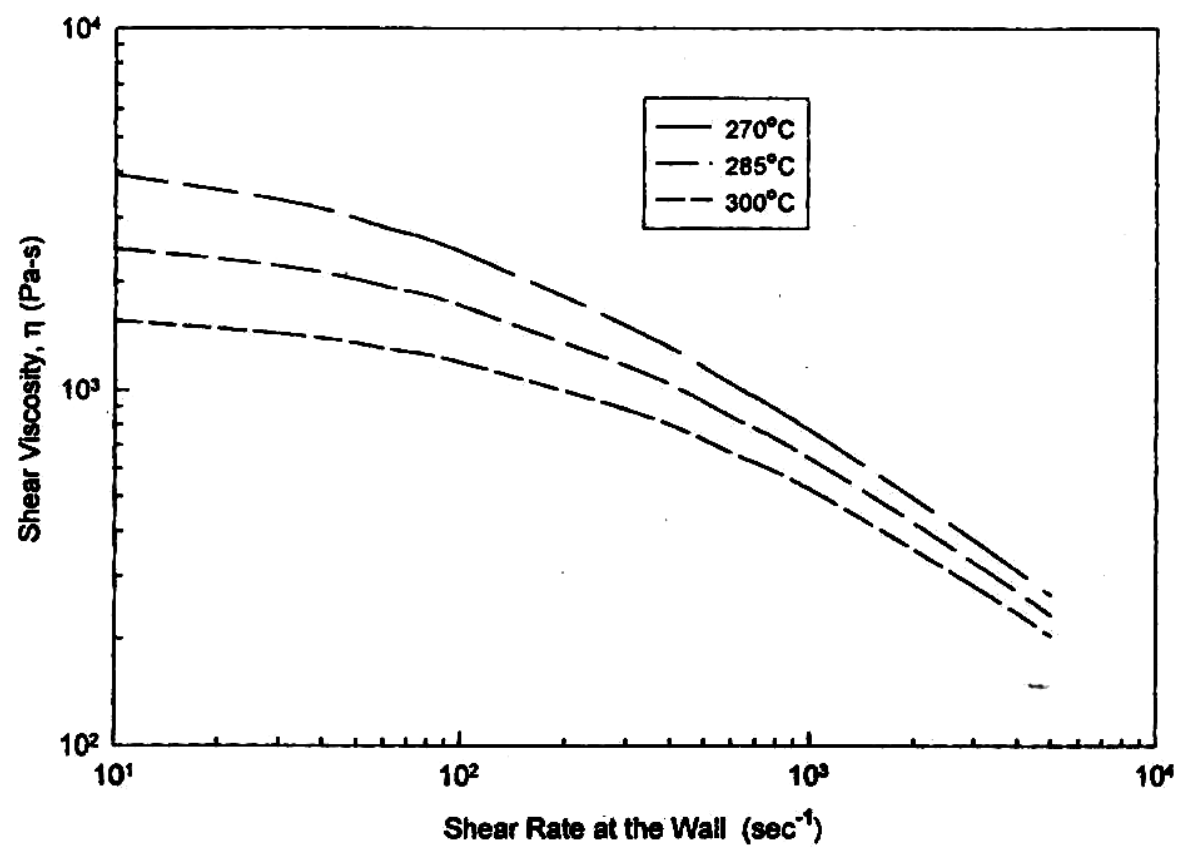

Figure 2-10 Characteristic flow curves of $37,000 \mathrm{~g} / \mathrm{mol}$ polycarbonate resin at various temperatures ${ }^{32}$.

The effect of molecular weight on the zero shear viscosity is found to obey the 3.4 power law although some researchers have reported a 4.2 power. For the 3.4 power law 
case, the effect of molecular weight on the shear viscosity can be expressed as ${ }^{33}$ :

$$
\eta_{0}=1.5 \rho \mathrm{T} \lambda
$$

where

$$
\lambda=1.4 \mathrm{X} 10^{-21} \mathrm{M}_{\mathrm{w}}^{3.4} \exp \left(\frac{1}{\beta\left(\mathrm{T}-\mathrm{T}_{\infty}\right)}\right)
$$

and

$\beta=8 \times 10^{-4} \mathrm{~K}$

Table 2-6 lists the shear rate for polymer processing. one need to measure the viscosity of polycarbonate in shear rates between 10 and $15000 \mathrm{~s}^{-1}$, which is the same shear rate in extrusion and injection molding processes.

Table 2-7 Typical shear rate ranges for polymer processing.

\begin{tabular}{ll}
\hline \hline Process & Shear rate rang, $\mathrm{s}^{-1}$ \\
\hline Compression molding & $1-10$ \\
Calendaring & $10-100$ \\
Extrusion in barrel & $10-400$ \\
Extrusion in die & $50-15,000$ \\
Injection molding & $500-15,000$ \\
Blow molding & $100-10,000$ \\
\hline \hline
\end{tabular}




\subsubsection{Stability}

PC is normally fabricated at high melt temperatures $\left(300^{\circ} \mathrm{C}\right.$ and above) where yellowing and molecular weight degradation can occur in the presence of impurities such as water, oxygen, and iron ${ }^{34}$. It is recommended that PC be dried to below $200-500 \mathrm{ppm}$ of water to prevent a decrease in toughness ${ }^{35}$. Thermal-oxidative stabilizers for PCs are usually hindered phenols, and phosphites/phosphonites ${ }^{\mathbf{3 6}}$.

Under prolonged exposure to UV light, PC tends to yellow and degrade due to a photooxidation reaction ${ }^{37}$. For outdoor applications, $\mathrm{PC}$ is normally protected by adding a UV stabilizer which protects the PC by selective absorption of the harmful UV radiation. Common UV stabilizers employed are benzotriazoles ${ }^{38}$.

In high humidity and high temperature applications, the hydrolytic stability of PC becomes important. PC has an equilibrium moisture content of $0.34 \%$ at $25^{\circ} \mathrm{C}$ and $0.48 \%$ at $100^{\circ} \mathrm{C}$ and $100 \%$ relative humidity. Impurities in PCs may, however, react with water and cause degradation of the mechanical properties. Certain additives may also accelerate the hydrolytic degradation of $\mathrm{PC}$ at temperatures above $60^{\circ} \mathrm{C}$. This process will eventually decrease both notched Izod impact strength and molecular weight ${ }^{39}$.

\subsubsection{Processing of polycarbonates}

Polycarbonates can be processed using conventional methods such as extrusion, blow molding, injection molding, and solution casting. PCs with molecular weights in the range of less than $35,000 \mathrm{~g} / \mathrm{mol}$ are commonly used in injection molding applications. For blow molding applications, due to the need of melt strength, a PC with a molecular 
weight of more than $32,000 \mathrm{~g} / \mathrm{mol}$ is normally used. PC needs to be dried prior to processing to prevent degradation and foaming. Normally PC is dried using a convective dryer or oven at about $120{ }^{0} \mathrm{C}$ for at least 4 hours. The drying step can sometimes be bypassed if the PC is processed using a vented extruder ${ }^{40}$. The normal barrel set temperature for processing PC by an extruder or an injection molding ranges from $250{ }^{\circ} \mathrm{C}$ to $300{ }^{\circ} \mathrm{C}$.

\subsection{Applications of polycarbonates}

Polycarbonate, with its combined properties of transparency, toughness, and high heat resistance, has numerous applications that range from use in the manufacturing of small parts such as fuses to large sheets used in construction. Below are some important applications that capitalize on key properties.

\subsubsection{Optics}

Surface coatings on optical information media such as digital audio compact disk (CD) and compact disk read-only memory (CD-ROM) are most often made of PC, as shown in Figure 2-11. 


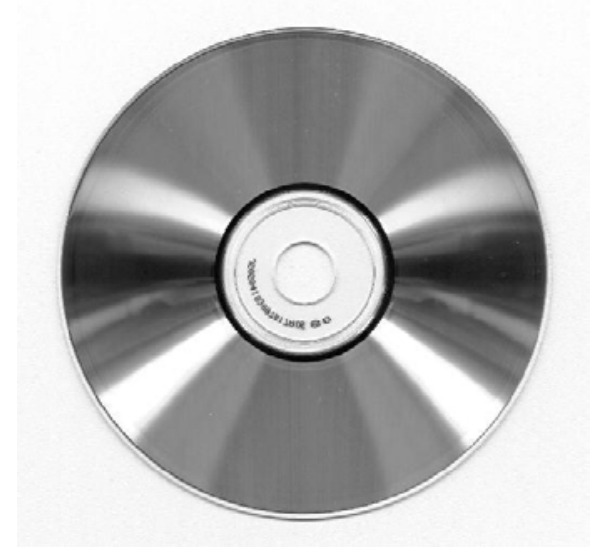

Figure 2-11 A compact disk made from Polycarbonate

In order to be suitable for use as a substrate, the PC must be of superior quality with a transparency of greater than $87 \%$ spectral light transmission for a 4 -mm-thick sheet, colorless with requirements of a yellow index less than 4.5. PC is also used extensively in optical applications such as lenses ${ }^{41}$, for camera components, goggles and safety glasses. The combination of light weight and high resistance to impact makes PC an ideal substitute for glass in these applications. PC must, however, be rendered scratch resistant for optical use by the addition of a coating such as acrylate or silicate.

\subsubsection{Construction and housing}

PC is widely used to fabricate sheets for the construction industry. Applications for these sheets include windows, laminated walls, infrared reflective insulation, and skylight roofing. PC sheets can also be used in the fabrication of household goods such as tables and desktops. For these applications a scratch-resistent coating is often applied to render the product more durable. When manufactured for use outdoors, a UV-resistant coating or a UV-resistant additive is desirable to prevent the PC from breaking down or 
yellowing in sunlight. It is also possible to change the composition of the PC to make it opalescent for use in light-diffusing roof covers and lighting components.

\subsubsection{Automotive}

PC is an ideal material for use in many automotive applications which undergo constant and heavy use. Components such as speedometer needles, windscreens, instrument panels, headlamp covers and housings, and toolboxes can all be made from PC. Because of the particular fire and impact hazards associated with automotive use, for these applications, PC must be rendered nonflammable and strengthened by ignitionresistant additives and impact modifiers. Exposure to sunlight also means that UVresistant additives must be included. The PC may also be adapted so that it can be painted or will adhere to parts made of other materials.

\subsubsection{Medical devices}

When used in dental or medical supplies ${ }^{\mathbf{4 2}}$, such as containers, disposable syringes, and various kinds of tubing, including that used for kidney dialysis, the PC must be able to withstand autoclave temperatures of $134{ }^{0} \mathrm{C}$ for sterilization.

\subsubsection{Appliances and consumer products}

PCs are used extensively in the manufacture of domestic products such as dinnerware, drinking cups, and toys. These products can be either transparent or colored 
and must have Food and Drug Administration approval for their use. Products in this category are made using blow molding techniques, which require high molecular weights and/or branched PCs with high melt strength.

PCs are also used in the manufacture of appliances and other household articles that require heat-, ignition-, and impact-resistant qualities. Some of these are refrigerator housings, dishwasher trays and even eyelets for shower curtains ${ }^{43}$. Polycarbonate is also used extensively in the home and the office in the manufacture of telephones, fax machines and computer housing - all applications in which degree of wear requires the material to be highly durable.

\subsection{Modification of polycarbonates}

Relatively few polymers that are produced today are used in their pure form. Most contain, or are combined with, other materials for various reasons. Some of these are: ${ }^{44}$

- To improve mechanical properties such as modulus, strength, hardness, abrasion resistance, and toughness.

- To prevent degradation (both during fabrication and in service).

- To change the thermal properties, such as the expansion coefficient and the conductivity.

- To reduce materials costs.

- To improve the processability.

Different kinds of materials are mixed with polymers for various reasons; some are called "additives". Additives are solids or liquids used primarily as colorants, UV 
absorbers, plasticizers, flame-retardants, thermal stabilizers, lubricants, and antistatic agents. The resulting mixture can be either homogeneous or heterogeneous, depending on the solubility of the additive in the polymer. Other kinds of materials that are mixed with polymers include a variety of particulate and fibrous materials which act as fillers and reinforcement. The result is typically a heterogeneous mixture; that is, because these materials do not dissolve in the polymer, the result is a multiphase system. Fibers are materials with lengths many times their thickness and widths. Some that are frequently mixed with polymers are ${ }^{45}$ :

- Glass fibers

- Carbon fibers

- Organic polymeric fibers

- Mineral fibers

- Metal fibers

- Ceramic fibers

Fibers are added to polymers for a variety of reasons, one of which is to increase their strength. The addition of fibers does not, however, always imply increased strength. Additives, fillers, or reinforcing agents such as fibers, may be mixed with a polymer to increase its utility and range of application. Any added material does, however, make analysis of the polymer more difficult because it is subject to all of the variables implied in the use of any added materials. Other variables, such as those listed below, exist when a fibrous material is embedded in a polymer matrix. They are:

- The properties of the fiber (density, hardness, strength, etc.)

- The size and shape of the fiber 
- The amount of fiber added

- The alignment of the fiber

- The distribution of the fiber lengths and orientations

- The nature of the interface between the polymer and the fiber

\subsubsection{Glass fibers}

Many different compositions of mineral glasses have been used to produce fibers. The most common are based on silica $\left(\mathrm{SiO}_{2}\right)$ with the additions of oxides of calcium, boron, sodium, iron and aluminum. These glasses are usually amorphous. Typical compositions of the three well-known glasses used for glass fiber in composite are given in Table 2-8.

Table 2-8 Composition of glass used for fiber manufacture (value are in $\mathrm{wt} \%$ )

\begin{tabular}{llll}
\hline \hline & E glass & C glass & S glass \\
\hline $\mathrm{SiO}_{2}$ & 52.4 & 64.4 & 64.4 \\
$\mathrm{Al}_{2} \mathrm{O}_{3}, \mathrm{Fe}_{2} \mathrm{O}_{3}$ & 14.4 & 4.1 & 25.0 \\
$\mathrm{CaO}$ & 17.2 & 13.4 & --- \\
$\mathrm{MgO}$ & 4.6 & 3.3 & 10.3 \\
$\mathrm{Na}_{2} \mathrm{O}, \mathrm{K}_{2} \mathrm{O}$ & 0.8 & 9.6 & 0.3 \\
$\mathrm{Ba}_{2} \mathrm{O}_{3}$ & 10.6 & 4.7 & --- \\
$\mathrm{BaO}$ & --- & 0.9 & -- \\
\hline \hline
\end{tabular}


E glass is the most commonly used because it draws well and its strength, stiffness, electrical and weathering properties are superior. $\mathrm{C}$ glass is more resistant to chemical corrosion than $\mathrm{E}$ glass but is more expensive and less durable. $\mathrm{S}$ glass is more expensive than E glass but has a higher Young's modulus and is more temperature resistant.

\subsubsection{Fiber length distributions}

Fiber length distributions in composite plastics, where short fibers are used, arise because the fibers may be fractured by the extrusion process. This affects the viability of the fibers, so attention must be paid at the outset of the process to ensure correct fiber length distribution, an important factor in the successful manufacture of short-fiber composites. Distribution of fiber-length is determined through one of two possible methods. A measurement can be taken of some physical property of the composite that is dependent on the length of the fibers, such as strength or modulus. This is known as the "indirect" method, and it achieves less precise results than the second, or "direct" method in which the matrix is dissolved or burned, thus separating it from the fibers.

The definition of a meaningful average fiber length is difficult, but two simple averages are commonly used ${ }^{46}$.

The number average fiber length $\left(\mathrm{L}_{\mathrm{N}}\right)$ is defined as:

$$
\mathrm{L}_{\mathrm{N}}=\frac{\sum \mathrm{N}_{\mathrm{i}} \mathrm{L}_{\mathrm{i}}}{\sum \mathrm{N}_{\mathrm{i}}}
$$

where $\mathrm{N}_{\mathrm{i}}$ is the number of fibers of length $\mathrm{L}_{\mathrm{i}}$

The weight or volume average fiber length $\left(\mathrm{L}_{\mathrm{W}}\right)$ is defined as: 


$$
\mathrm{L}_{\mathrm{W}}=\frac{\sum \mathrm{W}_{\mathrm{i}} \mathrm{L}_{\mathrm{i}}}{\sum \mathrm{W}_{\mathrm{i}}}
$$

where $\mathrm{W}_{\mathrm{i}}$ is the weight of fibers of length $\mathrm{L}_{\mathrm{i}}$. For fibers of constant diameter, the weight average fibers length can be expressed as:

$$
\mathrm{L}_{\mathrm{w}}=\frac{\sum \alpha \mathrm{N}_{\mathrm{i}} \mathrm{L}_{\mathrm{i}}^{2}}{\sum \alpha \mathrm{N}_{\mathrm{i}} \mathrm{L}_{\mathrm{i}}}=\frac{\sum \mathrm{N}_{\mathrm{i}} \mathrm{L}_{\mathrm{i}}^{2}}{\sum \mathrm{N}_{\mathrm{i}} \mathrm{L}_{\mathrm{i}}}
$$

where $\alpha=\pi \mathrm{r} 2 \rho, \mathrm{r}$ fiber radius and $\rho$ fiber density

\subsubsection{Fiber orientation}

The processing of short-fiber composite material often changes the orientation of the fibers. These changes are represented in a two-dimensional deformation in Figure 212. When the fibers rotate towards the direction of the extension, it is known as elongation or extensional flow. With large extensions, a high degree of alignment may be expected. Shear flow is when some of the fibers rotate towards the direction of shear and others rotate in the opposite direction, so that there is no net change in orientation. Thus, the degree of preferred fiber orientation after processing depends on the flow field. The viscosity of the matrix affects the final orientation distribution mainly through its effect on the way in which the mold fills ${ }^{47}$. 


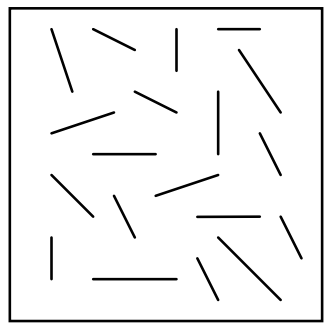

(a)

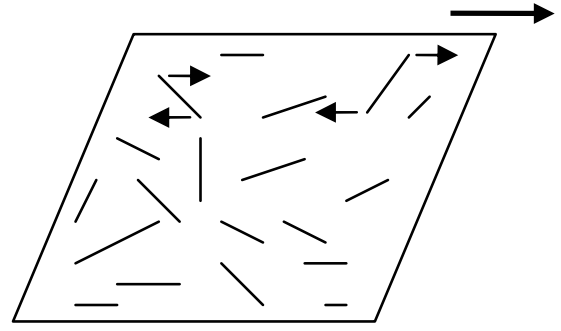

(b)

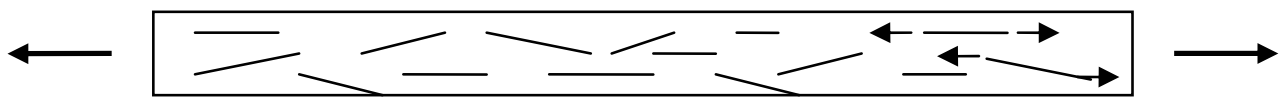

(c)

Figure 2-12 Schematic presentation of the changes in fiber orientation occurring during flow. (a) Initial random distribution, (b) rotation during shear flow and (c) alignment during elongation flow

\subsection{Fiber reinforced thermoplastics}

For continuous and unidirectional fiber composites, stiffness is reasonably well predicted by the "Rule of Mixtures" (ROM), which is based on a parallel model for tension specimens tested in the direction of fiber alignment. In this model, the modulus of composites, $\mathrm{E}_{\mathrm{c}}$, is given by

$$
E c=E_{f} v_{f}+E_{m}\left(1-v_{f}\right)
$$

where $E_{m}$ and $E_{f}$ are the elastic moduli of the matrix and the fiber respectively and $v_{f}$ is the fiber volume fraction. The above equation assumes the Poisson's ratio of the fiber and 
matrix to be equal.

ROM is not as successful a predictor of the strength of continuous fiber composites, and experimental results do not match those predicted by ROM. Most fibers used in thermoplastics reinforcers have a much smaller degree of elongation than the matrix. The largest stress ever experienced by the matrix at fracture would then be a smaller value $\left(\sigma_{\mathrm{m}}^{\prime}\right)$ than the tensile strength of the matrix $\left(\sigma_{\mathrm{m}}\right)$. This was first proposed by Kelly and Davies ${ }^{48}$ and is now known as the "Modified Rule of Mixtures" (MROM). The strength of the continuous fiber composites is given by Modified Rule of Mixtures as

$$
\sigma_{\mathrm{c}}=\sigma_{\mathrm{f}} \mathrm{v}_{\mathrm{f}}+\sigma_{\mathrm{m}}\left(1-\mathrm{v}_{\mathrm{f}}\right)
$$

where $\sigma_{\mathrm{c}}$ and $\sigma_{\mathrm{f}}$ are the tensile strength of the composite and the fiber respectively and $\mathrm{v}_{\mathrm{f}}$ $>\mathrm{v}_{\mathrm{fc}}$. In order to fully utilize the capacity of the matrix, the volume fraction of fibers introduced needs to exceed a critical value, $\mathrm{v}_{\mathrm{fc}}$.

A phenomenon known as "shear-lag" analysis, which was first reported by $\mathrm{Cox}^{49}$, is essentially the transfer of a load applied in the direction of the fibers contained in a fiber-reinforced composite. The transfer agents are the shear stresses at the interface of the composite. This same process is also applicable to composites reinforced by short fibers.

Equation (2-11) may be modified by including a length correction factor, $\eta_{\mathrm{L}}$, for uniaxially aligned fibers of finite length:

$$
E_{c}=E_{f} v_{f} \eta_{L}+E_{m}\left(1-v_{f}\right)
$$


This length correction factor is given by Cox as:

$$
\eta_{\mathrm{L}}=1-\left[\frac{\tanh (\beta \mathrm{L} / 2)}{(\beta \mathrm{L} / 2)}\right]
$$

where $L$ is the fiber length and $\beta$ is given by

$$
\beta=\left[\frac{2 \pi \mathrm{G}_{\mathrm{m}}}{\mathrm{E}_{\mathrm{f}} \mathrm{A}_{\mathrm{f}} \ln (\mathrm{R} / \mathrm{r})}\right]^{1 / 2}
$$

In this equation, $G_{m}$ is the matrix shear modulus, $A_{f}$ the cross-sectional area of the fiber, $\mathrm{r}$ the fiber radius and $\mathrm{R}$ the mean separation of fibers normal to their length. Cox assumed that in order to derive the length correction factor, the fibers were arranged hexagonally, and this distribution was uniform throughout the cross-section of the composite. It can be seen, from the above equations, that the value of $\beta$ may be evaluated given values for $G_{m}, E_{f}, A_{f}$, and $V_{f}$. It can easily be shown by the following relation that, for hexagonal distribution of fibers, volume fraction of fibers can be calculated in terms of $r$ and $R$ :

$$
\mathrm{v}_{\mathrm{f}}=\frac{2 \pi \mathrm{r}^{2}}{\sqrt{3} \mathrm{R}^{2}}
$$

The magnitude of $\beta$ will essentially determine the "scale" of the dependence of $E_{c}$ on fiber length (L). For instance, it will directly affect the length of the fiber required to give a value of $E_{c}$ close to that expected for a continuous fiber composite. It is extremely important in this study that we understand the predicted dependence of $E_{c}$ on fiber length for short fiber reinforced thermoplastics (SFRTP). 
We should be aware of certain assumptions that are evident in Cox's analysis. First, the matrix and fibers are assumed to be strained elastically, and second, there is no relative movement at the matrix interface under applied loads, which means that the displacements of the fiber, matrix and overall composite are identical during loading $\left(\mathrm{e}_{\mathrm{f}}=\right.$ $\mathrm{e}_{\mathrm{m}}=\mathrm{e}_{\mathrm{c}}$ ). Finally, the fibers are assumed to be laid out in a parallel configuration and are aligned unidirectionally in the intended loading direction.

\subsubsection{The stiffness of partially oriented composites}

Control of fiber orientation during production of short-fiber reinforced thermoplastics is not possible when common production techniques are used. We have concentrated the discussions on the properties of uniaxially aligned short fiber composites. In order for the theory to have any practical application, however, we must account, in the theoretical analyses, for the fiber orientation (FOD).

$\mathrm{Krenchel}^{35}$ developed an "orientation efficiency factor" in order to account for the anisotropy of stiffness in Cox's equation:

$$
\mathrm{E}_{\mathrm{c}}=\mathrm{E}_{\mathrm{f}} \mathrm{v}_{\mathrm{f}} \eta_{\mathrm{L}} \eta_{\mathrm{o}}+\operatorname{Em}\left(1-\mathrm{v}_{\mathrm{f}}\right)
$$

The orientation efficiency factor $\eta_{\mathrm{o}}$ is determined by dividing the reinforcement into groups of uniaxially aligned fibers:

$$
\eta_{\mathrm{o}}=\sum_{\mathrm{h}} \mathrm{a}_{\mathrm{h}} \cos ^{4} \theta_{\mathrm{h}}
$$

where $\sum_{\mathrm{h}} \mathrm{a}_{\mathrm{h}}=1$ 
and $a_{h}$ is the fiber fraction oriented at an angle $\theta_{h}$ with respect to the applied load.

It should be noted that $\eta_{\mathrm{o}}$ does not give a unique representation of the fiber orientation distribution. Nonetheless, it is a useful indicator of the overall degree of fiber alignment. The use of Equation (2-13) represents a very simple method for the prediction of the tensile stiffness of SFRTP materials. However, this equation predicts that fibers lying transverse to the direction of applied stress offer no reinforcement to the matrix.

\subsubsection{Strength of short-fiber composites}

The variety of industrial uses for short-fiber reinforced thermoplastics, and the necessity to ensure that their strength is equal to the proposed use, require that it must be possible to predict the fiber strength. This, however, is a complex process. There is always a possibility of the composite being unable to withstand a load when the material contains unidirectionally aligned continuous fibers. The failure can occur in various places: in the fibers, the matrix or at the fiber-matrix interface. This may happen even when the tensile stress is applied along the fiber axis, if the bond between the fibers and matrix is not of the required strength. If we assume that the bond between the fiber and matrix is a strong one, the strength of the unidirectionally aligned continuous fiber composite is given by Equation (2-12), as mentioned earlier. In reality however, this equation does not accurately describe the strength of a continuous fiber reinforced composite. Furthermore, in the case of a composite containing short fibers, the existence of a non-uniform stress along the fibers implies that the average stress $\bar{\sigma}_{f}$ carried by fibers at failure will be less than the maximum strength of the fibers $\sigma_{\mathrm{f}}$ which is given in 
Equation (2-12). The actual relationship connecting $\bar{\sigma}_{\mathrm{f}}$ and $\sigma_{\mathrm{f}}$ will of course depend on the exact form of the stress distribution at the fiber ends. This relationship was established in the Cox analysis for the case of an elastic matrix and elastic fibers. With reference to Figure 2-13 consider a fiber of length $L$ and radius $r$ embedded in a matrix. The relationship as follows:

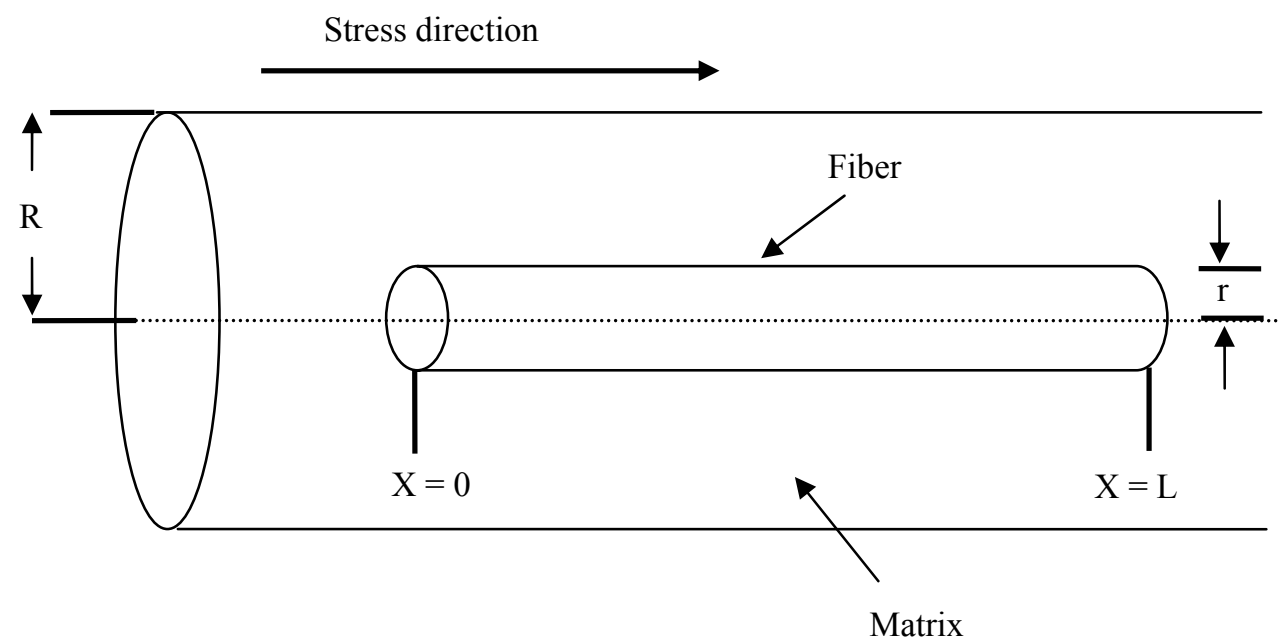

Figure 2-13 Definition of symbols used in Cox shear-lag analysis

$$
\begin{aligned}
& \sigma_{\mathrm{f}}=\mathrm{E}_{\mathrm{f}} \mathrm{e}\left[1-\frac{\cosh \beta\left(\frac{\mathrm{L}}{2}-\mathrm{x}\right)}{\cosh \left(\beta \frac{\mathrm{L}}{2}\right)}\right] \\
& \bar{\sigma}_{\mathrm{f}}=\mathrm{E}_{\mathrm{f}} \mathrm{e}\left[1-\frac{\tanh \beta \frac{\mathrm{L}}{2}}{\beta \frac{\mathrm{L}}{2}}\right]
\end{aligned}
$$

where $\beta$ was given for the Equation (2-13). The average longitudinal stress in a short fiber reinforced composite $\left(\sigma_{c}\right)$ is given in the Cox analysis as a weighted average of the 
stresses developed separately in the fiber and matrix:

$$
\sigma_{\mathrm{c}}=\bar{\sigma}_{\mathrm{f}} \mathrm{v}_{\mathrm{f}}+\sigma_{\mathrm{m}}^{\prime}\left(1-\mathrm{v}_{\mathrm{f}}\right)
$$

\subsubsection{Strength of partially oriented composites}

As in the case of stiffness, the strength of an aligned short fiber composite decreases as the angle between the fiber axis and the loading direction increases. Actually, when the load is applied transversely, the strength of the composite can sometimes be less than the matrix, due to the stress raising effect of the fibers. The prediction of the effect of anisotropy on strength requires attention. The first study of this type was introduced by Stowell and $\mathrm{Liu}^{\mathbf{5 0}}$. They analyzed maximum stress criterion in three regions with different failure mechanisms:

- For small angles $\theta$ between loading direction and fiber axis, failure is controlled by the fiber strength and the suggested mathematical expression for the tensile fiber failure is:

$$
\sigma_{\mathrm{c} \theta}=\sigma_{\mathrm{c}} \sec ^{2} \theta
$$

- At larger angles of $\theta$, shear stresses developing both in the matrix and along the fibermatrix interface increase and the dominant failure mode becomes due to shear processes:

$$
\sigma_{\mathrm{c} \theta}=2 \tau_{\mathrm{c}} \sec ^{2} \theta
$$

for shear failure parallel to the fibers, where $\tau_{\mathrm{c}}$ is the in-plane composite shear strength.

- At very large $\theta$, approaching $90^{\circ}$, the mode changes again to one of transverse failure 
either in the matrix or at the interface. Then:

$$
\sigma_{\mathrm{c}}=\sigma_{\mathrm{ct}} \operatorname{cosec}^{2} \theta
$$

for tensile failure normal to the fibers. Here, $\sigma_{\mathrm{ct}}$ is the transverse composite strength.

Table 2-9 lists typical properties of glass-reinforced polycarbonate. It can be seen that when adding glass fiber to polycarbonate the strength and stiffness increases while the impact strength and elongation decreases.

Table 2-9 Typical properties of glass reinforced polycarbonate (LEXAN is manfuactured by GE plastics)

\begin{tabular}{|c|c|c|c|c|c|}
\hline Property & ASTM & $\begin{array}{l}\text { LEXAN }^{\circledR} \\
\text { HF1110 } \\
\end{array}$ & $\begin{array}{c}\text { LEXAN }^{\circledR} \\
500 \\
\end{array}$ & $\begin{array}{c}\text { LEXAN }^{\circledR} \\
3412 \mathrm{R} \\
\end{array}$ & $\begin{array}{c}\text { LEXAN }^{\circledR} \\
3412 \mathrm{R} \\
\end{array}$ \\
\hline Glass Fiber, wt $\%$ & & 0 & 10 & 20 & 30 \\
\hline Tensile Strength, break psi & D-638 & 9500 & 8000 & 16000 & 14500 \\
\hline $\begin{array}{l}\text { Tensile Elongation, } \\
\text { break } \%\end{array}$ & D-638 & 120 & 8 & 5 & 2 \\
\hline Flexural Stress, yield psi & D-790 & 13500 & 15000 & 19000 & 22200 \\
\hline Flexural Modulus,psi & D-790 & 335000 & 500000 & 800000 & 960000 \\
\hline $\begin{array}{l}\text { Izod Impact, } \\
\text { notched ft-lb/in }\end{array}$ & D-265 & 12 & 2 & 2 & 2 \\
\hline
\end{tabular}

\subsubsection{Fiber suspensions}

A fiber suspension is a liquid mixture in which glass, nylon, graphite, or mineral fibers are dispersed. The suspending liquid may be a polymer solution or a melt. The presence of the fibers and their orientation changes the viscosity, normal stress, and other characteristics of the flow. Fiber direction is a result of the shear deformation, and the 
function of fiber orientation depends upon the concentration of fibers, the fiber aspect ratio, and the deformation gradients. The criterion for dilute and non-dilute fiber suspensions is the volume fraction of fibers $(\phi)$. The ranges of glass fibers concentrations are given below:

$$
\begin{array}{ll}
\text { Dilute: } & 0<\phi<(\mathrm{D} / \mathrm{L})^{2} \\
\text { Semi-concentrated: } & (\mathrm{D} / \mathrm{L})^{2}<\phi<(\mathrm{D} / \mathrm{L}) \\
\text { Concentrated: } & (\mathrm{D} / \mathrm{L})<\phi
\end{array}
$$

Here $\mathrm{D}$ is the fiber diameter and $\mathrm{L}$ is the fiber length. Note that the volume fraction, $\phi$, and aspect ratio, (L/D), are the dimensionless quantities that characterize the suspension. The spacing between particles is a very important parameter. For a given fiber-volume fraction the spacing between fibers depends upon the fiber aspect ratio and the fiberorientation distribution function. The spacing between fibers, $\mathrm{h}$, is defined as $(\mathrm{nL})^{-0.5}$ for an aligned initial fiber orientation ${ }^{51}$, or $(\mathrm{nL})^{-1}$ for a random state ${ }^{52}$. Figure 2-14 shows different fiber configurations. In the dilute regime, $\phi<(D / L)^{2}$ the distance between a fiber and its nearest neighbor is greater than $\mathrm{L}$. The fibers are free to rotate and the interactions between fibers are rare. For fibers with large aspect ratio the volume fraction must be quite small for the suspensions to be dilute. Most commercial composites have glass loadings more than 0.10 volume fraction, and are in the semi-concentrated and concentrated regimes. 


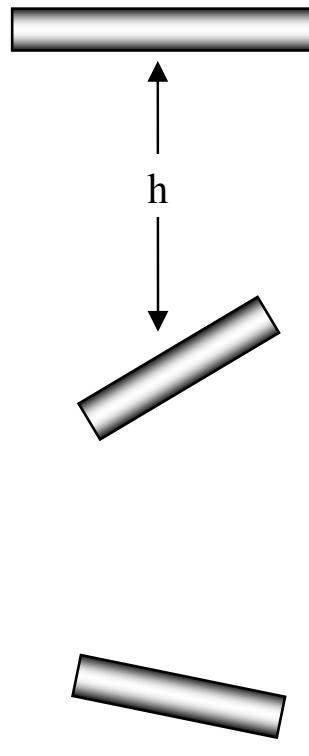

Dilute $h>L$
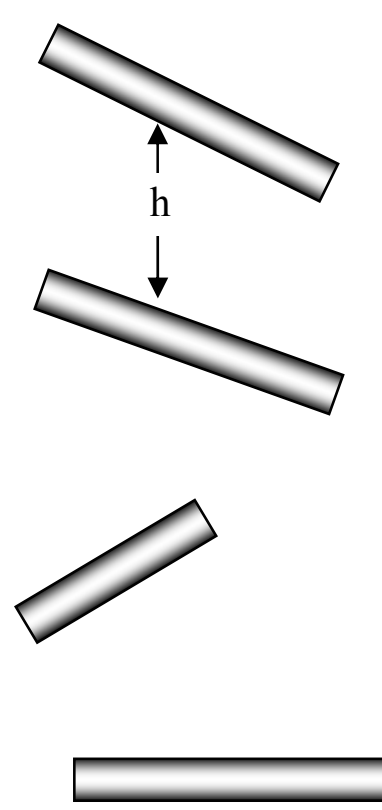

Semi-concentrated $\mathrm{D}<\mathrm{h}<\mathrm{L}$

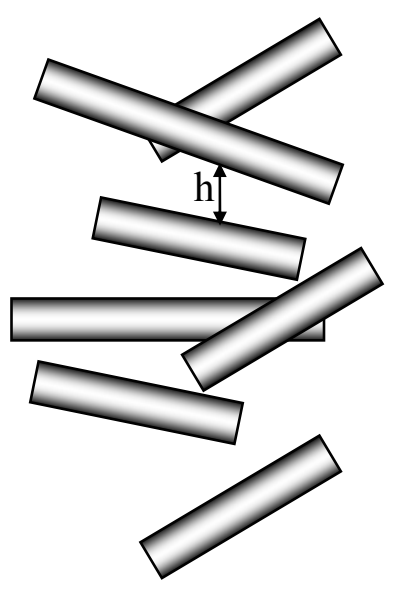

Concentrated $\mathrm{h}<\mathrm{D}$

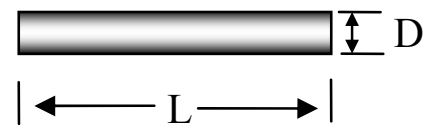

Figure 2-14 Fiber spacing for dilute, semi-concentrated, and concentrated fibers

If the volume fraction of fibers falls within the semi-concentrated range, the spacing between the fibers is less than L but greater than D, and interactions between fibers are frequent. The highly concentrated regime denotes spacing between fibers on the order of D.

The motion of particles in a viscous medium has been the subject of many studies. The increased viscosity of fiber suspensions has been theoretically modeled and 
experimentally investigated by a host of researchers ${ }^{53}$. The theoretical work predicts an increase in viscosity due to the addition of spheres, fibers, and other particles to Newtonian solvents.

The first successful viscosity equation, proposed by Einstein, linearly related the reduced viscosity to the volume fraction of spheres by considering the energy dissipation from the fibers to the bulk fluid. Extensions of the Einstein equation were developed for uniform solid spheres ${ }^{54}$, suspensions of non-spherical particles in high-viscosity polymer fluids ${ }^{55}$, and in the semi-concentrated and concentrated region of fiber suspensions ${ }^{56}$. A listing of the empirical equations is given in Table 2-10.

Table 2-10 Empirical viscosity equations

\begin{tabular}{|ll|} 
Einstein & $\eta_{\mathrm{r}}=1+2.5 \phi$, where $\eta_{\mathrm{r}}=\frac{\eta_{\mathrm{s}}}{\eta_{\mathrm{o}}}$ \\
Thomas & $\eta_{\mathrm{r}}=1+2.5 \phi+10.05 \phi^{2}+\mathrm{A} \exp (\mathrm{B} \phi)$ \\
Mooney & $\eta_{\mathrm{r}}=\exp \left(\frac{2.5 \phi}{1-\mathrm{k} \phi}\right)$ \\
Zeigal & $\eta_{\mathrm{r}}=2.5 \phi\left(1+\frac{\mathrm{r}}{\mathrm{R}_{0}}\right)^{3}$ \\
Frankel & $\eta_{\mathrm{r}}=\frac{9}{8} \frac{\left(1+\frac{\phi}{\phi_{\mathrm{n}}}\right)^{1 / 3}}{\left(1-\frac{\phi}{\phi_{\mathrm{n}}}\right)^{1 / 3}}$ \\
Maron and Pierce & $\eta_{\mathrm{r}}=\left[1-\left(\frac{\phi}{\mathrm{A}^{*}}\right)\right]^{2}$ \\
\hline
\end{tabular}

Here, $\phi$ is the volume fraction of particles, $\eta_{\mathrm{r}}$ is the reduced viscosity, and $\mathrm{r}$ is the radius of the particle. A, $\mathrm{A}^{*}, \mathrm{~B}, \mathrm{k}, \mathrm{R}$ and $\phi_{\mathrm{n}}$ are empirical constants. The empirical models are limited to Newtonian solvents and dilute suspensions. More importantly, they do not 
offer a complete description of the stress field caused by the presence of the particles. This limitation prevents the use of the viscosity expressions in the flow equations.

Previous experimental work covered a wide range of volume fractions, aspect ratios, fiber types, and polymer types. Most of the published work involved dilute-fiber suspensions ${ }^{57,58}$. An excellent review of particle suspensions in Newtonian and nonNewtonian solvents is provided elsewhere ${ }^{59}$. Figure 2-15 shows the apparent viscosity of LEXAN HF1110 (polycarbonate) and LEXAN 500 (polycarbonate with 10\% glass fibers). The viscosity is measured by GE Plastics using a capillary viscometer at a temperature equal to $280{ }^{\circ} \mathrm{C}$.

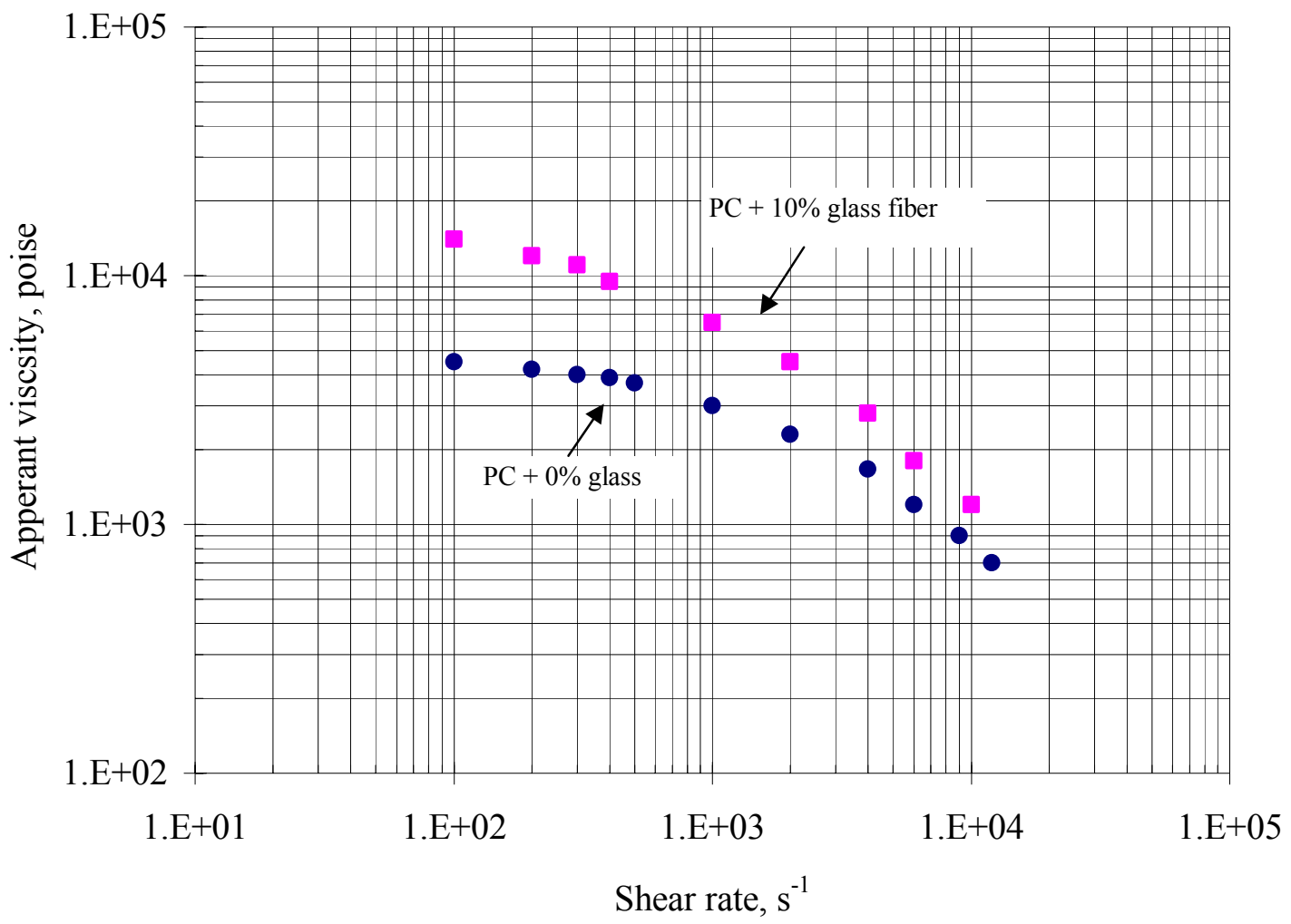

Figure 2-15 Viscosity of un-reinforced polycarbonate (LEXAN ${ }^{*}$ HF1110) and glass reinforced polycarbonate (LEXAN* 500$)$. Taken from www.GEplastics.com. 


\subsubsection{Dynamic mechanical analysis}

Dynamic mechanical analysis (DMA), one of the most powerful tools available for the study of the behavior of plastic materials, is used to measure their viscoelastic properties. Since all polymers are viscoelastic in nature, DMA is ideal for performing evaluations on the complex array of phenomena that characterize polymeric materials. Most classical materials respond to applied stress with either elastic or viscous behavior. Elastic responses, manifested as a proportional deformation by a quantity known as a strain, are typically seen in solid materials. Viscous behavior, a characteristic of fluid materials, is manifested as a strain that increases proportionally with time until the stress is removed ${ }^{60}$.

DMA test provides valuable information, the most readily accessible of which is the plot of storage modulus versus temperature (see Figure 2-16). With this information, we are able to determine the basic structure - that is, distinguish between a semicrystalline and an amorphous material —of the polymer system. The storage modulus indicates the viscous nature of the polymer and is affected by fibers in a similar manner to the shear viscosity (storage modulus increases as the fiber content increases as seen in Figure 2-17). The loss modulus indicates the viscous nature of the polymer and gives information about viscous flow or energy dissipation during flow. The fibers will affect the loss modulus in a similar fashion to the storage modulus (see Figure 2-18). The fibers at low frequency increase the loss modulus, while at high frequency the fibers become aligned and contribute less to loss modulus ${ }^{61}$. 


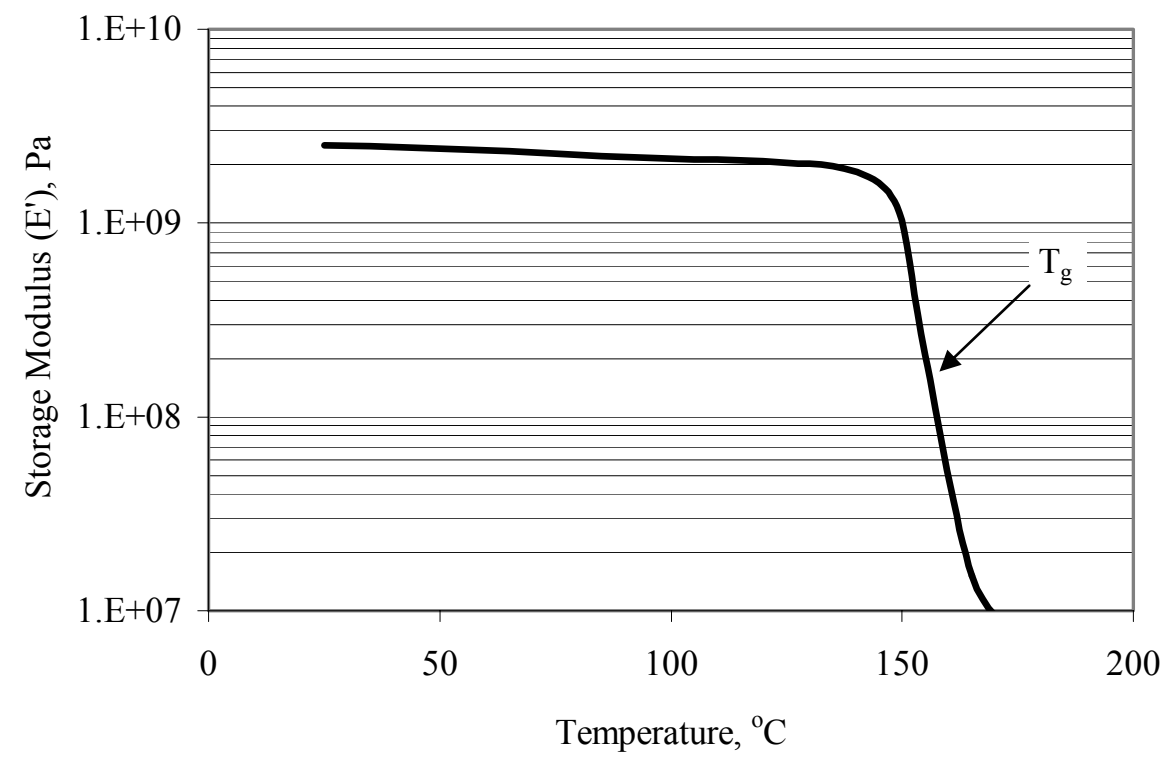

Figure 2-16 Storage modulus (E') for Polycarbonate LEXAN HF1110 (test conducted at frequency equal to $1 \mathrm{~Hz}$ )

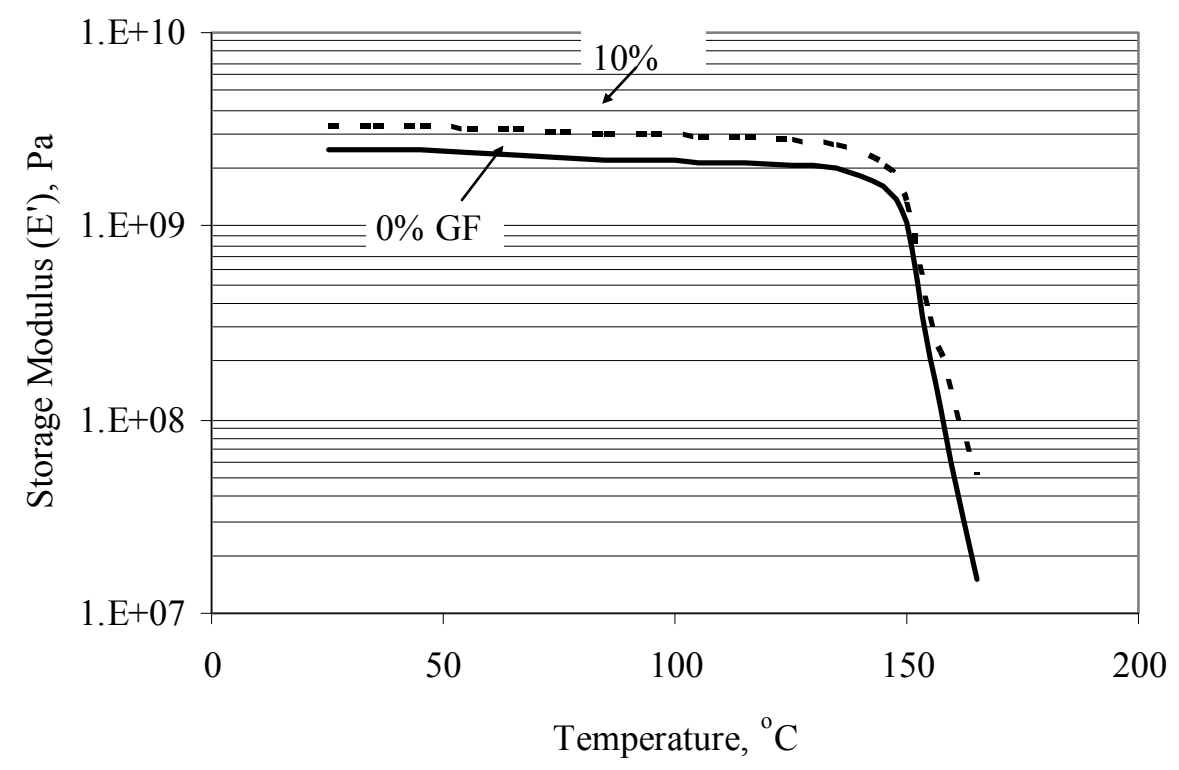

Figure 2-17 Storage modulus for un-reinforced polycarbonate and 10\% glass reinforced polycarbonate (test conducted at frequency equal to $1 \mathrm{~Hz}$ ) 


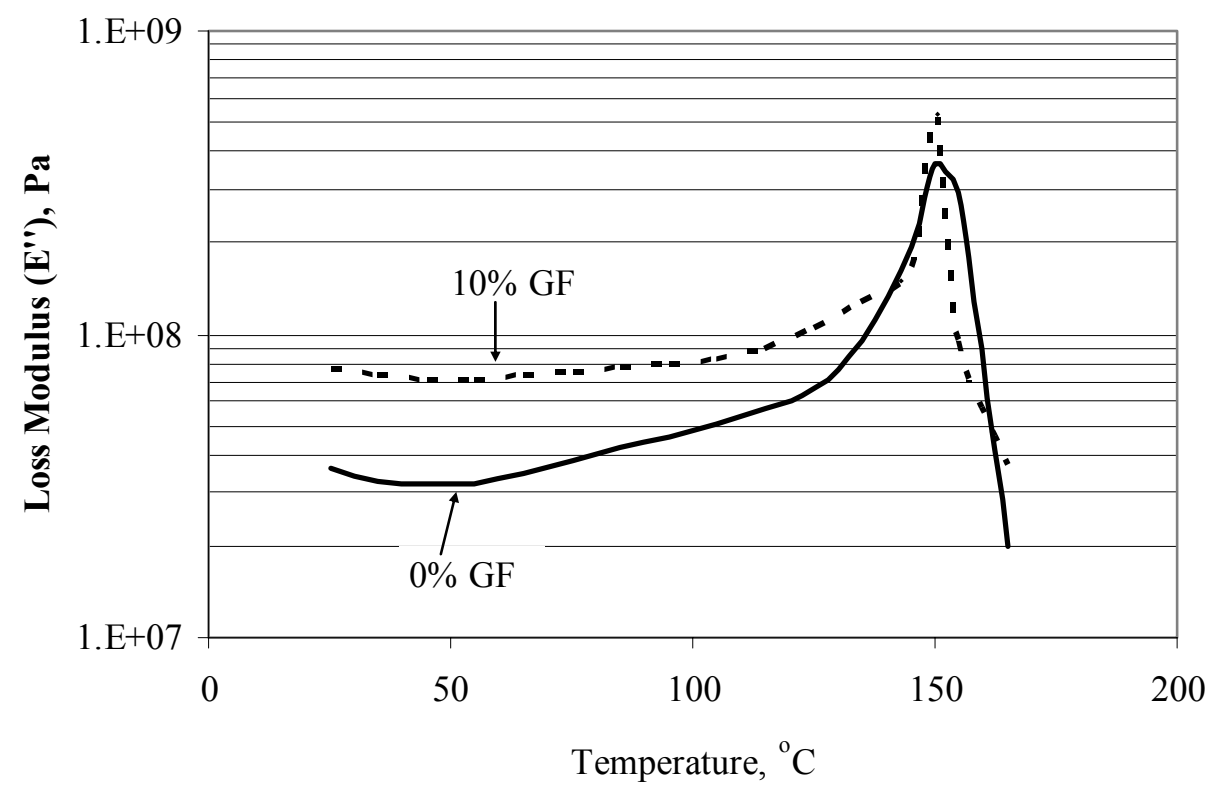

Figure 2-18 Loss modulus for un-reinforced polycarbonate and 10\% glass reinforced polycarbonate (test conducted at frequency equal to $1 \mathrm{~Hz}$ ) 


\section{Purpose of this Study and Approach Taken}

This study aims to determine how the mechanical and rheological properties of recycled polycarbonate can be improved (see Figure 3-1) and to examine the effects of the presence of impurities on un-reinforced virgin and glass reinforced virgin polycarbonate. For the purpose of this research, recycled polycarbonate is defined as polymer which has been separated from other plastics. After separation, recycled polycarbonate contains impurities - sometimes as little as $1 \%$. Thus the aim of this research is to find a way to improve the properties of recycled polycarbonate containing $1 \%$ or more of impurities. These impurities are likely to be Polyethylene (PE), Nylon, Polyethylene Terephthalate (PET), Acrylonitrile Butadiene Styrene (ABS), Polymethyl Methacrylate Acrylic (PMMA) and High Impact Polystyrene (HIPS). It has been found that some of the properties of polycarbonate undergo significant changes during the recycling process, while others do not change at all (see Table 3-1). The properties which change only slightly are the polymer tensile strength and tensile modulus; while those that decrease significantly are its impact strength, ductility, and transparency. The goal of this research is to attempt to restore the changeable properties to their original condition without affecting those which do not change. The aim is to produce a form of recycled polycarbonate that retains as many of the properties of virgin polycarbonate as possible. Transparency is the only property that cannot be improved, since recycled polycarbonate is made up of several colors. Virgin polycarbonate will lose its transparency even when only $0.5 \%$ of impurities are added. When the desired quality of recycled polycarbonate has been achieved, tests are conducted to ascertain the similarities between the properties of recycled polycarbonate and those found in the virgin material. These tensile, flexural, 
and impact tests were conducted on recycled, virgin, and on a blend of recycled and virgin materials to verify the similarities in the properties of the recycled polycarbonate compared to those found in the virgin material in the solid state. Rheological tests for shear viscosity, melt flow index, and dynamic mechanical analysis were also conducted in order to compare the performance of recycled polycarbonate to that of virgin polycarbonate in the melt state.

As indicated earlier, the level of impurities in one sample of recycled polycarbonate is less than $1 \%$. Two aspects of the influence of these impurities on properties of polycarbonate were examined: the percentage level and the types of impurities. Other thermoplastics (besides polycarbonate) found in electronics are ABS, HIPS, Nylon, LDPE, and PET. Five levels of impurities $(5 \%, 15 \%, 25 \%, 35 \%$, and 50\%) were added to unreinforced polycarbonate. Some mechanical and rheological tests were conducted on these samples. Glass fibers were also added to these five systems to see if this would improve the properties.

Tests were conducted to accumulate data and results to support the hypothesis that the addition of glass fiber to polycarbonate with impurities improves its properties, and that the properties of glass-reinforced polycarbonate with impurities are similar to those of glass reinforced virgin polycarbonate. If successful, this would means that the separation step in recycled thermoplastics is unnecessary, and that glass fibers can be successfully added to polycarbonate. The results obtained in this research were analyzed through the available theories such as the rules of mixture for short glass fibers. The mechanical and rheological theories discussed in Chapter two are used in Chapter 5 to model the experimental results obtained in this research. 
Table 3-1 Properties of recycled and virgin polycarbonate

\begin{tabular}{lcc}
\hline \hline Properties & $\begin{array}{c}\text { Virgin Polycarbonate } \\
\text { (Lexan HF1110) }\end{array}$ & $\begin{array}{c}\text { Recycled Polycarbonate } \\
\text { (unknown sources) }\end{array}$ \\
\hline Stress at break, psi & 9500 & 7500 \\
Modulus of elasticity, psi & 345000 & 338000 \\
Elongation at break, \% & 120 & 7 \\
Impact strength, ft-lb/in & 12 & 2 \\
Melt flow rate, gm/10min & 25 & 57 \\
\hline \hline
\end{tabular}

The process of improving the changed properties of recycled polycarbonate involves the addition of short glass fibers (see Figure 3-2). Although upon reinforcement, polycarbonate loses two of its outstanding properties - transparency and toughness - it gains good dimensional stability and superior creep (deformation under continuous load) resistance; it also gains excellent dielectric and flame retardant properties. 


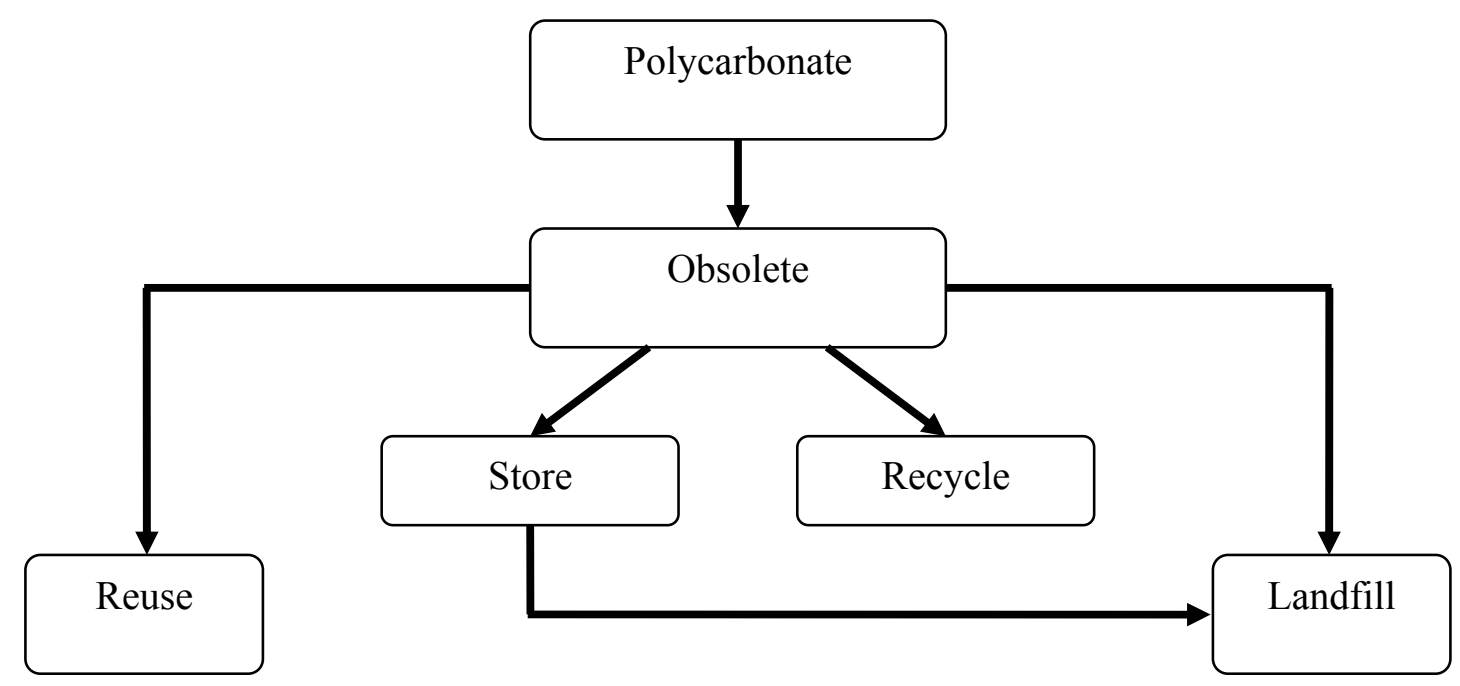

Figure 3-1 Life of polycarbonate in computer housing

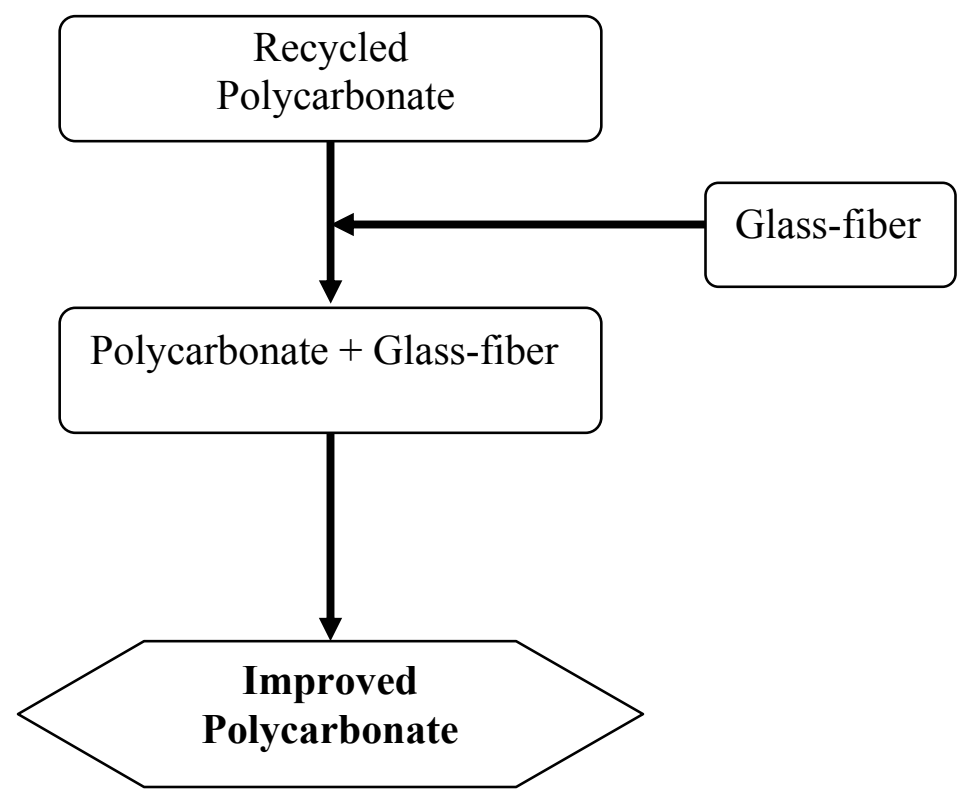

Figure 3-2 Method examined for improving the mechanical and rheological properties of recycled polycarbonate 


\section{Experimental Details}

\subsection{Materials}

The materials used in this research were virgin polycarbonate, polycarbonate with high glass fiber content, and recycled polycarbonate. The sources of these materials are indicated in Table 4-1. Detailed technical information about Lexan HF1110 and RTP307 is listed in Appendix A. The impurities that are added to polycarbonate are a mixture of LDPE, HIPS, PET, Nylon, and ABS. Fibers properties are listed in Appendix A.

Table 4-1 Brands and sources of materials used in this research.

\begin{tabular}{lll}
\hline \hline Material & Company & Trade Name \\
\hline Polycarbonate with 40\% Glass fiber & RTP & RTP 307 \\
Virgin Polycarbonate & GE Plastics & HF1110 \\
Recycled Polycarbonate & MBA polymers & ------- \\
\hline \hline
\end{tabular}

Short glass fibers are difficult and hazardous to handle. One way to facilitate ease of handling is to blend polycarbonate with high glass fiber content $(40 \%)$ with the recycled polymer and glass fibers. The mixture is then combined with recycled polymer until the desired glass fiber content is achieved. Three kinds of samples were prepared, each with the following compositions: $0,5,10,15$, and $20 \%$ by weight of glass fiber. The samples were: glass fiber with recycled polycarbonate, glass fiber with virgin polycarbonate, and glass fiber with a mixture of recycled and virgin polycarbonate (the mixture contained $50 \%$ recycled and $50 \%$ virgin polymer). The samples' contents are summarized in Figures (4-1)- (4-5). 


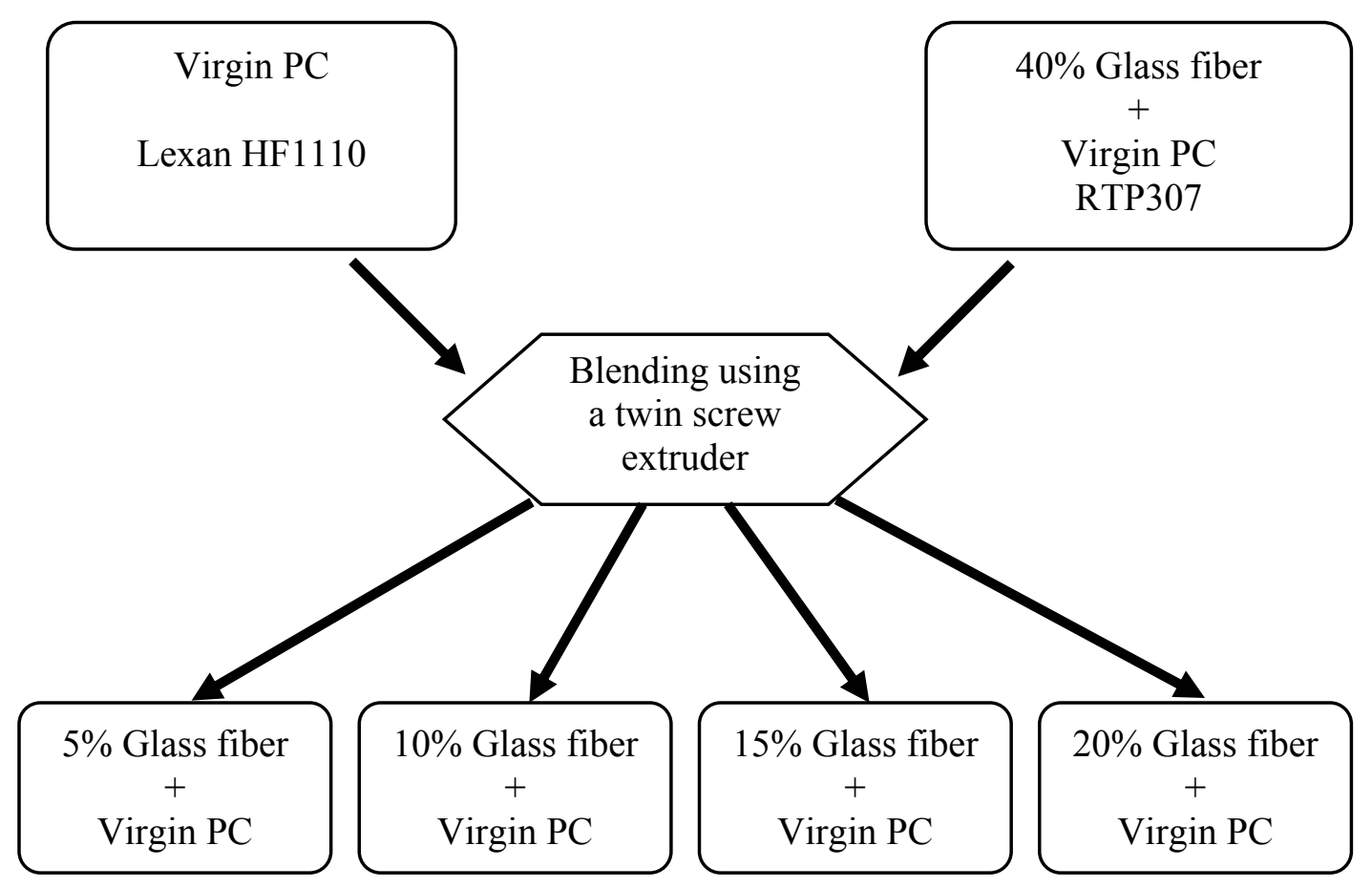

Figure 4-1 Schematic of blending virgin polycarbonate with glass fiber

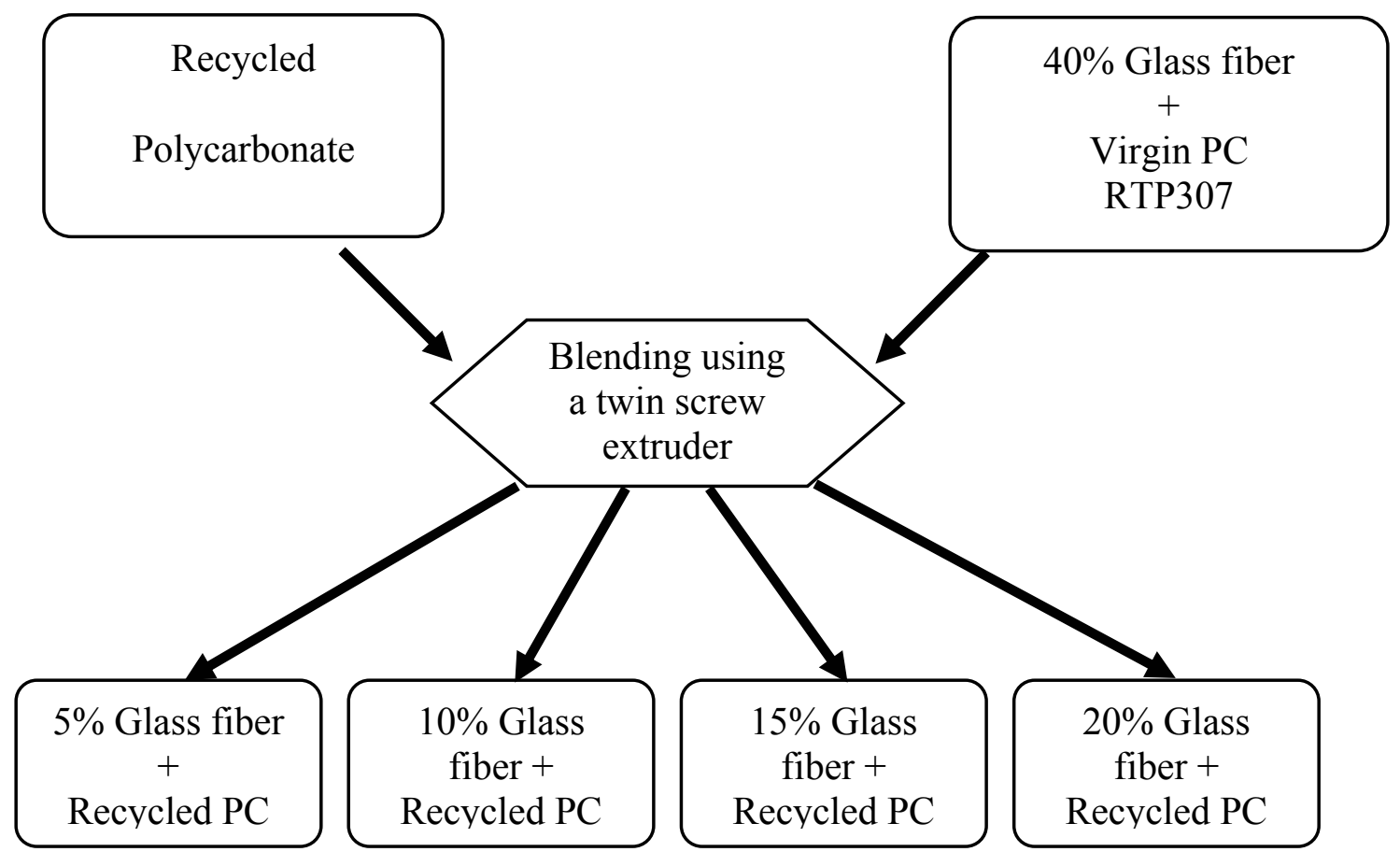

Figure 4-2 Schematic of blending recycled polycarbonate with glass fiber 


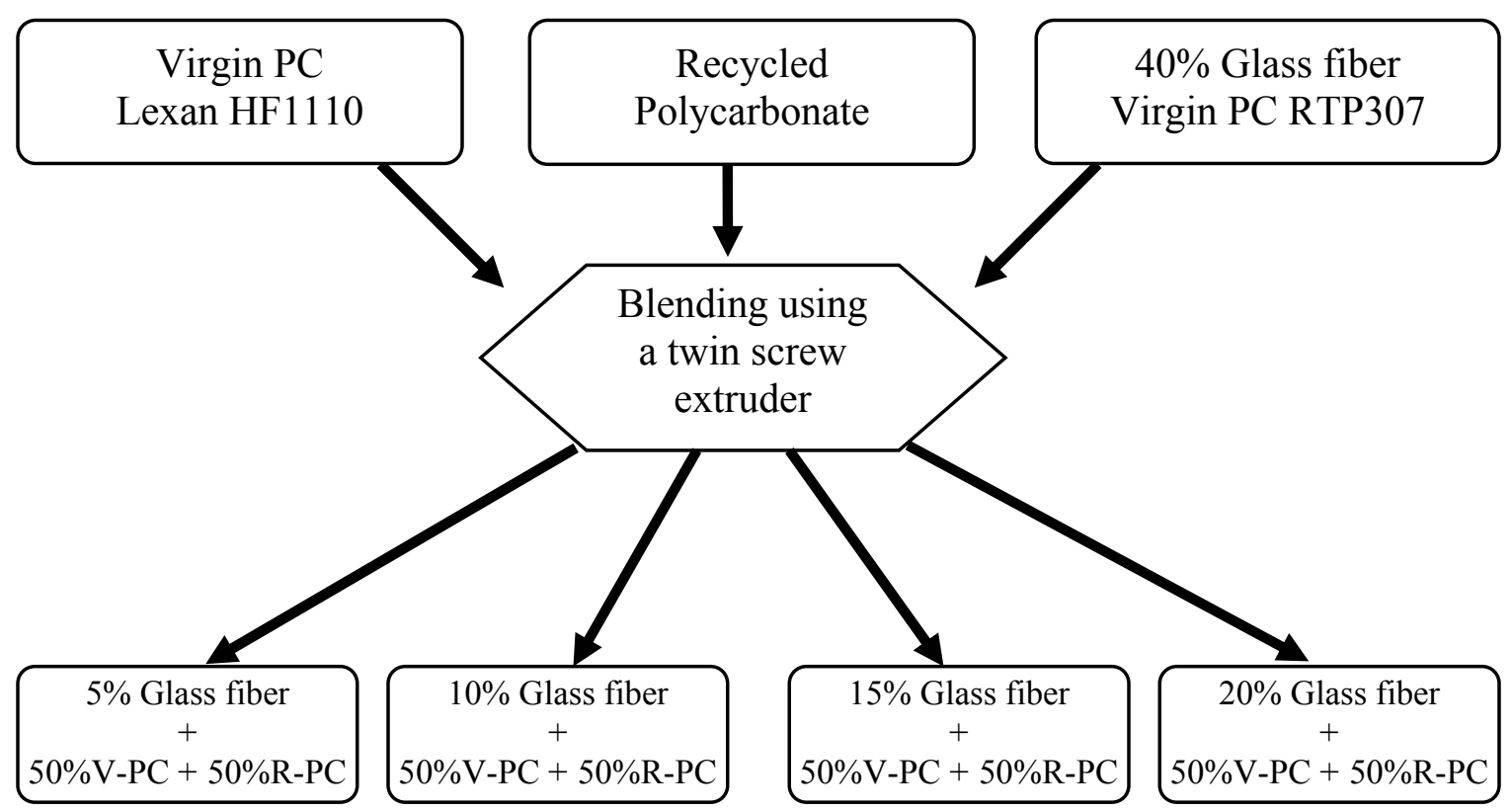

Figure 4-3 Schematic of blending glass fiber with polycarbonate $(50 \%$ Virgin $+50 \%$ recycle)

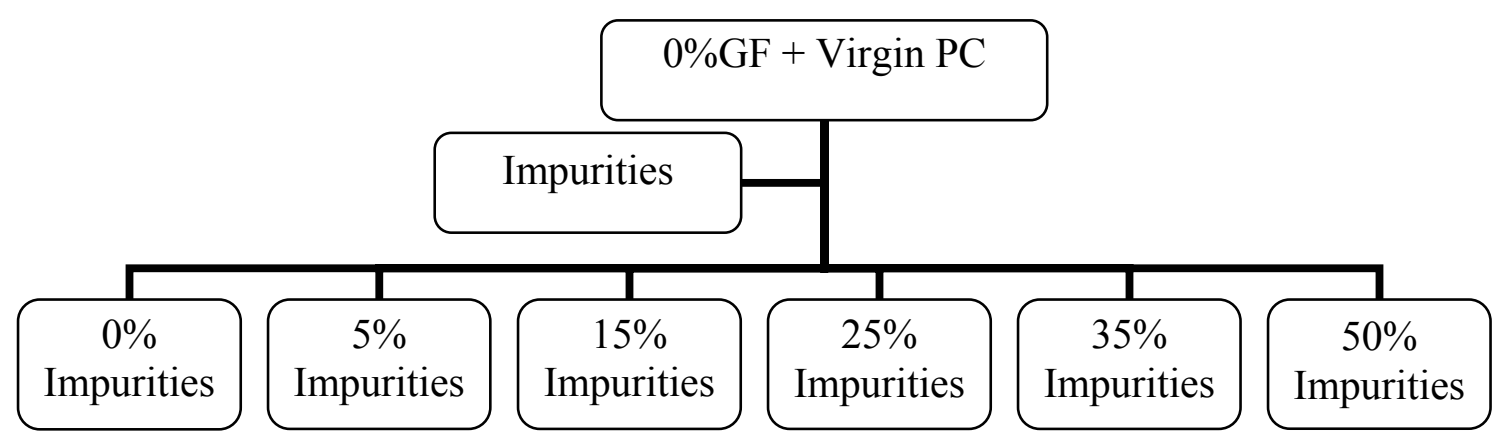

Figure 4-4 Schematic of blending polycarbonate and impurities

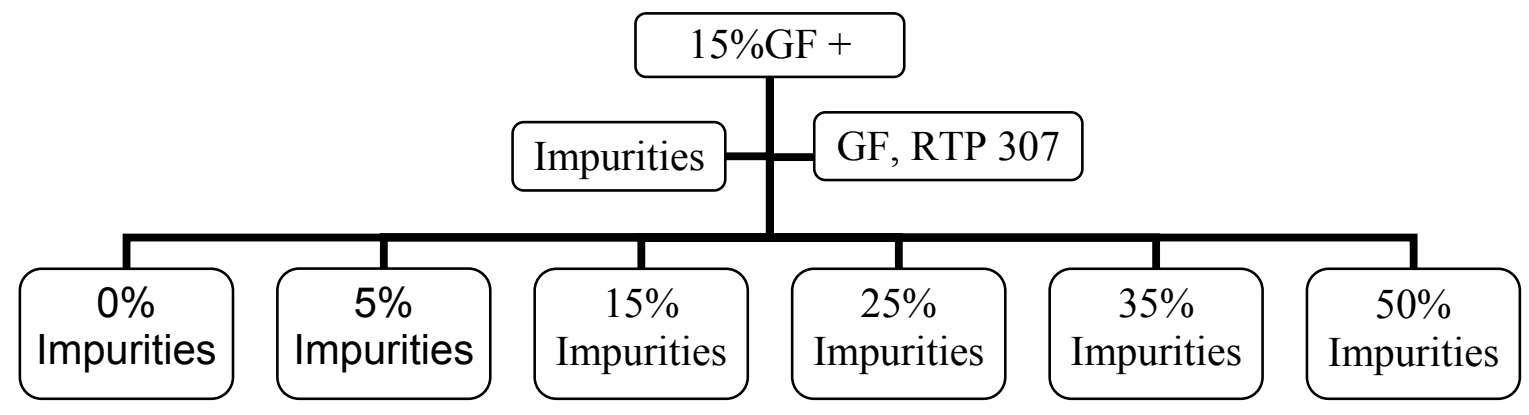

Figure 4-5 Schematic of blending glass fiber with polycarbonate and impurities 


\subsection{Sample preparation}

The following steps summarize the procedure for sample preparation:

- Desired amounts of materials are weighed.

- Materials are dried at a temperature of $120^{\circ} \mathrm{C}$ for at least 12 hours, but for no more than 24 hours (as recommended for polycarbonate).

- Pellets are placed in plastic zipper bags, and mixed by vigorous shaking.

- Materials are prepared by mechanical melt mixing in a Brabender twin-screw extruder. The extruder barrel has three heating zones, with a fourth heating zone being located between the barrel and the die (Figure 4-6). These conditions are chosen to ensure that no degradation occurs in the extrusion step.

$>\quad$ The temperature zones are $240,270,285,280^{\circ} \mathrm{C}$

$>\quad$ The screws rpm is 50

- Strands that exit the extruder are cooled using a water bath.

- The strands are pelletized with a pelletizer.

At this point, the blends are ready for compression molding, injection molding, measuring the melt flow indexer, measuring the molecular weight, and glass fibers size. 


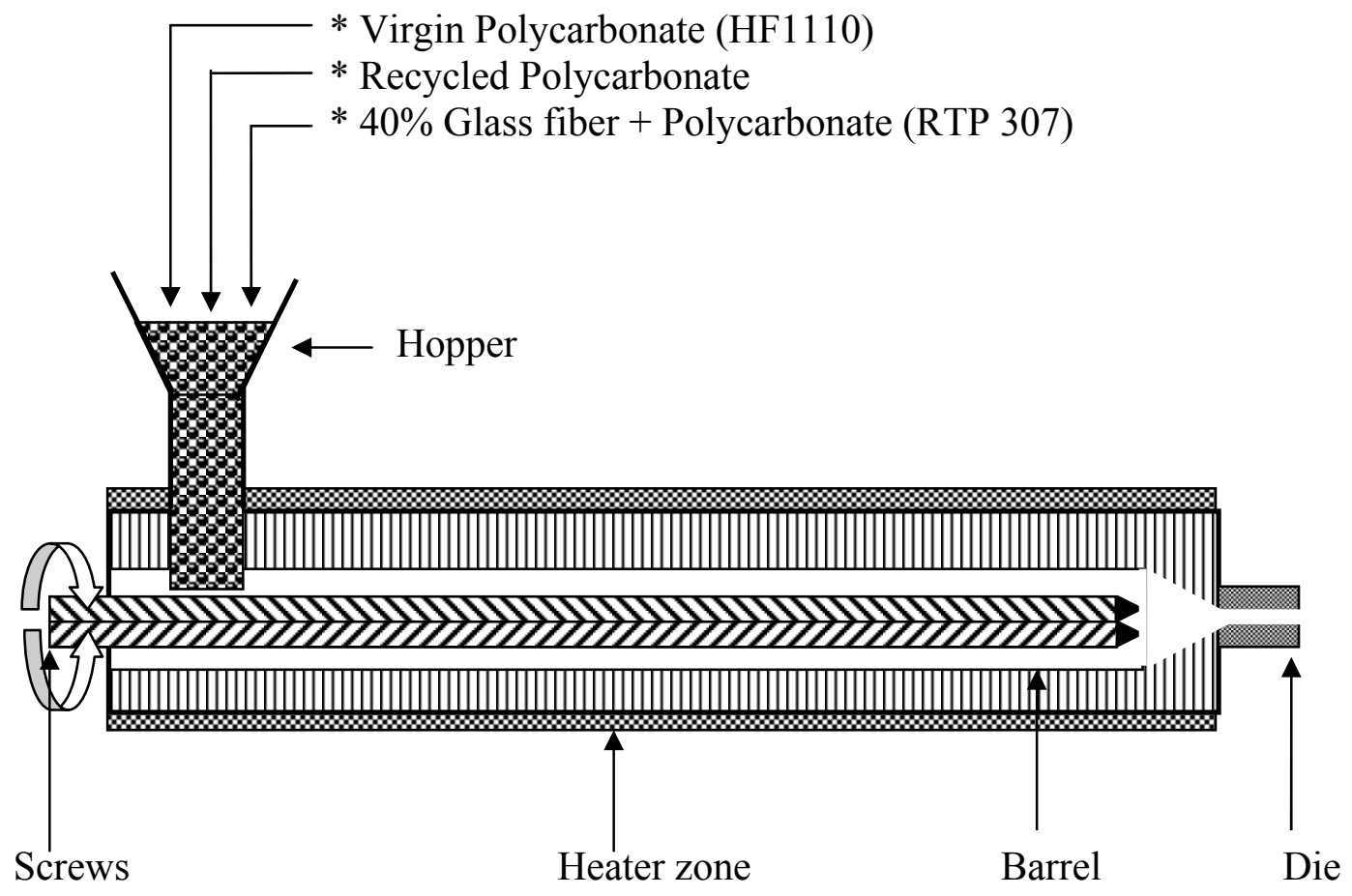

Figure 4-6 Schematic diagram for twin screw extruder

\subsection{Injection Molding}

The samples for the mechanical and some of the rheological tests were prepared using an injection molding machine. Pellets were dried for at least 12 hours in an oven at a temperature equal to $120{ }^{\circ} \mathrm{C}$. The following are the conditions under which the injection molding machine was operated:

- Zones temperatures $277,282,282,282{ }^{\circ} \mathrm{C}$

- Mold temperature $82{ }^{\circ} \mathrm{C}$

- Shot size $3.0 \mathrm{in}^{3}$

- Hold pressure 10,000 psi 
The mold produced samples for tensile, impact, flexural, and rheology tests (see Figure 4-

8).

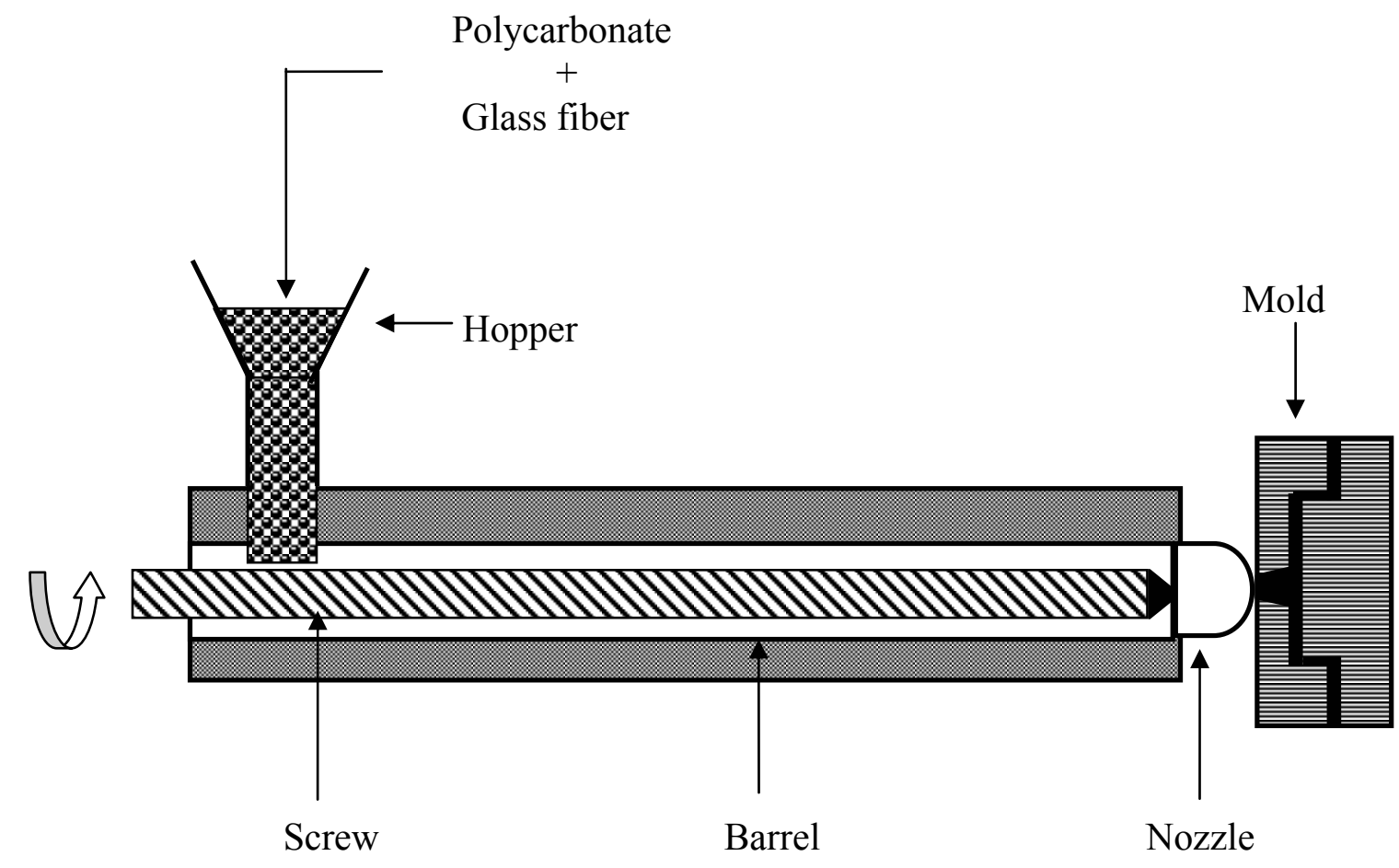

Figure 4-7 Schematic diagram of injection molding

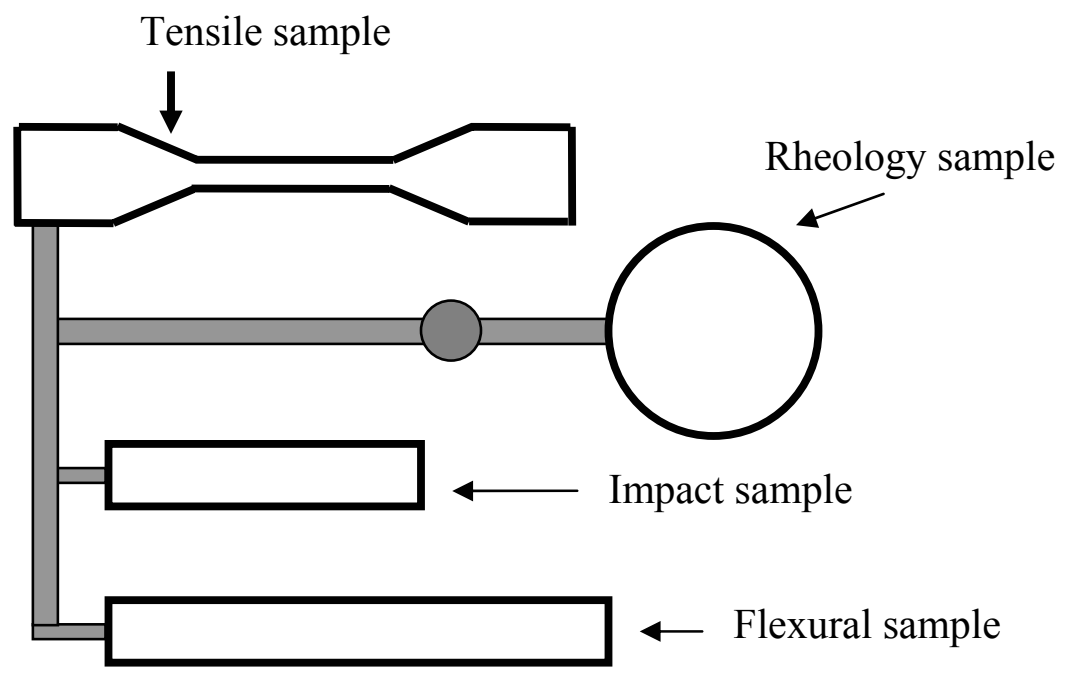

Figure 4-8 Injection molded samples 


\subsection{Percent glass fiber}

To ensure the correct fiber weight percentage after the extrusion step, the following procedure was followed:

- From each sample 30 grams were selected at random.

- The samples where burned in an oven for 5 hours at a temperature of $550^{\circ} \mathrm{C}$. The results are summarized in Table 4-2.

Table 4-2 Glass fiber content

\begin{tabular}{clc}
\hline $\begin{array}{c}\text { \% Glass fiber } \\
\text { expected }\end{array}$ & Polycarbonate & $\begin{array}{c}\text { \% Glass fiber } \\
\text { measured }\end{array}$ \\
\hline 5 & Virgin & 4.97 \\
10 & Virgin & 8.88 \\
15 & Virgin & 13.56 \\
20 & Virgin & 18.33 \\
$40^{*}$ & Virgin & 38.48 \\
5 & Recycled & 4.90 \\
10 & Recycled & 9.61 \\
15 & Recycled & 13.89 \\
20 & Recycled & 18.63 \\
5 & Virgin + Recycled & 4.74 \\
10 & Virgin + Recycled & 9.09 \\
15 & Virgin + Recycled & 14.07 \\
20 & Virgin + Recycled & 18.61 \\
\hline \hline
\end{tabular}

* RTP 307 
From the results in Table 4-2, it is concluded that the method of preparing the glass reinforced polycarbonate is valid. The measured glass fiber percentages are close to the expected percentages that were derived from calculations.

The experiments in this study can be divided into three parts (see Figures 4-9 and 4-10). First, mechanical tests were done to determine: tensile, flexural, impact, and hardness. Next the rheological tests examined shear viscosity, melt flow index and dynamic mechanical tests. The third set of tests measured the size, size distribution, and orientation of the fibers.

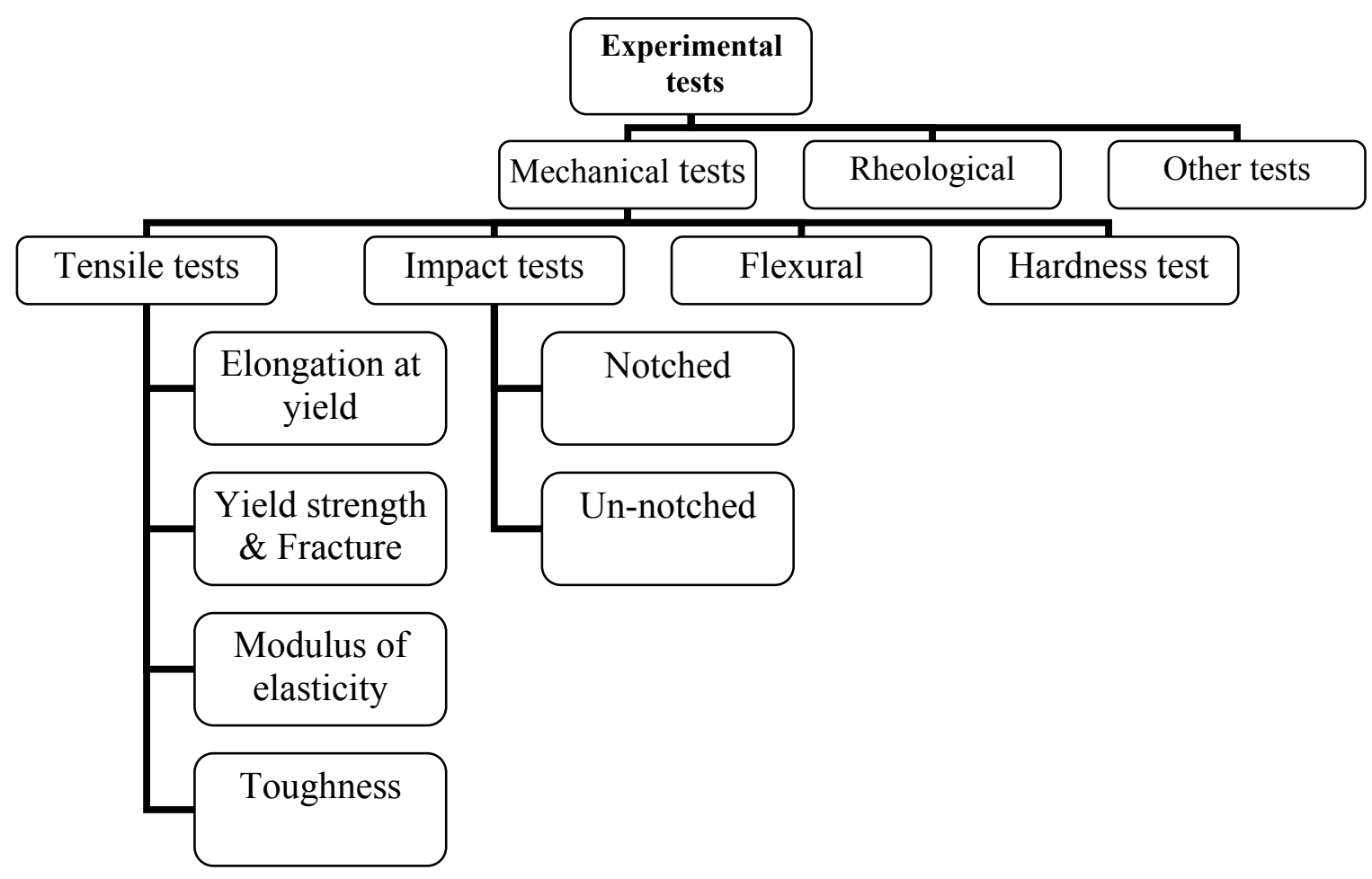

Figure 4-9 schematic of the experimental tests conducted 


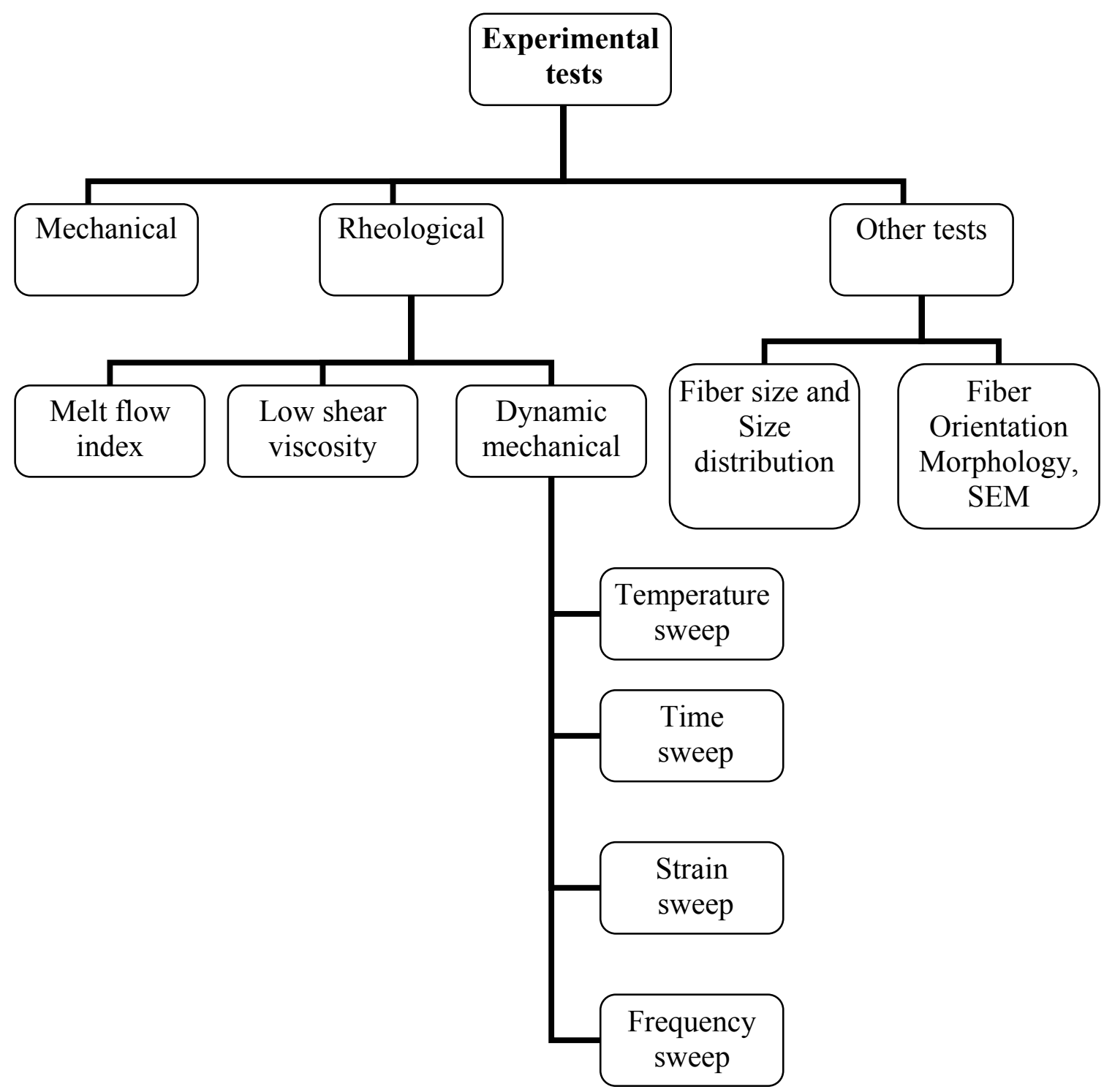

Figure 4-10 schematic of the experimental tests conducted 


\section{Mechanical Property Results}

The objective of the research is to study ways to improve the properties of the recycled polycarbonate and to examine the influence of impurities on un-reinforced and glass reinforced polycarbonate. As mentioned earlier the mechanical and rheological properties of recycled polycarbonate differ from the properties of the virgin polycarbonate. Several tests were conducted to measure the properties of recycled polycarbonate and polycarbonate with impurities and to compare these with properties of virgin polycarbonate. The tests were performed under the same conditions in order to achieve an accurate comparison of results for both recycled and virgin polycarbonate. Tensile, flexural, and impact strength tests were conducted to measure the mechanical properties. Melt flow index, dynamic mechanical and shear viscosity tests were conducted to measure the rheological properties.

\subsection{Fibers size and size distribution}

Measuring the glass fiber size and its size distribution helps us see the effect of the fiber's size on the mechanical properties, and to decide whether to change the method of introducing the fibers to the polymer matrix. Measuring the glass fiber size and distribution also allows us to compare the experimental results to the theories related to glass reinforced thermoplastics. The fibers that are present in the compound and in the molded component can have a very wide range of lengths and evenness of distribution. In order to obtain detailed information on the fiber length, considerable care must be exercised during the experiment. Fiber length distribution estimates are best obtained by means of a manual measurement of the lengths of a large number of fibers taken from a 
representative sample. This tedious procedure has been made considerably easier and more rapid with the introduction of image analysis equipment. Nevertheless, the crucial part of the exercise is sample preparation. It is necessary to recover the fibers by selective removal of the matrix so that they can be viewed when lying in a single plane. Removal of the matrix can be done using the following two techniques:

High temperature, a technique which involves temperatures well above the melting point of the matrix.

Chemical digestion using solvents or acids.

The choice of the technique depends upon the particular fiber/matrix combination. In this study, the first technique was used, although the other would have worked equally well. The fibers recovered for measurement are placed on a microscope slide. The fibers are photographed under a microscope. All fibers in each photograph are then measured for length. At least 500 should be measured for each of the 12 sample materials characterized. Fiber sizes and size distribution were measured for Polycarbonate RTP 307, which is the source of glass fiber. This gives the size of the fiber that has been added to the 12 samples (the initial glass fiber size). Finally, the fiber size after injection molding was measured for all 12 samples. The fiber size after the injection molding step is used to calculate the mechanical strength of all samples. Figure 5.1 shows typical short glass fibers after injection molding. Figure 5.2 shows the glass fiber distribution in virgin polycarbonate with $10 \%$ glass fiber. Table 5.1 summarizes the fiber length for all 12 samples and RTP 307 (polycarbonate with 40\% glass fiber). The average fiber length distribution information indicates that as the concentration of the glass fiber increases, the average fiber length distribution decreases. Statistical analysis of glass fiber length and 
glass fiber distribution for all materials is given in Appendix B.

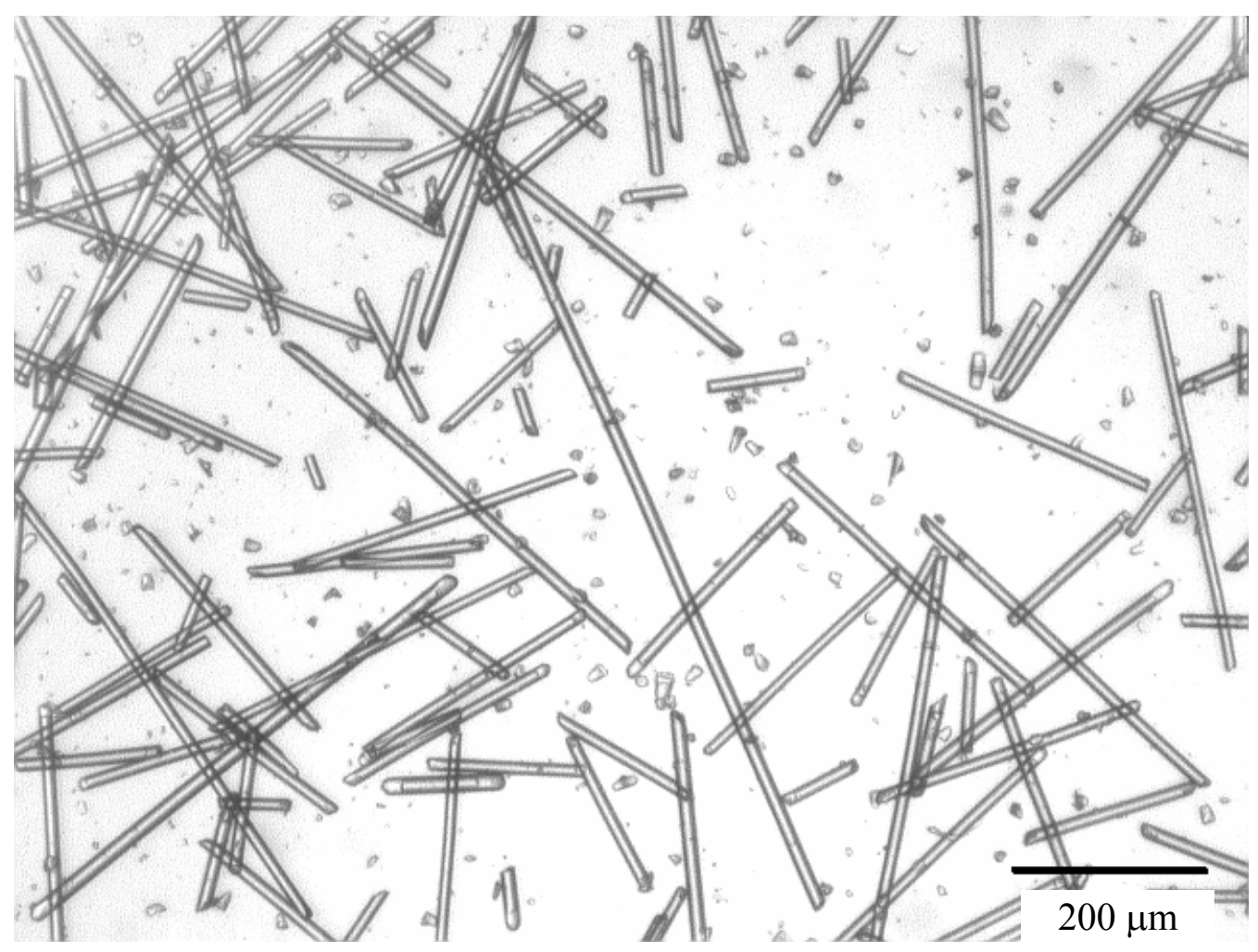

Figure 5-1 A dispersed field of short glass fibers recovered from injection molded $10 \%$ glass fiber and virgin polycarbonate

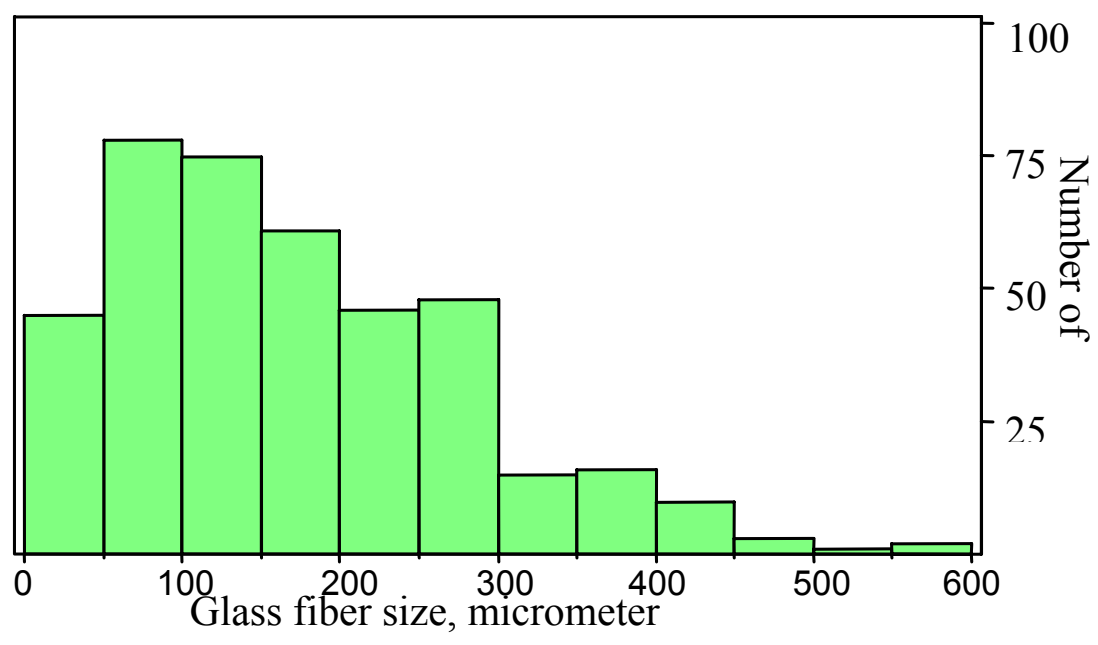

Figure 5-2 Fiber length distribution for 10\% GF and virgin polycarbonate 
Table 5-1 Average glass fiber length for three systems

\begin{tabular}{lccc}
\hline \%GF & $\begin{array}{c}\text { Virgin PC } \\
\mu \mathbf{m}\end{array}$ & $\begin{array}{c}\text { Recycled PC } \\
\mu \mathbf{m}\end{array}$ & $\begin{array}{c}\mathbf{5 0 \%} \mathbf{V}+\mathbf{5 0 \% R} \\
\boldsymbol{\mu m}\end{array}$ \\
\hline $\mathbf{5}$ & 176 & 176 & 173 \\
$\mathbf{1 0}$ & 172 & 171 & 172 \\
$\mathbf{1 5}$ & 161 & 163 & 159 \\
$\mathbf{2 0}$ & 156 & 155 & 155 \\
$\mathbf{2}$ & & & \\
\hline
\end{tabular}

\subsection{Mechanical behavior of the polycarbonate system}

Characterization of the mechanical behavior of the polycarbonate system includes the measurement of tensile strength, elastic modulus, percent elongation (at yield and break), flexural strength, and impact strength. The rules of mixture of glass reinforced thermoplastics can be used to analyze the experimental results.

\subsubsection{Tensile strength}

The tests for tensile strength and modulus were conducted using a universal Instron machine (see Figure 5-4). The American Society for Testing and Materials (ASTM D638) guideline was followed while conducting the tensile strength tests. According to ASTM D638 the rate of stretching of un-reinforced polycarbonate is 2 inches/minute, whereas for reinforced polycarbonate the rate is 0.2 inches/minute. In measuring the modulus of elasticity, an Extensometer was used (as recommended by ASTM). Sample dimensions are: 


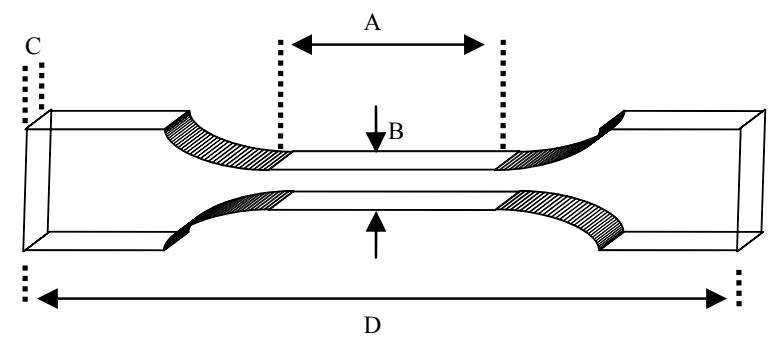

A: 2.5 inch, B: 0.5 inch, C: 0.125 inch, D: 6.5 inch

Figure 5-3 Tensile bar dimensions Type I

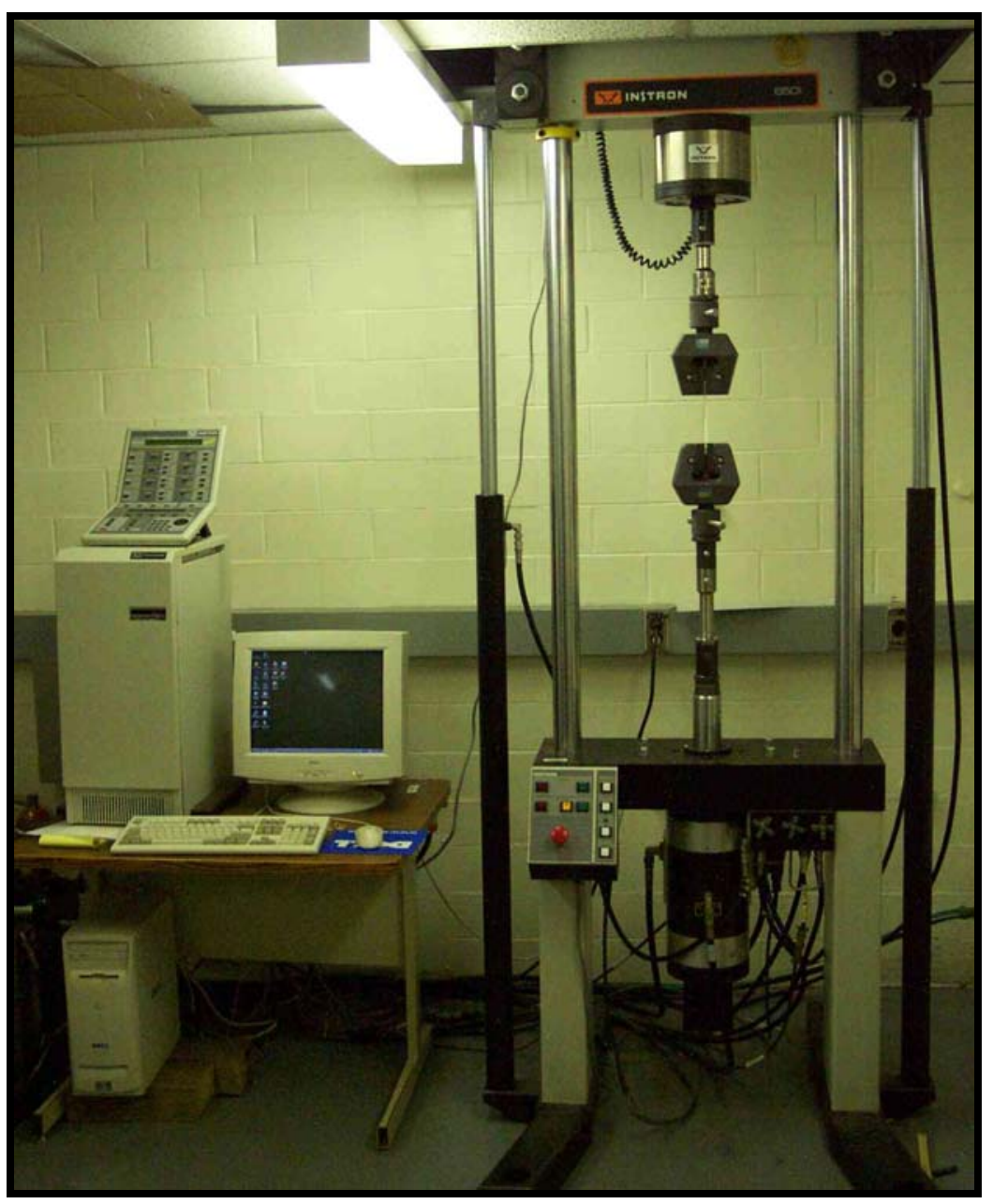

Figure 5-4 Instron machine used to measure the tensile and flexural strength 
Results of the measurements are shown in Figures 5-5 through 5-9. They show that the addition of glass fiber can significantly increase the tensile strength (both yield and fracture strengths). This trend is well documented in the literature on virgin polycarbonate. We see the same trend for recycled polycarbonate and for the blend of virgin and recycled polycarbonate. It is significant that the yield strength of recycled material with $0 \%$ glass fiber is higher than the yield strength of virgin material, while the yield strength of the blend is somewhere in between. The recycled material is more brittle and is more resistant to deformation than the virgin material.

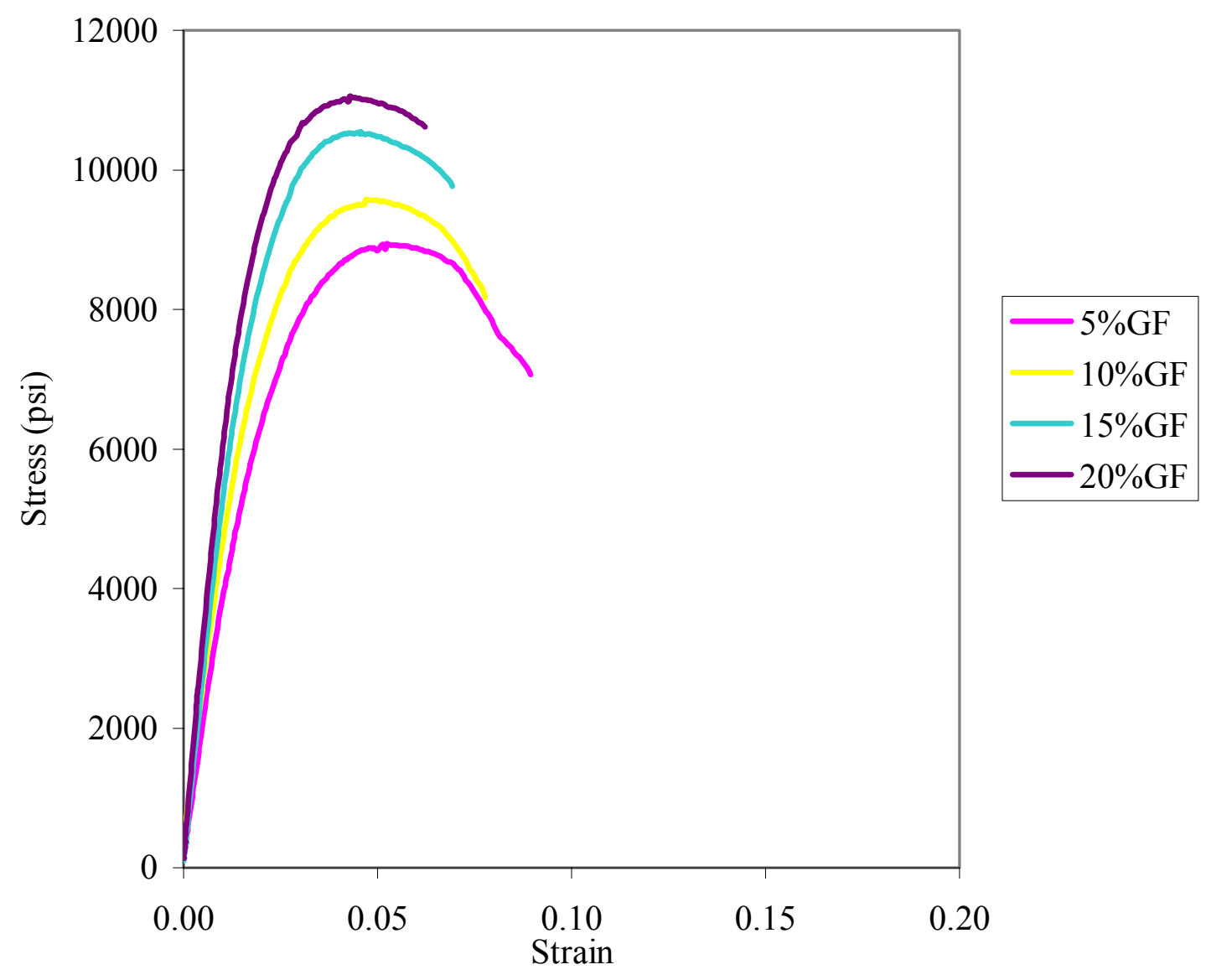

Figure 5-5 Stress strain curve for virgin PC containing glass fiber 


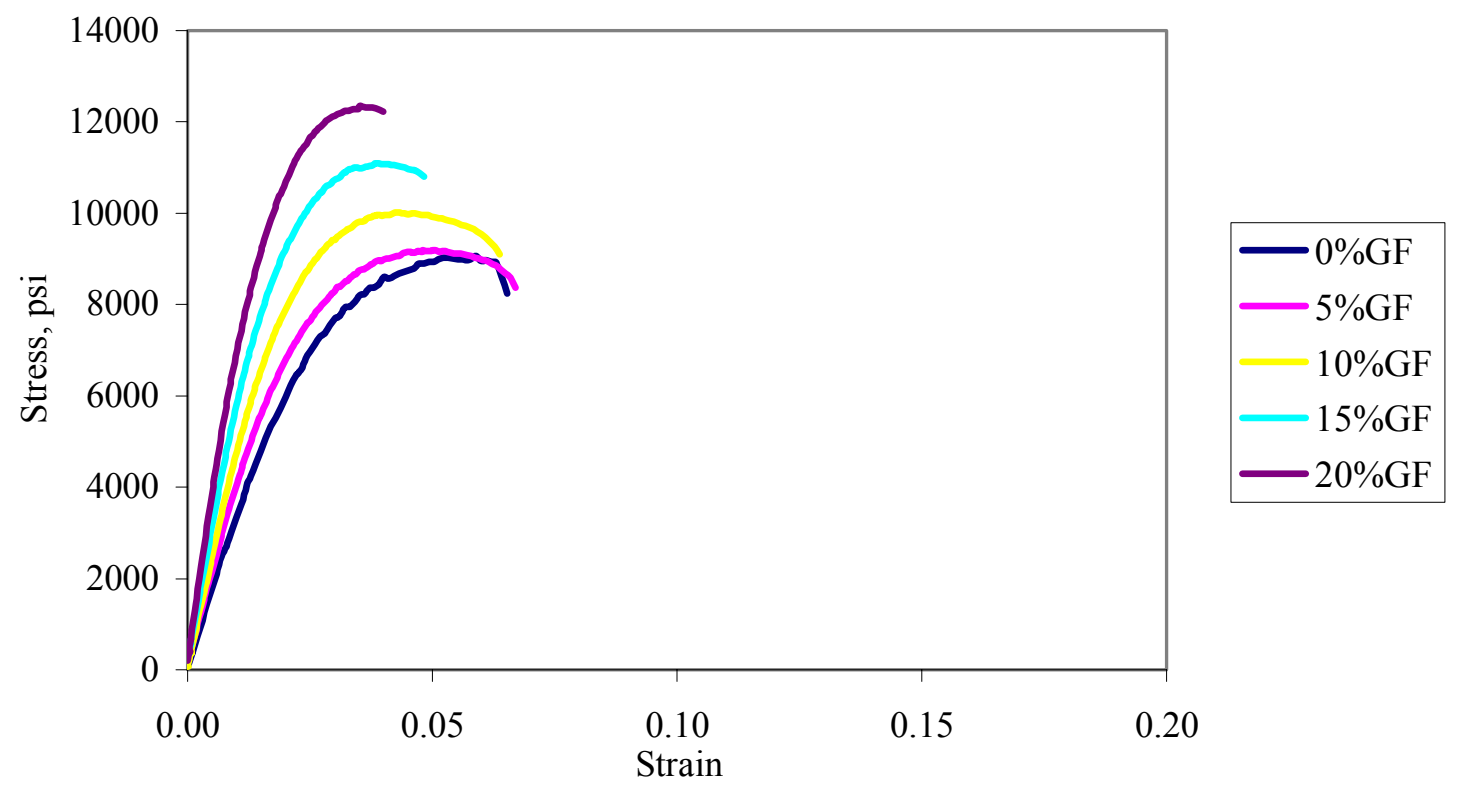

Figure 5-6 Stress-strain curve for un-reinforced and glass reinforced recycled PC

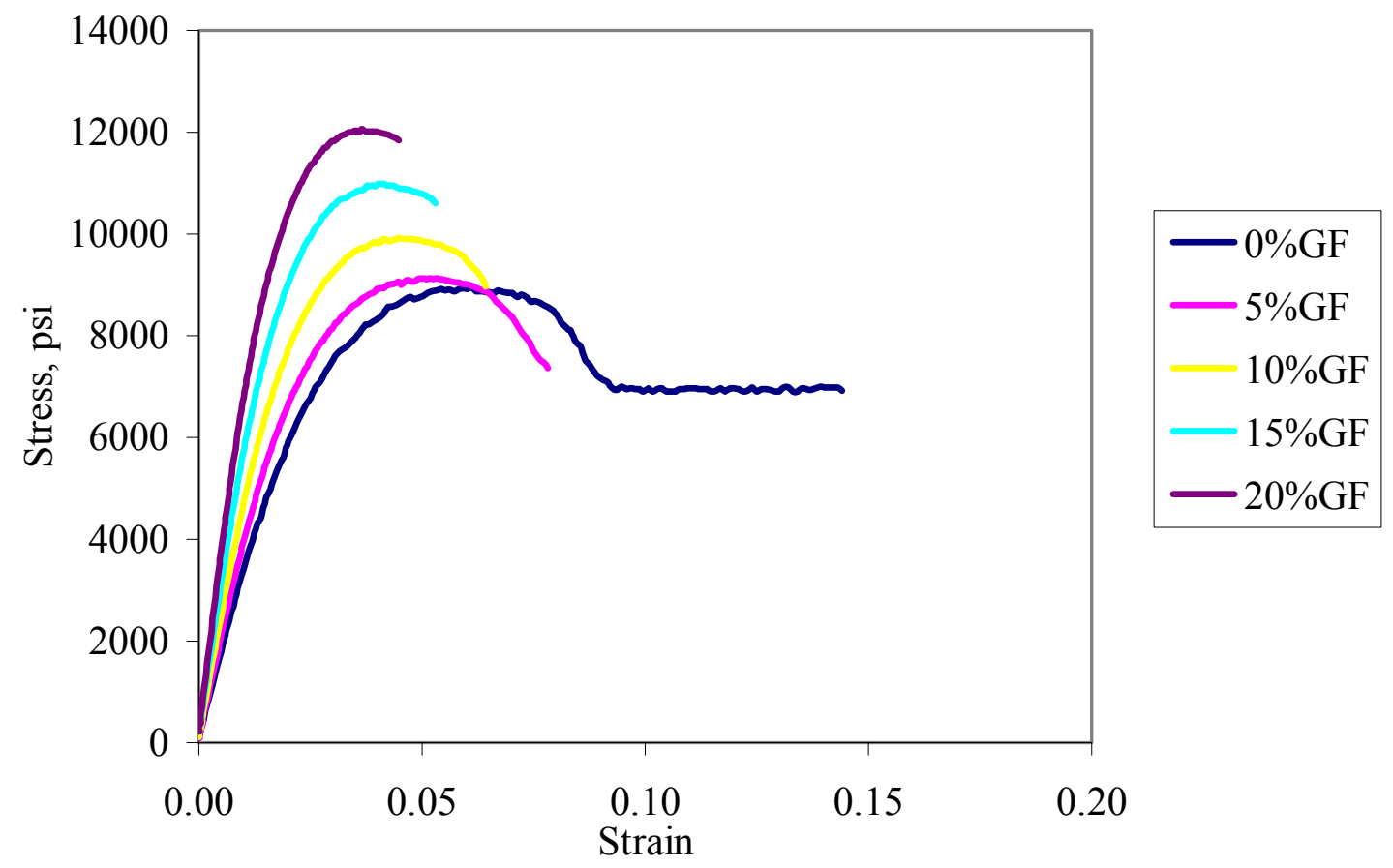

Figure 5-7 Stress-strain curve for un-reinforced and glass reinforced 50\% recycled PC+ $50 \%$ virgin $\mathrm{PC}$ 


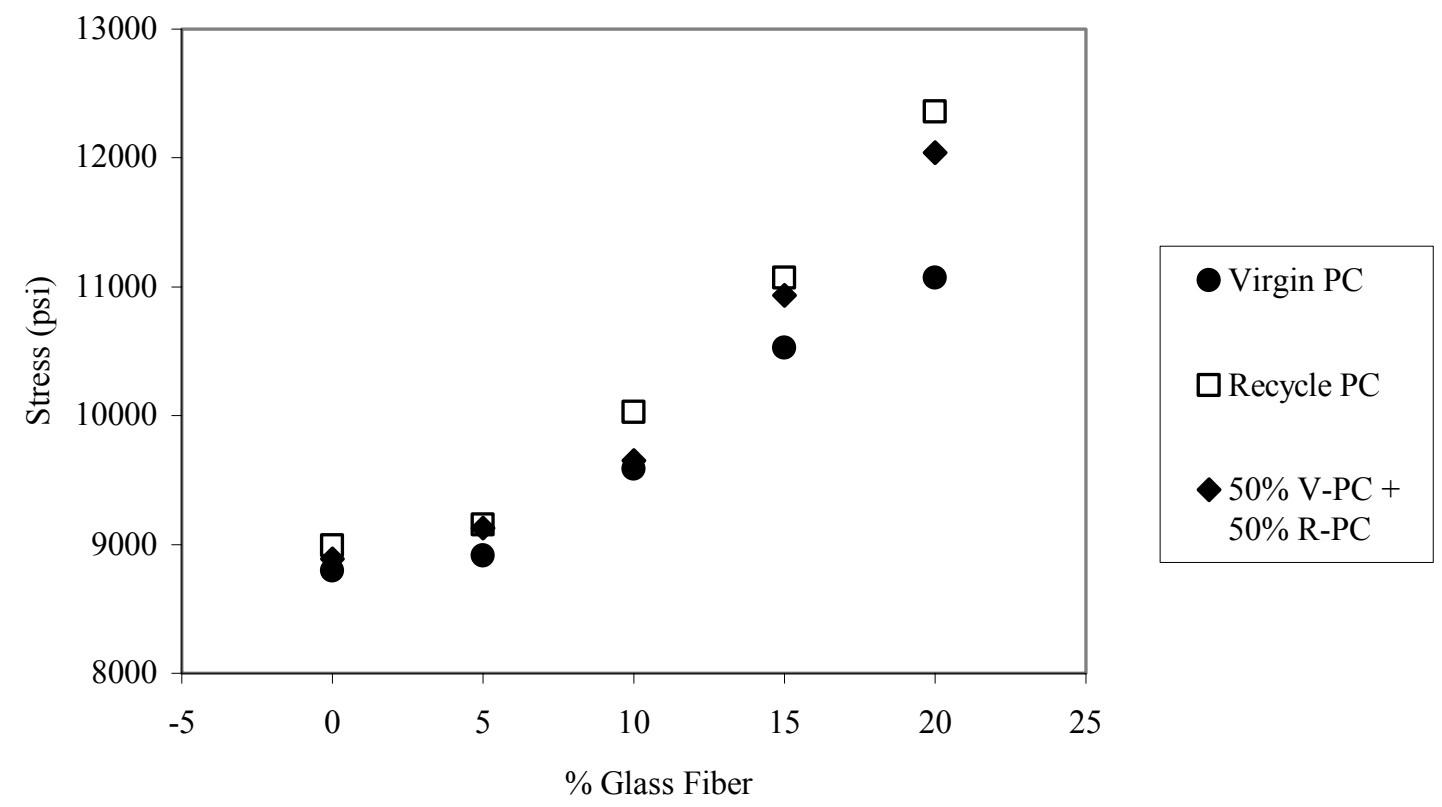

Figure 5-8 Effect of glass fiber on yield strength of polycarbonate

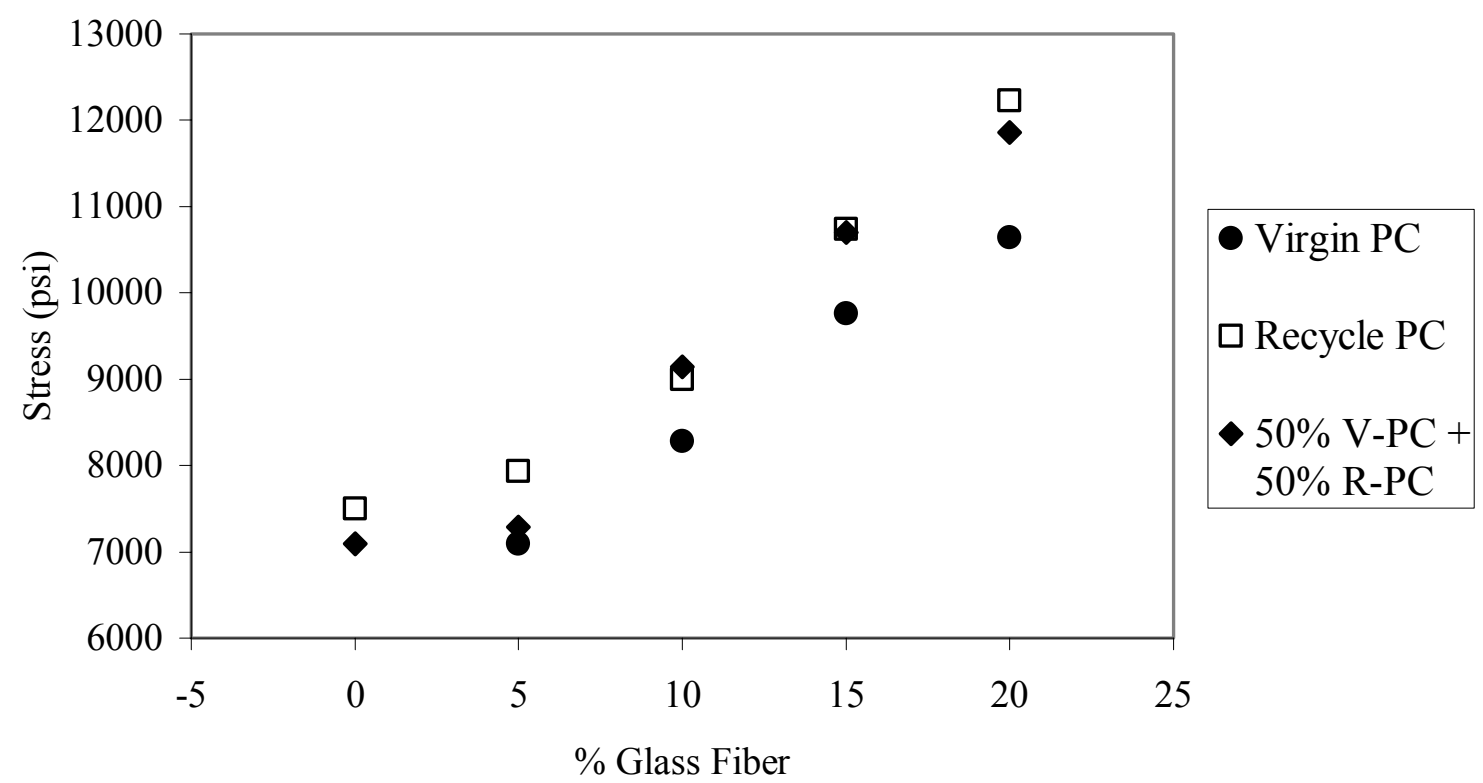

Figure 5-9 Effect of glass fiber on strength at fracture of polycarbonate 
It was pointed out earlier that one of the objectives of this study was to explore the simplest theory for the prediction of composite stiffness and strength in short fiberreinforced thermoplastics. For this purpose, the distribution of fiber orientation was determined first. As can be seen, the ultimate strength of the polymer matrix is enhanced by the addition of glass fibers. As illustrated in Figure 5-10, the tensile strength varies essentially, linearly with volume fraction $\left(\phi_{\mathrm{f}}\right)$. The linearity of the tensile strength with $\phi_{\mathrm{f}}$ indicates that the tensile strength should conform to the rule of mixtures.

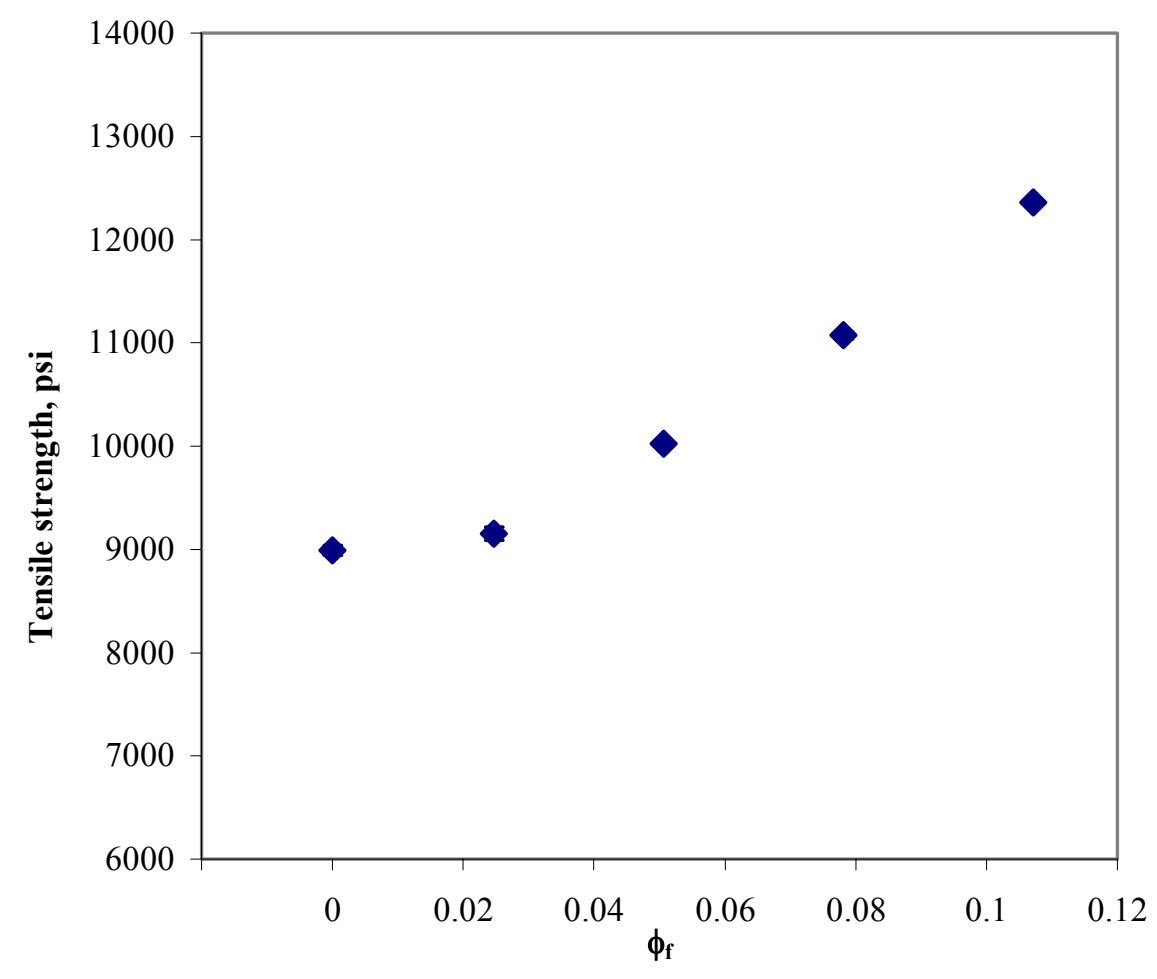

Figure 5-10 The tensile strength versus volume fraction of glass fibers for recycled PC

According to Kelly and Tyson ${ }^{62}$ the ultimate strength of the short fiber composites, $\sigma_{\mathrm{c}}$, may be related to $\phi_{\mathrm{f}}$ according to the following rule of mixtures; 


$$
\sigma_{c}=\sigma_{f} \phi_{f} \eta_{L} \eta_{o}+\sigma_{m}\left(1-\phi_{f}\right)
$$

$\sigma_{\mathrm{c}}$ : Composite ultimate strength

$\sigma_{\mathrm{f}}$ : Fiber strength

$\sigma_{\mathrm{m}}$ : Matrix strength

$\phi_{f}:$ Fiber volume fraction which is;

$$
\phi_{\mathrm{f}}=\left[1+\frac{\rho_{\mathrm{f}}}{\rho_{\mathrm{m}}}\left(\frac{1}{\mathrm{~W}_{\mathrm{f}}}-1\right)\right]^{-1}
$$

$\rho_{f}$ : Density of the fiber

$\rho_{\mathrm{m}}$ : Density of the matrix

$\mathrm{W}_{\mathrm{f}}$ : Weight of the fiber

$\eta_{\mathrm{o}}$ : Orientation efficiency of the reinforcing fibers (having value of 1 for alignedlongitudinal, 0 for aligned-transverse)

$\eta_{\mathrm{L}}$ : Reinforcing effectiveness of the short fibers. For the case in which the average length, $\mathrm{L}_{\mathrm{f}}$ is less than the critical value, $\mathrm{L}_{\mathrm{c}}$, is given by ${ }^{61}$

$$
\eta_{\mathrm{L}}=\frac{\mathrm{L}_{\mathrm{f}}}{2 \mathrm{~L}_{\mathrm{c}}}
$$

and for $\mathrm{L}_{\mathrm{f}}>\mathrm{L}_{\mathrm{c}}$ it is given by ${ }^{61}$

$$
\eta_{\mathrm{L}}=1-\frac{\mathrm{L}_{\mathrm{f}}}{2 \mathrm{~L}_{\mathrm{c}}}
$$

where Lc, can be calculated from the following equation;

$$
\mathrm{L}_{\mathrm{c}}=\frac{\mathrm{d} \sigma_{\mathrm{f}}}{2 \tau_{\mathrm{m}}}
$$


The shear strength of the matrix, $\tau_{\mathrm{m}}$, is taken to be half its tensile yield strength. From the experimental data we see that the influence of glass fiber concentration on the tensile strength follows a straight line. One can rearrange equation (5-1)

$$
\sigma_{c}=\sigma_{m}+\phi_{f} \sigma_{m}\left[\frac{L_{f} \eta_{o} \sigma_{f}}{2 L_{c} \sigma_{m}}-1\right]
$$

The Slope of the line equals $\sigma_{\mathrm{m}}\left[\frac{\mathrm{L}_{\mathrm{f}} \eta_{\mathrm{o}} \sigma_{\mathrm{f}}}{2 \mathrm{~L}_{\mathrm{c}} \sigma_{\mathrm{m}}}-1\right]$ and from the slope, one can calculate $\eta_{\mathrm{o}}$

Table 5-2 summarizes the orientation and length efficiency for virgin, recycled, and blend of the virgin and recycled glass-reinforced polycarbonate. Detailed calculations are given in Appendix C.

Figure 5-11 shows that the influence of impurities on the strength of polycarbonate is minimal. Thus, a thermoplastics blend that contains no more than $25 \%$ of impurities will maintain the desired level of tensile strength. This finding suggests that we can eliminate the need for the separation step in recycling thermoplastics. 
Table 5-2 Reinforcement efficiencies for glass reinforced polycarbonate

\begin{tabular}{|c|c|c|c|c|}
\hline \multicolumn{5}{|c|}{ Virgin Polycarbonate } \\
\hline$\% w t$ & $\% \phi_{\mathrm{f}}$ & $\mathbf{L}_{\mathrm{f},}, \mu \mathrm{m}$ & $\eta_{\mathrm{L}}$ & $\eta_{\mathrm{o}}$ \\
\hline 5 & 2.5 & 174 & 0.26 & 0.50 \\
\hline 10 & 5.1 & 172 & 0.25 & 0.51 \\
\hline 15 & 7.8 & 161 & 0.24 & 0.54 \\
\hline 20 & 10.7 & 156 & 0.23 & 0.56 \\
\hline \multicolumn{5}{|c|}{ Recycled Polycarbonate } \\
\hline wt & $\phi_{\mathrm{f}}$ & $\mathrm{L}_{\mathrm{f},} \mu \mathrm{m}$ & $\eta_{\mathrm{L}}$ & $\eta_{\mathrm{o}}$ \\
\hline 5 & 2.5 & 176 & 0.26 & 0.63 \\
\hline 10 & 5.1 & 171 & 0.26 & 0.65 \\
\hline 15 & 7.8 & 163 & 0.24 & 0.68 \\
\hline 20 & 10.7 & 155 & 0.23 & 0.72 \\
\hline \multicolumn{5}{|c|}{$50 \%$ Virgin $+50 \%$ Recycled Polycarbonate } \\
\hline wt & $\phi_{\mathrm{f}}$ & $\mathbf{L}_{\mathrm{f}, \mu \mathrm{m}}$ & $\eta_{\mathbf{L}}$ & $\eta_{\mathrm{o}}$ \\
\hline 5 & 2.5 & 173 & 0.26 & 0.62 \\
\hline 10 & 5.1 & 172 & 0.25 & 0.62 \\
\hline 15 & 7.8 & 159 & 0.24 & 0.67 \\
\hline 20 & 10.7 & 155 & 0.23 & 0.69 \\
\hline
\end{tabular}




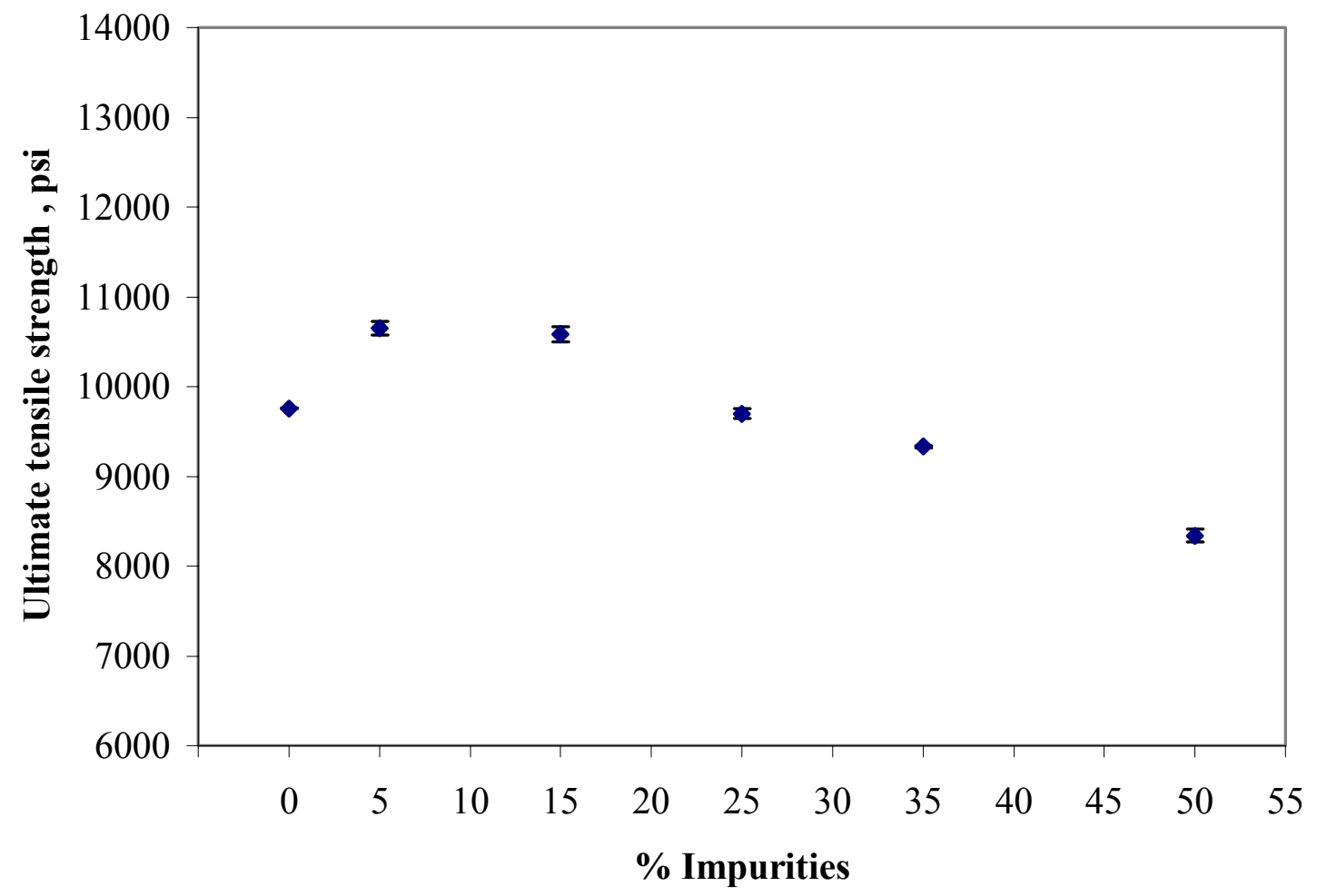

Figure 5-11 Effect of impurities on 15\% glass reinforced polycarbonate (impurities are ABS, HIPS, PET, LDPE, and Nylon)

To predict the tensile strength of glass-reinforced polycarbonate with impurities, the orientation efficiency of $15 \%$ glass-reinforced virgin polycarbonate was used with the rules of mixtures (see Figure 5-12). The tensile strength of unreinforced polycarbonate with impurities, $\sigma_{\mathrm{m}}$, was measured and equation (5-6) was used to predict the tensile strength of glass reinforced polycarbonate with impurities (see Table 5-3). Based on Figure 5-12, it is clear that the modified rule of mixture can be used to predict the tensile strength of glass reinforced polycarbonate with impurities. 
Table 5-3 15\% glass reinforced polycarbonate with impurities prediction of tensile strength

\begin{tabular}{cccccc}
\hline \hline \% Impurities & $\sigma_{\mathbf{m}}$ & $\mathbf{L}_{\mathbf{c}}$ & $\eta_{\mathbf{L}}$ & $\eta_{\mathbf{o}}$ & $\sigma_{\mathbf{c}}$ predicted \\
& $\mathbf{p s i}$ & $\mu \mathbf{m}$ & & $\mathbf{p s i}$ \\
\hline 5 & 8816 & 340 & 0.24 & 0.54 & 10627 \\
15 & 8551 & 351 & 0.23 & 0.54 & 10308 \\
25 & 7915 & 379 & 0.21 & 0.54 & 9541 \\
35 & 7174 & 418 & 0.19 & 0.54 & 8648 \\
\hline \hline
\end{tabular}

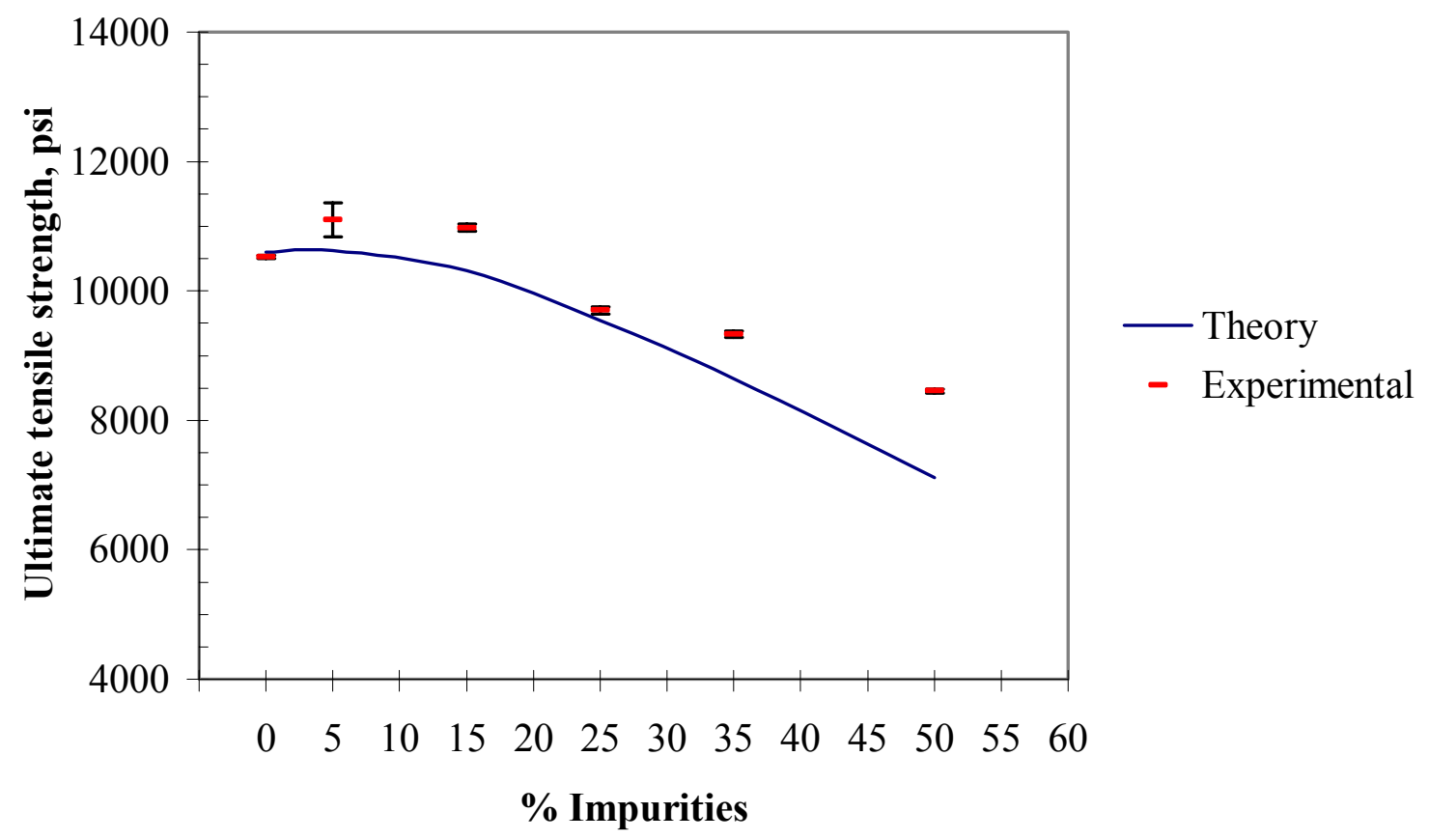

Figure 5-12 Using rules of mixture to predict the tensile strength of $15 \%$ glass reinforced polycarbonate containing impurities 


\subsubsection{Modulus of elasticity}

Characterization of the mechanical behavior of the glass-reinforced polycarbonate system includes a measurement of the elastic modulus from the stress-strain data. Table

5-4 summarizes the initial elastic modulus of the three systems of polycarbonate. Results indicate that the modulus of the unfilled polymer is enhanced by the addition of glass fiber. The modulus of elasticity increases as the glass fiber content increases for all three blends as seen in Figure 5-13.

Table 5-4 Summary of the elastic modulus

\begin{tabular}{cccc}
\hline \hline & & \multicolumn{3}{c}{ Modulus of elasticity, psi } \\
\% Glass Fiber & Virgin PC & Recycled PC & $\mathbf{5 0 \%}$ Virgin + 50\% Recycled \\
\hline \hline 0 & 302938 & 338237 & 310945 \\
5 & 373660 & 407607 & 387494 \\
10 & 448001 & 480589 & 475218 \\
15 & 543597 & 602599 & 580264 \\
\hline
\end{tabular}




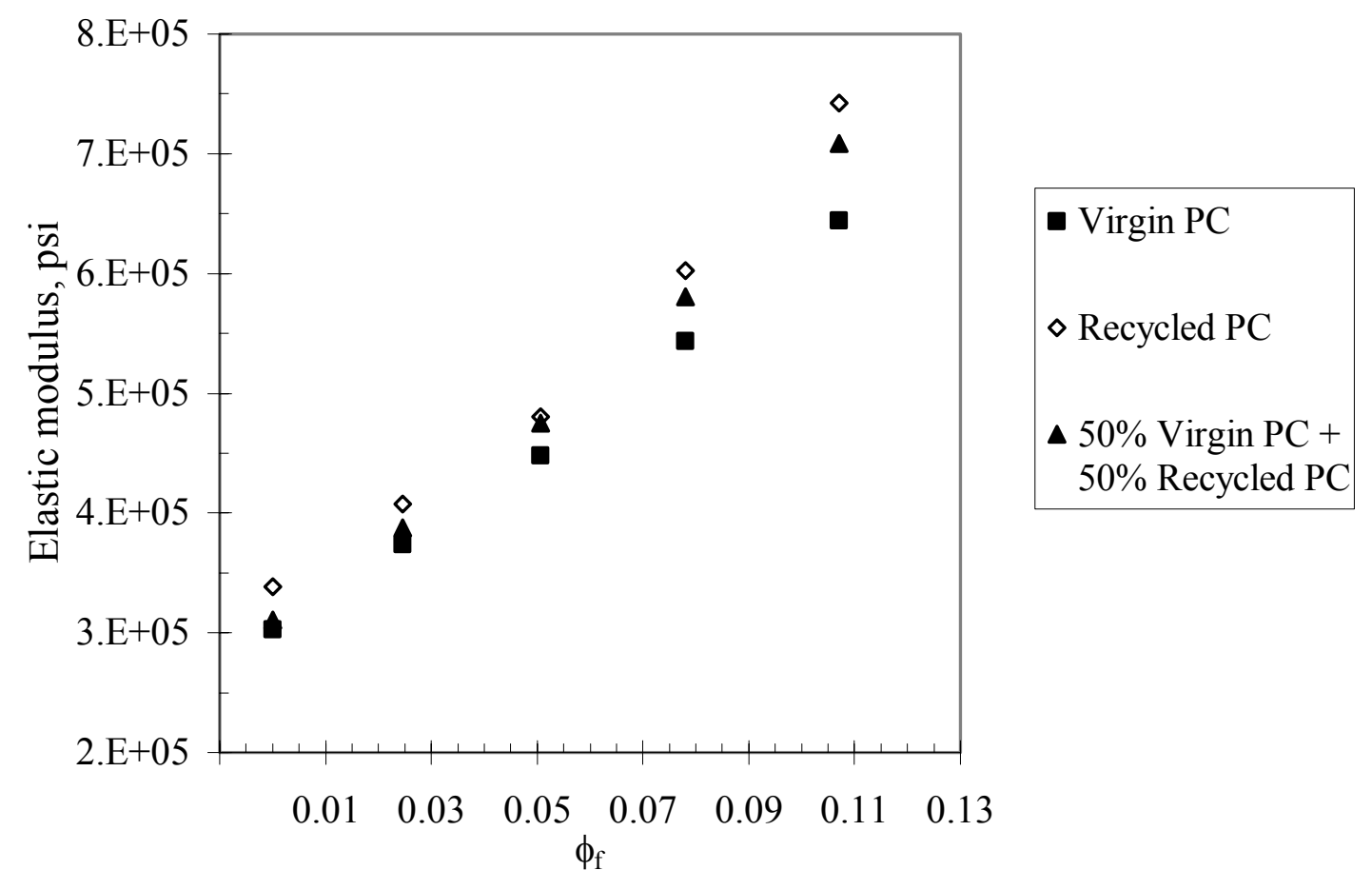

Figure 5-13 Effect of glass fiber on the modulus of elasticity

It is also evident from Figure 5-13, that the variation of the elastic modulus with respect to $\phi_{\mathrm{f}}$ is reasonably linear and this suggests that the behavior follows some form of the rule of mixtures. The form of rules of mixture is in the form ${ }^{63}$;

$$
\mathrm{E}_{\mathrm{c}}=\mathrm{E}_{\mathrm{f}} \phi_{\mathrm{f}} \eta_{\mathrm{L}} \eta_{\mathrm{o}}+\mathrm{E}_{\mathrm{m}}\left(1-\phi_{\mathrm{f}}\right)
$$

$\mathrm{E}_{\mathrm{c}}$ : Composite modulus

Ef: Fiber modulus 10500000 psi

$\mathrm{E}_{\mathrm{m}}$ : Matrix modulus

$\phi_{f}:$ Fiber volume fraction

$\eta_{0}$ : Orientation efficiency of the reinforcing fibers (having value of 1 for alignedlongitudinal, 0 for aligned-transverse) 
$\eta_{L}$ : Reinforcing effectiveness of the short fibers which can be estimated from

$$
\begin{aligned}
& \eta_{\mathrm{L}}=1-\frac{\tanh \mathrm{x}}{\mathrm{x}} \\
& \mathrm{x}=\frac{\mathrm{L}_{\mathrm{f}}}{2}\left(\frac{8 \mathrm{G}_{\mathrm{m}}}{\mathrm{E}_{\mathrm{f}} \mathrm{d}^{2} \ln (2 \mathrm{R} / \mathrm{d})}\right)^{1 / 2}
\end{aligned}
$$

$\mathrm{G}_{\mathrm{m}}$ : shear modulus of the matrix which can be estimated

$$
\mathrm{G}_{\mathrm{m}}=\frac{\mathrm{E}_{\mathrm{m}}}{2\left(1+\mathrm{v}_{\mathrm{m}}\right)}
$$

$v_{\mathrm{m}}$ : The Poisson's ratio which equals to 0.35 for glass reinforced polycarbonate

$\mathrm{d}$ : The fibers diameter $=12 \mu \mathrm{m}$

2R: Center to center spacing of the fibers which for hexagonal packing arrangement

$$
\frac{\mathrm{d}}{2 \mathrm{R}}=\left(\frac{2(3)^{1 / 2} \phi_{\mathrm{f}}}{\pi}\right)^{1 / 2}
$$

From the experimental data we see that the influence of glass fiber concentration on the tensile modulus follows a straight line. The equation may also be reproduced as (5-7)

$$
E_{c}=E_{m}+\phi_{f}\left[E_{f} \eta_{L} \eta_{o}-E_{m}\right]
$$

The slope of the line equals $\left[E_{f} \eta_{L} \eta_{o}-E_{m}\right]$, and from the slope one can calculate $\eta_{o}$

Table 5.5 summarizes the orientation and length efficiency for virgin, recycled, and blend of the virgin and recycled glass reinforce polycarbonate. 
Table 5-5 Reinforcement efficiencies for glass reinforced polycarbonate

\begin{tabular}{|c|c|c|c|c|}
\hline \multicolumn{5}{|c|}{ Virgin Polycarbonate } \\
\hline$\% w t$ & $\% \phi_{\mathrm{f}}$ & $\mathrm{L}_{\mathrm{f}, \mu \mathrm{m}}$ & $\eta_{\mathrm{L}}$ & $\eta_{\mathrm{o}}$ \\
\hline 5 & 2.5 & 174 & 0.42 & 0.80 \\
\hline 10 & 5.1 & 172 & 0.46 & 0.72 \\
\hline 15 & 7.8 & 161 & 0.47 & 0.71 \\
\hline 20 & 10.7 & 156 & 0.48 & 0.69 \\
\hline \multicolumn{5}{|c|}{ Recycled Polycarbonate } \\
\hline wt & $\phi_{\mathrm{f}}$ & $\mathrm{L}_{\mathrm{f}, \mu \mathrm{m}}$ & $\eta_{L}$ & $\eta_{\mathrm{o}}$ \\
\hline 5 & 2.5 & 176 & 0.45 & 0.87 \\
\hline 10 & 5.1 & 171 & 0.48 & 0.81 \\
\hline 15 & 7.8 & 163 & 0.50 & 0.79 \\
\hline 20 & 10.7 & 155 & 0.50 & 0.78 \\
\hline \multicolumn{5}{|c|}{$50 \%$ Virgin $+50 \%$ Recycled Polycarbonate } \\
\hline wt & $\phi_{\mathrm{f}}$ & $\mathrm{L}_{\mathrm{f}, \mu \mathrm{m}}$ & $\eta_{\mathrm{L}}$ & $\eta_{\mathrm{o}}$ \\
\hline 5 & 2.5 & 173 & 0.42 & 0.91 \\
\hline 10 & 5.1 & 172 & 0.47 & 0.82 \\
\hline 15 & 7.8 & 159 & 0.47 & 0.82 \\
\hline 20 & 10.7 & 155 & 0.49 & 0.79 \\
\hline
\end{tabular}

In principle, fibers tend to align themselves in the direction of flow during injection molding. The orientation efficiency, $\eta_{\mathrm{o}}$, of the reinforcing fibers in Table 5-5 is consisting with fiber alignment during injection molding. Figure 5-14 shows the 
influence of impurities on the elastic modulus of polycarbonate and indicates that the effect is insignificant. It is also evident that, in a blend of polycarbonate and glass fiber that contains no more than $25 \%$ of impurities, the modulus of elasticity is not significantly affected. To predict the modulus of elasticity of glass-reinforced polycarbonate with impurities, the modulus of elasticity of the ureinforced polycarbonate with impurities and the orientation efficiencies of $15 \%$ glass reinforced virgin polycarbonate were used with rules of mixtures (equation 5-7) results are shown in Figure 5-14. Based on Figure 5-14, it appears that the modified rule of mixture can be used to predict the modulus of elasticity of glass reinforced polycarbonate with impurities. 


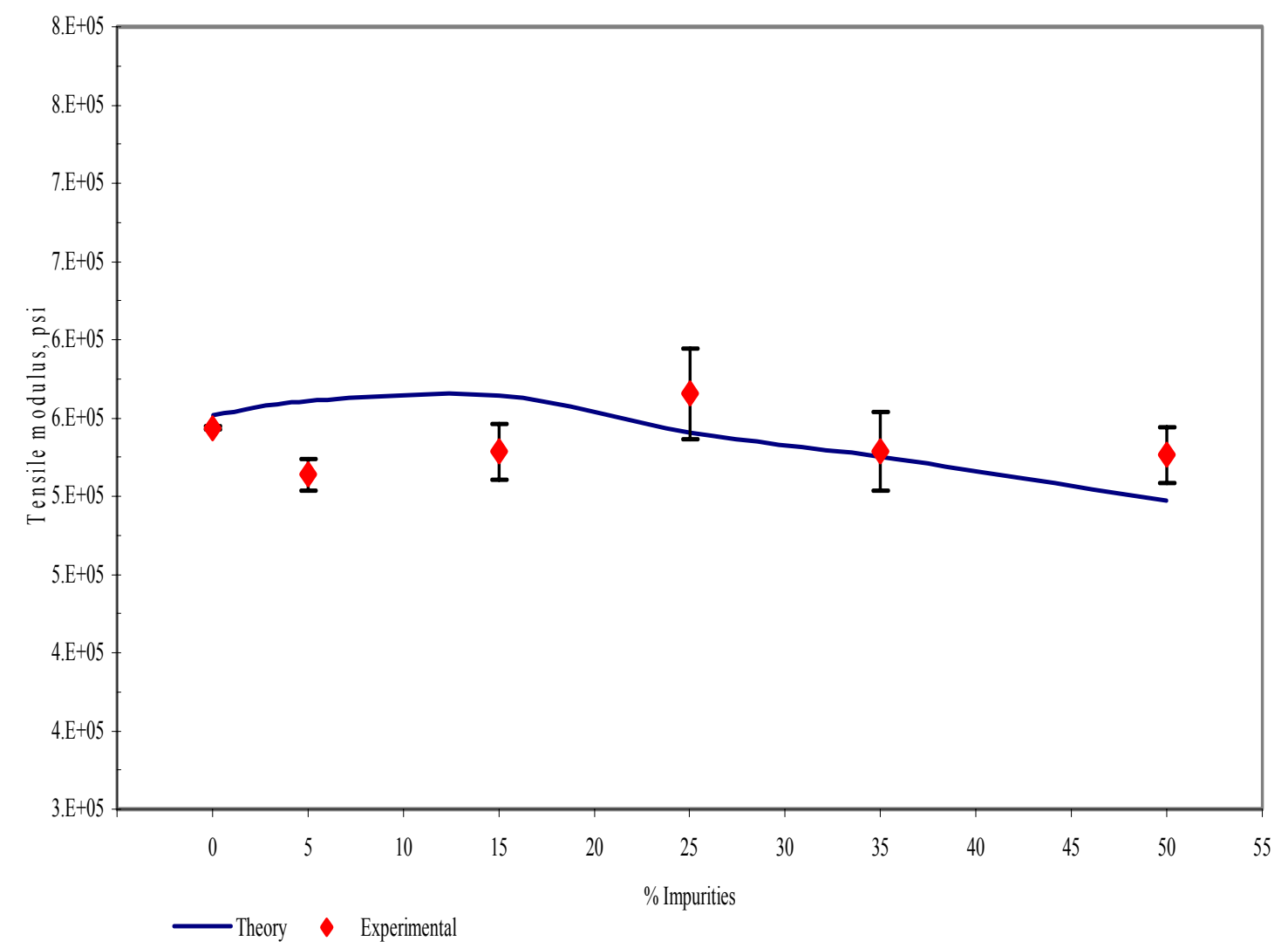

Figure 5-14 Effect of Impurities on the Modulus of Elasticity of 15\% glass reinforced polycarbonate

\subsubsection{Elongation at yield and break}

When glass fiber is added to polycarbonate, both the elongation at yield and break decrease as the content of glass fiber increases (see Figures 5-15 and 5-16). This is due to the poor elongation of glass fiber compared to the elongation of pure polycarbonate. As expected, virgin polycarbonate has a higher elongation than the recycled polycarbonate. Virgin polycarbonate with no glass fiber breaks at a strain equal to $120 \%$ (according to GE plastics), whereas recycled polycarbonate with no glass fiber breaks at strain equal to 
$6.52 \%$.

On measuring the fiber size and orientation, it was found that the addition of glass fiber to the recycled material reduces the disparity between the virgin and recycled material. Elongation at break for the unreinforced virgin polycarbonate is over $100 \%$, whereas for the unreinforced recycled material it is less than $10 \%$. This degree of difference will not be acceptable in the manufacturing of recycled polycarbonate parts that need to withstand a large elongation. The addition of glass fiber reduces this difference to approximately $2 \%$ in elongation at break. The addition of glass fiber to the recycled polycarbonate increases the fracture stress and modulus of elasticity at the same rate as in the virgin polycarbonate. So $5,10,15$, and $20 \%$ glass reinforced recycled polycarbonate are of the same strength, elasticity and stiffness as $5,10,15$, and $20 \%$ glass reinforced virgin polycarbonate. 


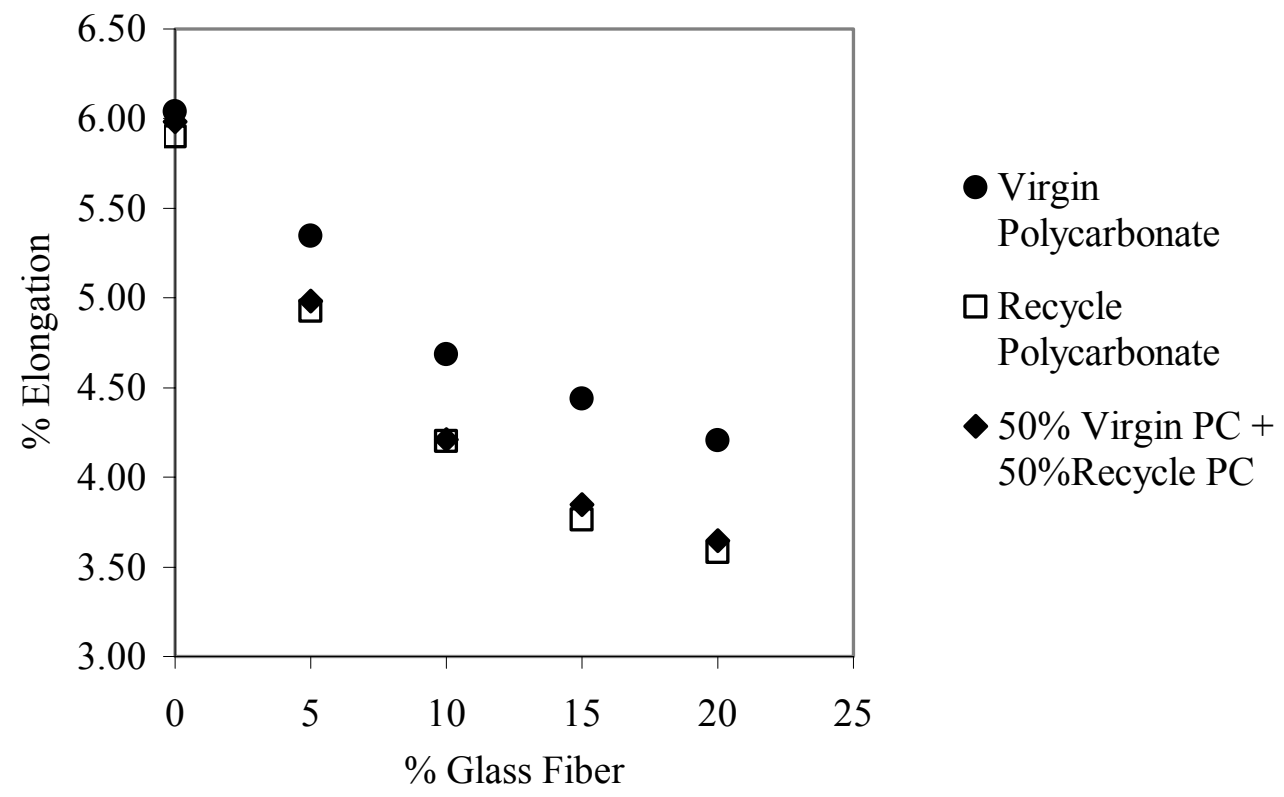

Figure 5-15 Effect of glass fiber on \% elongation at yield of polycarbonate

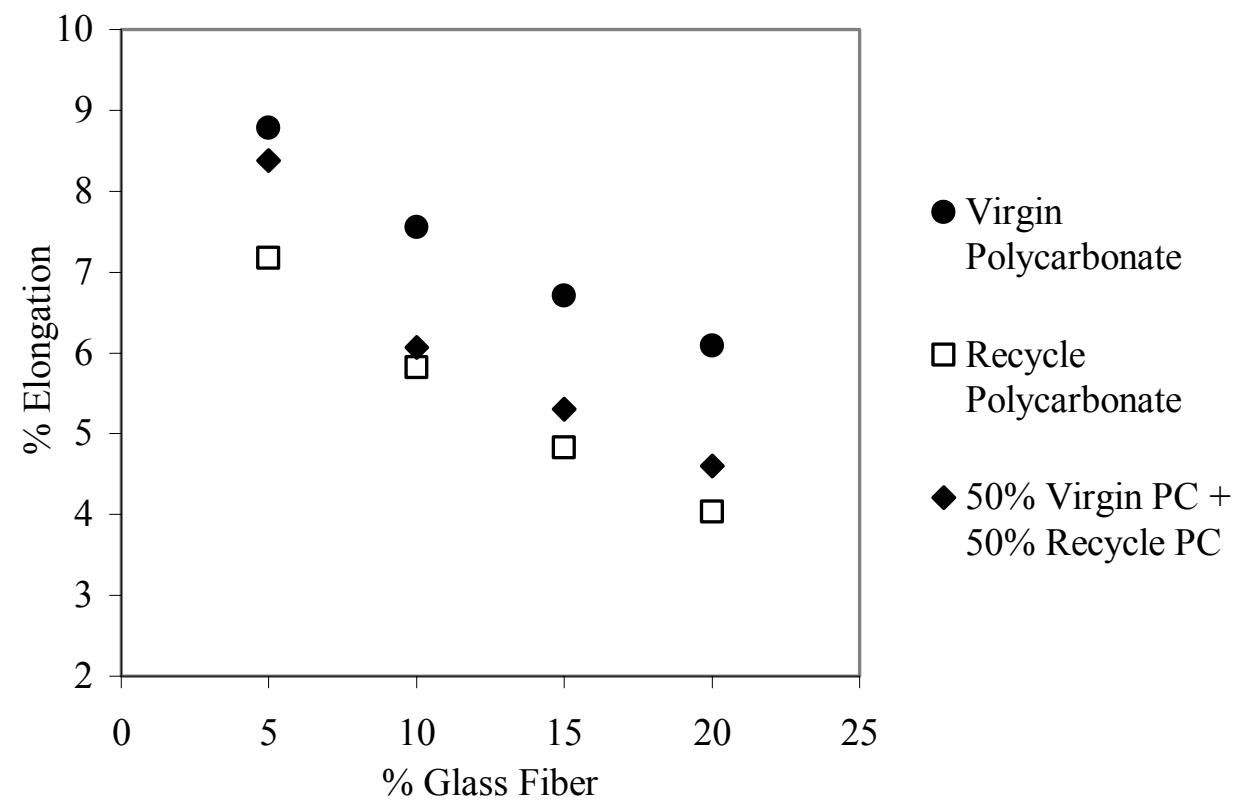

Figure 5-16 Effect of glass fiber on \% elongation at break polycarbonate 
The effect of impurities on the elongation at break of glass-reinforced polycarbonate is insignificant when the impurity content is below $15 \%$. As the amount of impurities exceeds $15 \%$ the elongation at break will reduce much more rapidly, as seen in Figure 5-17. Note that the elongation at break is a less-important property than the strength and modulus of elasticity for glass reinforced thermoplastics.

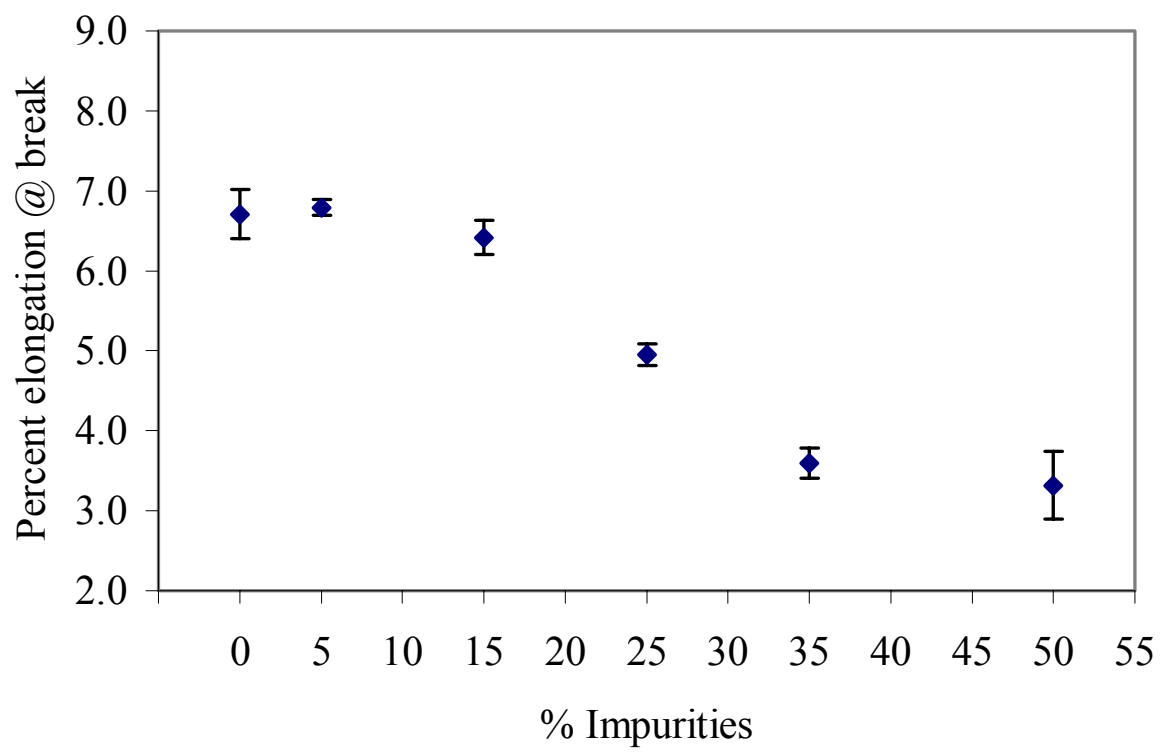

Figure 5-17 Influence of impurities on the ductility of $15 \%$ glass reinforced polycarbonate

\subsubsection{Flexural strength}

The orientation of glass fibers is usually in the direction of flow (see Figure 5-18). The tensile test measures the strength of material in tension and in the direction of glass fibers (see Figure 5-19). In the flexural test, force is applied perpendicular to the fiber 
direction (see Figure 5-20). The flexural strength is the unit resistance to the maximum load before failure by bending. Flexural strength was measured using the Instron Series 8500 machine with a head speed equal to $0.054 \mathrm{in} / \mathrm{min}$. The test was stopped when the deflection reached 5\% (according to ASTM).

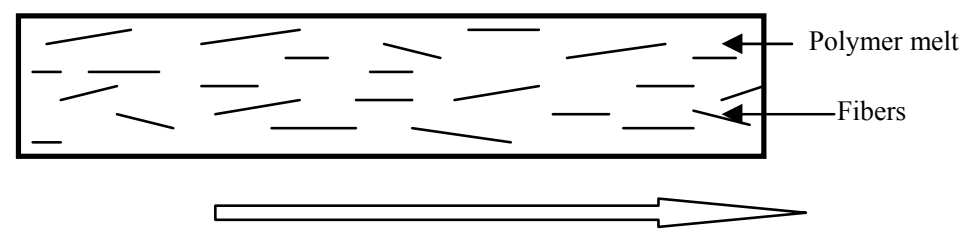

Direction of melt flow

Figure 5-18 Direction of fibers along the direction of flow

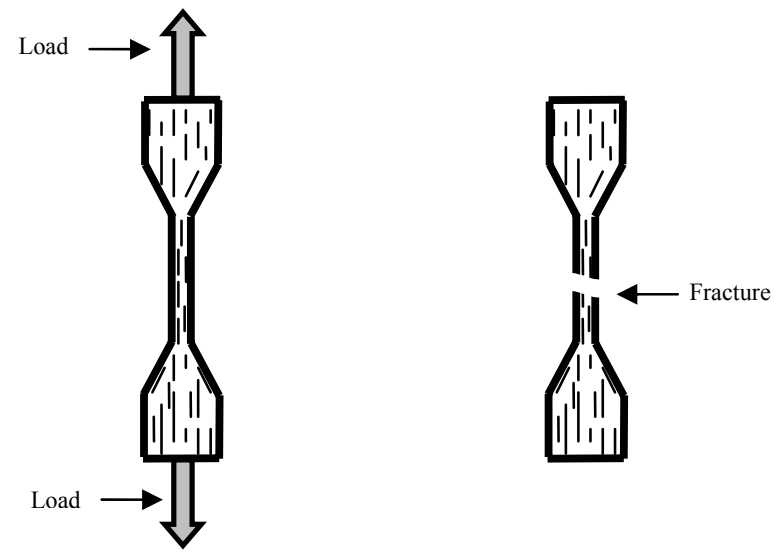

Figure 5-19 Direction of load in tensile test 

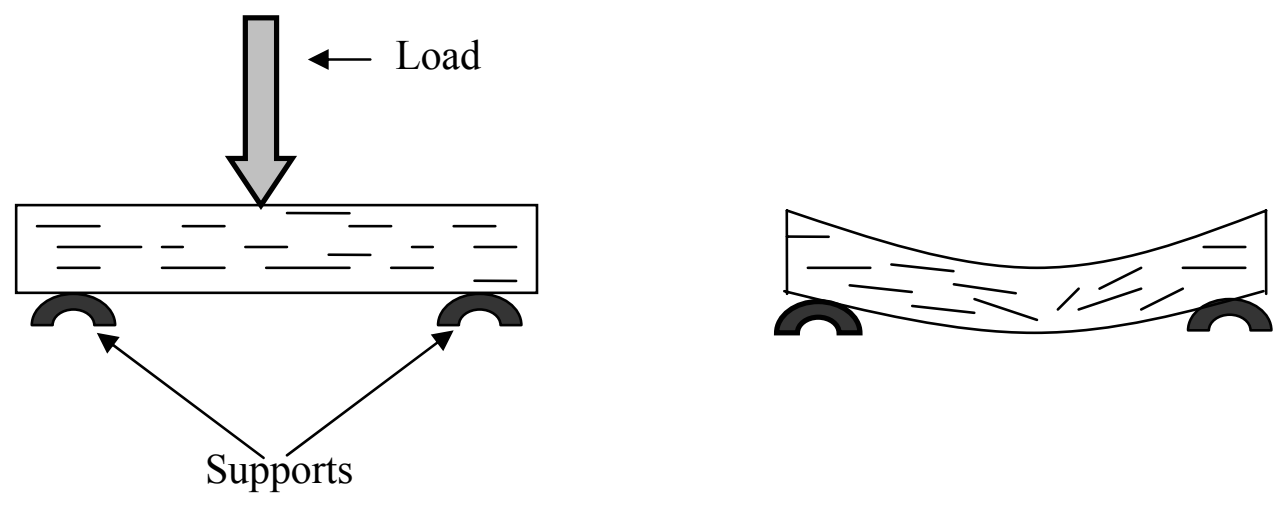

Figure 5-20 Direction of load in flexural test

When glass fiber is added to thermoplastics, the flexural strength increases in proportion to the amount of glass fiber added (see Figure 5-22). The addition of glass fiber has the same effect on recycled material as on virgin polycarbonate. The sample dimensions are:

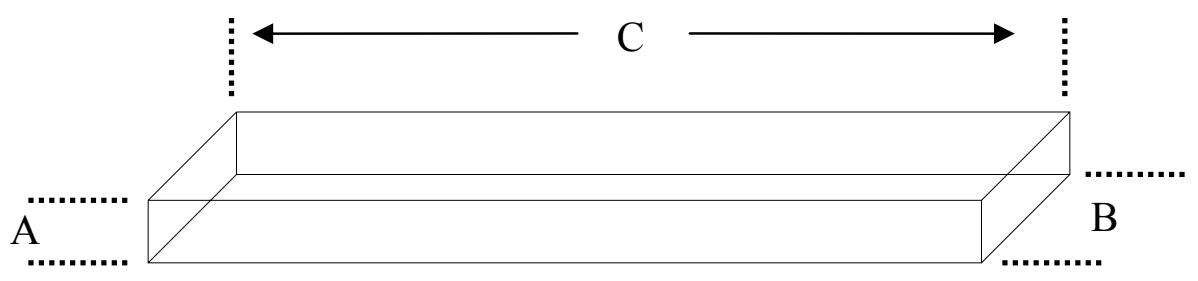

A: 0.125 inch, B: 0.5 inch, C: 5.0 inch

Figure 5-21 Flexural test sample dimensions 
The conclusion from Figure 5-22 is that the flexural strength of recycled polycarbonate is the same as virgin polycarbonate with $0 \%$ glass fiber. When the glass fiber is added to recycled polycarbonate the flexural strength increases at the same rate as for virgin polycarbonate. According to the observations based on analysis of plastic bending, rectangular cross-section beams can carry an additional $50 \%$ moment to that which is required to produce initial yielding at the edges of the beam section before a fully plastic hinge is formed.

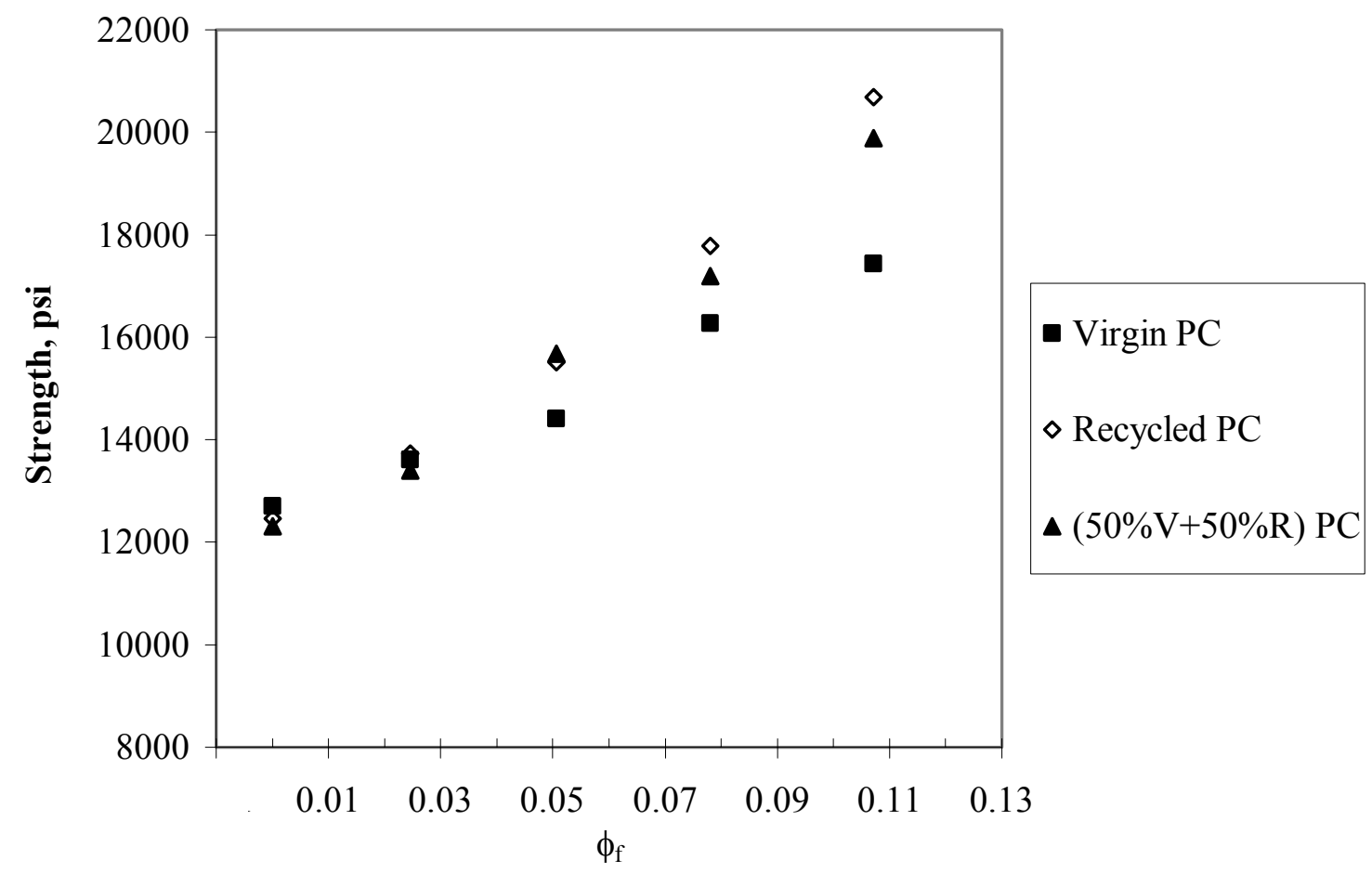

Figure 5-22 Effect of glass fiber on the flexural strength of polycarbonate

It is evident that the matrix has little effect on the flexural strength. Once again, a blend of polycarbonate and glass fiber containing no more than $25 \%$ of impurities does not significantly change the tensile strength as seen in Figure 5-23. 


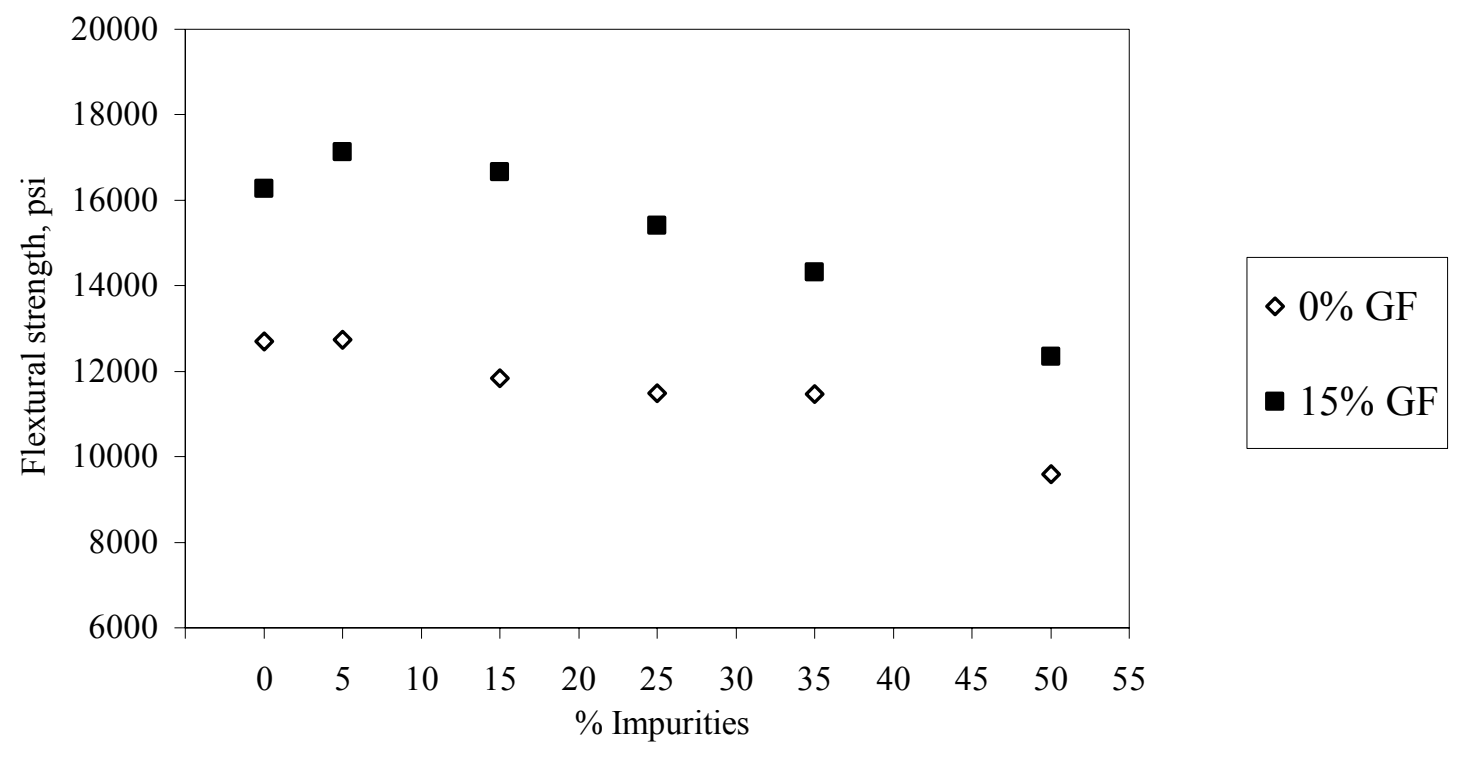

Figure 5-23 Influence of impurities on the flexural strength of un-reinforced and 15\% glass reinforced polycarbonate

\subsubsection{Impact strength}

Polycarbonate has a much higher impact strength compared to other thermoplastics. The impact strength of recycled polycarbonate is about $2 \mathrm{ft}-\mathrm{lb} /$ in whereas virgin polycarbonate has an impact strength of between 12-18 ft-lb/in (depending on the molecular weight of the sample). Recycled polycarbonate does, however, lose its high impact strength due to degradation of the material.

The impact test was conducted using a Satec system machine. The addition of glass fiber decreases the impact strength of virgin polycarbonate dramatically (see Figure 5-25). Since recycled polycarbonate has a low impact strength, the addition of glass fiber has little effect on the impact strength value (see Figure 5-25). As shown in Figure 5-25 it 
is hard to distinguish the difference in impact strength when using different glass fiber compositions. To determine the effect of glass fiber on the impact strength with different glass fiber compositions, an impact test was conducted on un-notched samples (see Figure 5-26). The purpose of performing an un-notched impact test was to measure the energy needed to initiate the crack, and the energy needed to propagate the crack in the samples. With the addition of glass fiber to recycled polycarbonate the difference in impact strength between virgin polycarbonate and recycled polycarbonate decreases dramatically. According to ASTM 256 the sample dimensions are:

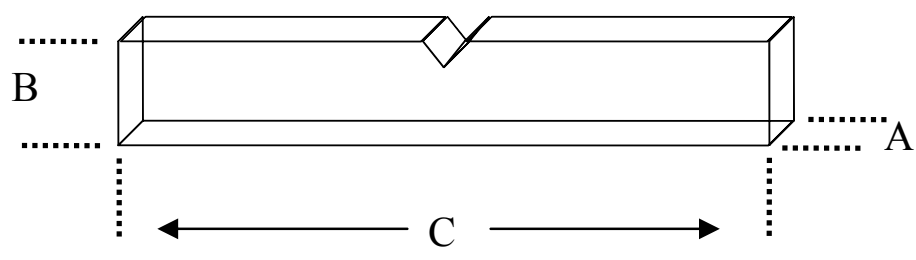

A: 0.125 inch, B: 0.5 inch, C: 2.5 inch

Figure 5-24 Impact test sample dimensions 


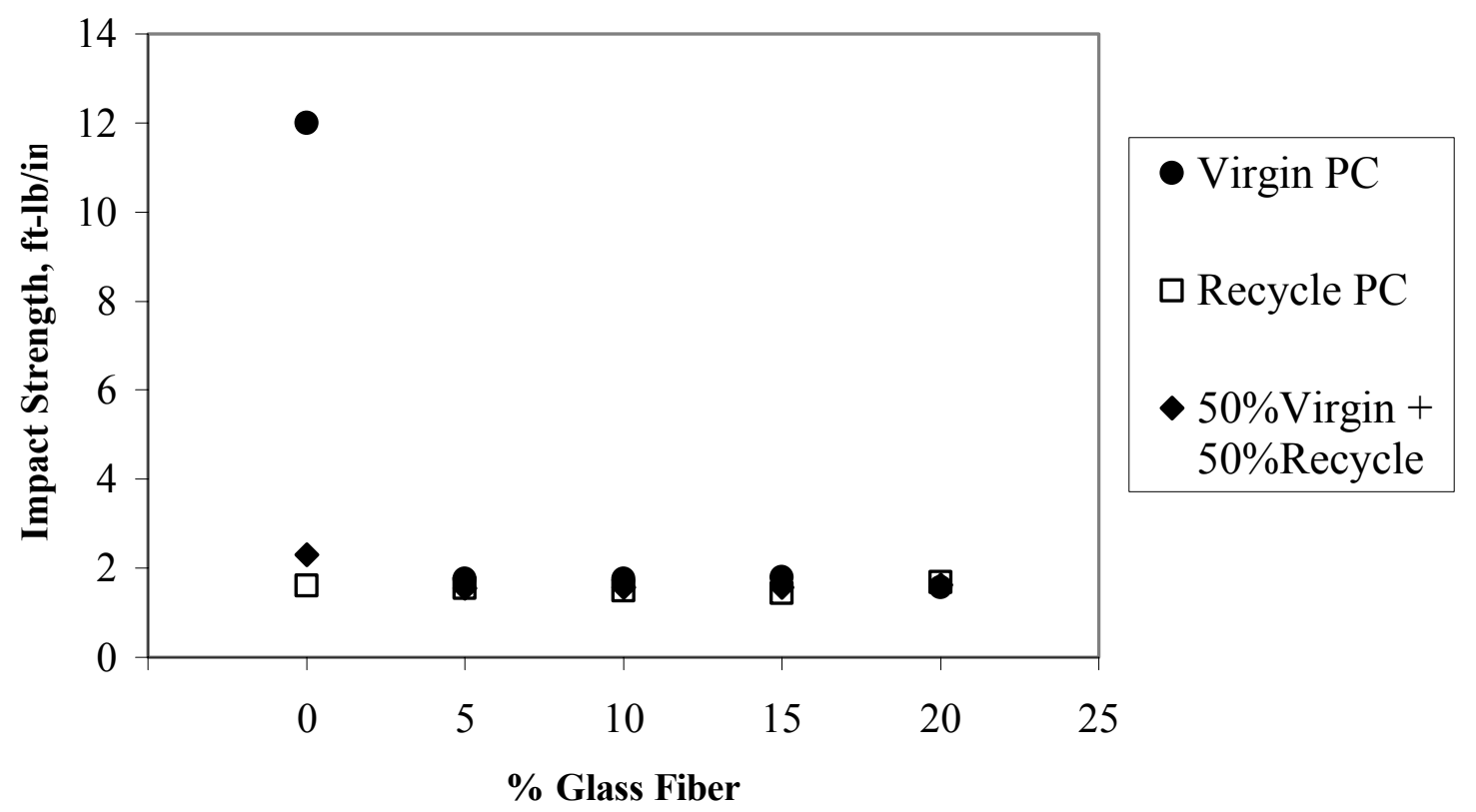

Figure 5-25 Effect of glass fiber on the impact strength of polycarbonate (samples are notched)

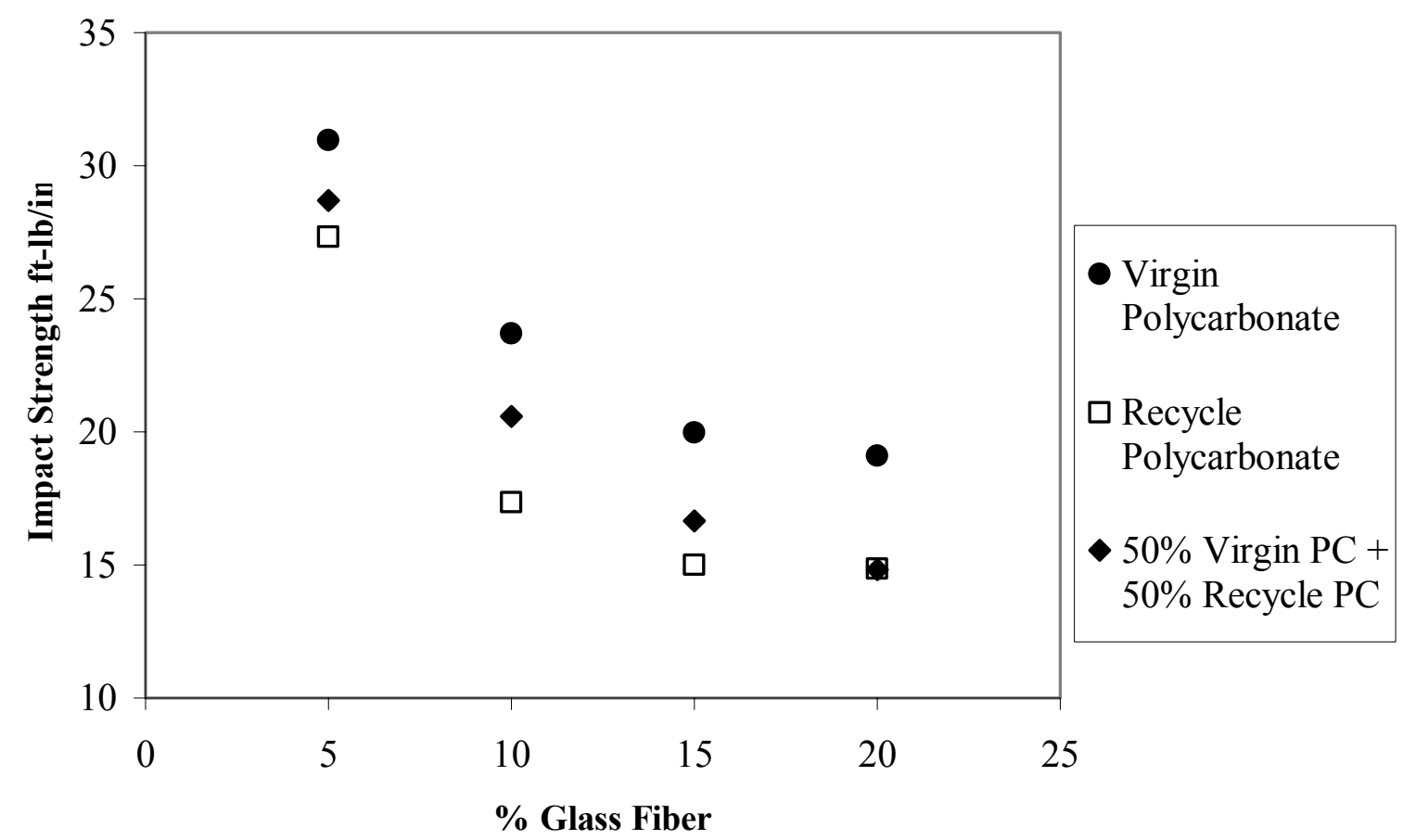

Figure 5-26 Effect of glass fiber content on impact strength of polycarbonate (samples are un-notched) 
When impurities are added to un-reinforced polycarbonate, there is a clear indication that the impact strength of the polymer matrix decreases drastically as seen in Figure 5-27. On the other hand, the influence of impurities on glass reinforced polycarbonate is very small. This indicates that the glass fiber is the dominant energy absorbent during the impact test.

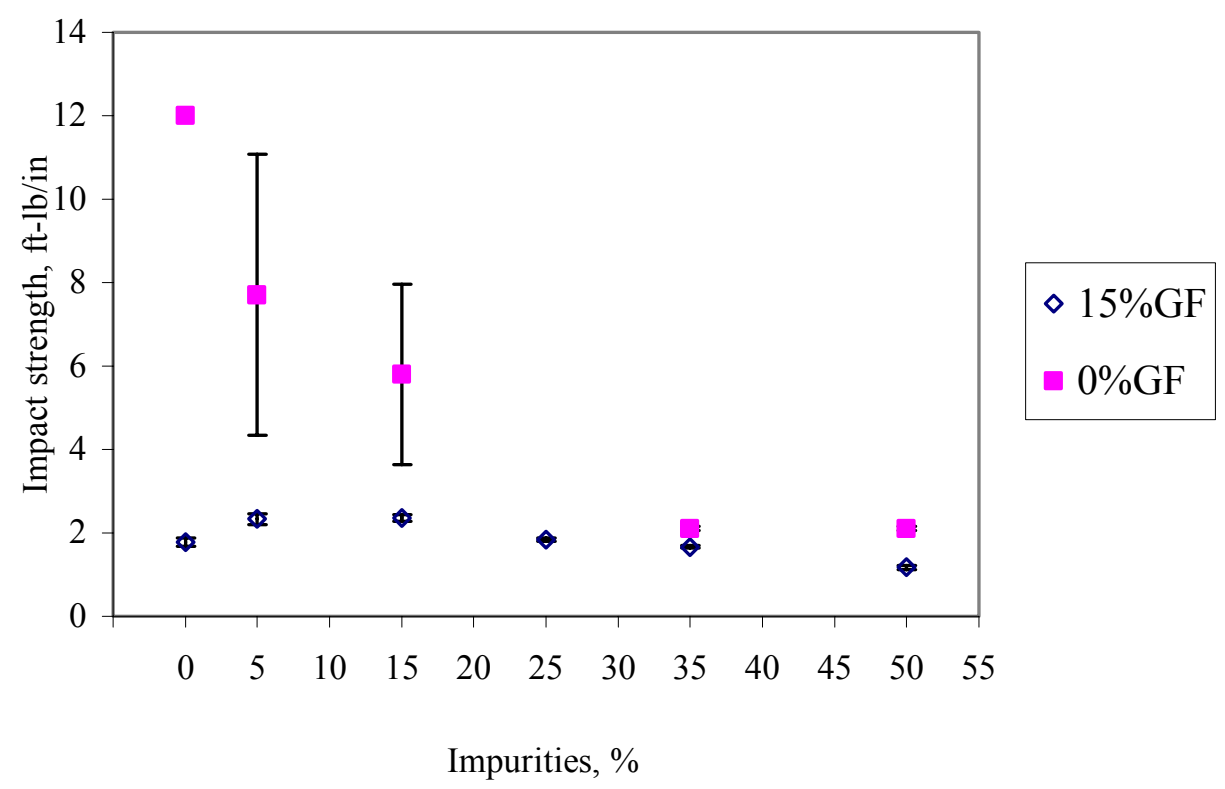

Figure 5-27 Influence of impurities on the notched impact test

\subsubsection{Hardness test}

The hardness testing of plastics is most commonly measured by the Shore (Durometer) test or Rockwell hardness test. Both methods measure the resistance of the plastic toward indentation. Both scales provide an empirical hardness value that does not 
correlate to other properties or fundamental characteristics.

The Shore hardness is measured with an apparatus called a Durometer (see Figure 5-28) and consequently is also known as "Durometer hardness". The hardness value is determined by the penetration of the Durometer indenter foot into the sample.

In Figure 5-29 one can see that the addition of glass fiber increases the hardness of the material. Recycled polycarbonate with no glass fiber content has a higher Shore hardness than virgin polycarbonate. This means that the recycled polycarbonate is harder than virgin polycarbonate. When glass fiber is added to the recycled polycarbonate however, the difference in hardness between virgin polycarbonate and recycled polycarbonate is reduced. This indicates that the addition of glass fiber makes the material less dependent on the type of the matrix phase than on the amount of glass fiber added.

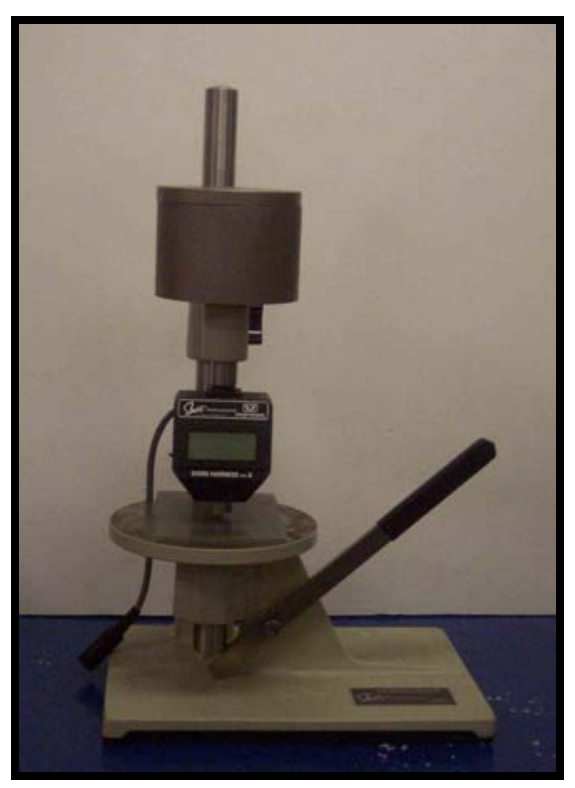

Figure 5-28 Durometer hardness used to measure the hardness of polycarbonate 


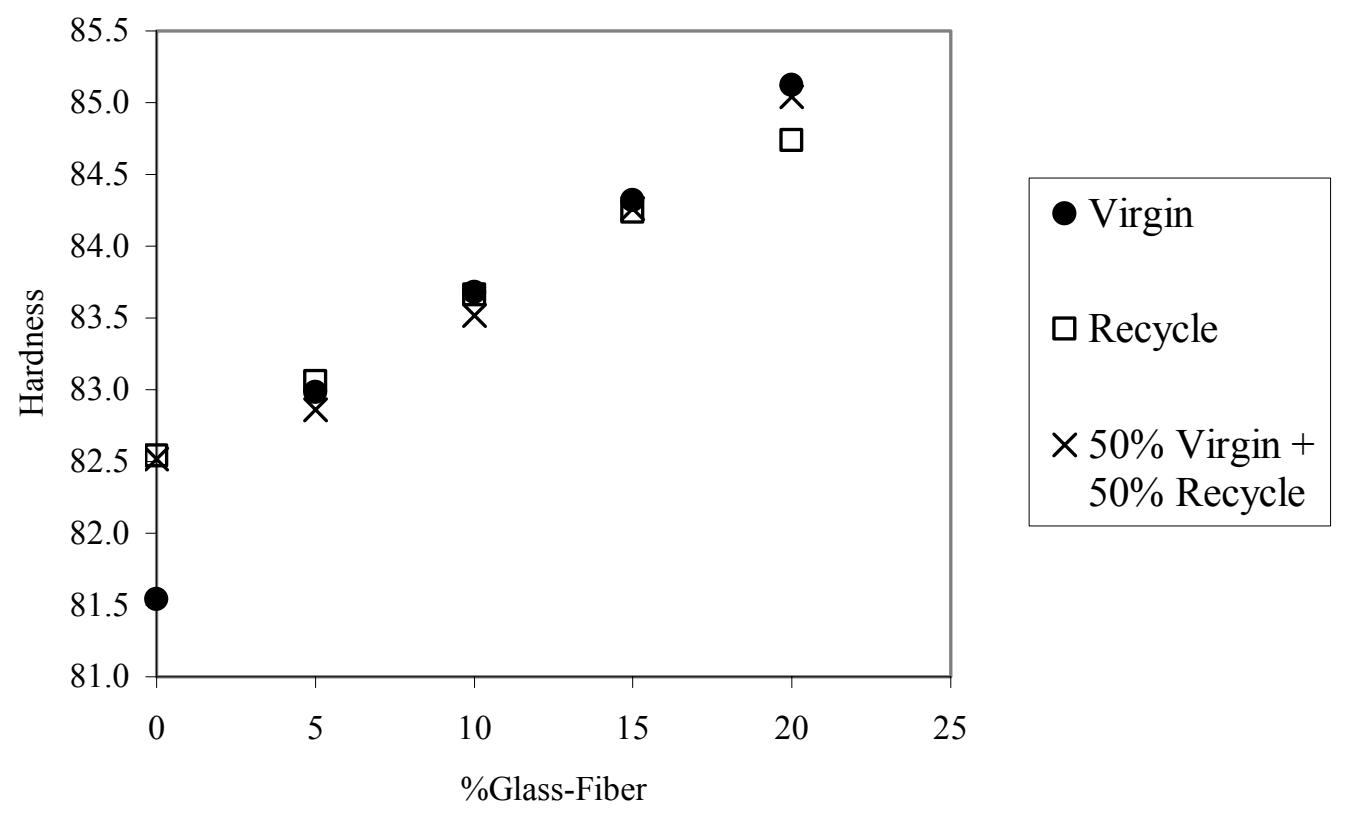

Figure 5-29 Effect of glass fiber on Shore's hardness

\subsection{Fracture surface analysis}

Fracture surfaces taken from tensile test specimens were analyzed by a Scanning Electron Microscope (SEM). SEM samples were coated with a thin film of gold alloy by sputtering. SEM was used to determine the effectiveness of the bond between the fiber and matrix. SEM allowe the degree of alignment of fibers in the samples to be seen. The fracture surfaces analysis gave a good indication about the bonding strength between polycarbonate and glass fiber, and whether this bonding is different for recycled polycarbonate and virgin polycarbonate. At least 15 samples for tensile tests were conducted using the SEM.

As seen in Figure 5-30 it is clearly evident from the fracture surfaces that fiber pull-out for all samples. The fiber pullout indicates weak interfacial bond between the 
fibers and the matrix (PC). Strong interfacial bond results in improved stiffness, higher strength but reduces ductility ${ }^{64}$. Figure 5-31 shows that in general fibers were aligned in the flow direction in the injection molding for tensile samples. Polycarbonate is ductile polymer and the appearance of the matrix fracture surface in the tensile test was ductile as seen in Figure 5-32. Recycled polycarbonate has less ductile fracture than the virgin polycarbonate (see Figure 5-33).

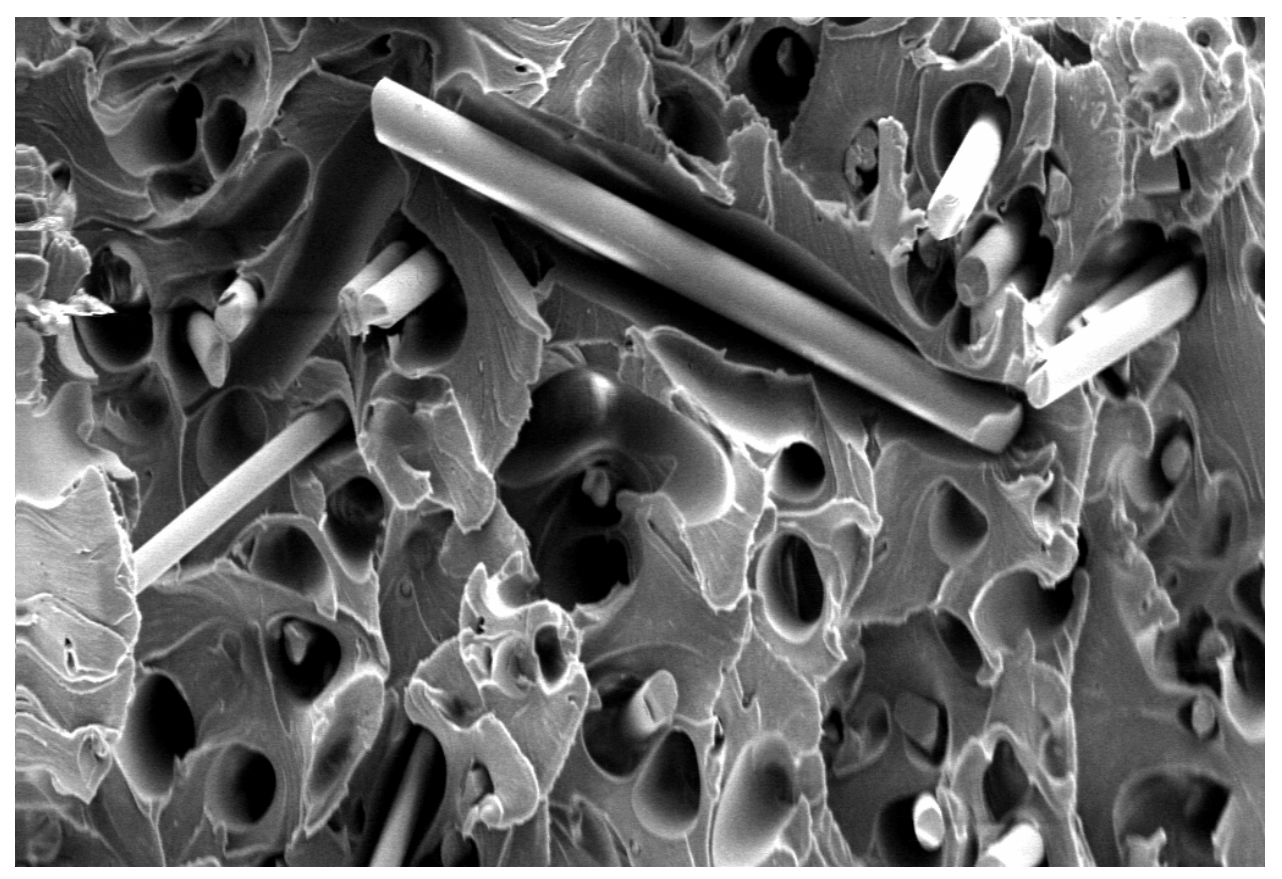

Figure 5-30 The fiber pull-out for 15\% glass fiber and mixture of virgin PC + recycled PC 


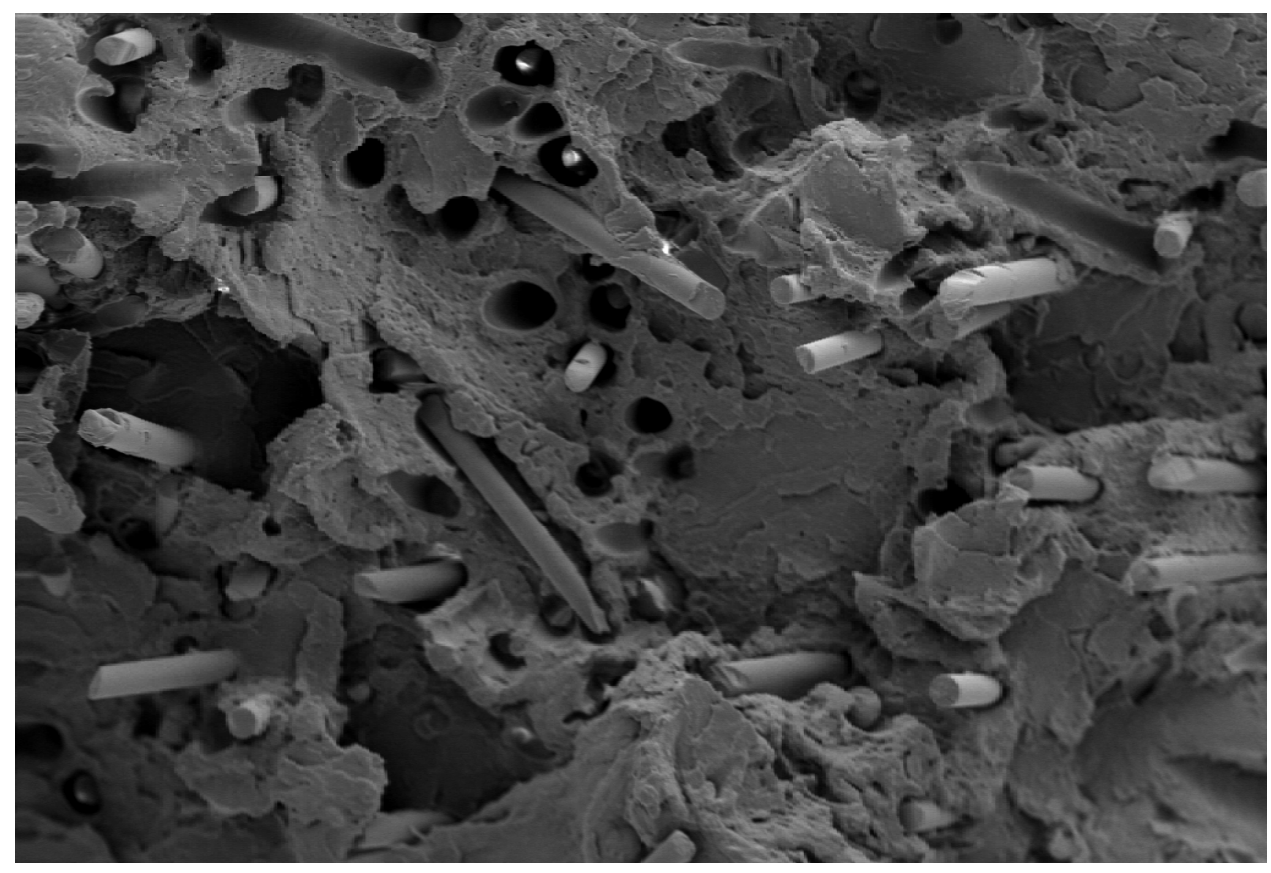

Figure 5-31 The alignment of glass fibers (15\% glass fiber and recycled PC) parallel to flow direction in injection molding

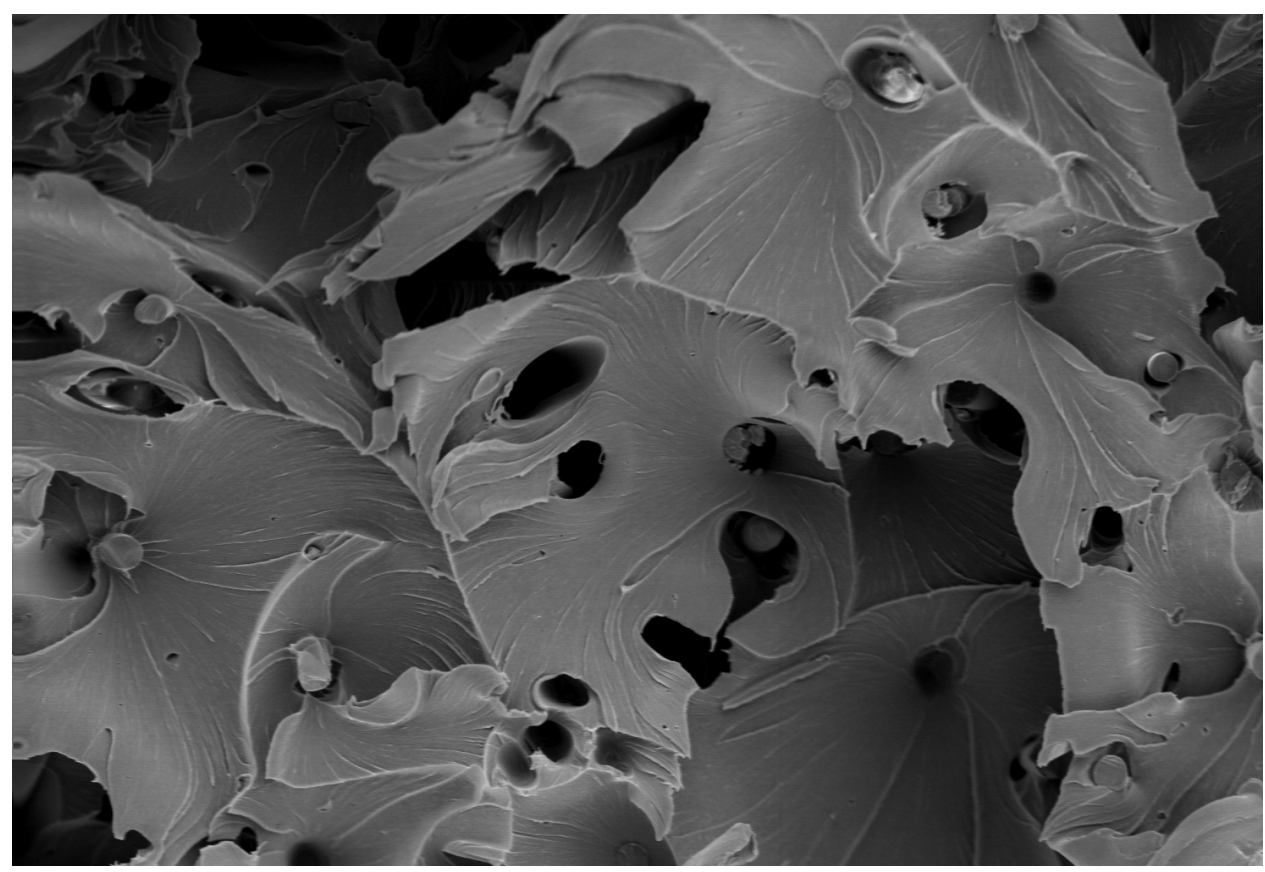

Figure 5-32 5\% glass fiber and virgin PC ductile fracture 


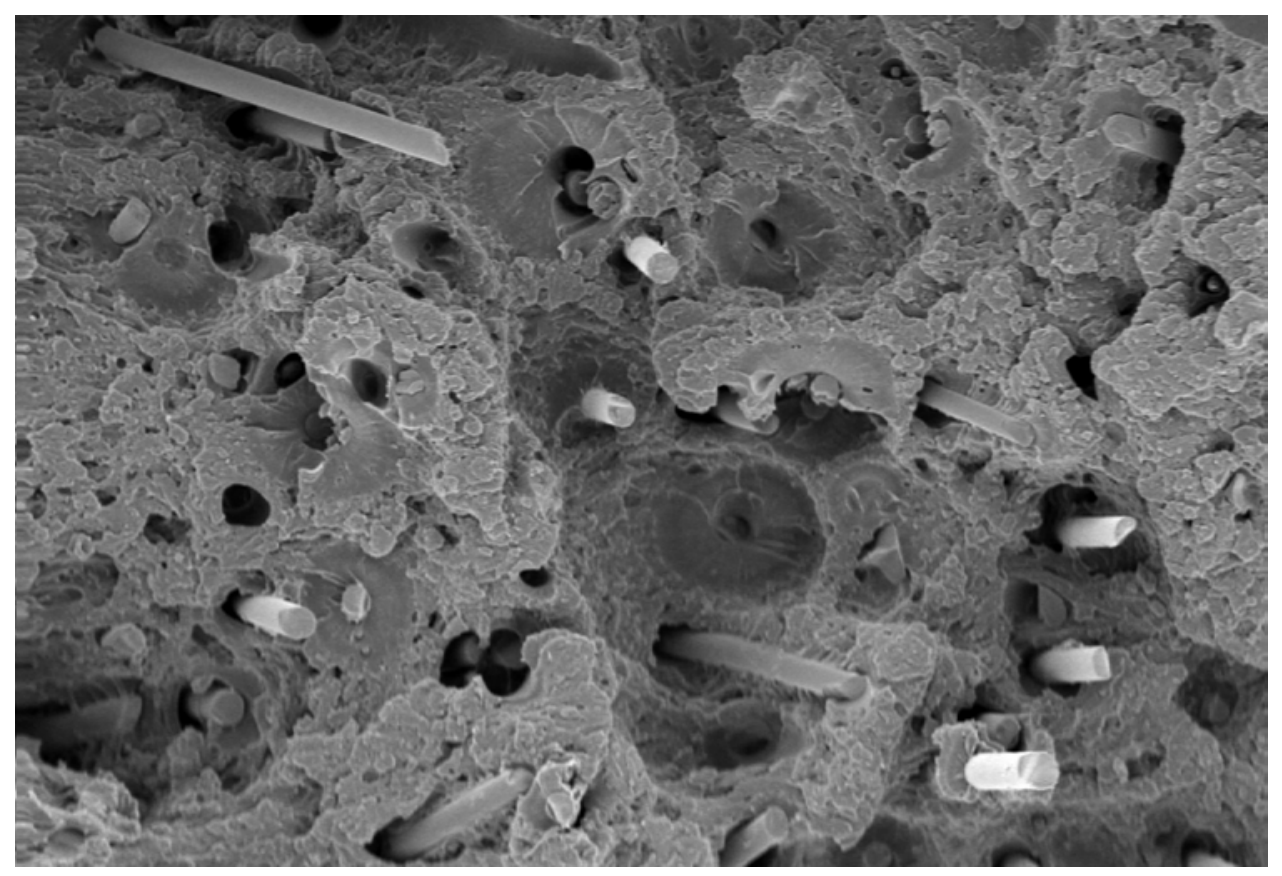

Figure 5-33 5\% glass fiber and recycled PC

Based on the results presented in this chapter, it can be concluded that the mechanical properties of recycled polycarbonate are improved by the addition of glass fiber. Preliminary results showed that when glass fibers were added to the recycled polycarbonate, the mechanical properties of glass reinforced recycled polycarbonate became close to those of glass reinforced virgin polycarbonate. This indicates that the mechanical properties depend more on the amount of glass fiber than on the type of matrix. These results were confirmed when impurities were added to polycarbonate. For un-reinforced polycarbonate the mechanical properties decreased with the addition of impurities. When the same impurities were added to glass reinforced polycarbonate, the mechanical properties changed very little. This suggests that in recycling thermoplastics there is no need to separate the different types of polymer but the blend of recycled thermoplastics can be combined with polycarbonate and glass fiber. 


\section{Rheological Results}

Several rheological tests were conducted on virgin, recycled, and blends of recycled and virgin polycarbonate. The aim of these tests was to study the rheological behavior of the three systems and to compare the behavior of the glass-reinforced virgin polycarbonate with glass-reinforced recycled polycarbonate. These tests were:

Low shear viscosity using a parallel plate viscometer

Dynamic mechanical tests

- Performing temperature sweep to measure the glass transition temperature, storage and loss modulus of the glass reinforced polycarbonate.

The shear viscosity was measured at a temperature of $260{ }^{\circ} \mathrm{C}$ and at shear rates between 0.1 and $1 \mathrm{~s}^{-1}$. This temperature was chosen because the processing temperatures for polycarbonate is between $250{ }^{\circ} \mathrm{C}$ and $300{ }^{\circ} \mathrm{C}$.

The dynamic mechanical tests were in two parts. The first part consisted of maintaining the samples at a temperature below the glass transition temperature (samples were solid). For the second part the samples were in a molten state (a temperature higher than $\mathrm{T}_{\mathrm{g}}$; this was $\sim 290{ }^{\circ} \mathrm{C}$ ). The loss and storage modului were measured for all 15 samples by conducting a temperature sweep between $40{ }^{\circ} \mathrm{C}$ and $160{ }^{\circ} \mathrm{C}$. From the storage modulus we obtained the glass transition temperature for all samples to discover whether $\mathrm{T}_{\mathrm{g}}$ for recycled $\mathrm{PC}$ is different from the $\mathrm{T}_{\mathrm{g}}$ for virgin PC. We also determined the influence of the glass addition to the $\mathrm{T}_{\mathrm{g}}$ of $\mathrm{PC}$.

One of the advantages of glass-reinforced thermoplastics over unreinforced thermoplastics is their dimensional stability. Glass-reinforced virgin polycarbonate has 
lower thermal expansion than unreinforced virgin polycarbonate at a given temperature. To ensure that this factor is the same in glass-reinforced recycled polycarbonate, a thermal expansion experiment was conducted. The thermal expansion was measured by conducting a temperature sweep between 40 and $120{ }^{\circ} \mathrm{C}$, and measuring the thermal expansion for all samples. The results were examined to compare the behavior of glassreinforced recycled polycarbonate with glass reinforced virgin polycarbonate. The second part, in which the samples were in the molten state, measured the loss and storage modului by conducting frequency sweeps at a temperature of 250,260 , and $290{ }^{\circ} \mathrm{C}$ for all samples. A comparison was made of the measured loss and storage modulus results between the virgin PC and the recycled PC; these results were then compared to other theoretical models.

\subsection{Melt flow rate}

The melt flow index (MFI) is defined as the weight of the polymer (in grams) extruded in 10 minutes through a capillary of a specific diameter and length, by pressure applied through dead weight under prescribed temperature conditions. For unreinforced polycarbonate the weight is $1.2 \mathrm{~kg}$ and the temperature is $300^{\circ} \mathrm{C}$. Table $6-1$ shows the result for virgin polycarbonate, recycled polycarbonate, and a blend of $50 \%$ virgin polycarbonate $+50 \%$ recycled polycarbonate. Recycled polycarbonate has a high MFI, indicating that the material is degraded or of low molecular weight. Table 6-2 shows the melt flow index for reinforced polycarbonate with a weight of $2.16 \mathrm{~kg}$ and a temperature of $300^{\circ} \mathrm{C}$. As the glass fiber content increases, the melt flow rate decreases. The glass- 
reinforced recycled polycarbonate has a higher melt flow index than the virgin polycarbonate. This difference is an indication that the recycled polycarbonate has been degraded and the molecular weight of recycled polycarbonate is lower than the molecular weight of virgin polycarbonate. The impurities tend to increase the melt flow index of the un-reinforced polycarbonate as shown in Figure 6-1. When impurities were added to glass reinforced virgin polycarbonate the melt flow index did not change as seen in Figure 6-1.

Table 6-1 Melt flow index of polycarbonate

\begin{tabular}{lc}
\hline \hline Polycarbonate & MFI ${ }^{*}$, gm/10min \\
\hline Virgin & 27.3 \\
Recycled & 57.3 \\
$50 \%$ Virgin $+50 \%$ Recycled & 37.2 \\
\hline \hline
\end{tabular}

$*$ Weight $=1.2 \mathrm{~kg}$, temperature $=300^{\circ} \mathrm{C}$

Table 6-2 Melt flow index of glass-reinforced polycarbonate

\begin{tabular}{cccc}
\hline \hline \% Glass fiber & \multicolumn{3}{c}{ Polycarbonate } \\
& Virgin & Recycled & $\mathbf{5 0 \%}$ Virgin + $\mathbf{5 0 \%}$ Recycled \\
\hline 5 & 47.13 & 94.97 & 64.85 \\
10 & 44.22 & 94.72 & 61.93 \\
15 & 48.72 & 64.2 & 58.8 \\
20 & 38.815 & 64.24 & 54.52 \\
\hline \hline
\end{tabular}




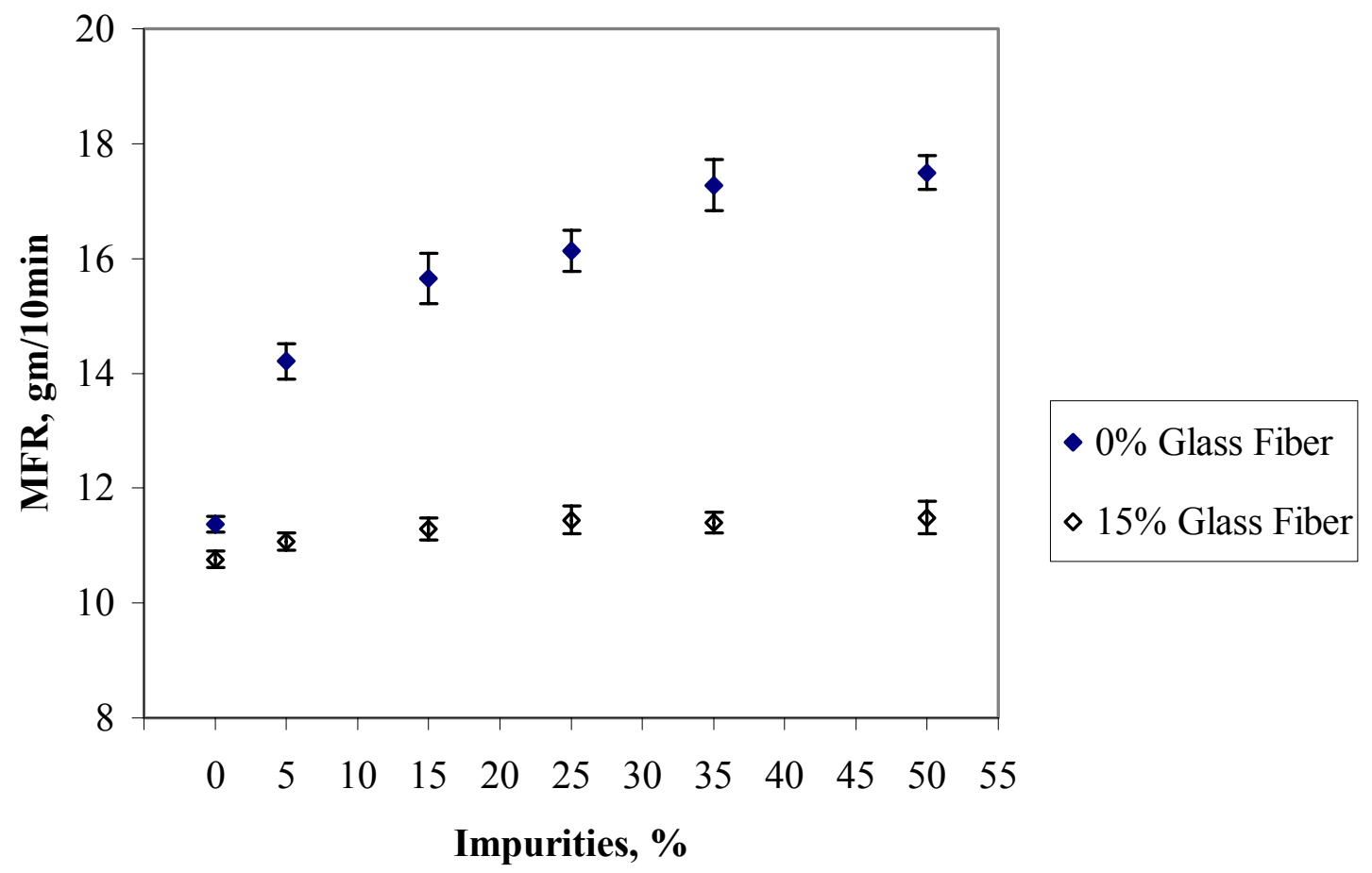

Figure 6-1 Influence of impurities on the melt flow index of virgin $\mathrm{PC}\left(\mathrm{T}=260{ }^{\circ} \mathrm{C}\right.$, load $=2.16 \mathrm{~kg}$ )

\subsection{Shear viscosity using melt flow indexer}

Shear viscosity is measured using a melt flow indexer at a temperature equal to $300^{\circ} \mathrm{C}$ and different weights. To measure the viscosity, the shear stress and the shear rate must be calculated using equations (6-1) and (6-2) ${ }^{65}$. The melt flow indexer can be used to measure the flow rate at an applied weight. The shear rate can be calculated using the flow rate and equation 6-2.

$$
\tau=\frac{\mathrm{R}_{\mathrm{N}} \mathrm{F}}{2 \pi \mathrm{R}_{\mathrm{P}}^{2} \mathrm{~L}_{\mathrm{N}}}
$$




$$
\dot{\gamma}=\frac{4 \mathrm{Q}}{\pi \mathrm{R}_{\mathrm{N}}^{3}}
$$

where

$\tau$ : Shear stress

$\mathrm{R}_{\mathrm{N}}$ : Nozzle radius $(0.105 \mathrm{~cm})$

$\mathrm{R}_{\mathrm{p}}$ : Piston radius $(0.4737 \mathrm{~cm})$

F: Test load

$\mathrm{L}_{\mathrm{N}}$ : Nozzle length $(0.8 \mathrm{~cm})$

Q: Flow rate $\left(\mathrm{cm}^{3} / \mathrm{s}\right)$

We see from Figure 6-2 that the addition of glass fiber increases the viscosity for virgin polycarbonate. Similar results are obtained for recycled polycarbonate and a blend of virgin and recycled polycarbonate. It can also be observed that recycled polycarbonate has a lower viscosity than virgin polycarbonate at the same shear rate and temperature (see Figure 6-3). When recycled polycarbonate is blended with virgin polycarbonate, the viscosity of the blend is higher than that of the recycled polycarbonate, but lower than the viscosity of the virgin polycarbonate (see Figure 6-4). This is a significant factor in the improvement of the rheological properties of recycled polycarbonate.

The viscosity measured so far is in a narrow shear rate range of $30 s^{-1}-130 s^{-1}$ which is due to the melt indexer machine limit, but we need to cover a wider shear rate. This can be accomplished using a parallel plate viscometer for low shear rates and a capillary viscometer for high shear rates. 


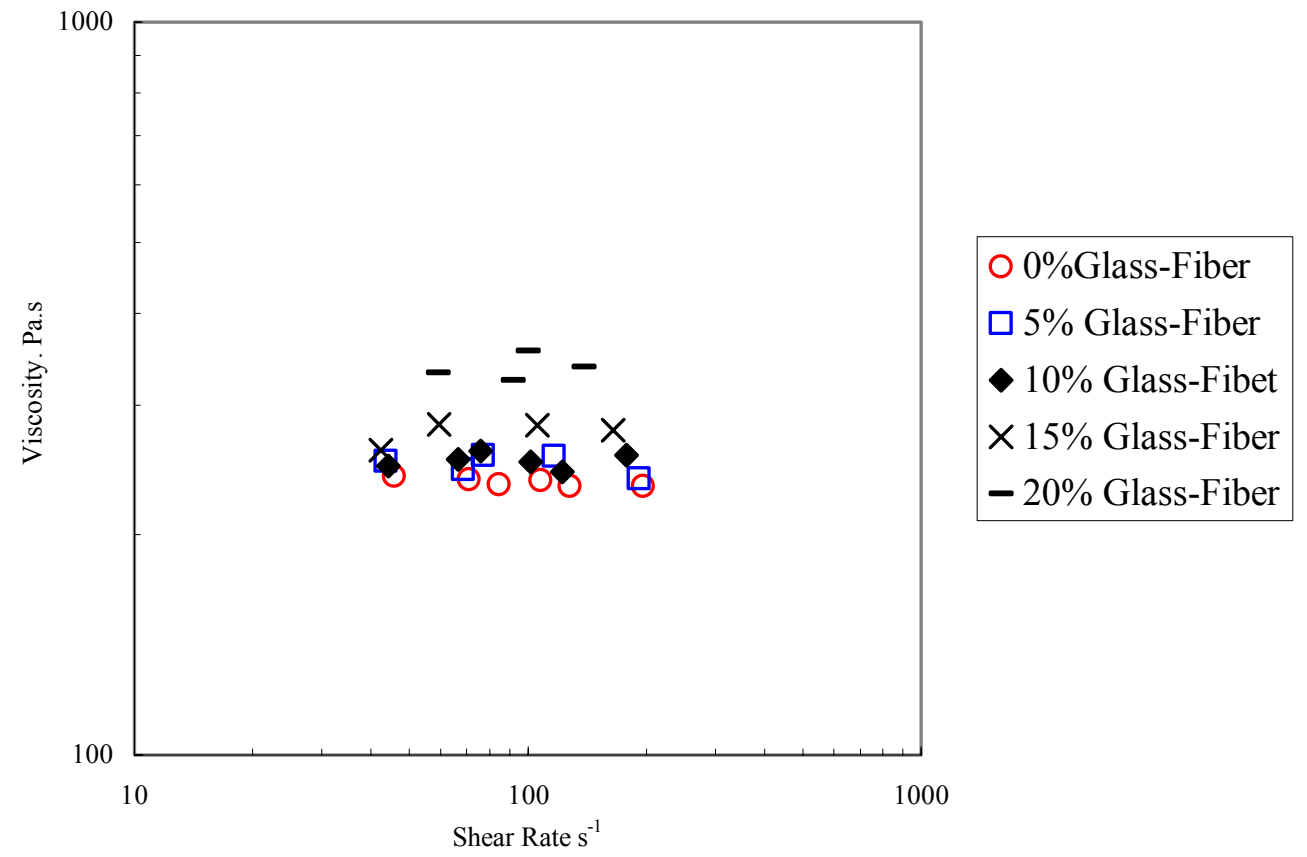

Figure 6-2 Effect of glass fiber on the shear viscosity of virgin polycarbonate

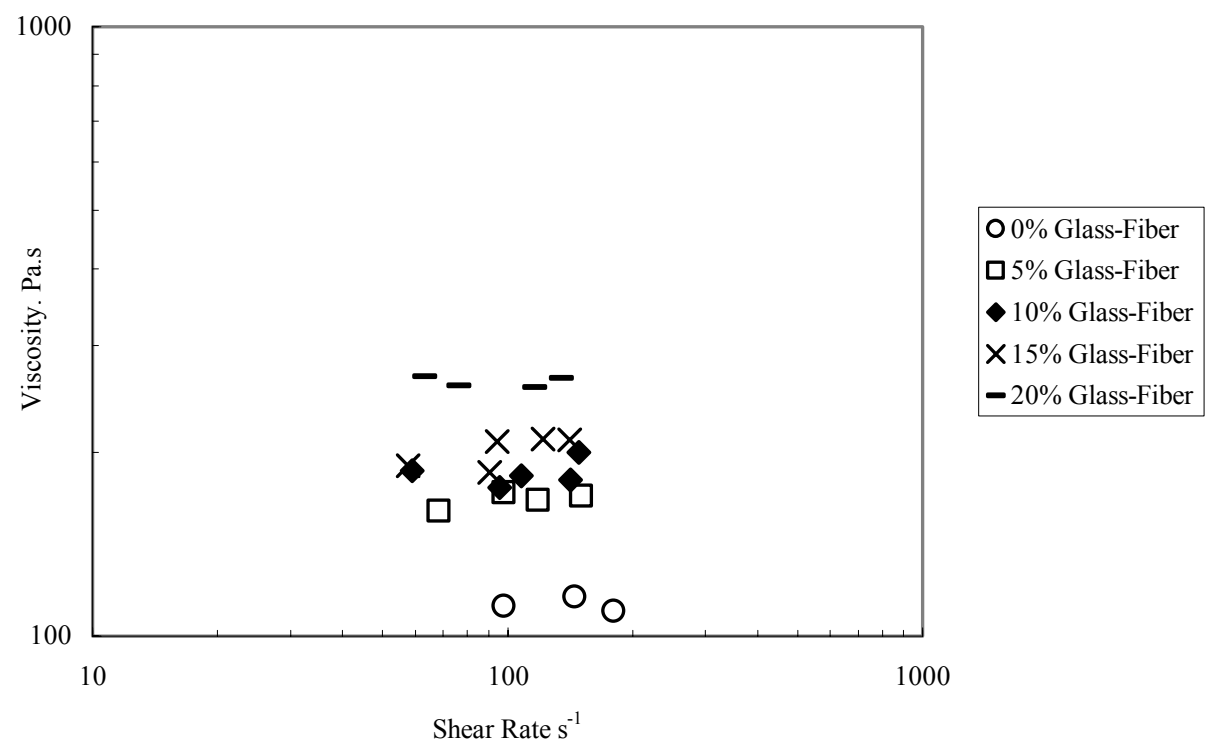

Figure 6-3 Effect of glass fiber on the shear viscosity of recycled polycarbonate 


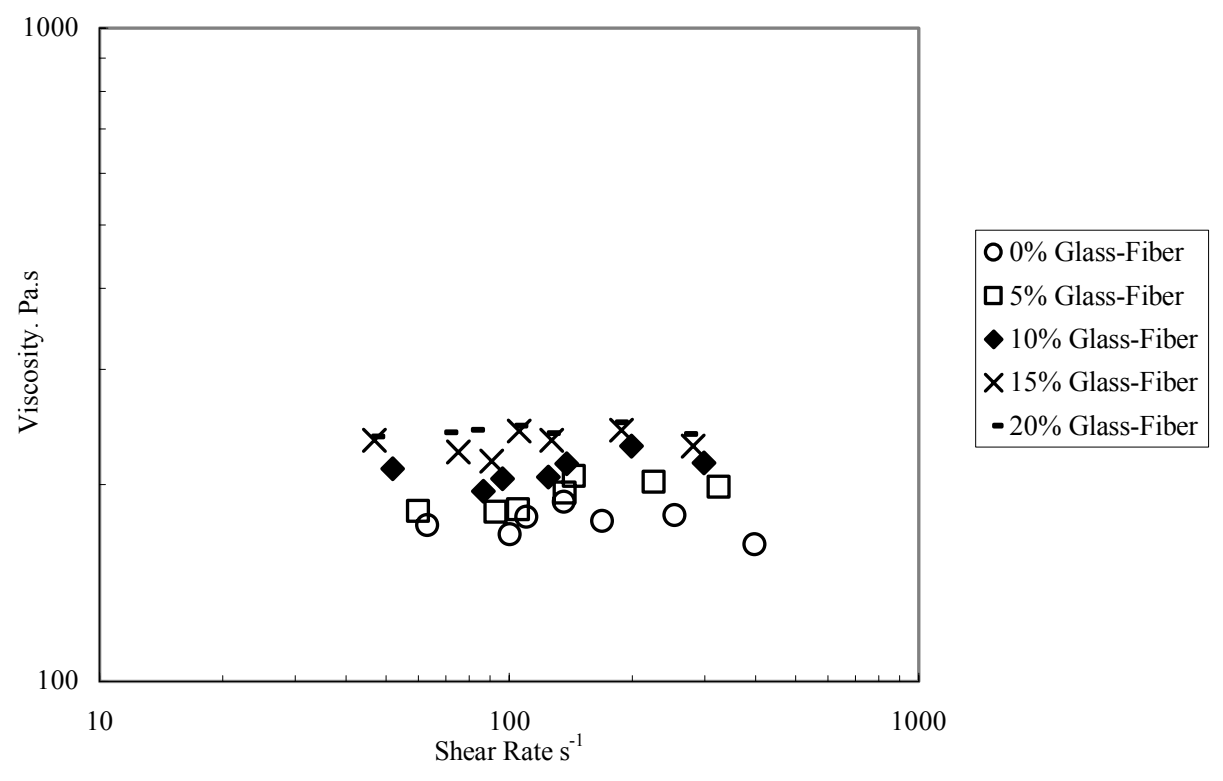

Figure 6-4 Effect of glass fiber on the shear viscosity of 50\% virgin polycarbonate $+50 \%$ recycled polycarbonate

\subsection{Steady shear viscosity}

The Rheometrics Mechanical Spectrometer (RMS-800) was used to measure transient and steady-state viscous properties of the polymers. The viscosity, shear stress, storage modulus, and loss modulus were measured using a parallel-plate viscometer. The upper disk was rotated at an angular velocity $(\omega)$ and the lower disk was fixed. The parallel-plate apparatus was used instead of the cone and plate apparatus, because the length of the fiber was greater than the gap in the cone and plate. The gap between the parallel plates must be greater than the length of the fiber (gap/length ratio $>1.0$ ), so that the fiber is not constrained by the walls ${ }^{\mathbf{6 6}}$. 


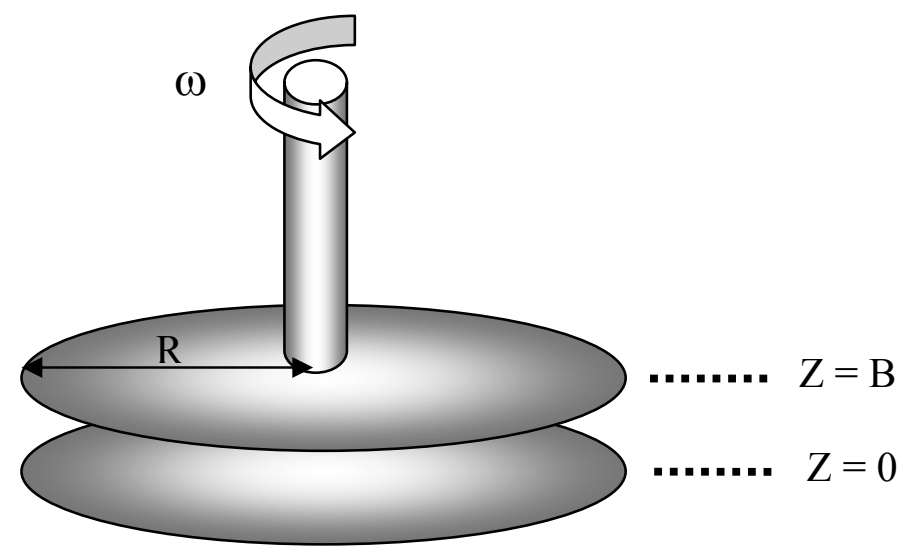

Figure 6-5 Parallel plate viscometer with two disks of radius $\mathrm{R}$ separated by a distance $\mathrm{B}$, with dimensions such that $\mathrm{R}>>\mathrm{B}$.

The parallel plate instrument is limited by the fact that the shear rate is not constant and varies linearly across the radius (see Figure 6-5). This is accounted for in the equations for the rheometric properties. The viscosity was obtained from measurements at various shear rates according to the following equations:

Viscosity:

$$
\eta(\dot{\gamma})=\frac{\mathrm{T}}{\dot{\gamma}_{\mathrm{R}}}\left[3+\frac{\mathrm{d} \ln \left(\frac{\mathrm{T}}{2 \pi \mathrm{R}^{3}}\right)}{\mathrm{d} \ln \dot{\gamma}_{\mathrm{R}}}\right]
$$

Shear rate:

$$
\gamma_{\mathrm{R}}=\frac{\omega_{0}}{\mathrm{~B}} \mathrm{R}
$$

Here $\mathrm{R}$ is the radius of the disks, $\mathrm{B}$ is the separation of the disks, $\omega$ is the angular velocity 
of the upper disk, and $\mathrm{T}$ is the torque required to rotate the upper disk. The radius is fixed at $12.5 \mathrm{~mm}$ and the gap set at $1.0 \mathrm{~mm}$.

The viscosity at steady state is a measure of the fluid's resistance to flow, and is a well-studied characteristic of many materials, including polymers. The non-Newtonian viscosity in shear flows is primarily a function of temperature and shear rate. At higher temperatures and higher fluid velocities, and hence shear rates, the polymer molecules are less entangled and thus flow more easily. At lower temperatures and speeds, the polymer molecules behave more as a solid and resist flow. The addition of fibers or other reinforcing particles generally hinders flow and causes an increase in viscosity. However, as the fibers orient with the flow, the resistance decreases. In this chapter, the effect of the fibers on the viscous properties of the polymers during steady testing is analyzed.

The viscosity levels for the materials are determined from equation (6-3). The procedure will be described later in this chapter. Care was taken to ensure that the average gap was similar for each material, since the shear rate (and hence the measured viscosity) vary across the radius. The viscosity levels for unreinforced and reinforced polycarbonate at various glass weight percentages are shown in Figures 6-6, 6-7, and 6-8. For all three materials, the short fibers significantly increase the viscosity at a low shear rate. Previous work supports the increase in viscosity at low shear rates. Note that flow instabilities arise and material leaves the gap at higher shear rates. This was observed at glass concentrations of $10 \%, 15 \%$, and, $20 \%$ for all three resins when the shear rate was greater than $20 \mathrm{sec}^{-1}$. Previous researchers used parallel plates at low shear rates and a capillary rheometer at higher shear rates. A capillary rheometer was not available for use in this research. 


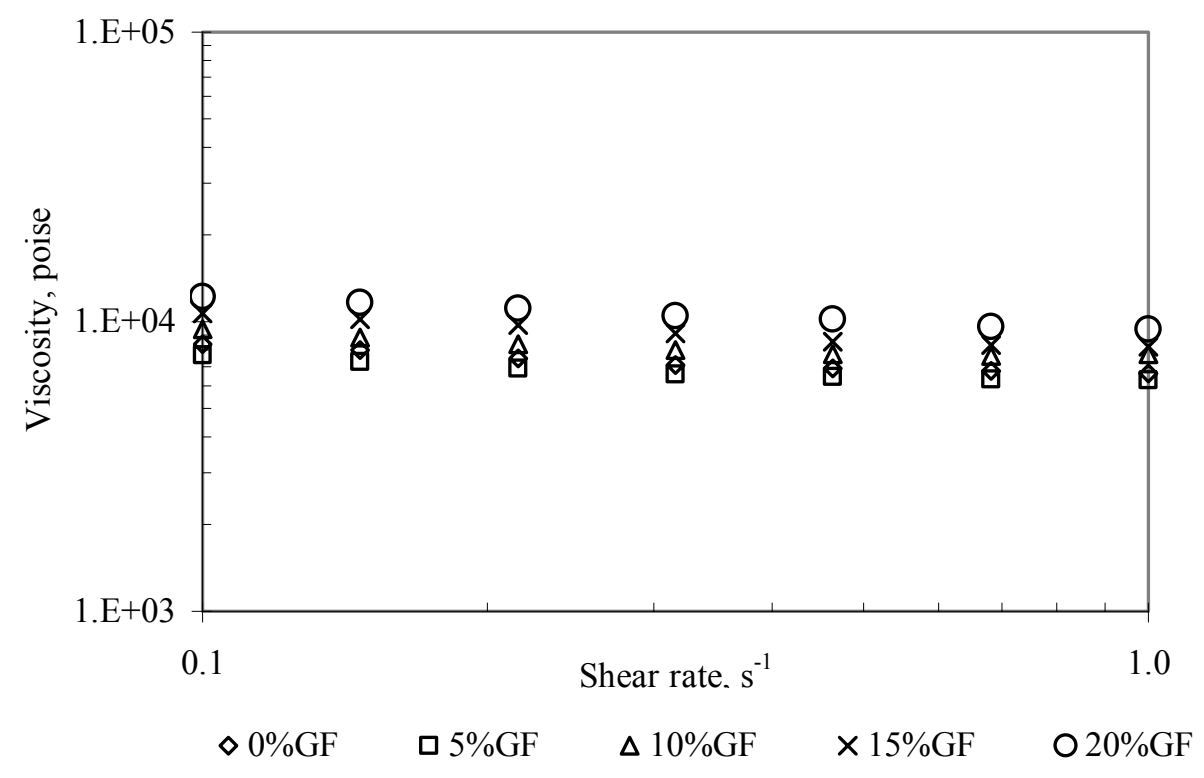

Figure 6-6 Virgin polycarbonate steady-state viscosity at temperature $260{ }^{\circ} \mathrm{C}$

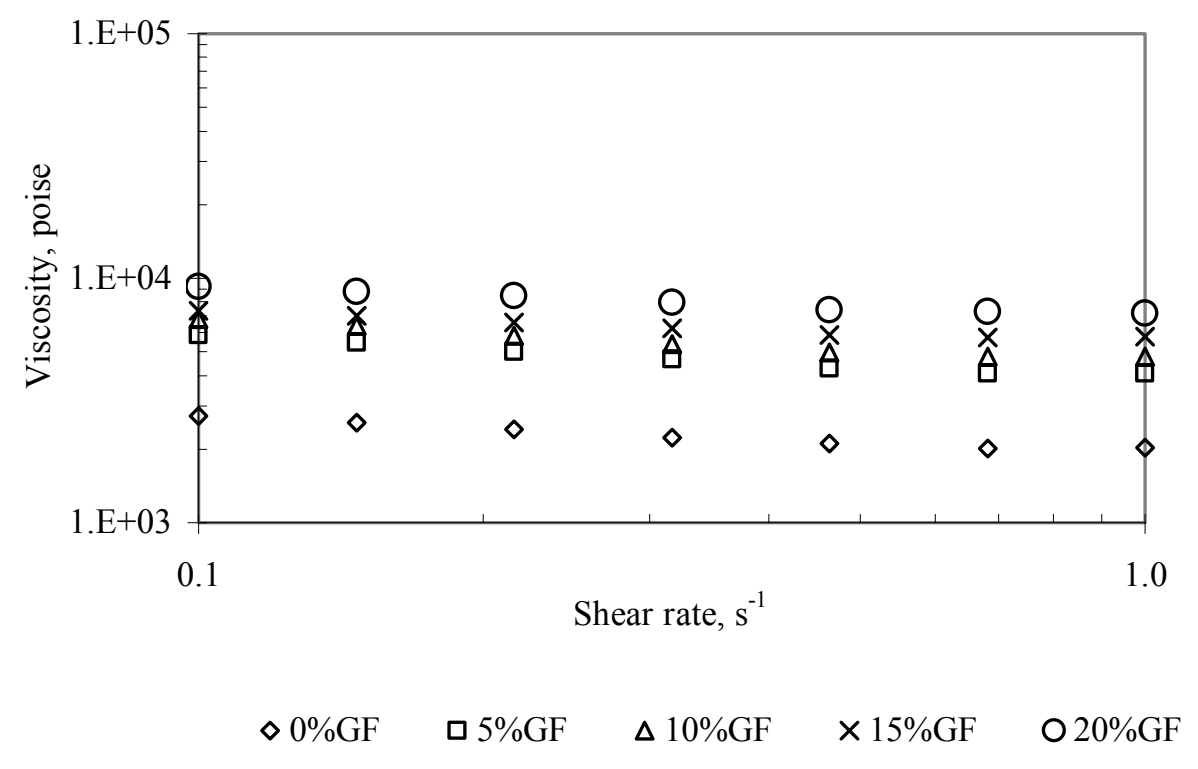

Figure 6-7 Recycled polycarbonate steady-state viscosity at temperature $260{ }^{\circ} \mathrm{C}$ 


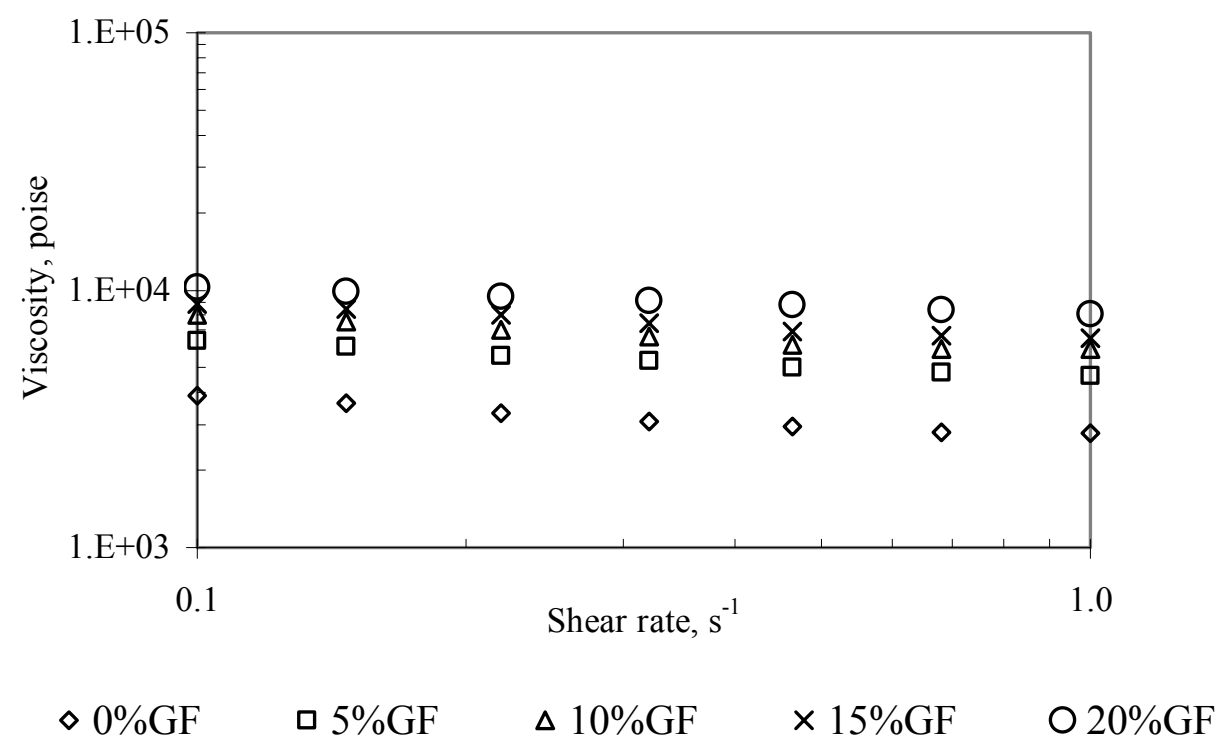

Figure 6-8 50\% virgin polycarbonate $+50 \%$ recycled polycarbonate steady-state viscosity at temperature $260{ }^{\circ} \mathrm{C}$

As seen in Figure 6-9, the viscosity of virgin unreinforced polycarbonate is much higher than the viscosity of recycled polycarbonate. This is due to degradation of the recycled polycarbonate and it is common for recycled polymer to have a lower viscosity level that the virgin polymer. This is consisting with melt flow index results which showed that recycled polycarbonate has lower molecular weight than virgin polycarbonate. When glass fiber is added to the recycled polycarbonate the difference in viscosities between glass reinforced virgin and glass reinforced recycled polycarbonate is reduced, as seen in Figure 6-10. 


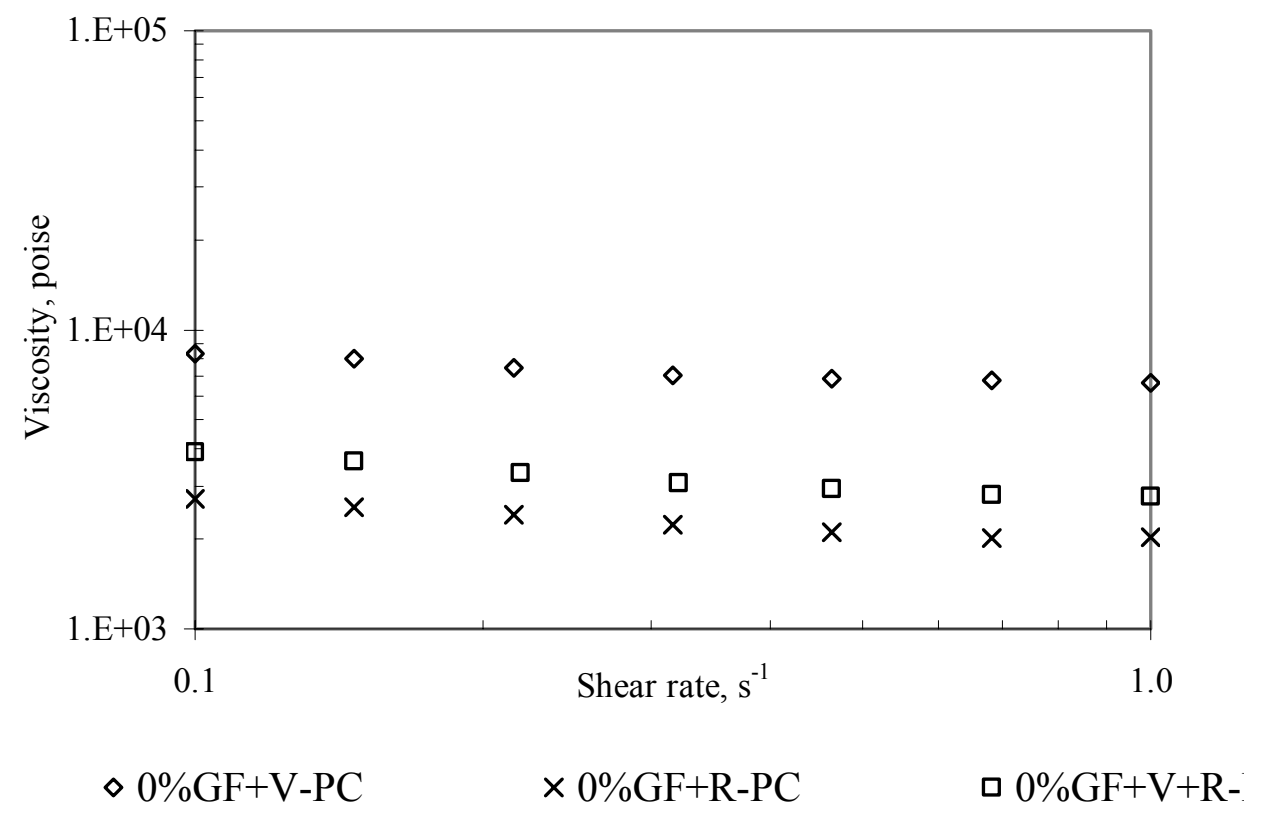

Figure 6-9 Unreinforced polycarbonate steady-state viscosity at temperature $260{ }^{\circ} \mathrm{C}$

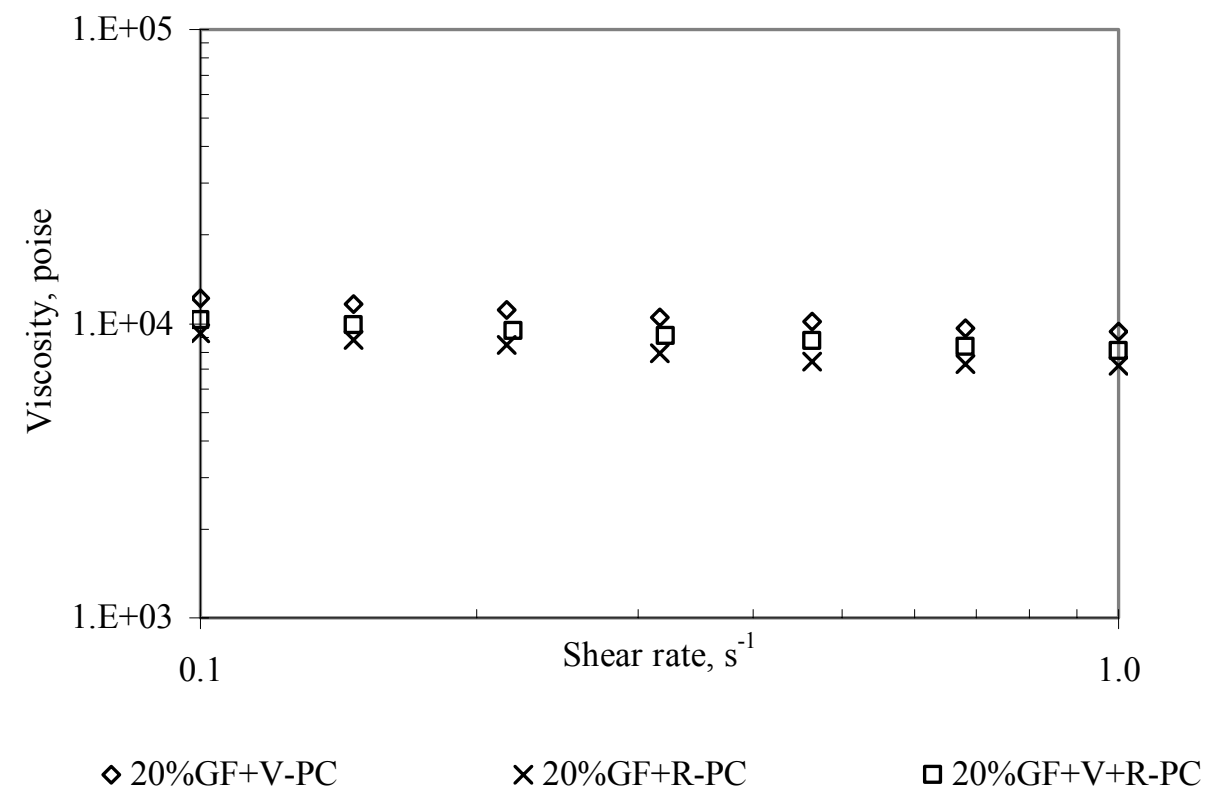

Figure 6-10 20\% glass reinforced PC steady-state viscosity at temperature $260{ }^{\circ} \mathrm{C}$ 
Figure 6-11 shows the relative viscosity, $\eta_{\mathrm{r}}=\frac{\eta_{\mathrm{c}}}{\eta_{\mathrm{m}}}$, where $\eta_{\mathrm{c}}$ is the composite viscosity and $\eta_{\mathrm{m}}$ is the unfilled polymer viscosity of the three systems at shear rate $1.0 \mathrm{~s}^{-1}$ and temperature equal to $260{ }^{\circ} \mathrm{C}$. It is seen that the relative viscosity of the increases with increasing the volume fraction of the glass fiber. The recycled polycarbonate has higher relative viscosity because the unfilled recycled polycarbonate has lower viscosity that the unfilled virgin polycarbonate.

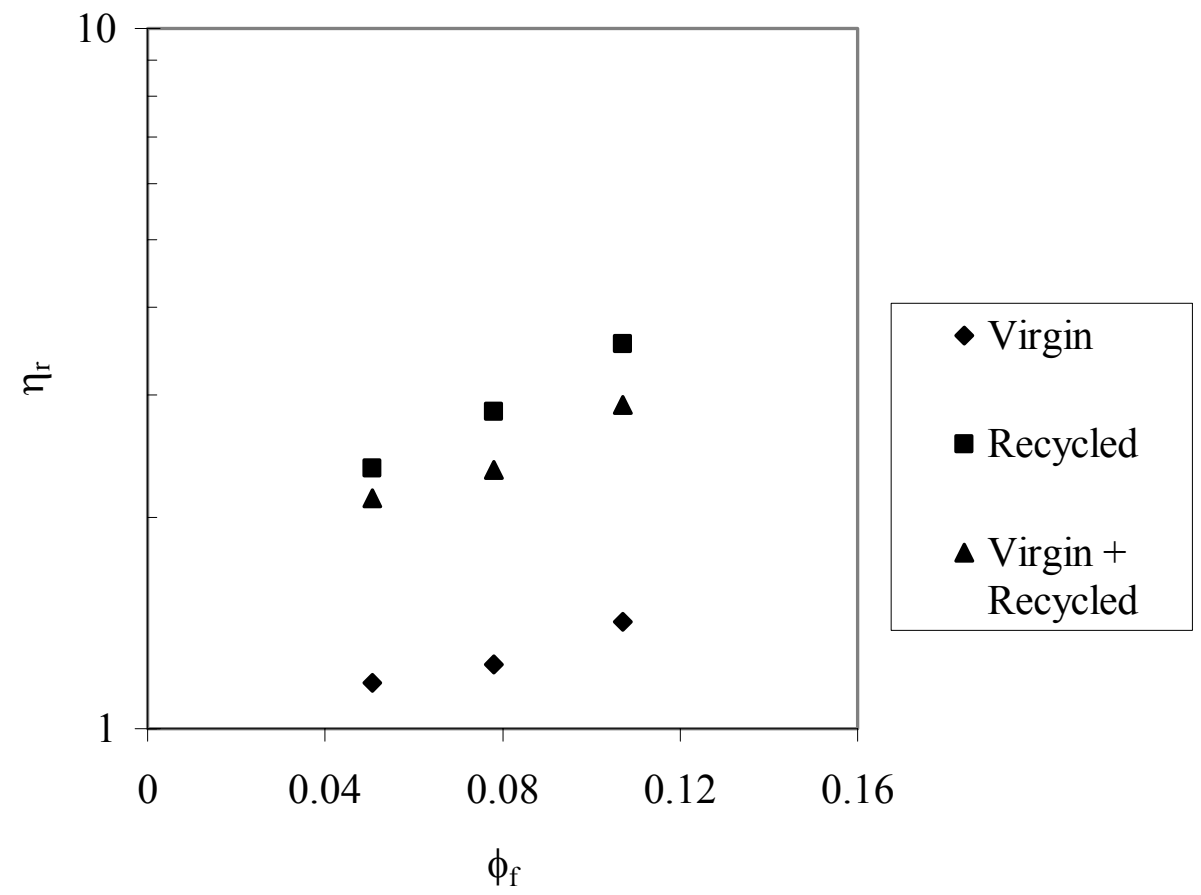

Figure 6-11 Variation of relative viscosity at constant shear rate with glass fiber volume fraction for all three systems 


\subsubsection{Carreau steady-state predictions}

The Carreau model is a three-parameter viscosity model that is sufficiently flexible to fit a wide variety of experimental viscosity curves. The model is:

$$
\frac{\eta-\eta_{\infty}}{\eta_{0}-\eta_{\infty}}=\frac{\eta}{\eta_{0}}=\left[1+(\lambda \dot{\gamma})^{2}\right]^{(\mathrm{n}-1) / 2}
$$

where $\dot{\gamma}$ is the shear rate, $\eta_{\mathrm{o}}$ is the zero-shear-rate viscosity, $\eta_{\infty}$ is the infinite-shear-rate viscosity and here set to zero, $\lambda$ is a time constant, and $n$ is the "power-law exponent" . The time constants are associated with the thermal and configuration changes taking place along the polymer chain as a response to an applied stress. The analytical expression for the non-Newtonian viscosity curve of the Carreau model is very useful for numerical calculation because it describes the viscosity over the entire shear-rate range, including the low shear rates. The coefficients for the Carreau model are determined from the steady-state viscosity data (see Table 6-3). Using the experimental data the parameters can be estimated for unfilled virgin polycarbonate, unfilled recycled polycarbonate, and unfilled 50\% mixture. The Carreau coefficients for the three polymers are given in Table 6-3. The viscosity for the unfilled polymer melts are given in Figures 6-12, 6-13, and 6-14. 
Table 6-3 Polymer Carreau coefficients

\begin{tabular}{llll}
\hline \hline Material & $\eta_{\mathbf{o}}$, poise & $\lambda$, sec & $\mathbf{n}$ \\
\hline Virgin PC & 7858 & 1.5 & 0.6 \\
& & & 0.6 \\
Recycled PC & 2535 & 1.9 & 0.6 \\
$50 \%$ Virgin PC $+50 \%$ Recycled PC & 3548 & 1.9 & \\
\hline \hline
\end{tabular}

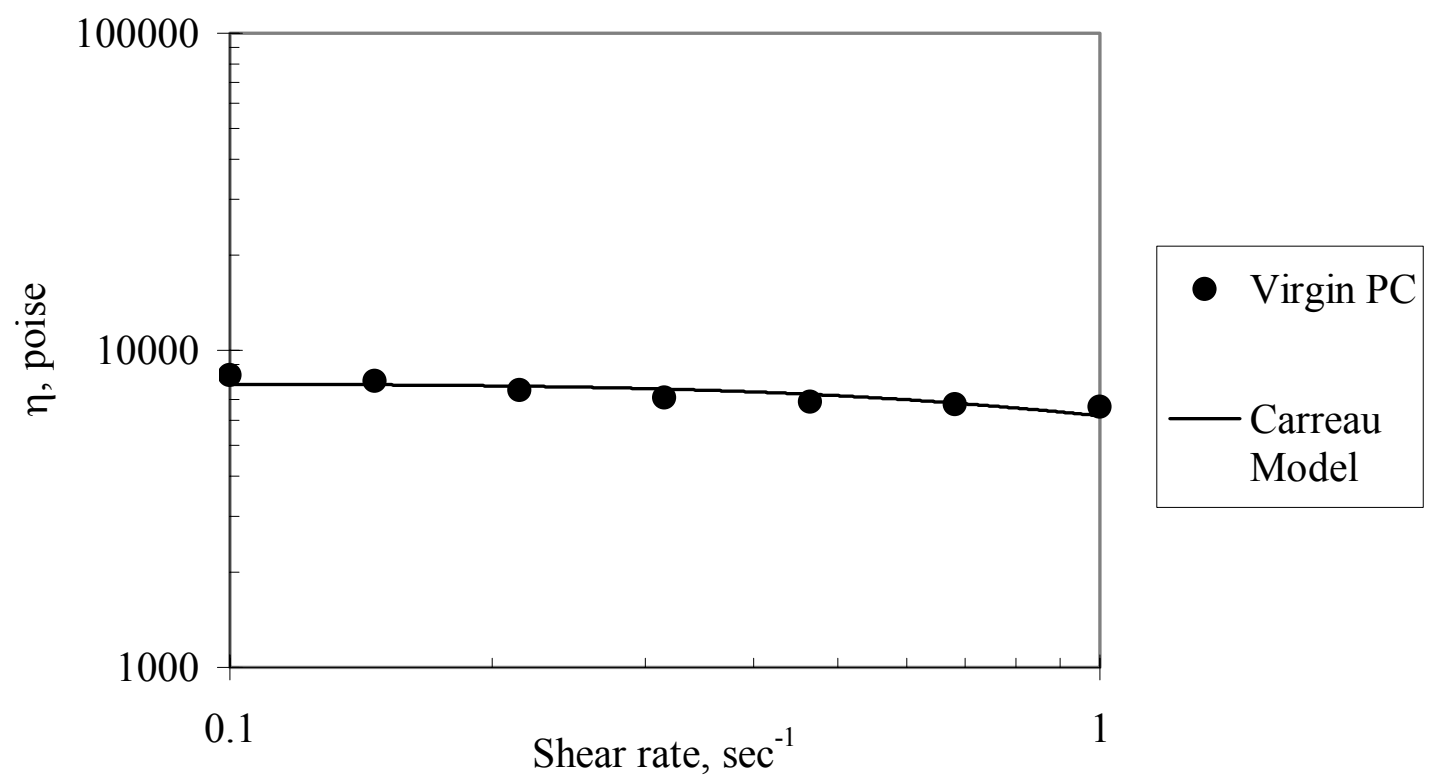

Figure 6-12 Virgin polycarbonate predicted steady state viscosity vs. shear rate using Carreau 


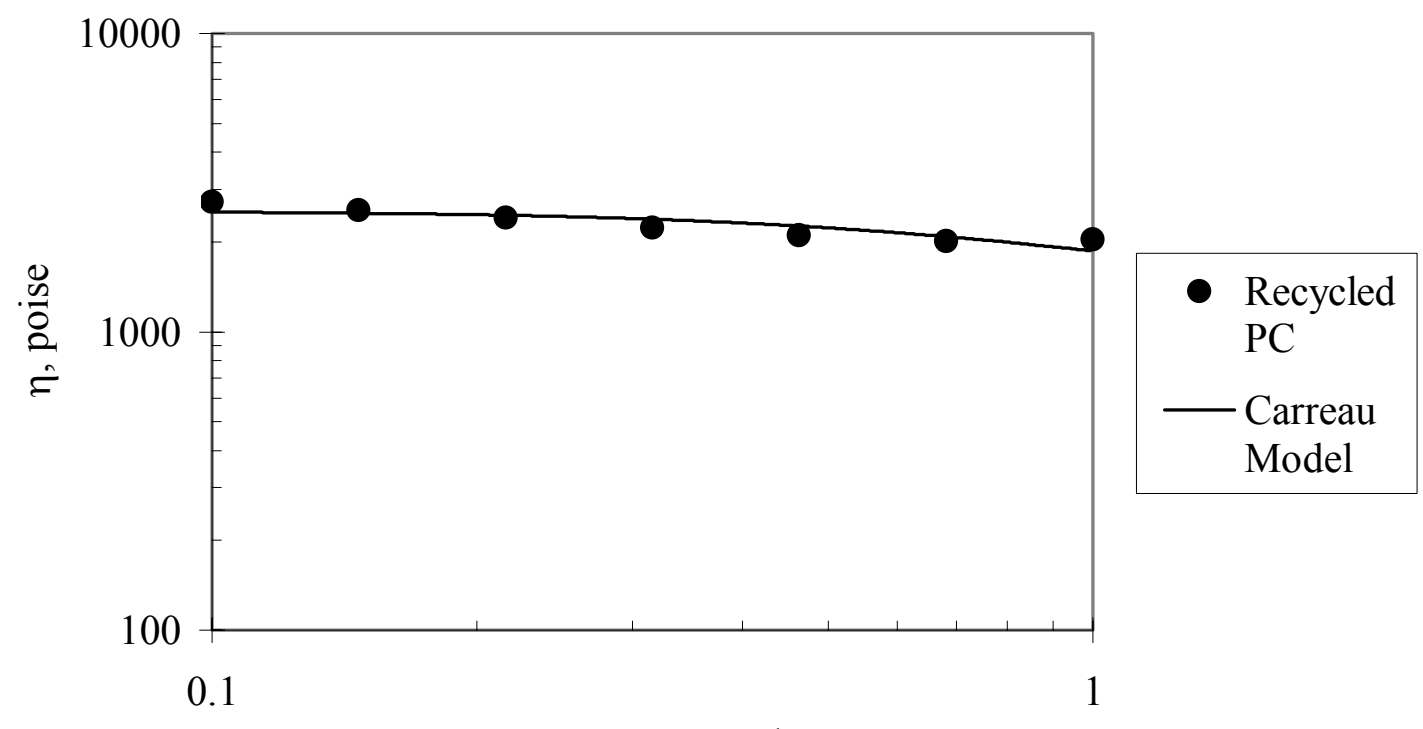

Shear rate, $\sec ^{-1}$

Figure 6-13 Recycled polycarbonate predicted steady state viscosity vs. shear rate using Carreau

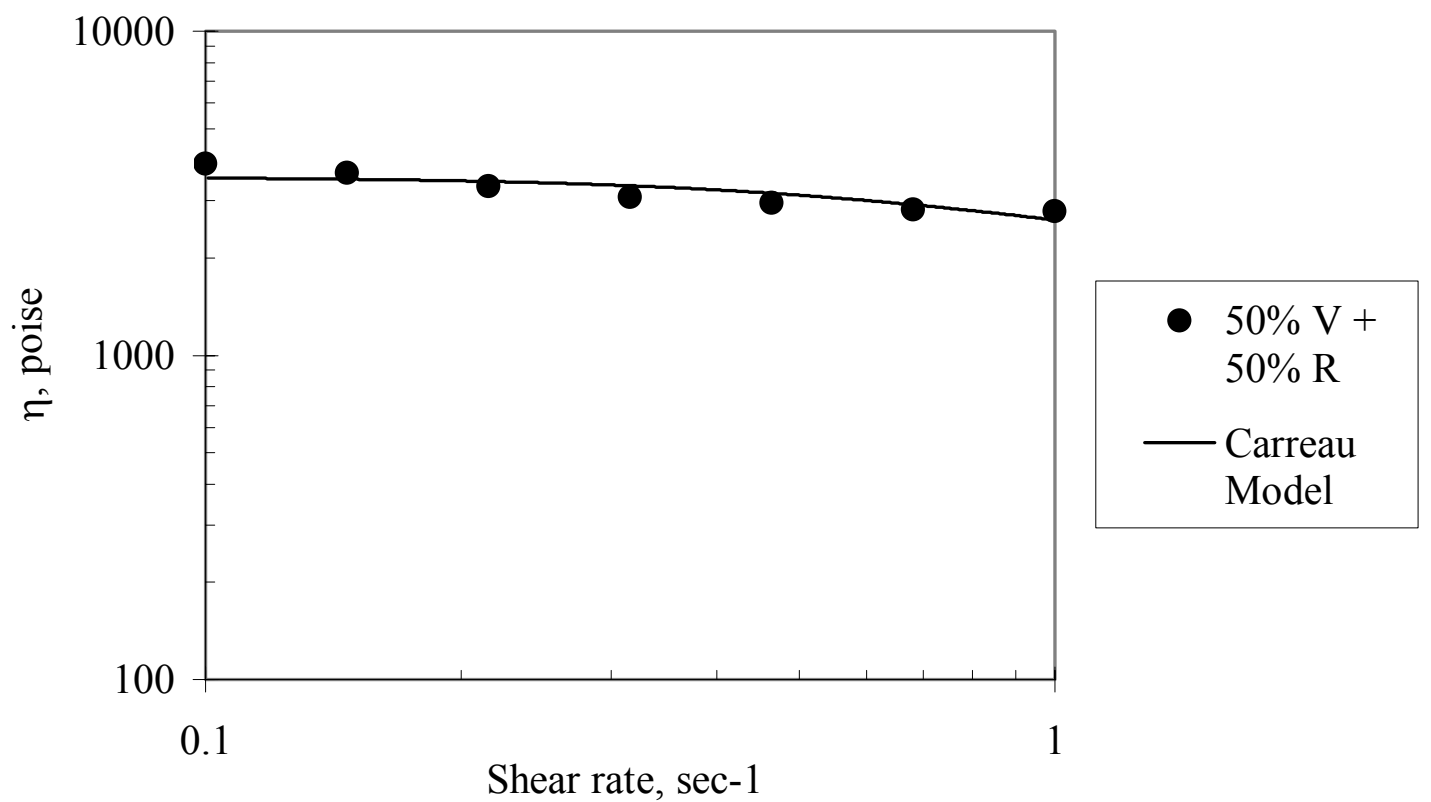

Figure 6-14 50\% virgin polycarbonate $+50 \%$ recycled polycarbonate predicted steady state viscosity vs. shear rate using Carreau 


\subsection{Dynamic testing results (samples are solid)}

DMA tests can be conducted in a variety of ways. For solid materials, the most common experiment is a temperature sweep. A frequency equal $1 \mathrm{~Hz}$ and $0.1 \%$ strain of amplitude are selected and maintained as constants throughout the experiment. A heating routine is selected and the material temperature is raised from the desired starting temperature $\left(40{ }^{\circ} \mathrm{C}\right)$ to an endpoint temperature $\left(160{ }^{\circ} \mathrm{C}\right)$. Figures $6-15$ through $6-20$ demonstrate the storage and loss modulus for all three systems. Both the storage and loss modului increase as the glass fiber content increases. The unreinforced virgin polycarbonate storage and loss modulus is higher than the storage and loss modulus of unreinforced recycled polycarbonate and this is consistent with the viscosity results presented earlier. The addition of glass fiber to virgin, recycled, and mixture of virgin and recycled polycarbonate the glass transition temperature did not change significantly (as seen in Figures 6-15 through 6-17).

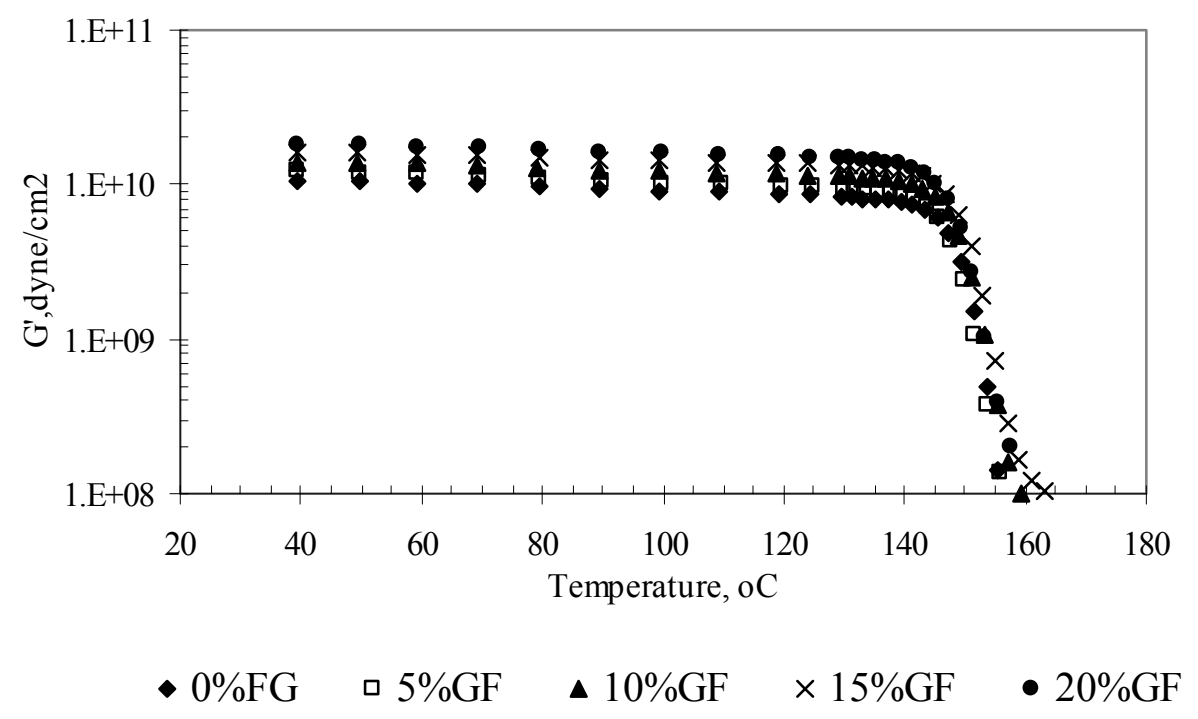

Figure 6-15 Storage modulus for unreinforced and glass reinforced virgin polycarbonate 


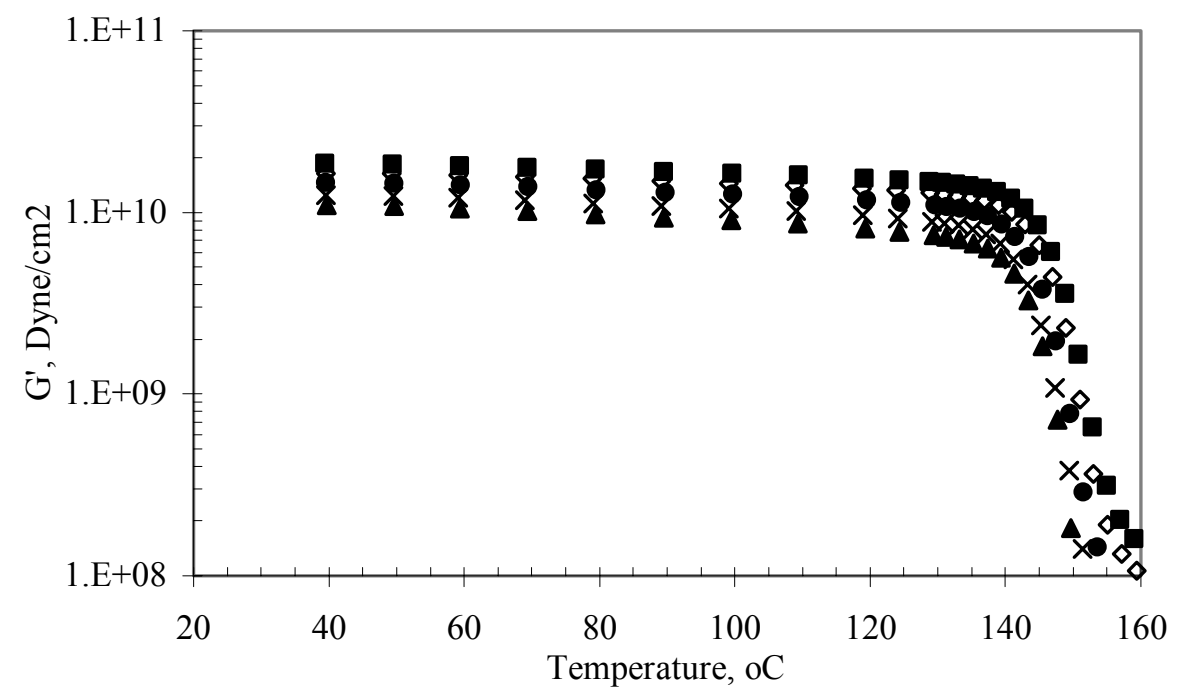
\ $0 \% \mathrm{GF}$
$\times 5 \% \mathrm{GF}$
- $10 \% \mathrm{GF}$
$\diamond 15 \% \mathrm{GF}$
- $20 \% \mathrm{GF}$

Figure 6-16 Storage modulus for unreinforced and glass reinforced recycled polycarbonate

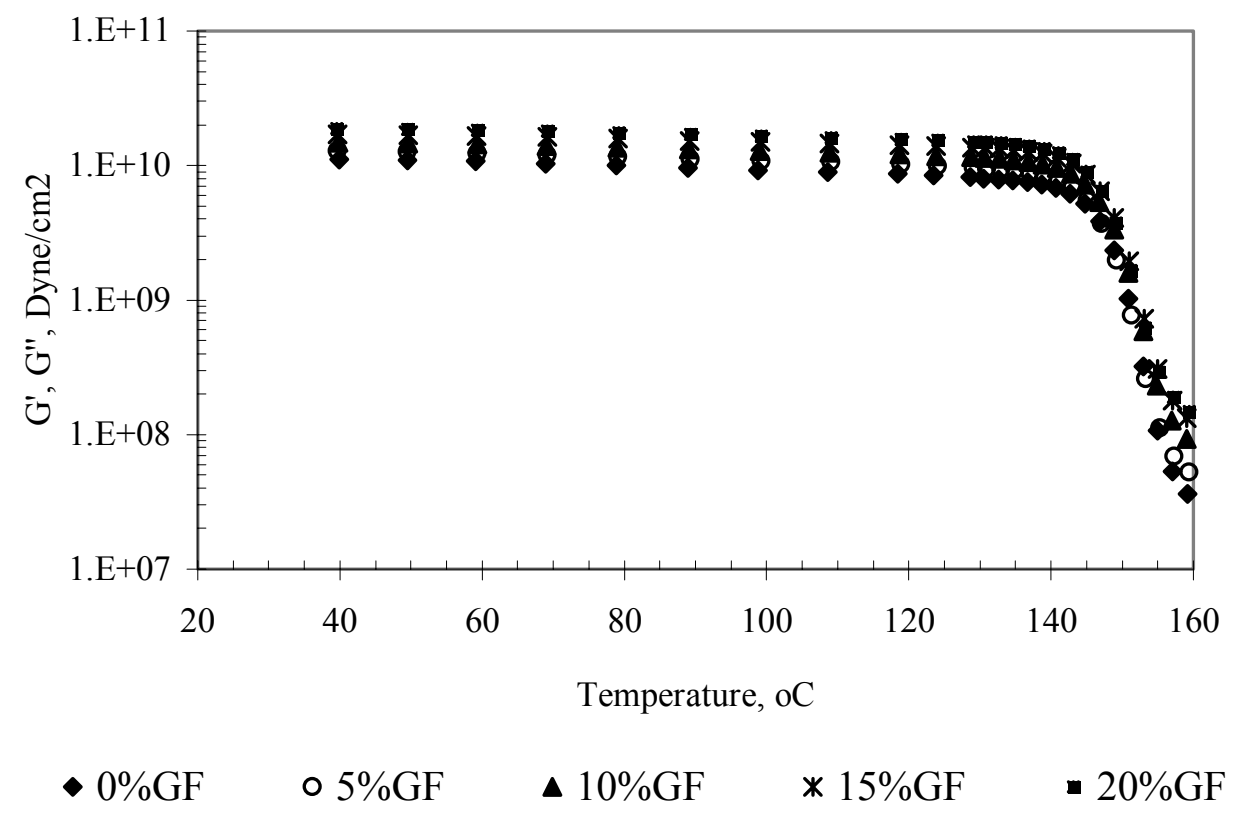

Figure 6-17 Storage modulus for unreinforced and glass reinforced 50\% virgin and 50\% recycled polycarbonate 


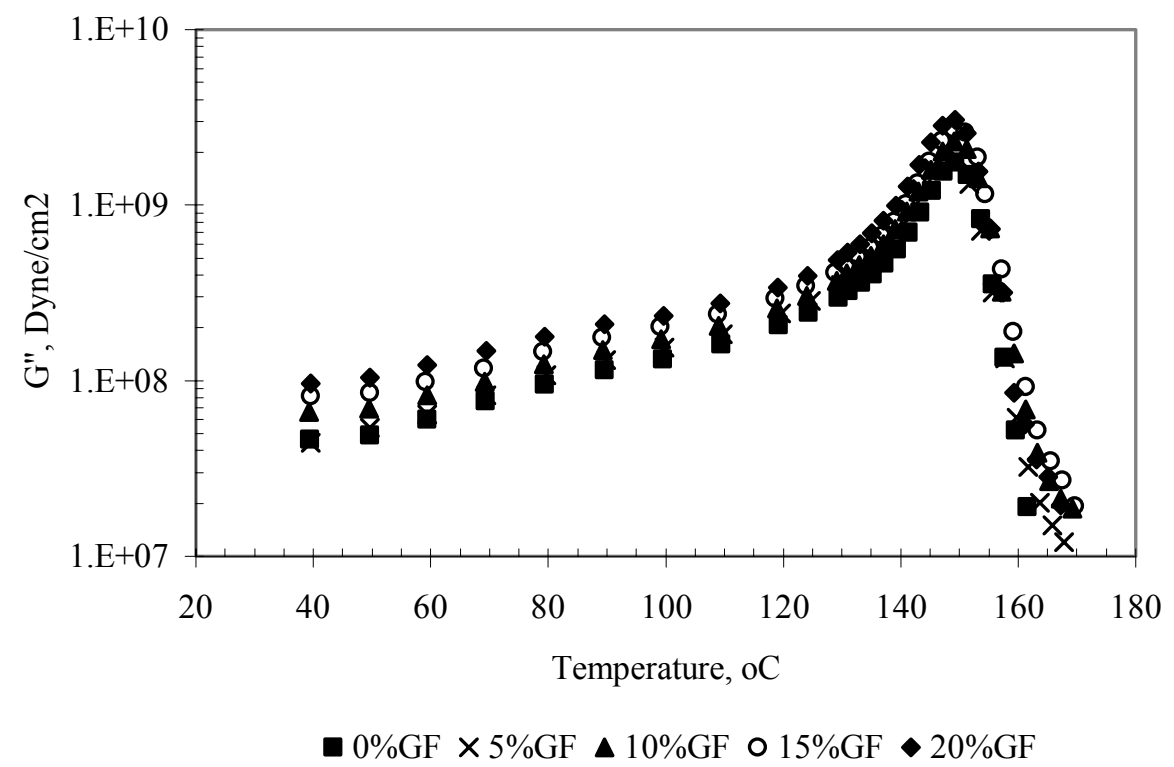

Figure 6-18 Loss modulus for unreinforced and glass reinforced virgin polycarbonate

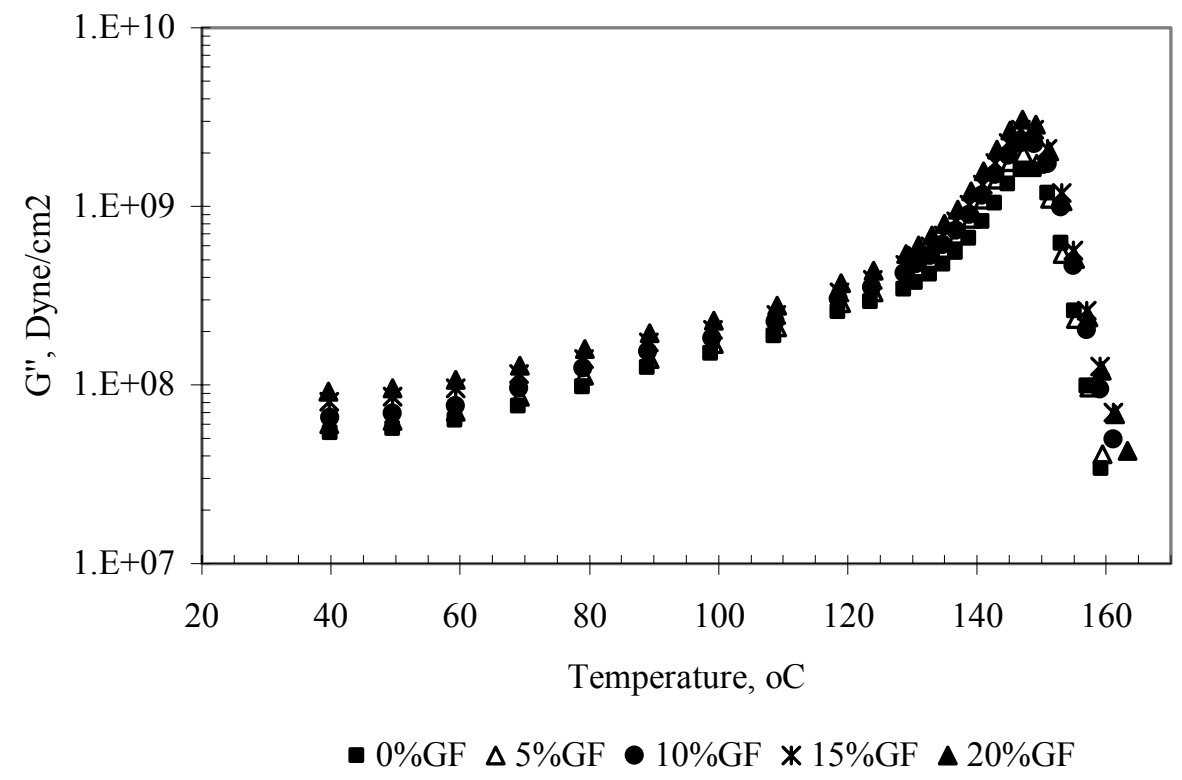

Figure 6-19 Loss modulus for unreinforced and glass reinforced recycled polycarbonate 


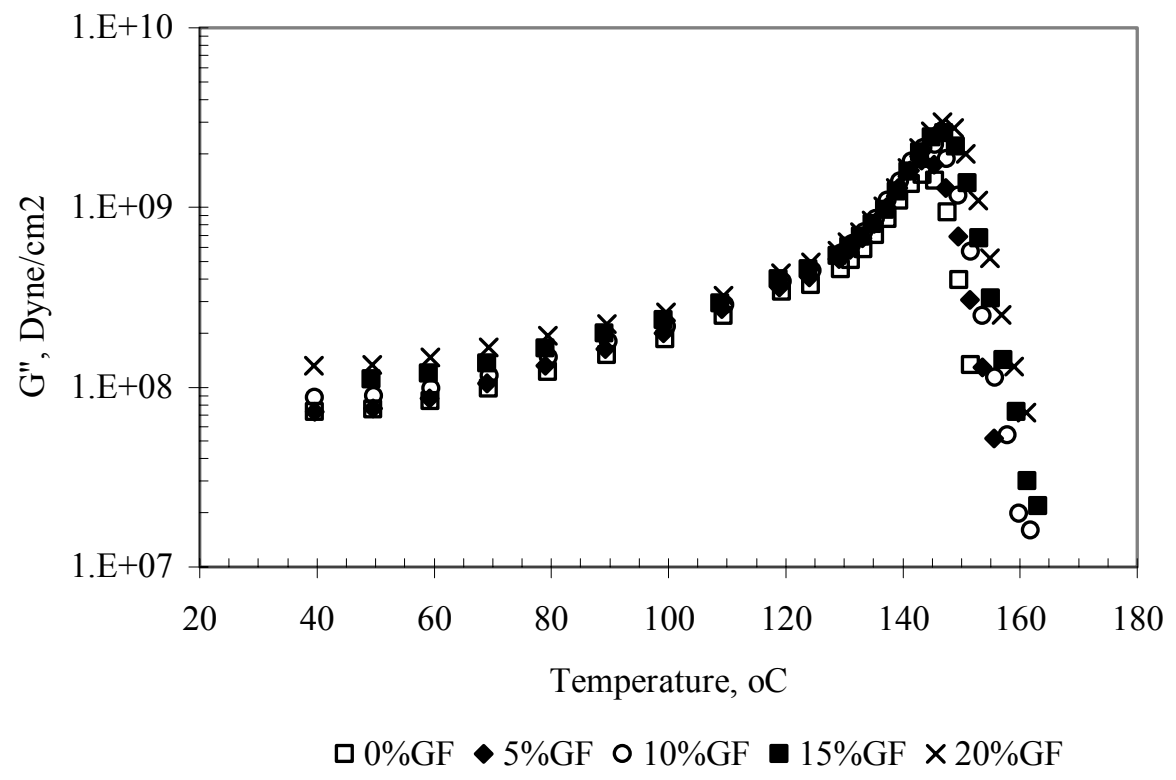

Figure 6-20 Loss modulus for unreinforced and glass reinforced 50\% virgin and 50\% recycled polycarbonate

The storage modulus of unreinforced recycled polycarbonate is less than the storage modulus of unreinforced virgin polycarbonate (as seen in Figure 6-21). When the glass fiber is added to the virgin and recycled polycarbonate the difference is reduced as seen in Figures 6-22. In the case of the loss modulus, unreinforced recycled polycarbonate has higher loss modulus than unreinforced virgin polycarbonate (as seen in Figure 6-23). The addition of glass fiber decreases the difference of loss modulus of recycled and virgin polycarbonate and this is consistent with the viscosity results presented earlier (see Figure 6-24). 


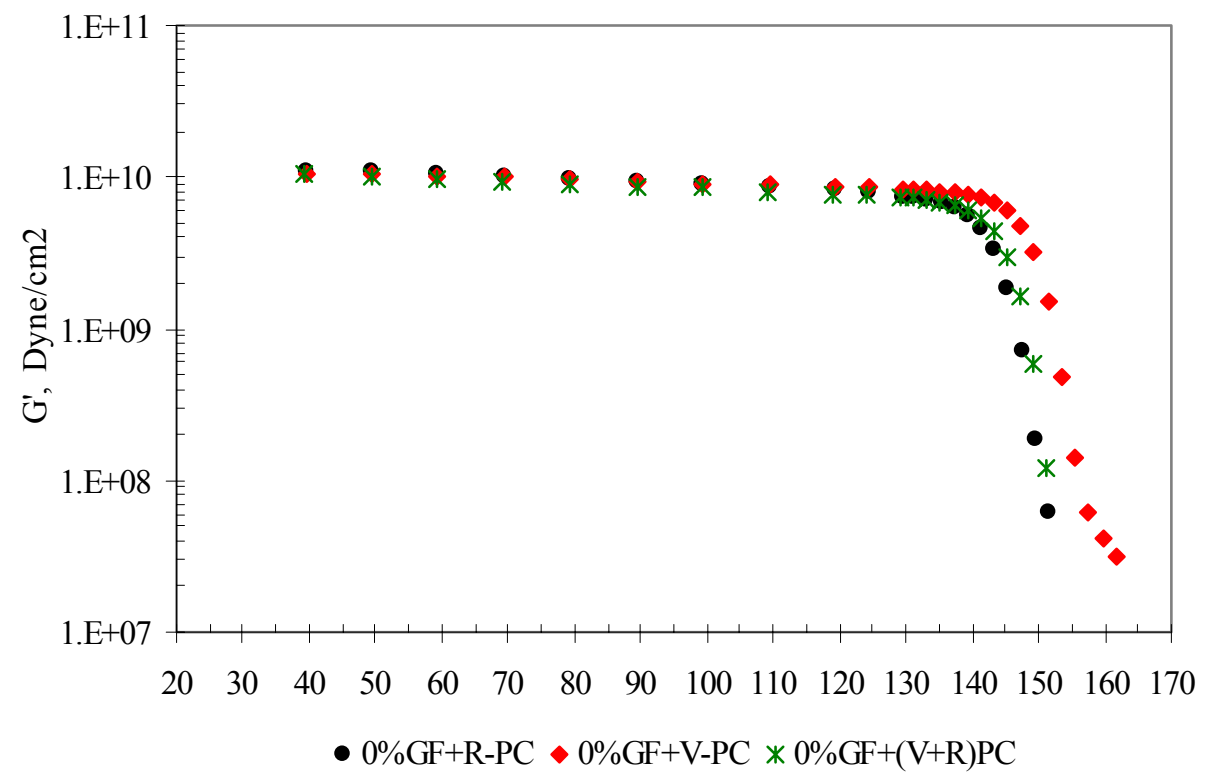

Figure 6-21 Storage modulus for unreinforced polycarbonate vs. temperature

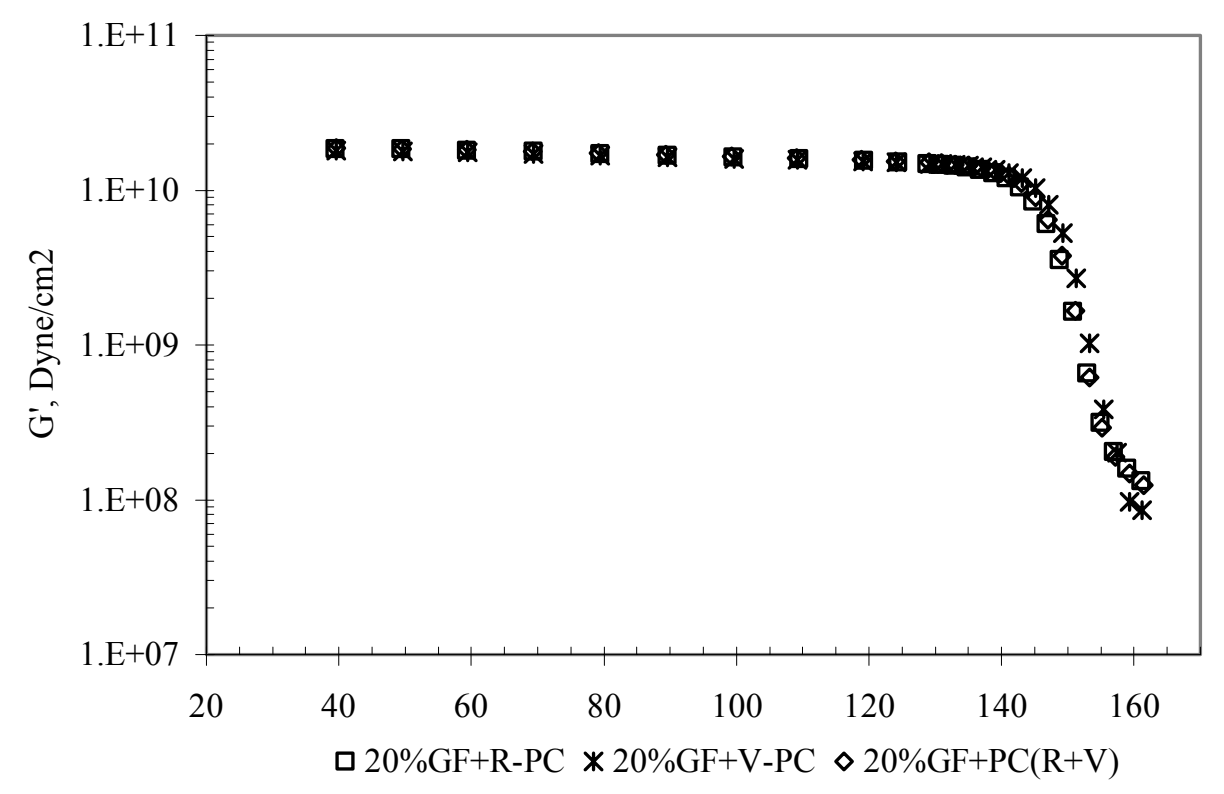

Figure 6-22 Storage modulus for 20\% glass reinforced polycarbonate vs. temperature 


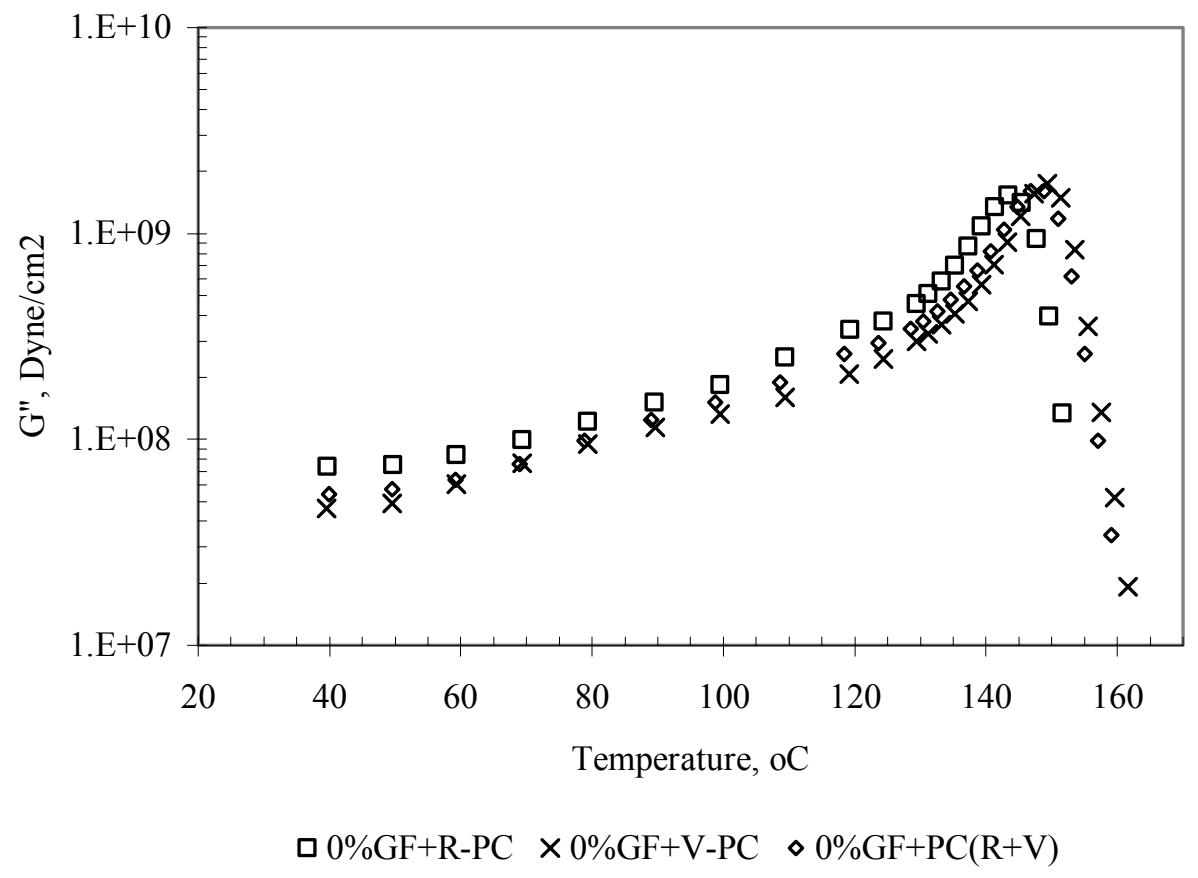

Figure 6-23 Loss modulus for unreinforced polycarbonate vs. temperature

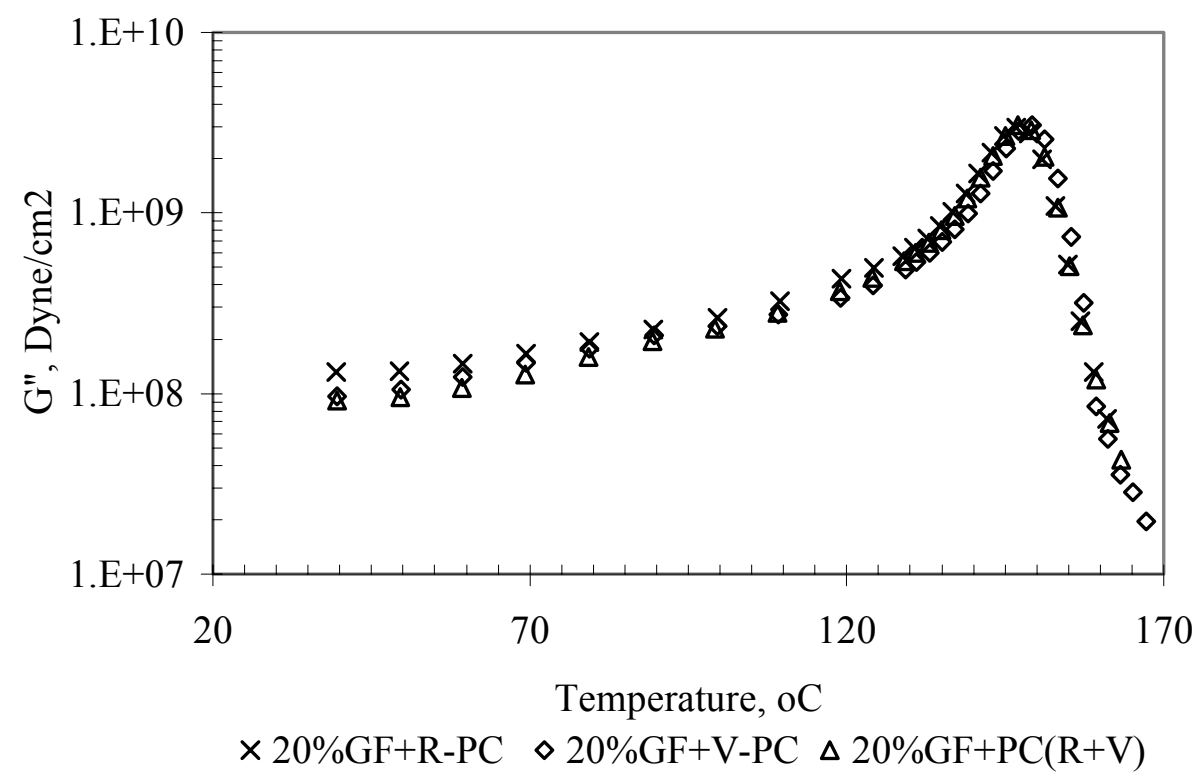

Figure 6-24 Loss modulus for 20\% glass reinforced polycarbonate vs. temperature 


\subsection{Coefficient of thermal expansion}

In most materials, an increase in temperature will result in an increase in the dimensions. Given a bar of length $1_{0}$ that is subjected to a one-degree rise in temperature, the linear thermal expansion coefficient, $\alpha$, is defined as

$$
\alpha=\frac{\Delta 1}{1_{\mathrm{o}}}
$$

where $\Delta \mathrm{l}$ is the change in length associated with the unit temperature rise.

The unit for the coefficient of linear expansion is $\mathrm{cm} / \mathrm{cm} /{ }^{\circ} \mathrm{C}$. As shown in Figure 6-25 the thermal expansion of unfilled material is higher than the thermal expansion of filled material. Results indicate that the thermal expansion decreases as the amount of fibers increases giving the material more dimensional stability. One of the main reasons of adding glass fiber to polymer is to get the dimensional stability. When glass fiber was added to recycled polycarbonate the thermal expansion decreases in the same way when the glass fiber is been added to virgin PC as seen in Figure 6-25. It is also evident from Figure $6-25$, that the variation of the thermal of expansion with respect glass fiber wt $\%$ is reasonably linear suggesting that the behavior follows some form of the rule of mixtures. 


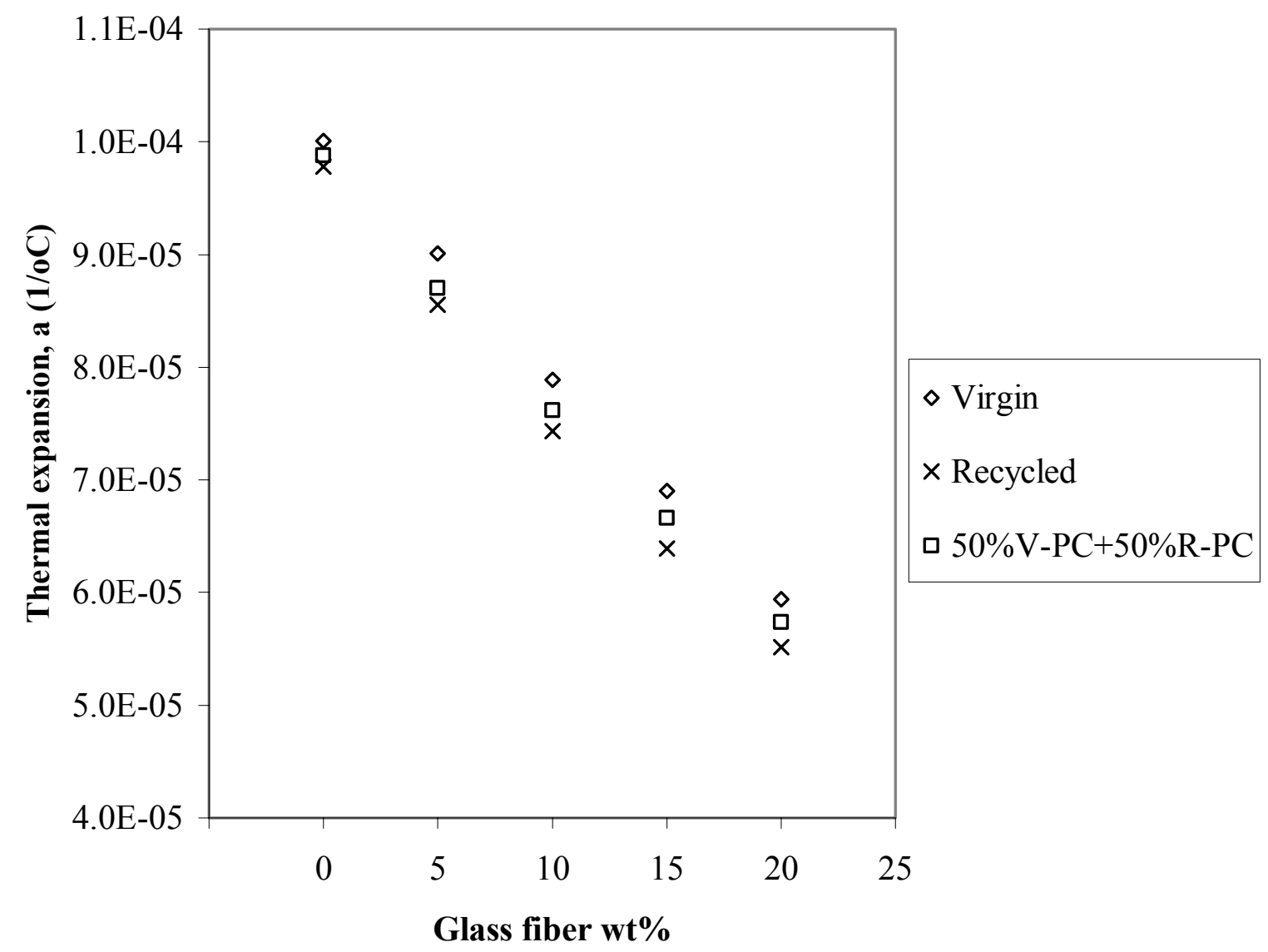

Figure 6-25 Effect of glass fiber on the coefficient of thermal of expansion

\subsection{Dynamic testing results (samples are molten)}

As in the case of solids, a polymer melt can be subjected to an oscillatory-shear experiment to study the time-dependent behavior of the polymer. The testing involves the rotation of the upper plate in a small-amplitude sinusoidal oscillation at a frequency, $\omega$. The instantaneous velocity will be nearly linear between the parallel plates if the distance between the plates, $\mathrm{h}$, is small enough to insure that the quantity, $\omega \rho^{2} / \eta_{\mathrm{o}}$ is much less than unity. The other two constants are the density, $\rho$, and the viscosity at zero shear rate, 
$\eta_{\mathrm{o}}$. The shear rate is independent of position since the velocity profile is linear between the plates, and is given as:

$$
\dot{\gamma}(\mathrm{t})=\dot{\gamma}^{\mathrm{o}} \cos \omega \mathrm{t}
$$

The strain of the material is by definition the integral of the shear rate between times 0 and t:

$$
\gamma(0, \mathrm{t})=\gamma^{\mathrm{o}} \sin \omega \mathrm{t}
$$

where $\gamma^{\mathrm{o}}$ and $\dot{\gamma}^{\mathrm{o}}$ are the positive amplitude of the shear-strain and shear-rate oscillations. The shear stress can be defined in terms of viscoelastic-material functions, G', G' as in:

$$
\tau=-\mathrm{G}^{\prime}(\omega) \gamma^{\mathrm{o}} \sin \omega \mathrm{t}-\mathrm{G}^{\prime \prime} \gamma^{\mathrm{o}} \cos \omega \mathrm{t}
$$

Here, the material functions are the storage modulus, G', the loss modulus, G'. The storage modulus, G' gives information about the elastic nature of the polymer. Conversely, the loss modulus, G", is gives information about the viscous or energy dissipation during flow. The tangent of the phase angle between the stress and strain is referred to as the loss tangent, $\tan \delta$.

The Rheometric Mechanical Spectrometer (RMS-800) was used to measure dynamic properties of the polymers. The storage modulus, and loss modulus were measured using a parallel-plate viscometer. The upper disk was oscillated at an angular velocity $(\omega)$ and the lower disk was fixed. The parallel-plate apparatus was used instead of the cone and plate apparatus because the length of the fiber was greater than the gap in the cone and plate. The gap between the parallel plates was greater than the length of the 
fiber (gap/length ratio $>1.0$ ), so that the fiber was not constrained by the walls. The parallel plate's diameter was $25 \mathrm{~mm}$ and the gap length was $1 \mathrm{~mm}$. The testing involved the rotation of the upper plate in a small-amplitude (strain 10\%) sinusoidal oscillation at an angular frequency sweep between 0.1 and $100 \mathrm{rad} / \mathrm{sec}$. All samples were dried at least 12 hours at temperature equal $120{ }^{\circ} \mathrm{C}$ before conducting the dynamic tests. The test procedure was:

- Calibrate the zero-gap plate distance at temperature $290{ }^{\circ} \mathrm{C}$

- Heat the sample to temperature equal $290{ }^{\circ} \mathrm{C}$

- Set the gap distance to $1 \mathrm{~mm}$

- Remove any extraneous material

- Start oscillating the upper plate

- Vary the frequency from 1 to $100 \mathrm{rad} / \mathrm{sec}$

- Record measurables using data acquisition hardware and software

- Repeating the above steps at temperatures $250{ }^{\circ} \mathrm{C}$ and $260{ }^{\circ} \mathrm{C}$.

The first step in performing the dynamic test was to ensure that the material would not degrade during the test period. To ensure there is no degradation time, sweep tests were performed. The time sweep test measures the dynamic properties such as the complex viscosity as a function of time at a constant frequency and strain. To obtain the linear region of the dynamic properties, a strain sweep test was conducted at a constant frequency as seen in Figure 6-26. All materials behaved linearly at a strain equal to $10 \%$. All dynamic tests in the following sections were conducted at a strain equal to $10 \%$. 


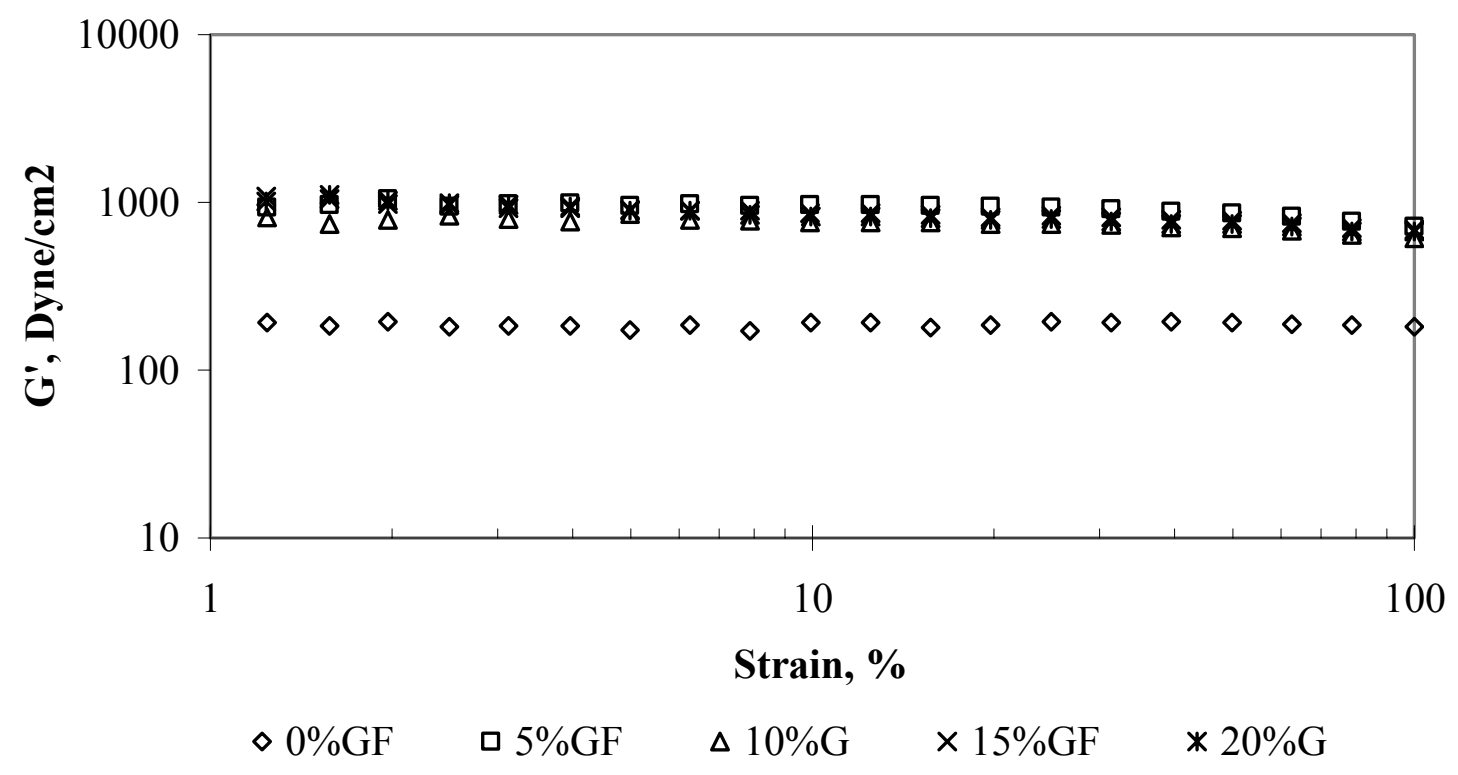

Figure 6-26 Strain sweep for virgin polycarbonate at frequency equal $1 \mathrm{~Hz}$ and temperature $250{ }^{\circ} \mathrm{C}$

\subsubsection{Storage modulus}

The linear-viscoelastic data for the three unfilled and filled materials are given in Figures 6-27, 6-28, and 6-29. The storage modulus indicates the viscous nature of the polymer and is affected by fibers in a similar fashion to the steady shear rate viscosity. As the fiber concentration increases, the storage modulus increases for all three systems. Figure 6-30 indicates that the storage modulus of unfilled virgin polycarbonate is less than the storage modulus of the recycled polycarbonate. This difference decreases when glass fiber is added to the recycled polycarbonate, as shown in Figure 6-31. The same behavior was observed for the storage modulus at temperature $250{ }^{\circ} \mathrm{C}$ and $260{ }^{\circ} \mathrm{C}$. The 
results for storage modulus at temperatures $250{ }^{\circ} \mathrm{C}$ and $260{ }^{\circ} \mathrm{C}$ are given in Appendix D. It is seen from Figure 6-32 that the storage modulus decreases as the temperature increases.

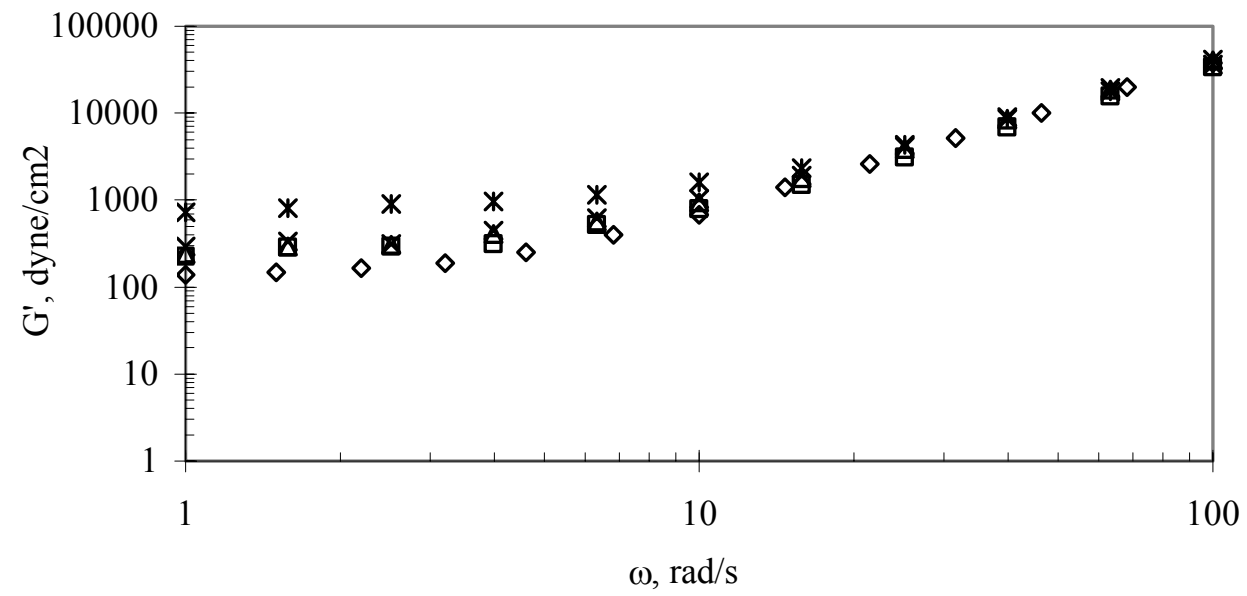

$\diamond 0 \% \mathrm{GF}+\mathrm{V}-\mathrm{PC}$ 口 $5 \% \mathrm{GF}+\mathrm{V}-\mathrm{PC} \Delta 10 \% \mathrm{GF}+\mathrm{V}-\mathrm{PC} \times 15 \% \mathrm{GF}+\mathrm{V}-\mathrm{PC} * 20 \% \mathrm{GF}+\mathrm{V}-\mathrm{PC}$

Figure 6-27 Virgin polycarbonate storage modulus vs. frequency $\left(\mathrm{T}=290{ }^{\circ} \mathrm{C}\right)$

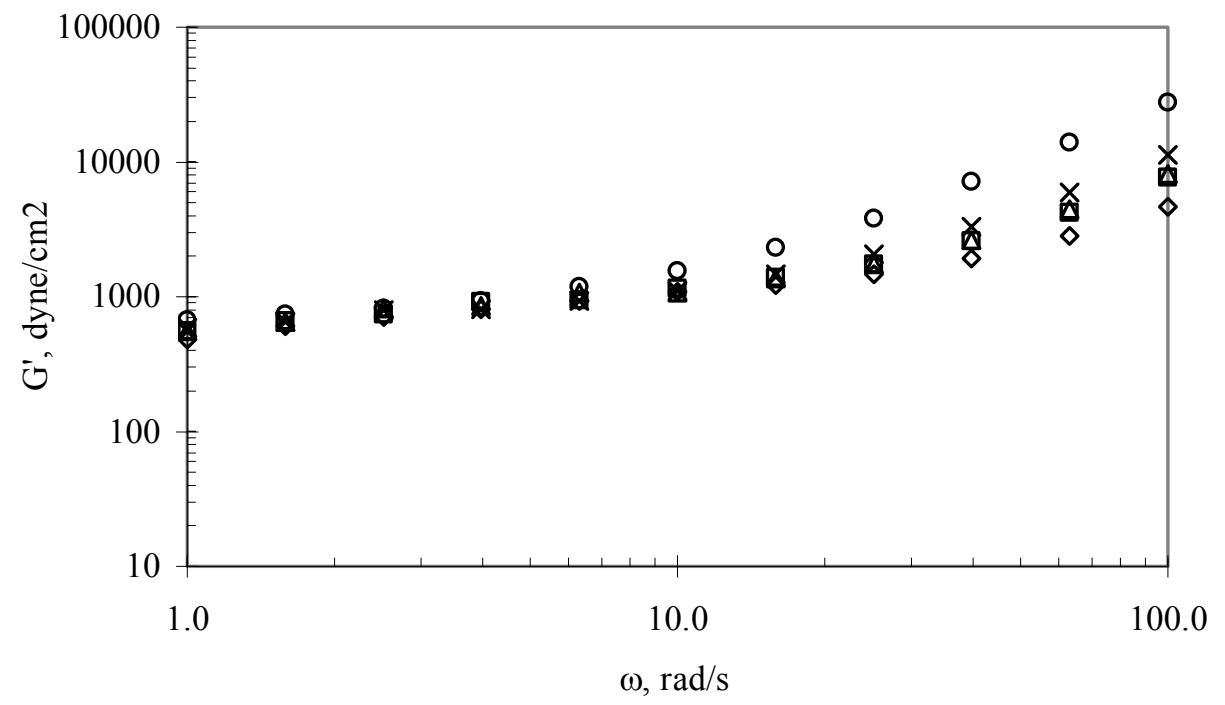

$\diamond 0 \% \mathrm{GF}+\mathrm{R}-\mathrm{PC} \quad \square 5 \% \mathrm{GF}+\mathrm{R}-\mathrm{PC} \quad \Delta 10 \% \mathrm{GF}+\mathrm{R}-\mathrm{PC} \quad \times 15 \% \mathrm{GF}+\mathrm{R}-\mathrm{PC} \quad \circ 20 \% \mathrm{GF}+$

Figure 6-28 Recycled polycarbonate storage modulus vs. frequency $\left(\mathrm{T}=290{ }^{\circ} \mathrm{C}\right)$ 


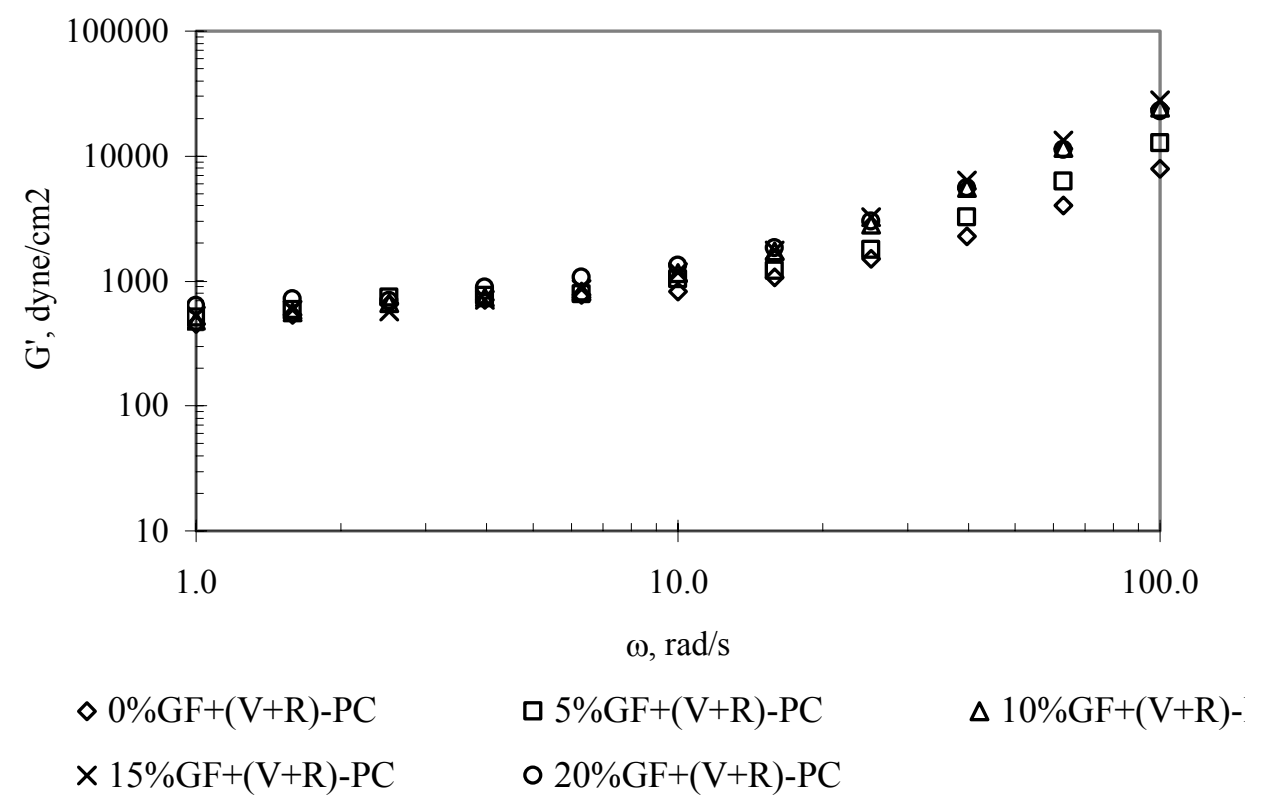

Figure $6-2950 \%$ virgin polycarbonate $+50 \%$ recycled polycarbonate storage modulus vs. frequency $\left(\mathrm{T}=290{ }^{\circ} \mathrm{C}\right)$

Figure 6-30 shows that there were large difference in the storage modulus of the ureinforced recycled and ureinforced virgin polycarbonate. This is consistent with the solid dynamic results presented earlier. When glass fiber is added to the recycled polycarbonate the difference in storage between glass reinforced virgin and glass reinforced recycled polycarbonate is reduced, as seen in Figure 6-31. 


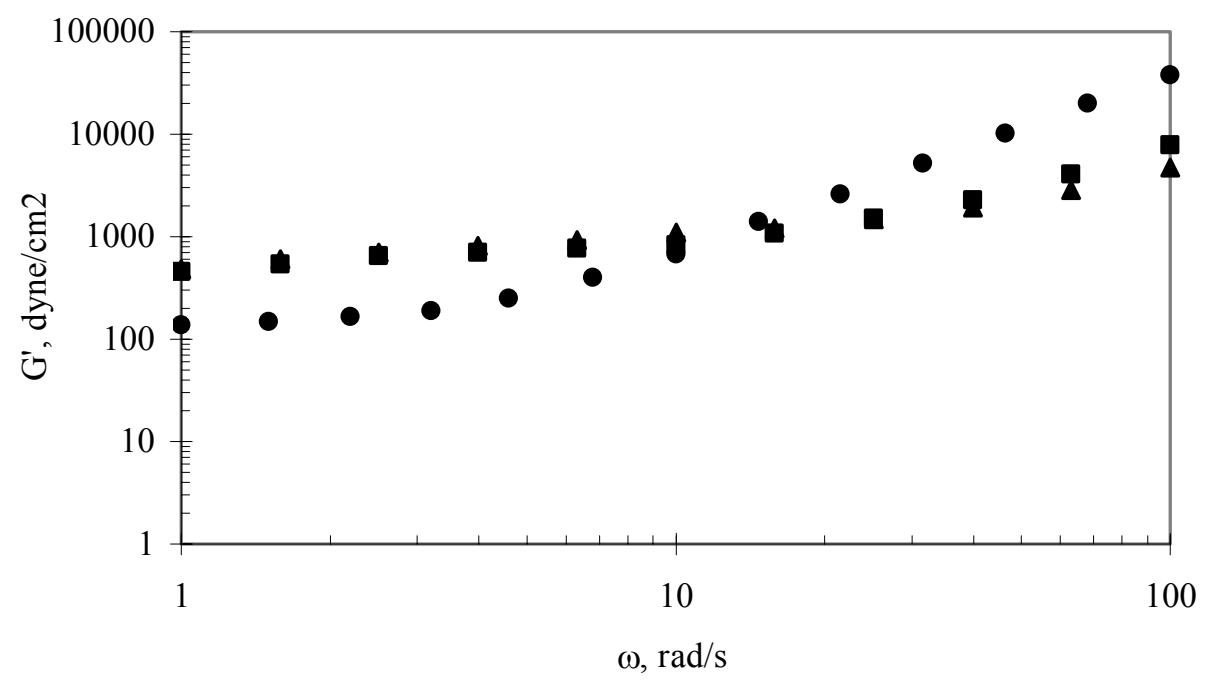

- $0 \% \mathrm{GF}+\mathrm{V}-\mathrm{PC} \quad \Delta 0 \% \mathrm{GF}+\mathrm{R}-\mathrm{PC} \quad$ - $0 \% \mathrm{GF}+(\mathrm{V}+\mathrm{R})-\mathrm{PC}$

Figure 6-30 Unfilled polycarbonate storage modulus vs. frequency $\left(\mathrm{T}=290{ }^{\circ} \mathrm{C}\right)$

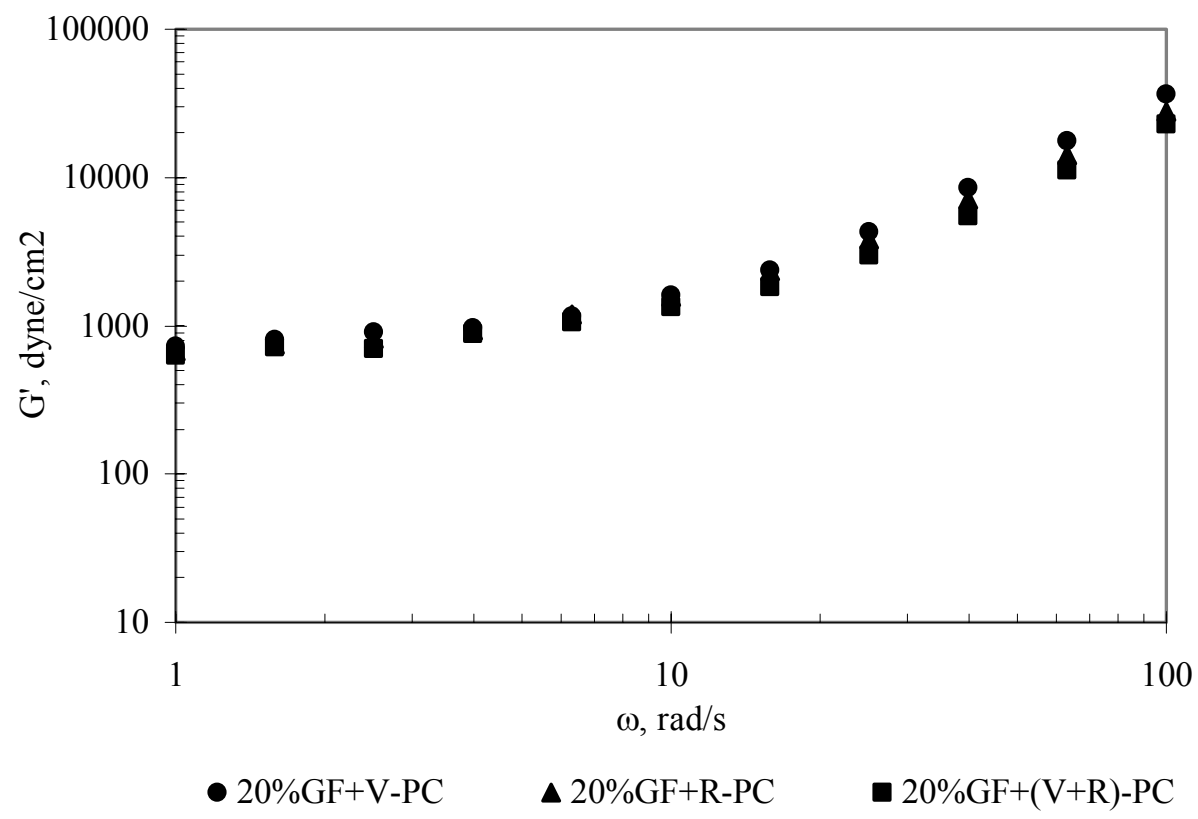

Figure 6-31 20\% glass fiber reinforced polycarbonate storage modulus vs. frequency $\left(\mathrm{T}=290{ }^{\circ} \mathrm{C}\right)$ 
It is seen from Figure 6-32 that the storage modulus decreases as the temperature increases. At higher temperature the polymer becomes more liquid like.

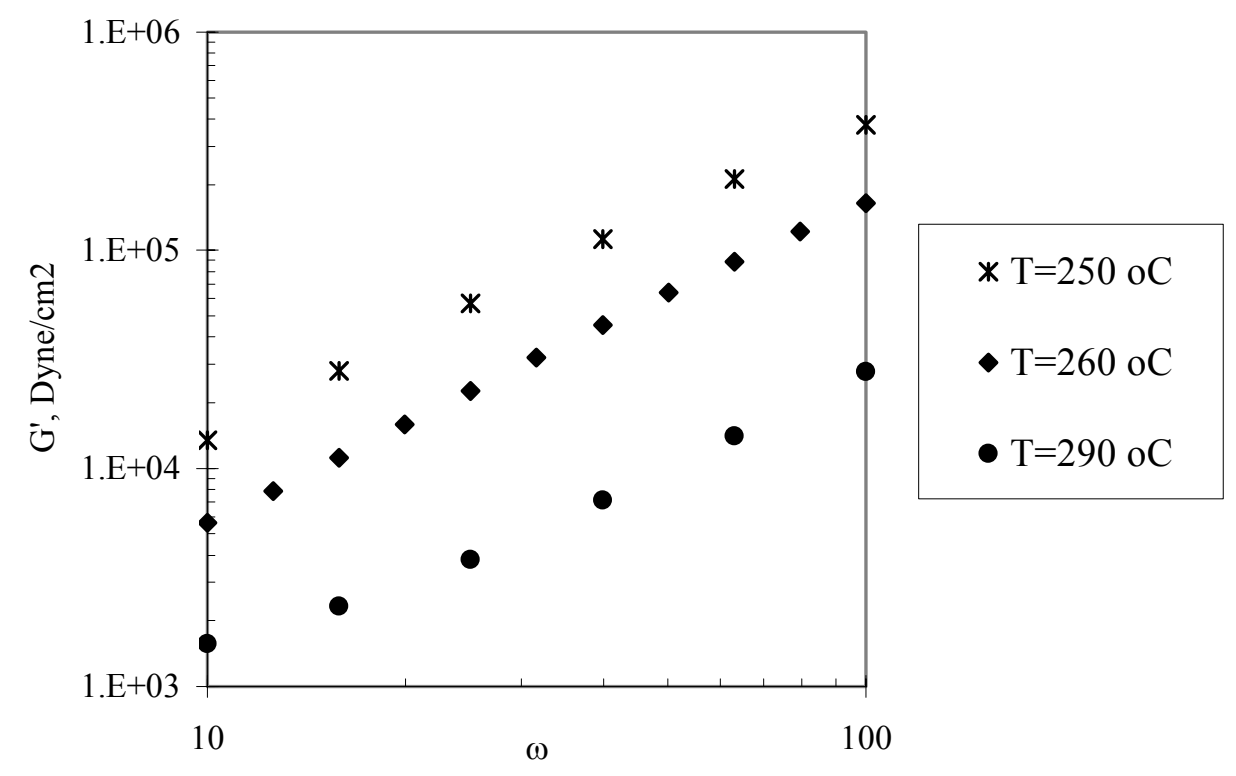

Figure 6-32 Influence of temperature on the storage modulus of $20 \%$ glass reinforced recycled PC

\subsubsection{Loss modulus}

The loss modulus indicates the viscous nature of the polymer. The loss modulus, G”, gives information about the energy dissipation during flow. The fibers will affect the loss modulus in a similar fashion as the storage modulus as seen in Figures 5-33, 5-34, and 5-35. The fibers will increase the loss modulus and will reduce the difference in loss modulus of virgin polycarbonate and recycled polycarbonate as seen in Figures 5-36 and 5-37. It is seen from Figure 6-38 that the loss modulus decreases as the temperature increases. 


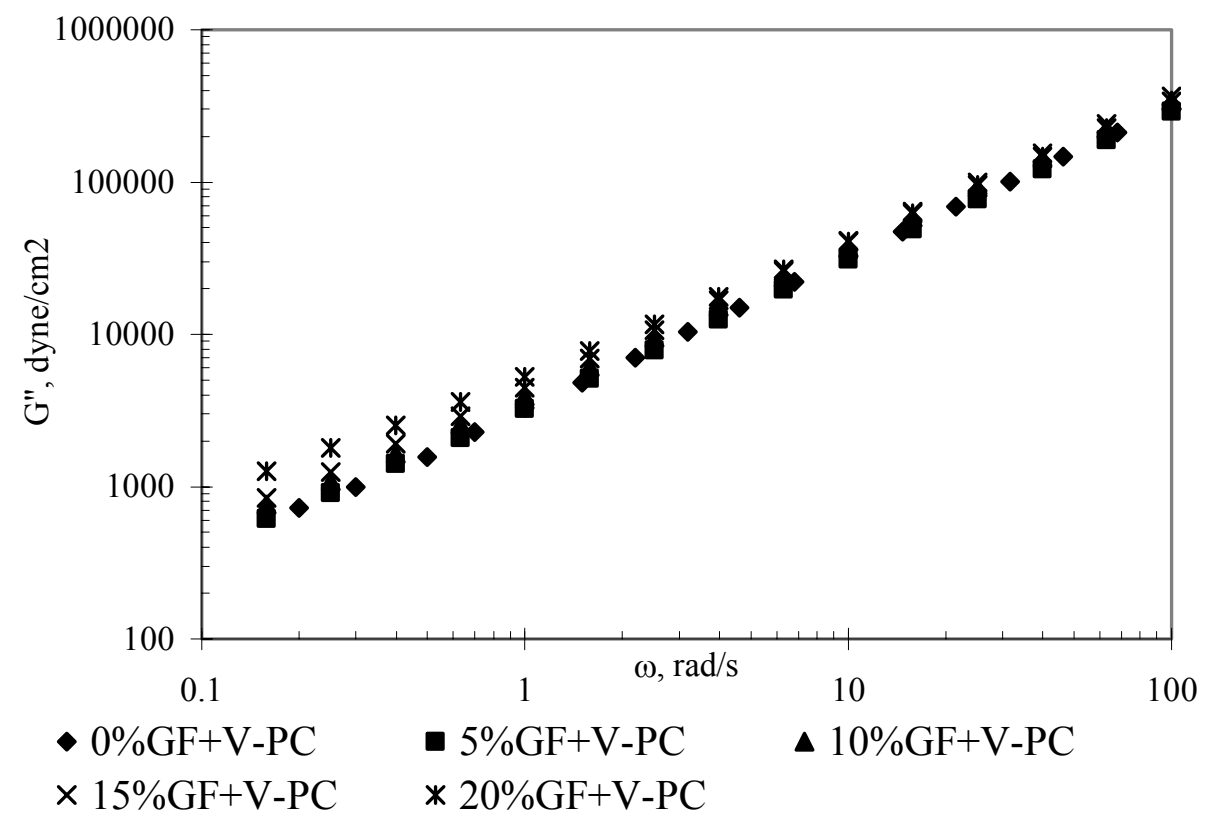

Figure 6-33 Virgin polycarbonate loss modulus vs. frequency $\left(\mathrm{T}=290{ }^{\circ} \mathrm{C}\right)$

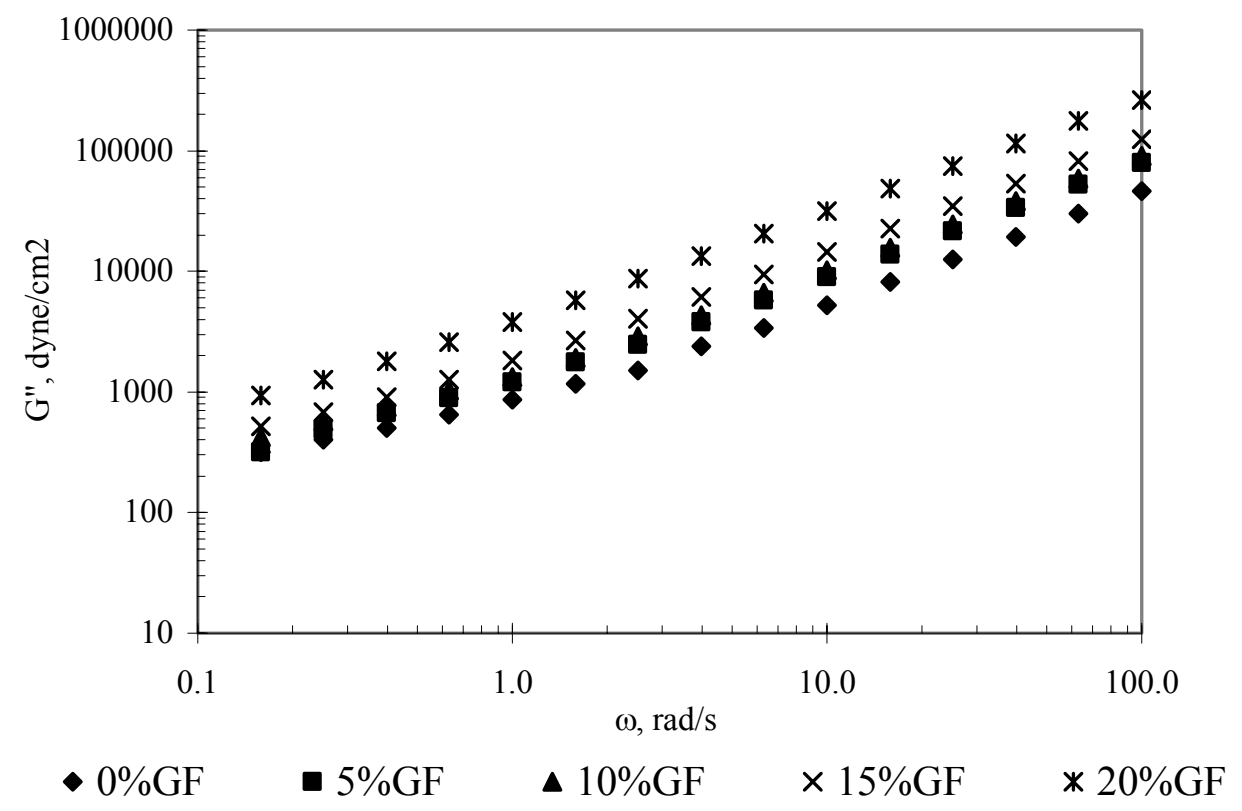

Figure 6-34 Recycled polycarbonate loss modulus vs. frequency $\left(\mathrm{T}=290{ }^{\circ} \mathrm{C}\right)$ 


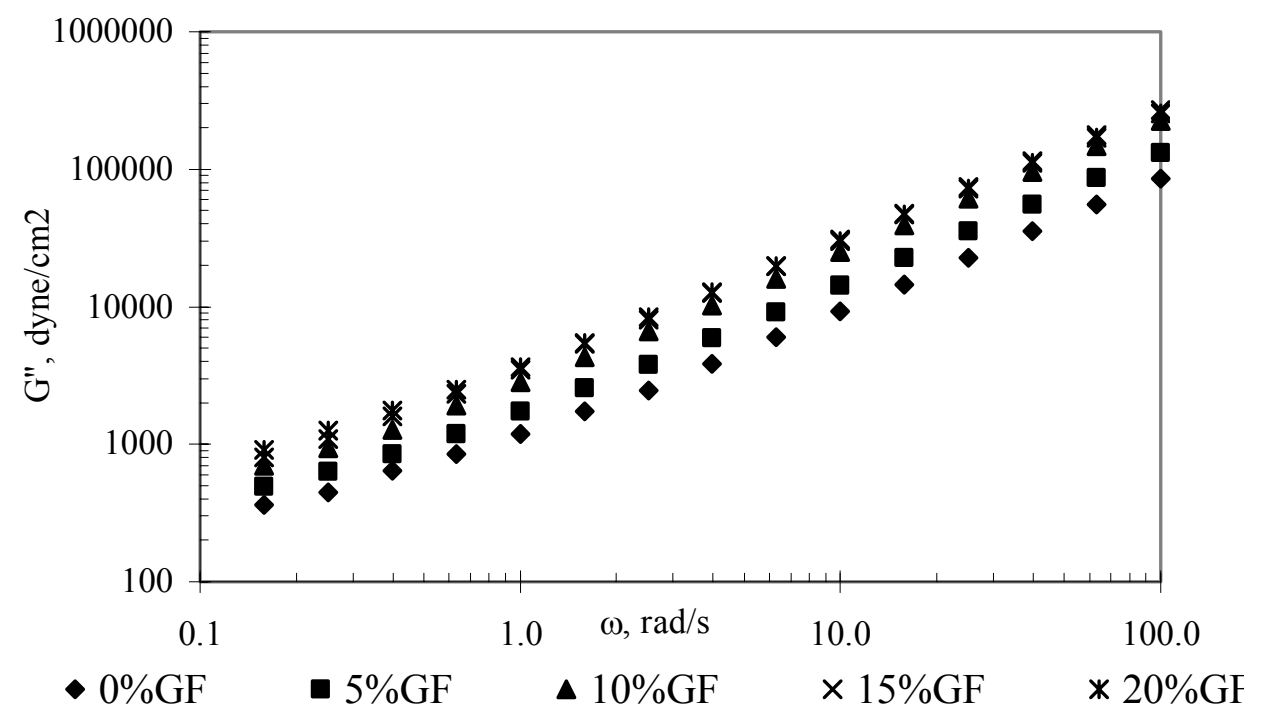

Figure $6-3550 \%$ virgin polycarbonate $+50 \%$ recycled polycarbonate loss modulus vs. frequency $\left(\mathrm{T}=290^{\circ} \mathrm{C}\right)$

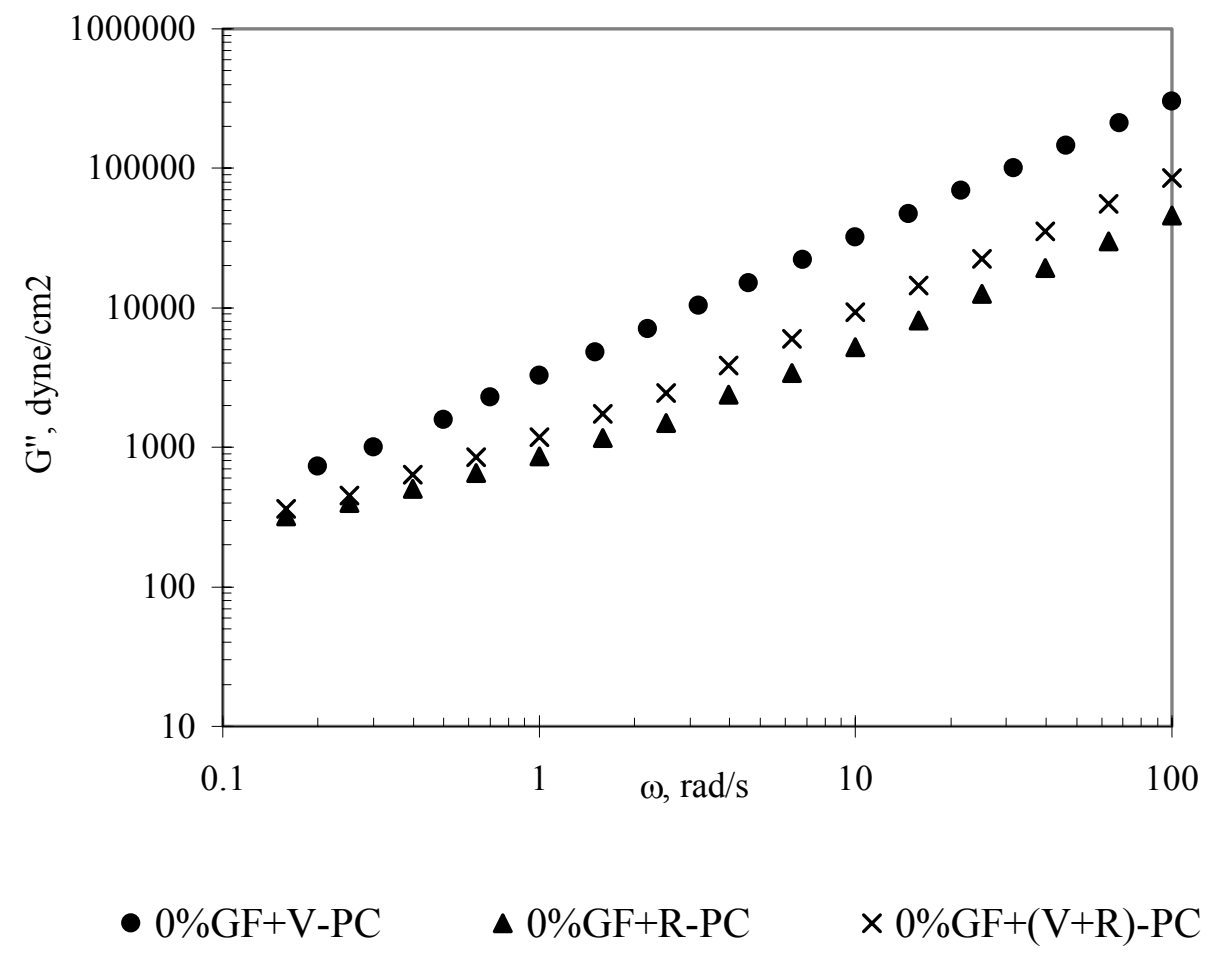

Figure 6-36 Unfilled polycarbonate loss modulus vs. frequency $\left(\mathrm{T}=290{ }^{\circ} \mathrm{C}\right)$ 


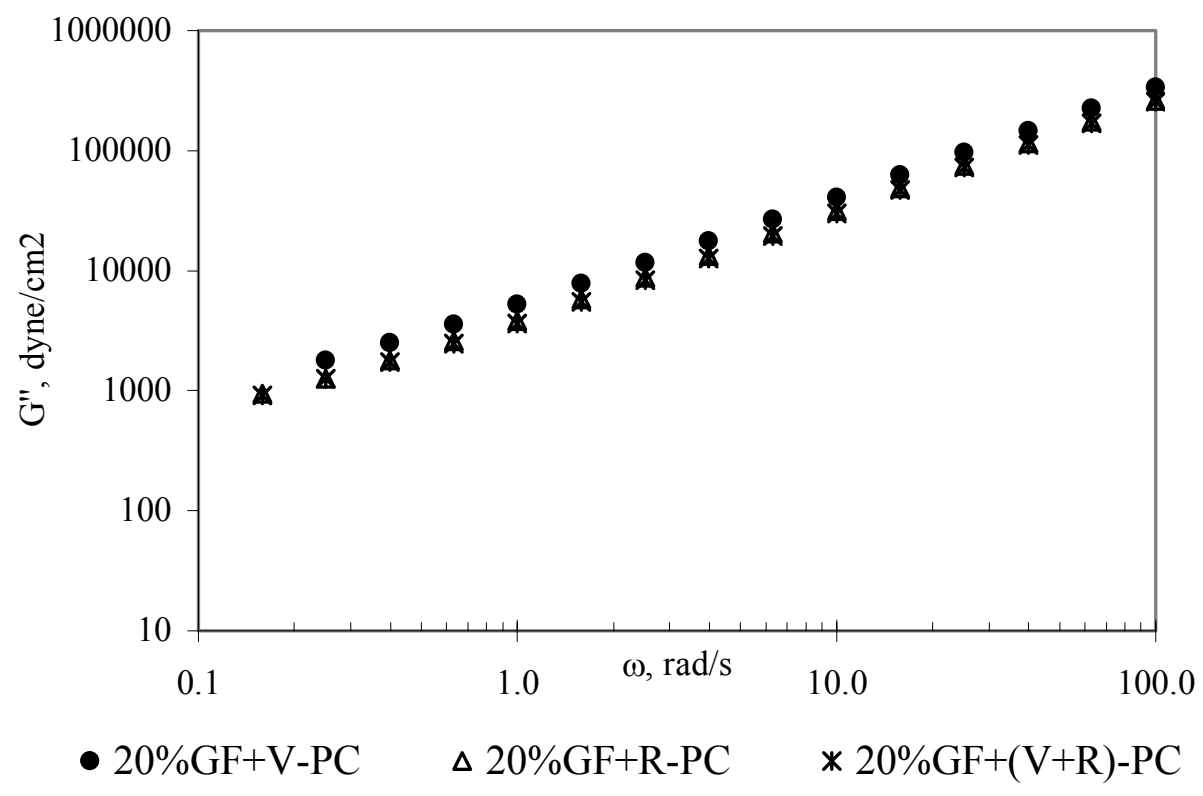

Figure 6-37 20\% glass fiber reinforced polycarbonate loss modulus vs. frequency ( $\mathrm{T}=290$ $\left.{ }^{\circ} \mathrm{C}\right)$

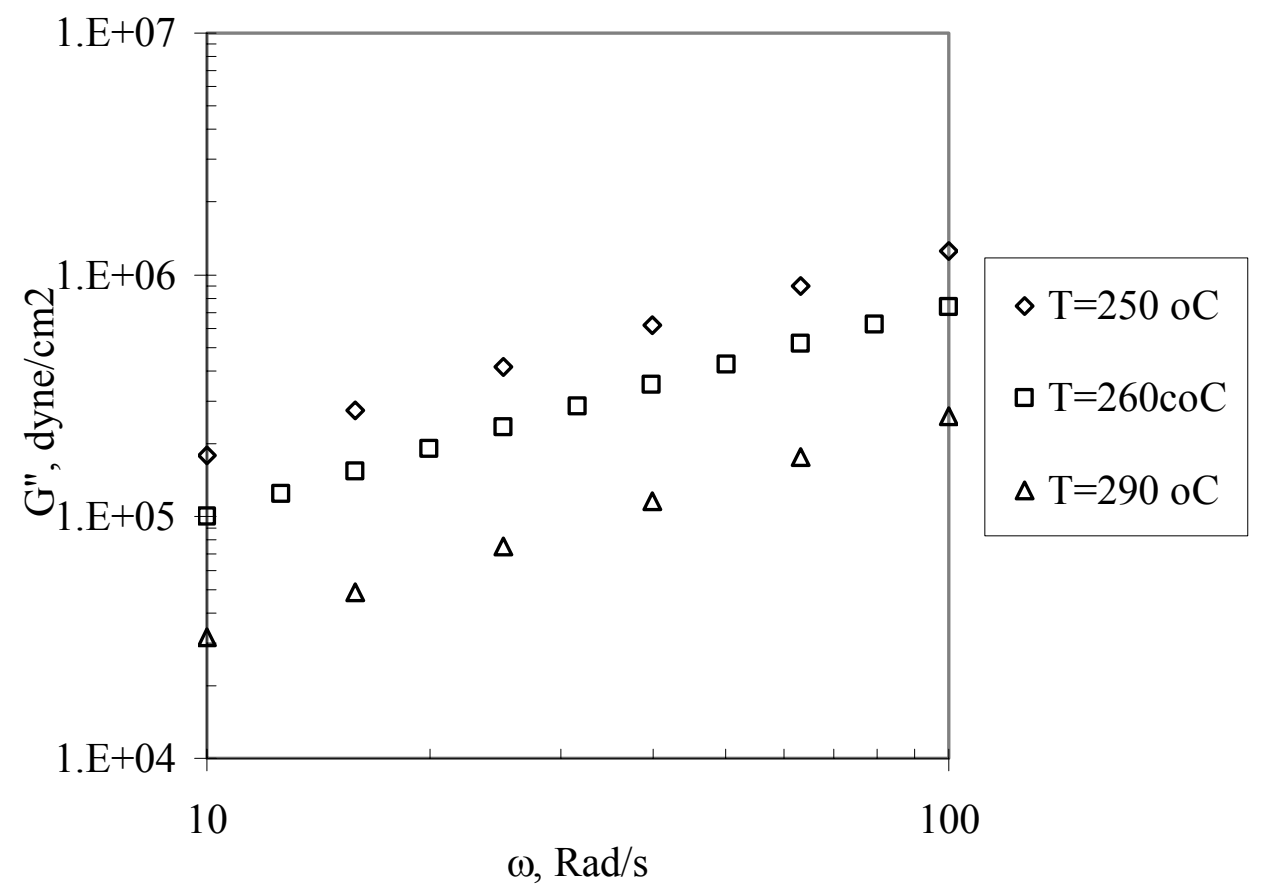

Figure 6-38 Influence of temperature on the loss modulus of $20 \%$ glass reinforced recycled $\mathrm{PC}$ 


\subsubsection{Prediction the storage and loss modulus}

The effect of frequency of oscillations, $\omega$, on the storage and loss moduli of polymer melts is conventionally described by the generalized Maxwell model ${ }^{67}$. The resulting functions of storage modulus (G') and storage modulus (G”) are;

$$
\begin{aligned}
& \mathrm{G}^{\prime}(\omega)=G \sum_{\mathrm{m}=1}^{\mathrm{M}} \mathrm{p}_{\mathrm{m}} \frac{\left(\omega \tau_{\mathrm{m}}\right)^{2}}{1+\left(\omega \tau_{\mathrm{m}}\right)^{2}} \\
& \mathrm{G}^{\prime \prime}(\omega)=\mathrm{G} \sum_{\mathrm{m}=1}^{\mathrm{M}} \mathrm{p}_{\mathrm{m}} \frac{\left(\omega \tau_{\mathrm{m}}\right)}{1+\left(\omega \tau_{\mathrm{m}}\right)^{2}}
\end{aligned}
$$

where $\mathrm{G}$ is the instantaneous shear modulus, $\mathrm{M}$ is the number of Maxwell elements (springs and dashpots connected in sequel see Figure 6-39) in the model, $\mathrm{p}_{\mathrm{m}}$ is the ratio of the elastic modulus of the $m$ th spring to the total elastic modulus, $\mathrm{G}$, and $\tau_{\mathrm{m}}$ is the relaxation time of the $m$ th dashpot. A shortcoming of Equations (6-9) and (6-10) is that for a limited set of frequencies, $\omega$, the problem of determining adjustable parameters in Equations (6-9) and (6-10) is ill-posed ${ }^{68}$ : small deviations of experimental data lead to large discrepancies in the constants $\left\{\mathrm{p}_{\mathrm{m}}, \tau_{\mathrm{m}}\right\}$. Several approaches have been proposed in the past decade for the regularization of this problem ${ }^{69,70}$. Although these methods allow the parameters, $\left\{\mathrm{p}_{\mathrm{m}}, \tau_{\mathrm{m}}\right\}$, to be found correctly for a given polymer melt, their applicability for the comparative study of the viscoelastic responses of different melts remains questionable.

A natural way to avoid this difficulty is to introduce additional assumptions regarding the shape of the relaxation spectrum, $\left\{\tau_{\mathrm{m}}\right\}$, based on a physically plausible scenario for the time-dependent response of a polymer melt at the micro-level. 
Two approaches are conventionally used for the scaling of relaxation times. According to the reptation concept ${ }^{71}$, relaxation of stresses in a polymer melt is associated with diffusion of segments of a representative chain in a tube to which the chain is confined by surrounding molecules. With reference to this model, the relaxation times, $\left\{\tau_{\mathrm{m}}\right\}$, are scaled as the characteristic times for diffusion. A disadvantage of this approach is that it reflects the time-dependent response of linear polymers, and cannot account for the presence of filler.

According to the theory of transient networks ${ }^{72,73,74}$, relaxation of stresses in a network of macromolecules is thought of as separation of active strands from their junctions (entanglements and physical cross-links) and merging of dangling strands with the network. The rearrangement events occur at random instants, as time strands are agitated by thermal fluctuations. This concept does not impose restrictions on the structure of macromolecules and can naturally take into account the presence of glass fibers (under the assumption that the filler particles are treated as extra junctions between chains). Within the framework on the theory of thermally activated processes ${ }^{75}$ this concept results in an exponential scaling of the relaxation times, $\tau_{\mathrm{m}}$, as functions of the activation energy for separation of strands from temporary junctions.

In the present study, we adopt the Green-Tobolsky theory ${ }^{76}$ for the analysis of the linear viscoelastic response of polymer melts, derive governing equations [similar to Equations (6-9) and (6-10)] for the storage and loss moduli that involve only 4 material constants, and find adjustable parameters in the stress-strain relations by fitting experimental data. 


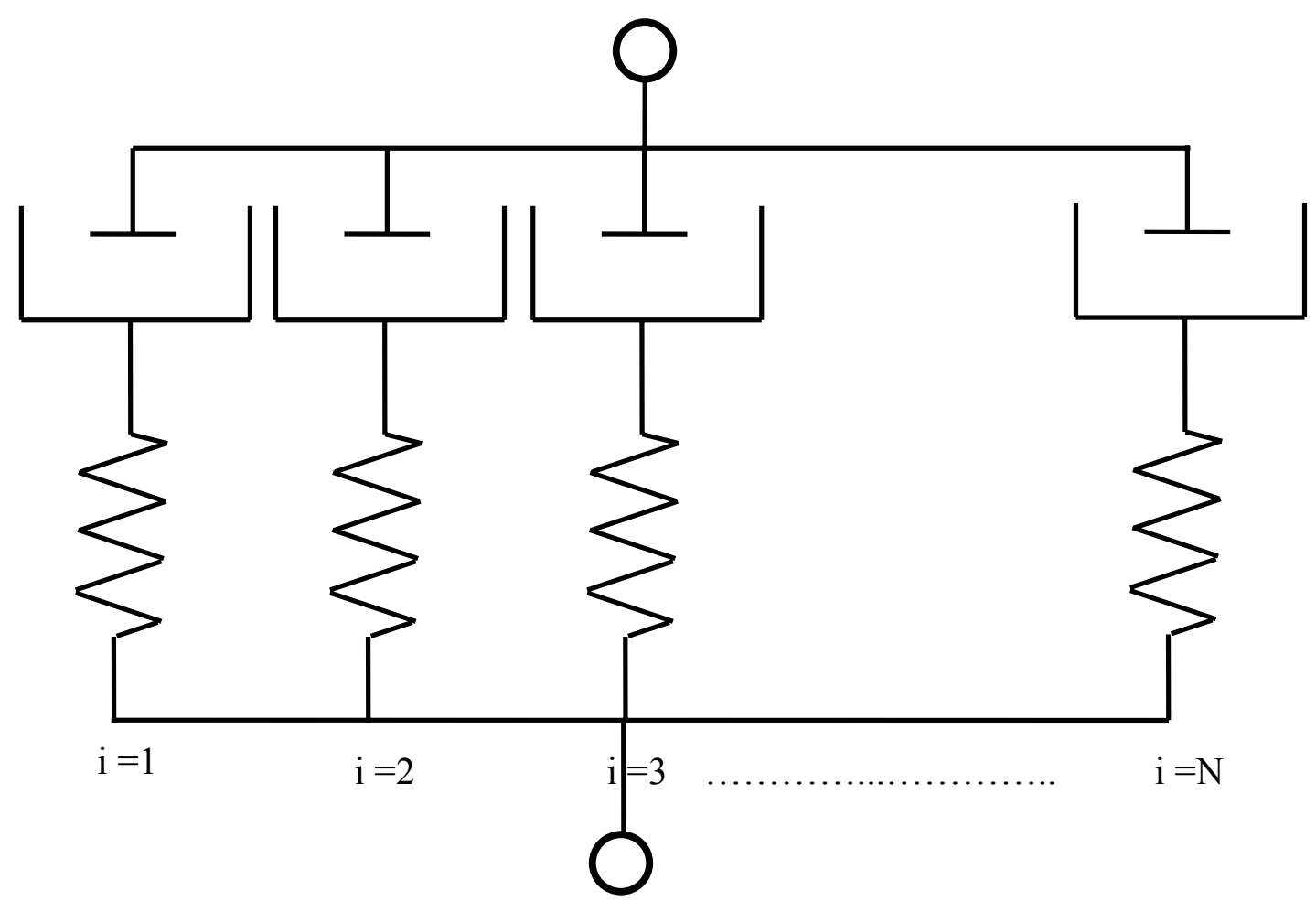

Figure 6-39 Mechanical analog of the generalized Maxwell model

A polymeric melt is modeled as an equivalent transient network of strands linked by temporary junctions (entanglements, physical cross-links and glass fibers). A strand whose ends are linked to contiguous junctions is treated as an active one. When an end of an active strand separates from a junction, the strand is transformed into the dangling strand. When a free end of a dangling strand captures a nearby junction, the strand returns into the active state.

Separation of active strands from their junctions and merging of dangling strands with the network are thought of as thermally activated processes which occur at random times when the strands are excited by thermal fluctuations. With reference to the theory of thermally activated processes, we assume that the rate of detachment, $\Gamma$, is governed 
by the Eyring equation

$$
\Gamma=\gamma \exp \left(\frac{-\overline{\mathrm{v}}}{\mathrm{k}_{\mathrm{B}} \mathrm{T}}\right)
$$

where $\gamma$ is the attempt rate, $\mathrm{k}_{\mathrm{B}}$ is Boltzmann's constant, $\mathrm{T}$ is the absolute temperature. and $\mathrm{v} \geq 0$ is the activation energy for separation of an active strand from a temporary junction. The pre-exponential factor $\gamma$ in Eq. (6-11) is independent of activation energy, $\overline{\mathrm{V}}$, and is determined by the current temperature $\mathrm{T}$ only.

Confining ourselves to isothermal processes at a reference temperature $T_{0}$ and introducing the dimensionless activation energy,

$$
\mathrm{v}=\frac{\overline{\mathrm{v}}}{\mathrm{k}_{\mathrm{B}} \mathrm{T}_{\mathrm{o}}}
$$

we find from Eq. (1) that

$$
\Gamma(\mathrm{v})=\gamma \exp (-\mathrm{v})
$$

To adequately describe the time-dependent response of a melt, we suppose that different junctions are characterized by different dimensionless activation energies $\mathrm{v}$. The distribution of active strands in a transient network is determined by the number of active strands per unit mass, $\mathrm{N}$, and the probability density $\mathrm{p}(\mathrm{v})$. The full derivation is given in Appendix E.

- Finally the parameter $\mathrm{p}_{\mathrm{m}}$ is replaced with $\mathrm{p}(\mathrm{v})$. Equations (6-9) and (6-10) become;

$$
\mathrm{G}^{\prime}(\omega)=\mathrm{G} \int_{0}^{\infty} \frac{\omega^{2}}{\gamma^{2} \exp (-2 \mathrm{v})+\omega^{2}} \mathrm{p}(\mathrm{v}) \mathrm{dv}
$$




$$
\mathrm{G}^{\prime \prime}(\omega)=\mathrm{G} \int_{0}^{\infty} \frac{\gamma \exp (-\mathrm{v}) \omega}{\gamma^{2} \exp (-2 \mathrm{v})+\omega^{2}} \mathrm{p}(\mathrm{v}) \mathrm{dv}
$$

The most important issue for the successful application of the governing equations to the approximation of the experimental data is the adequate choice of distribution function $\mathrm{p}(\mathrm{v})$. To match the observations in shear oscillatory tests we used the KWW (Kolhrausch-Williams-Watt) distribution expression for the function $\mathrm{p}(\mathrm{v})$. The KWW distribution function is given by;

$$
\mathrm{p}(\mathrm{v})=\mathrm{p}_{\mathrm{o}} \exp \left[-\left(\frac{\mathrm{v}}{\mathrm{V}}\right)^{\beta}\right] \quad(\mathrm{v} \geq 0), \quad \mathrm{p}(\mathrm{v})=0 \quad(\mathrm{v}<\mathrm{o})
$$

where $\beta$ and $\mathrm{V}$ are adjustable parameters and the coefficient $p_{o}$ is determined from normalization condition;

$$
\int_{0}^{\infty} p(v) d v=1
$$

The constitutive equations (6-13), (6-14) together with the phenomenological relation (615) involve 4 experimental constants:

1. the instantaneous shear modulus $\mathrm{G}$

2. the attempt rate for rearrangement of strands $\gamma$

3. the characteristic activation energy for rearrangement of strands V.

4. the dimensionless parameter $\beta$ that characterizes the stretched-exponential distribution function $\mathrm{p}(\mathrm{v})$.

The number of material parameters in the model is close to that employed in conventional phenomenological relations in linear viscoelasticity (such as the stretched exponential function used to fit data in creep and relaxation tests, or the standard 
viscoelastic solid). Application of more complicated relations (as a generalized Maxwell model with several relaxation times or rheological modes with fractional derivatives) requires a higher number of adjustable parameters to be found by matching observations, which makes the fitting algorithms unstable and frequently leads to physically unacceptable results (like negative coefficients in the Prony series).

Figure 6-40 and 6-41 show the fitted model using equations (6-13), (6-15), and (6-16) for unfilled virgin polycarbonate and $20 \%$ glass reinforced polycarbonate. Figures 6-40 and 6-41 demonstrate good agreement between the observations in oscillatory test and the results of numerical simulation. The rest of the curves with the adjustable parameters are given in Appendix E. 


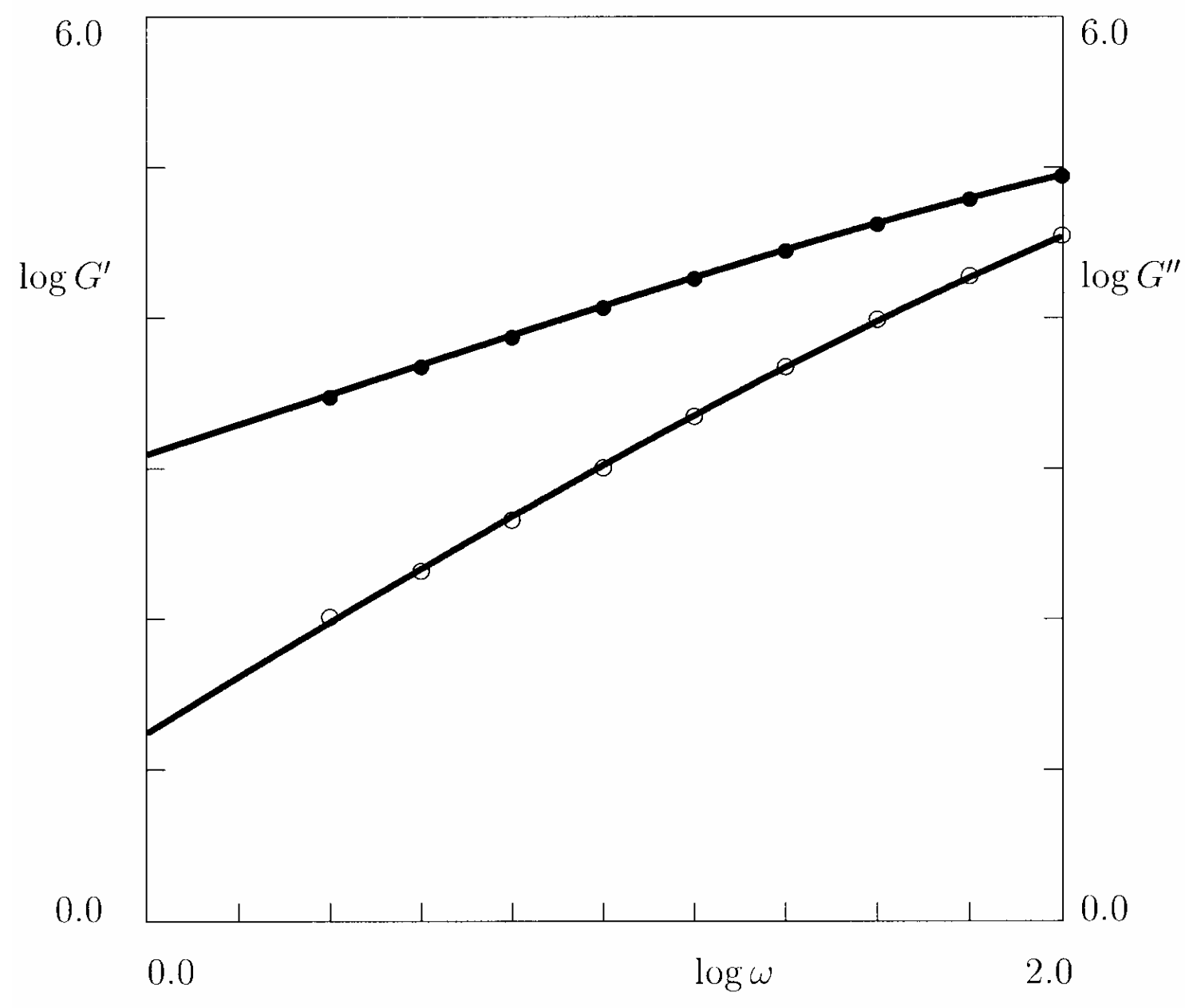

Figure 6-40 The storage modulus G' MPa (unfilled circles) and the loss modulus G" MPa (filled circles) versus frequency $\omega \mathrm{rad} / \mathrm{s}$. Symbols: experimental data for virgin PC at 250 ${ }^{\circ} \mathrm{C}$. Solid lines: results of numerical simulation 


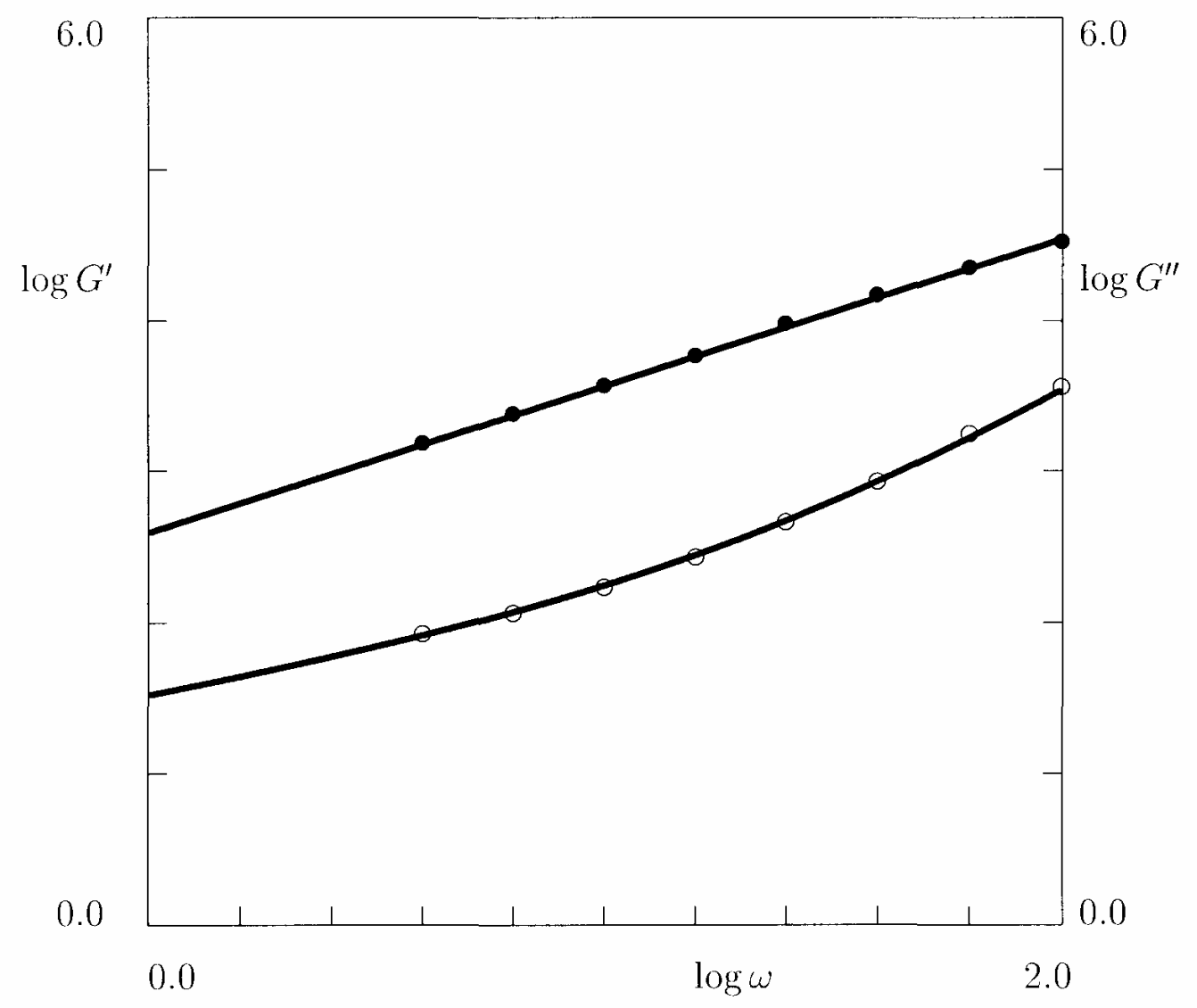

Figure 6-41 The storage modulus G' MPa (unfilled circles) and the loss modulus G' MPa (filled circles) versus frequency $\omega \mathrm{rad} / \mathrm{s}$. Symbols: experimental data for virgin PC with $20 \mathrm{wt} . \%$ of glass fibers at $290^{\circ} \mathrm{C}$. Solid lines: results of numerical simulation

\section{Discussion}

We begin with the study of the effects of temperature, $\mathrm{T}$, and filler fraction, $v$, on the adjustable parameters in the model for virgin PC. Figure 6-42 demonstrates that the instantaneous shear modulus, $\mathrm{G}$, of the unfilled melt of virgin $\mathrm{PC}$ at $290{ }^{\circ} \mathrm{C}$ exceeds that at $250{ }^{\circ} \mathrm{C}$. This conclusion is in accord with the conventional theory of rubber elasticity, according to which the modulus of a polymeric melt increases with temperature $\mathrm{T}$ (due to the growth of entropy of chains). It should be noted, however, that the increase of G with 
temperature (by about 90\%) noticeably exceeds that predicted by the concept of rubbery elasticity (which postulates that $\mathrm{G}$ is proportional to $\mathrm{T}$ ). The latter means that the concentration of temporary junctions in an equivalent network increases with $\mathrm{T}$.

Curve 1 in Figure 6-42 shows that at $250{ }^{\circ} \mathrm{C}$, the instantaneous shear modulus, G, grows with the content of glass fibers, $v$. The modulus, G, increases by approximately $40 \%$ (when $v$ changes from 0 to 20 wt.-\%), which implies that at this temperature, the glass fibers are linked with the polymeric matrix rather strongly.

According to curve 2 in Figure 6-42, the situation changes dramatically when the temperature is increased up to $290^{\circ} \mathrm{C}$. At this temperature, the shear modulus, G, noticeably decreases with $v$ (by $61 \%$ when $v$ grows from 0 to 15 wt.- $\%$ ). To explain this observation, we assume that inter actions between the polymeric matrix and glass fibers pronouncedly weaken with temperature. Due to this decay in the strength of interactions, the macro-strain is not transformed to all glass fibers by the host matrix, which implies that the modulus does not grow with $v$ The decrease in $\mathrm{G}(v)$ is attributed to aggregation of glass fibers into clusters and formation of occluded domains (similar to occluded regions in rubber reinforced with filler particles), where the macro-strain is screened by surrounding aggregates of short fibers. When the concentration of filler exceeds the percolation threshold (the latter is located in the interval between 10 and $20 \mathrm{wt.}-\%$, in accord with conventional percolation theories), the shear modulus begins to grow. This increase in $\mathrm{G}$ (observed as a deviation of the experimental point corresponding to $v=20$ wt.- $\%$ from the prediction given by curve 2 in Figure 6-42) is associated with formation of a secondary network of glass fibers that carries out a part of external load. 


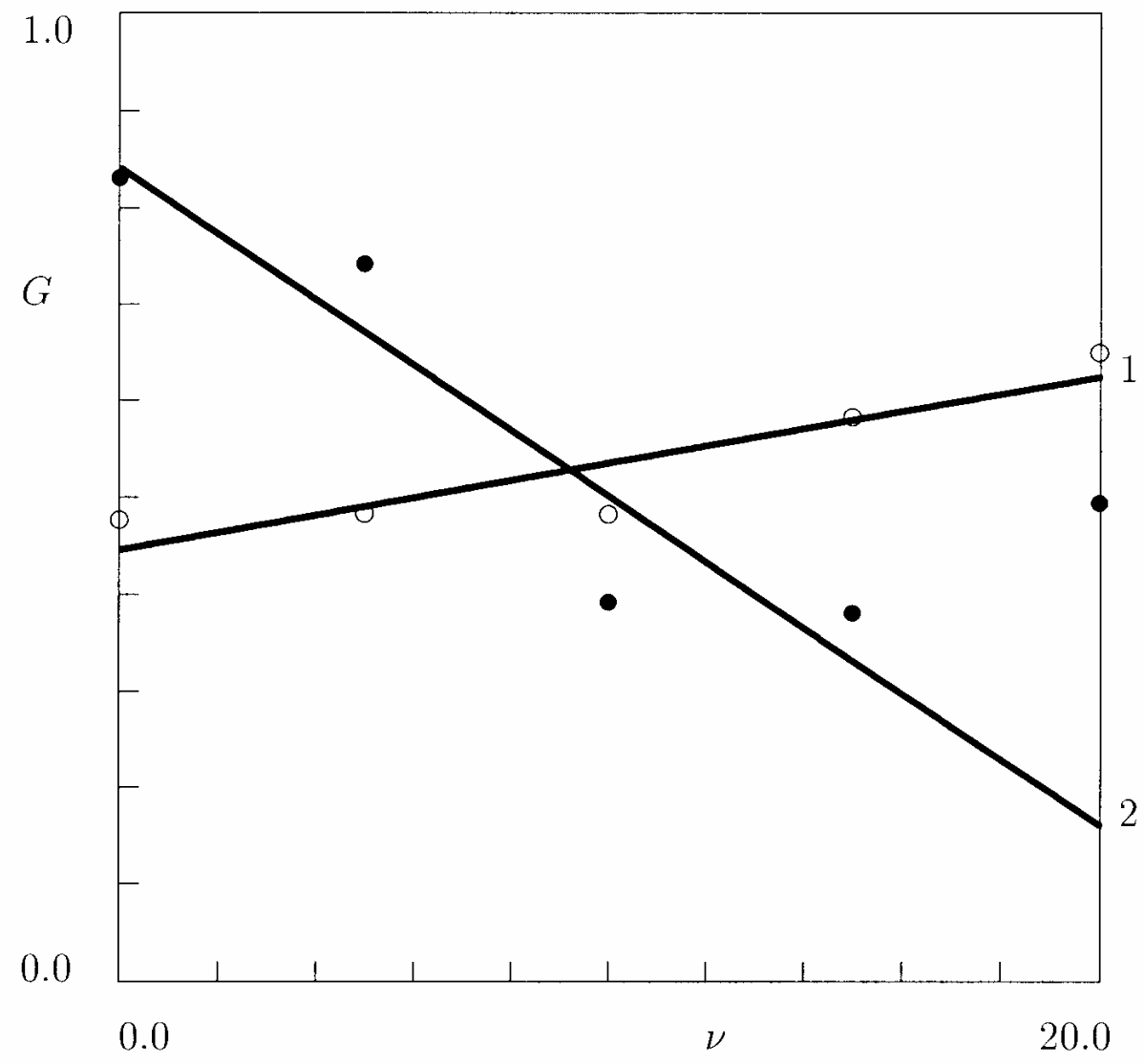

Figure 6-42 The shear modulus G' MPa versus the filler content $v$ wt.\%. Symbols: treatment of observations for virgin $\mathrm{PC}$ at $250{ }^{\circ} \mathrm{C}$ (unfilled circles) and $290{ }^{\circ} \mathrm{C}$ (filled circles). Solid lines: approximation of the experimental data by the function $\mathrm{G}=\mathrm{G}_{0}+\mathrm{G}_{1} v$. Curve 1: $\mathrm{G}_{\mathrm{o}}=0.45, \mathrm{G}_{1}=8.92$ E10-3 curve $2: \mathrm{G}_{\mathrm{o}}=0.84, \mathrm{G} 1=-3.40 \mathrm{E}-2$

Figure 6-43 demonstrates that the dimensionless parameter $Z$ (that characterizes the average energy for rearrangement of strands) weakly decreases with the content of glass fibers, $v$ at $250{ }^{\circ} \mathrm{C}$ and the rate of decrease noticeably grows when the temperature reaches $290{ }^{\circ} \mathrm{C}$. According to Figure 6-43, the average energy of thermal fluctuations necessary for separation of an active strand from a temporary junction diminishes with the growth of the filler content. This observation seems quite natural if we recall that both 
entanglements between chains and glass fibers are treated as temporary junctions in an equivalent network. When the content of filler increases, the number of strands (per unit mass) connected to glass fibers grows as well. As interactions between polymeric chains and glass fibers are rather weak (their strength is substantially less than the strength of entanglements between chains), the average energy for detachment of strands from temporary junctions decreases noticeably.

The fact that interactions between polymeric chains induced by their entanglements are stronger than those between chains and glass fibers provides also an explanation for the difference between curves 1 and 2 in Figure 6-43. As it was mentioned above, interactions between the host matrix and glass fibers weaken dramatically with temperature. This implies that the higher the temperature, $\mathrm{T}$, is, the smaller is the energy necessary for separation of a polymeric chain from a glass fiber. According to this assertion, a decrease in the average activation energy for detachment of strands with $v$ should be more pronounced at a higher temperature. This conclusion is fairly well confirmed by the experimental data depicted in Figure 6-43, where curve 1 is located noticeably higher than curve 2 . 


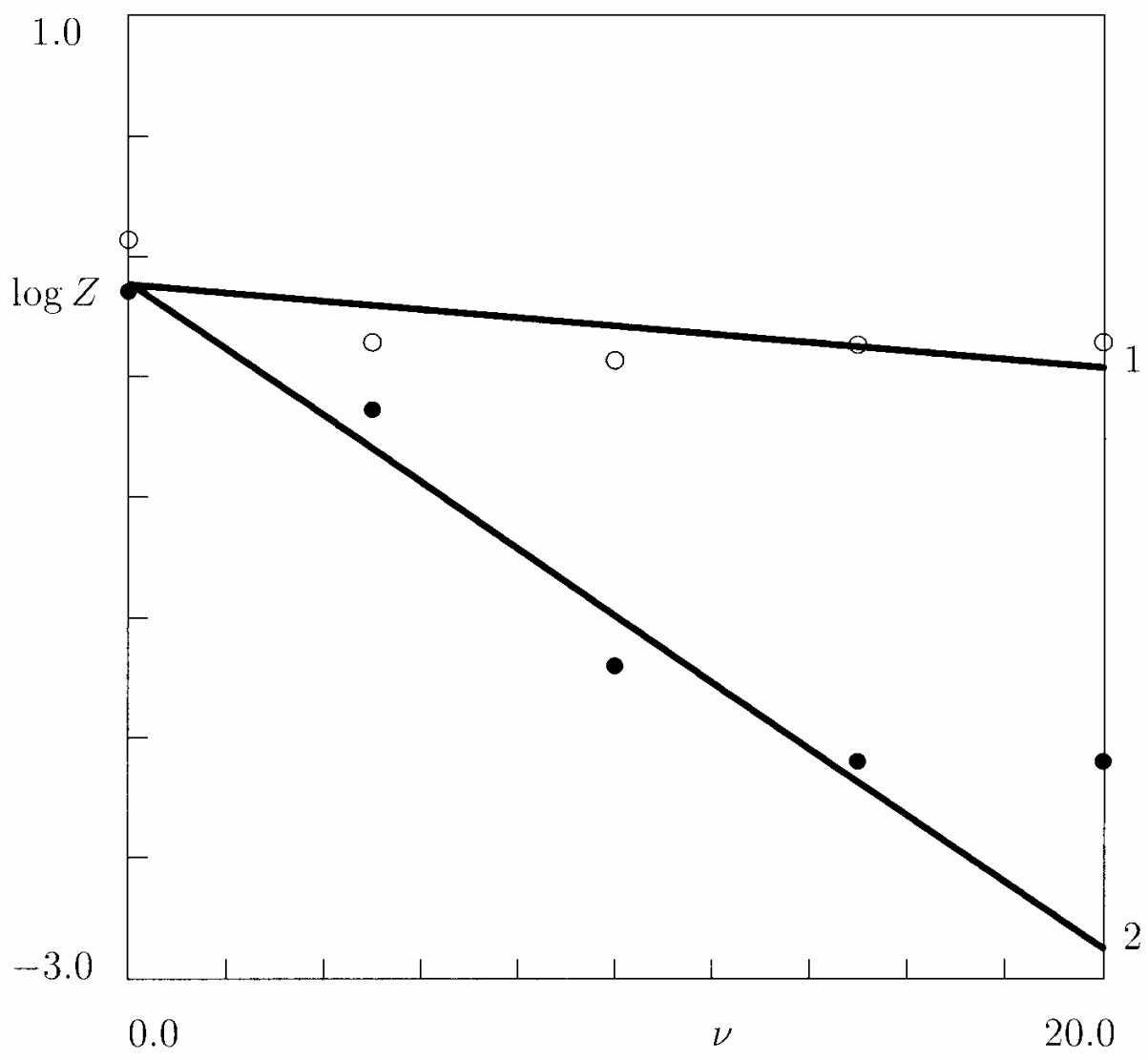

Figure 6-43: The average activation energy $Z$ versus the filler content $v$ wt.- $\%$. Symbols: treatment of observations for virgin $\mathrm{PC}$ at $250{ }^{\circ} \mathrm{C}$ (unfilled circles) and $290{ }^{\circ} \mathrm{C}$ (filled circles). Solid lines: approximation of the experimental data by the function $\log Z=Z_{o}-$ $Z_{1} v$. Curve $1: Z_{\mathrm{o}}=1.15 \mathrm{E}-1, Z_{1}=1.73 \mathrm{E}-2$; curve $2: Z_{\mathrm{o}}=-1.06 \mathrm{E}-1, Z_{1}=1.38 \mathrm{E}-1$

Figure 6-44 shows at all concentrations of glass fibers, the attempt rate, $\gamma$, at 290 ${ }^{\circ} \mathrm{C}$ exceeds that at $250{ }^{\circ} \mathrm{C}$. This observations is in agreement with our treatment of separation of active strands from temporary junctions as a thermally-activated process. The latter means that the higher the temperature is, the larger is the rate of rearrangement.

According to Figure 6-44, the attempt rate, $\gamma$, weakly decreases with the filler content, $v$, at $250{ }^{\circ} \mathrm{C}$, and strongly falls down with $v$ at $290{ }^{\circ} \mathrm{C}$. These observations appear 
to be quite natural, because a decrease in $\gamma(v)$ is tantamount to a decrease in mobility of polymeric chains. The latter is associated with restrictions on thermal movement of macromolecules near the surfaces of filler particles (where fibers serve as obstacles for the motion of chains). At the low temperature, $\mathrm{T}=250^{\circ} \mathrm{C}$, these limitations are not rather strong, because thermal motion of chains is relatively slow and the time necessary for a strand located in a close vicinity of a glass fiber to investigate all available configurations is comparable to that for a strand in the neat polymer (the latter implies that the decrease in $\gamma(v)$ is not rather pronounced, as it is revealed by curve 1 in Figure 6-44). With the growth of temperature to $\mathrm{T}=290^{\circ} \mathrm{C}$, the effect of restrictions on available configurations of a strand increases dramatically (due to an increase in the rate of thermal motion), which results in a noticeable decrease in the attempt rate $\gamma$ (see curve 2 in Figure 6-44). 


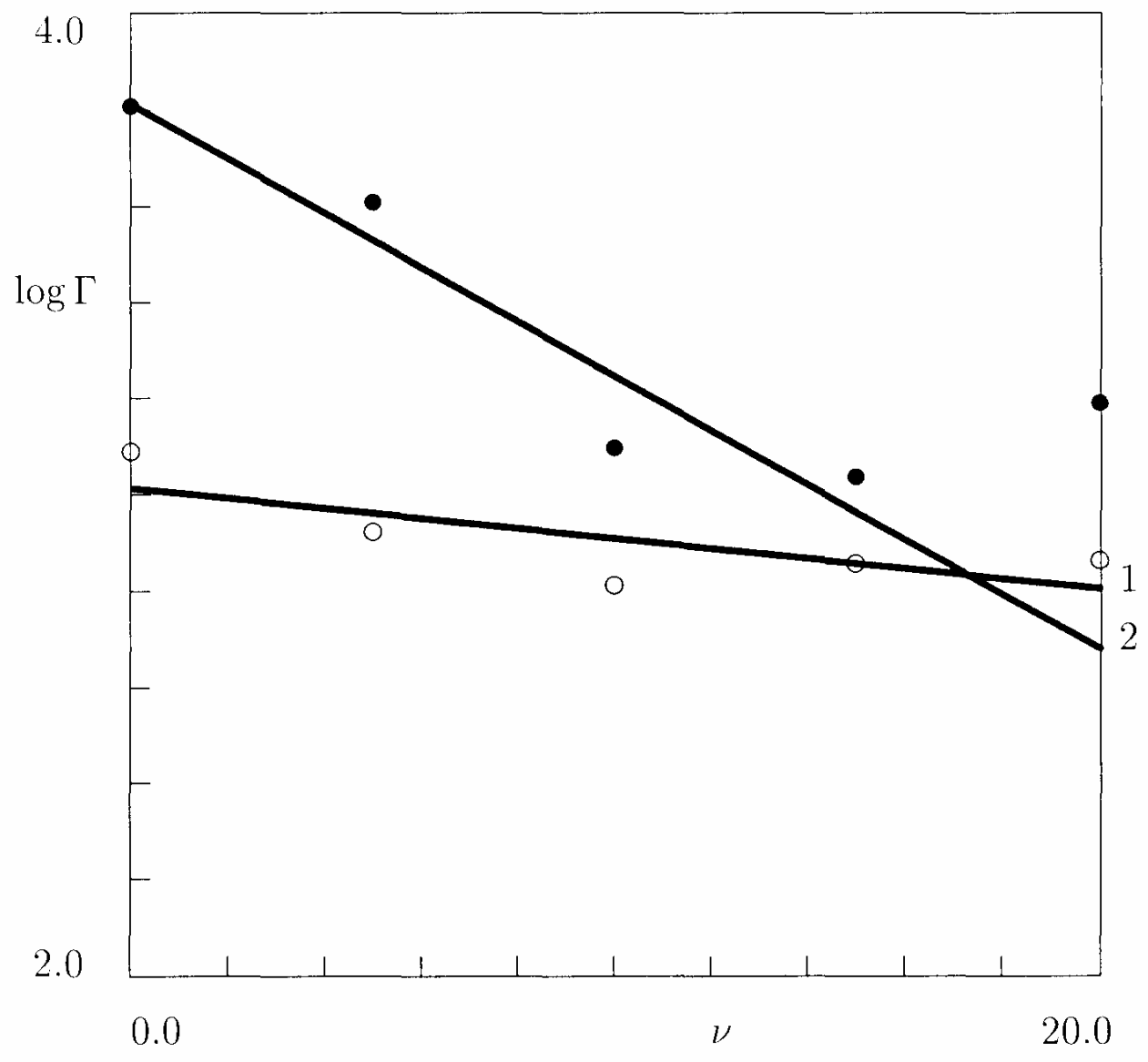

Figure 6-44: The attempt rate $\Gamma_{\mathrm{o}} \mathrm{s}^{-1}$ versus the filler content $v$ wt.-\%. Symbols: treatment of observations for virgin $\mathrm{PC}$ at $250{ }^{\circ} \mathrm{C}$ (unfilled circles) and $290{ }^{\circ} \mathrm{C}$ (filled circles). Solid lines: approximation of the experimental data by the function $\log \Gamma_{0}=\gamma_{0}-\gamma_{1} v$. Curve 1: $\gamma_{0}=3.01, \gamma_{1}=1.04 \mathrm{E}-2$; curve $2: \gamma_{0}=3.81, \gamma_{1}=5.66 \mathrm{E}-2$

Figure 6-45 demonstrates that the parameter $\beta$ (that characterizes the width of the distribution function $\mathrm{p}$ plotted versus $\mathrm{v} / \mathrm{V}$ ) decreases with $v$ at both temperatures. This observation together with Eq. (6-15) implies that the width of the distribution function grows with the content of filler. This conclusion also seems rather natural. It means that with an increase in the content of glass fibers, interactions between chains in the host matrix and fibers become less homogeneous. An increase in the heterogeneity of 
interactions is reflected by strengthening of a "tail" of the distribution function $\mathrm{p}$.

According to Figure 6-45, at all concentrations of filler, $v$, the values of $\beta$ at 250 ${ }^{\circ} \mathrm{C}$ exceed those at $290{ }^{\circ} \mathrm{C}$. This means that the growth of temperature results in an increase in the inhomogeneity of interactions between polymeric chains and glass fibers. The rate of decrease in $\beta$ with $v$ at $290{ }^{\circ} \mathrm{C}$ slightly exceeds that at $250{ }^{\circ} \mathrm{C}$, but the scatter of experimental data is rather large to make a definite conclusion.

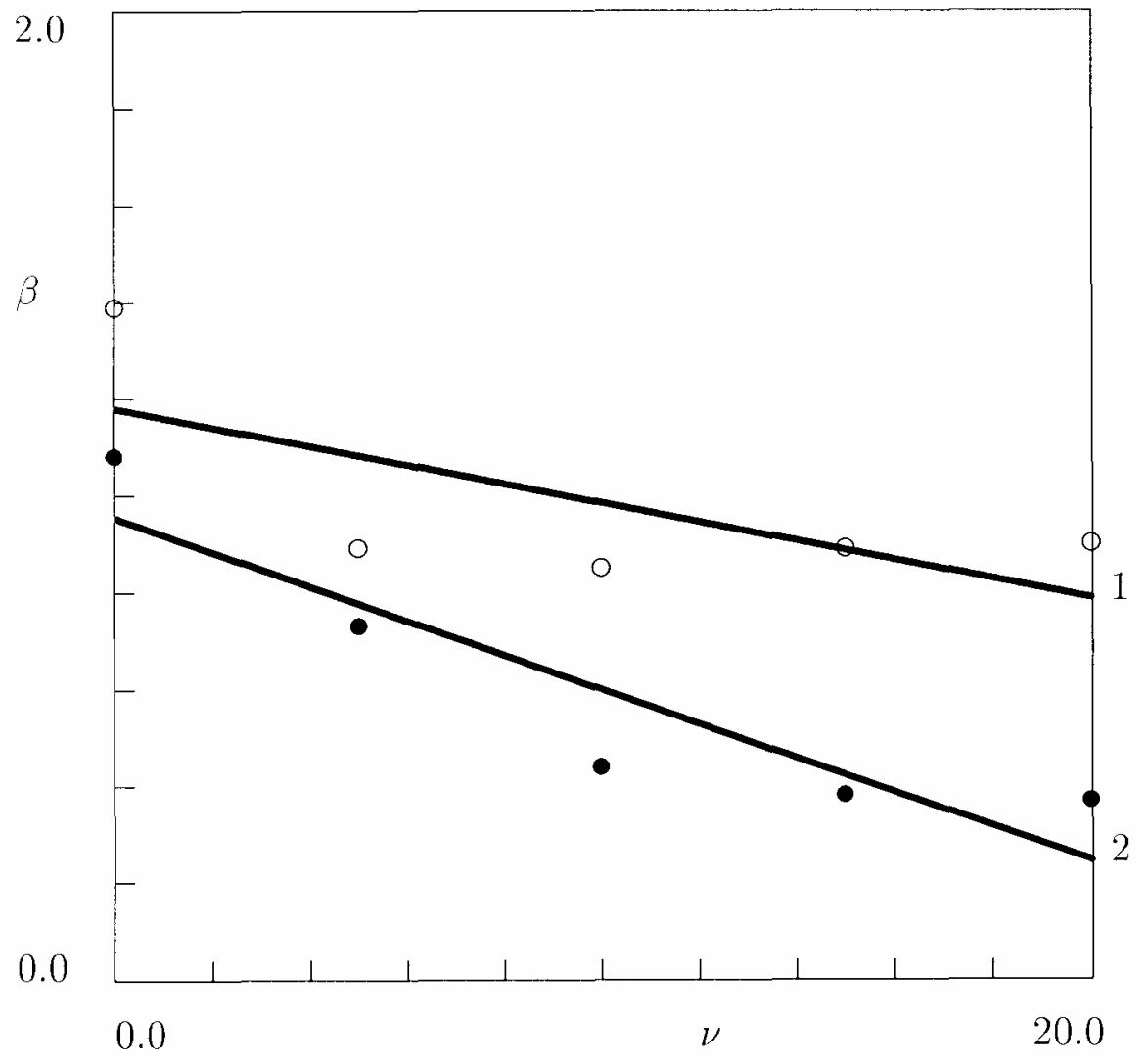

Figure 6-45: The dimensionless parameter $\beta$ versus the filler content $v$ wt.-\%. Symbols: treatment of observations for virgin $\mathrm{PC}$ at $250{ }^{\circ} \mathrm{C}$ (unfilled circles) and $290{ }^{\circ} \mathrm{C}$ (filled circles). Solid lines: approximation of the experimental data by the function $\beta=\beta_{0}-\beta_{1} v$. Curve 1: $\beta_{\mathrm{o}}=1.18, \beta_{1}=1.96 \mathrm{E}-2$; Curve $2: \beta_{\mathrm{o}}=0.95, \beta_{1}=3.54 \mathrm{E}-2$. 
Our aim now is to compare results of numerical simulation for the melts of virgin $\mathrm{PC}$, recycled $\mathrm{PC}$ and a mixture of virgin and recycled $\mathrm{PC}$ at a fixed temperature, $\mathrm{T}=250$ ${ }^{\circ} \mathrm{C}$.

Figure 6-46 shows that the shear modulus, G, slightly increases with $v$ for virgin PC, and decreases for recycled PC and a mixture of virgin and recycled polymers. With reference to the above analysis for the effect of temperature on the elastic modulus for virgin $\mathrm{PC}$, we conclude that the decrease in the function $\mathrm{G}(v)$ reflects (i) rather weak interactions between polymeric chains and glass fibers in the recycled polymer and (ii) a higher degree of aggregation of fibers into clusters in recycled PC compared to the virgin polymer.

Figure 6-46 reveals that at all concentrations of glass fibers, $v>0$, the elastic modulus of virgin PC exceeds that of recycled PC, whereas the modulus of the mixture of the two polymers is located in between the two extremes. This observation may be ascribed to weakening of interactions between chains and fibers in the recycled material compared to virgin polycarbonate. 


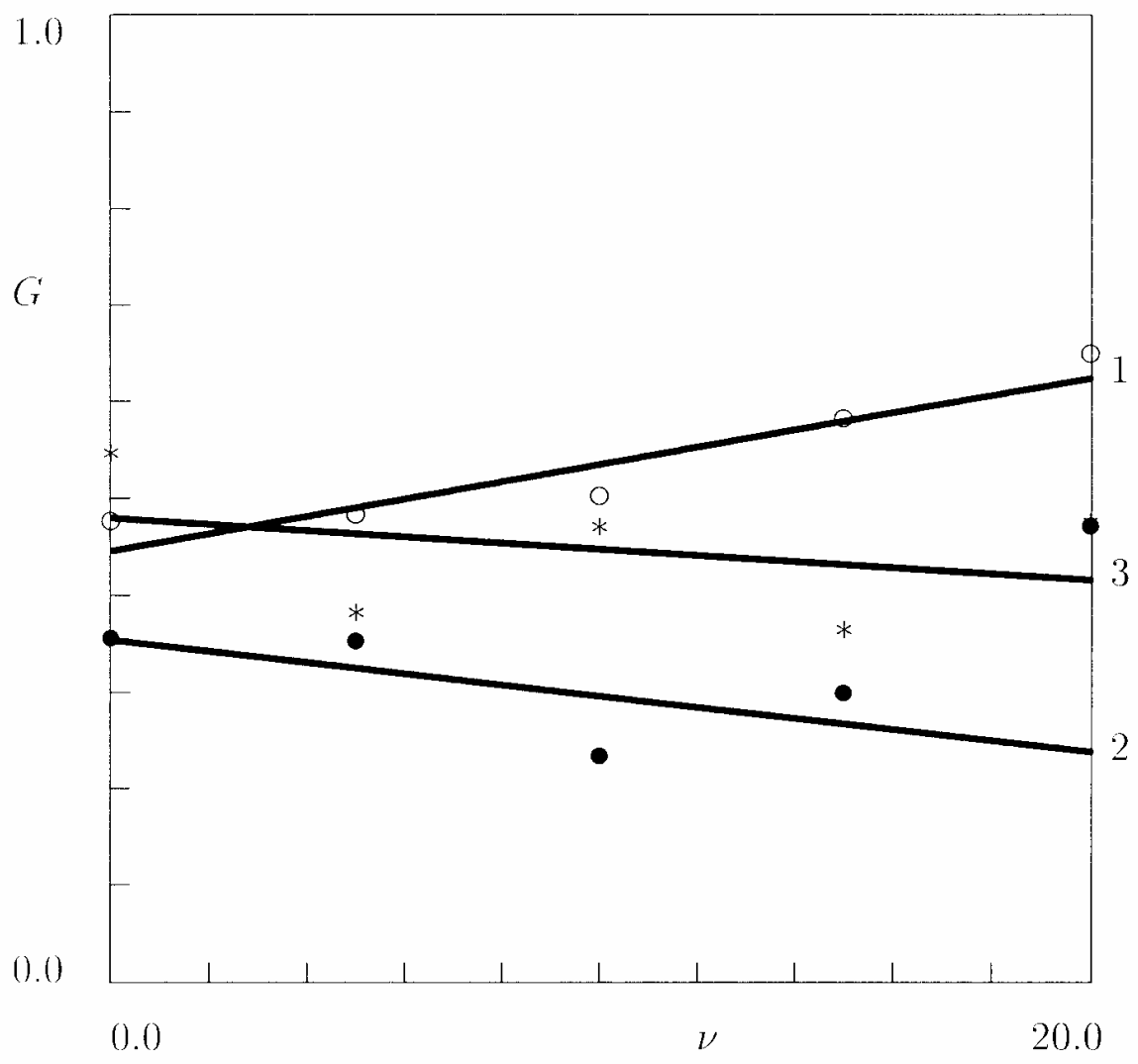

Figure 6-46 The shear modulus $\mathrm{G} \mathrm{MPa}$ versus the filler content $v \mathrm{wt} \%$. Symbols: treatment observation at $250{ }^{\circ} \mathrm{C}$ on virgin PC (unfilled circles), recycled PC (filled circles) and the mixture of $50 \mathrm{wt} \%$ virgin $\mathrm{PC}$ with $50 \mathrm{wt} \%$ recycled $\mathrm{PC}$ (asterisks). Solid lines: approximation of the experimental data by function $\mathrm{G}=\mathrm{G}_{\mathrm{o}}-\mathrm{G}_{1}$ v. Curve 1: $\mathrm{G}_{\mathrm{o}}=$ $0.45, \mathrm{G}_{1}=-8.92 \mathrm{E}-3$; curve $2: \mathrm{G}_{\mathrm{o}}=0.35, \mathrm{G}_{1}=5.8 \mathrm{E}-3$; curve $3: \mathrm{G}_{\mathrm{o}}=0.48, \mathrm{G}_{1}=3.2 \mathrm{E}-3$

According to Figure 6-47, the average activation energy, V, for separation of strands from temporary junctions decreases with the content of glass fibers for al three materials. The decrease is less pronounced for virgin PC, and it becomes substantially stronger for recycled PC. The decrease in $\mathrm{V}$ for the mixture of virgin and recycled PC is intermediate between those for virgin and recycled materials (which means that an analog 
of the rule of mixture may be employed to assess the average activation energy for the mixture). Comparison of Figures 4-43 and 6-47 implies that the average energy for rearrangement of strands in a transient network corresponding to recycled PC is substantially less than that for virgin polycarbonate, which may be explained by pronounced weakening of interactions between polymeric chains and fibers.

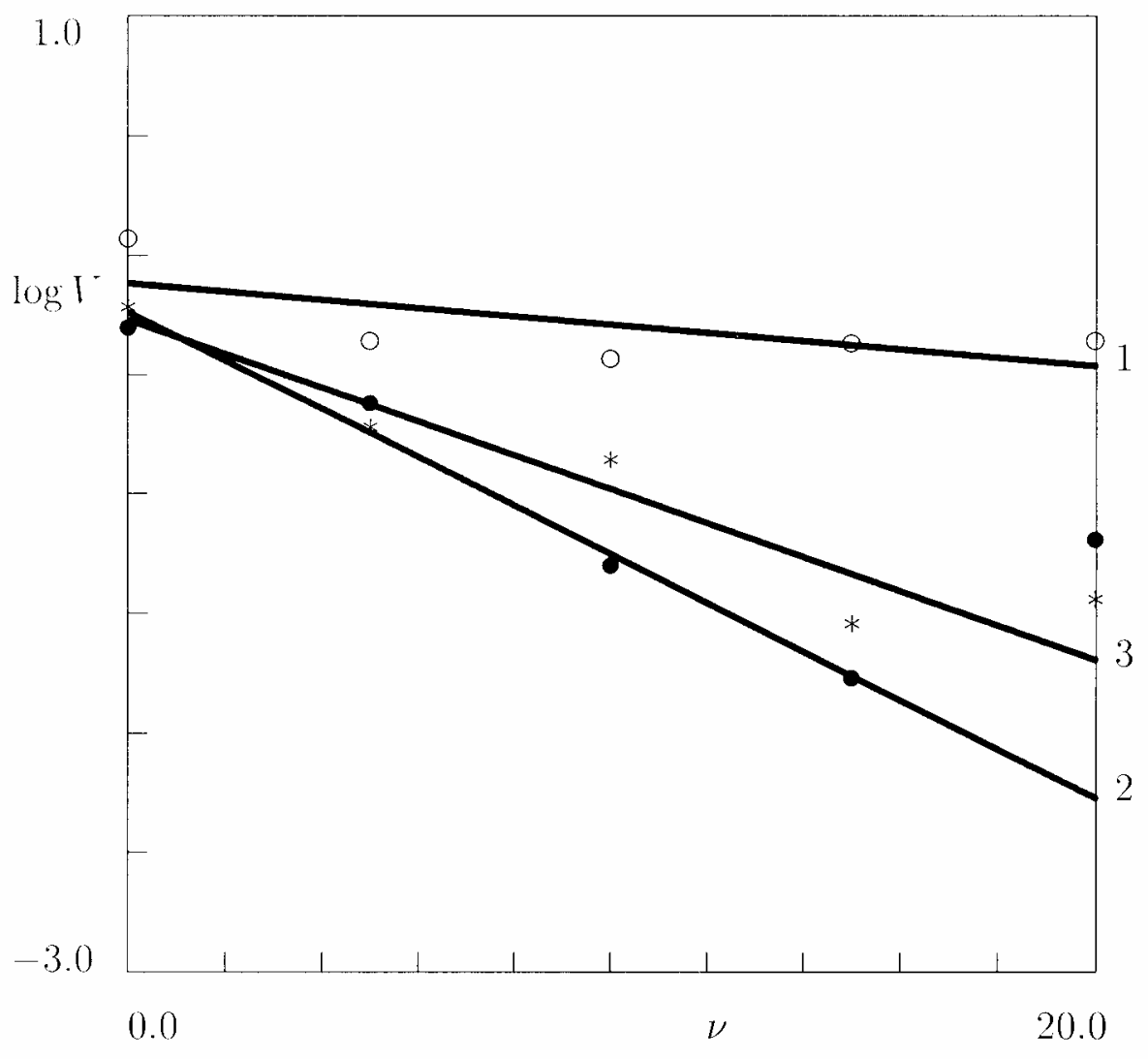

Figure 6-47: The dimensionless parameter $\mathrm{V}$ versus the filler content $v \mathrm{wt} \%$. Symbols: treatment observation at $250{ }^{\circ} \mathrm{C}$ on virgin PC (unfilled circles), recycled PC (filled circles) and the mixture of $50 \mathrm{wt} \%$ virgin PC with $50 \mathrm{wt} \%$ recycled PC (asterisks). Solid lines: approximation of the experimental data by function $\log \mathrm{V}=\mathrm{V}_{\mathrm{o}}-\mathrm{V}_{1} v$. Curve $1: \mathrm{V}_{\mathrm{o}}=$ $-0.11, \mathrm{~V}_{1}=1.73 \mathrm{E}-2$; curve $2: \mathrm{V}_{\mathrm{o}}=-0.23, \mathrm{~V}_{1}=1.02 \mathrm{E}-1$; curve $3: \mathrm{V}_{\mathrm{o}}=-0.27, \mathrm{G}_{1}=7.12 \mathrm{E}-2$ 
Figure 6-48 shows that the attempt rate, $\gamma$, decreases with $v$ for all three melts. The rate of decay is the highest for recycled PC, the smallest for virgin PC, and intermediate for the mixture of the two polymers. For all concentrations of filler, $v>0$, the attempt rate, $\gamma$, for virgin PC exceeds that for recycled PC and the mixture of virgin and recycled materials (due to the large scatter of experimental data it is difficult to distinguish between the values of $\gamma$ for recycled polycarbonate and the mixture of virgin and recycled polymers). The fact that the attempt rate for virgin polycarbonate is higher that that for recycled PC may be associated with chemical degradation of the recycled material. Conventional models for degradation of polymers presume that diffusion of oxygen results in scission of long chains in the surface layers of polymeric articles. This means that (independently of the initial molecular weight of a polymer), the number of short chains in the recycled material exceeds that in the virgin one. As short chains act as extra physical cross-links between polymeric chains, an increase in their concentration implies a decrease in segmental mobility of macromolecules, which is reflected in Figure 17 as a decrease in $\gamma$ for recycled PC compared to the virgin polymer. 


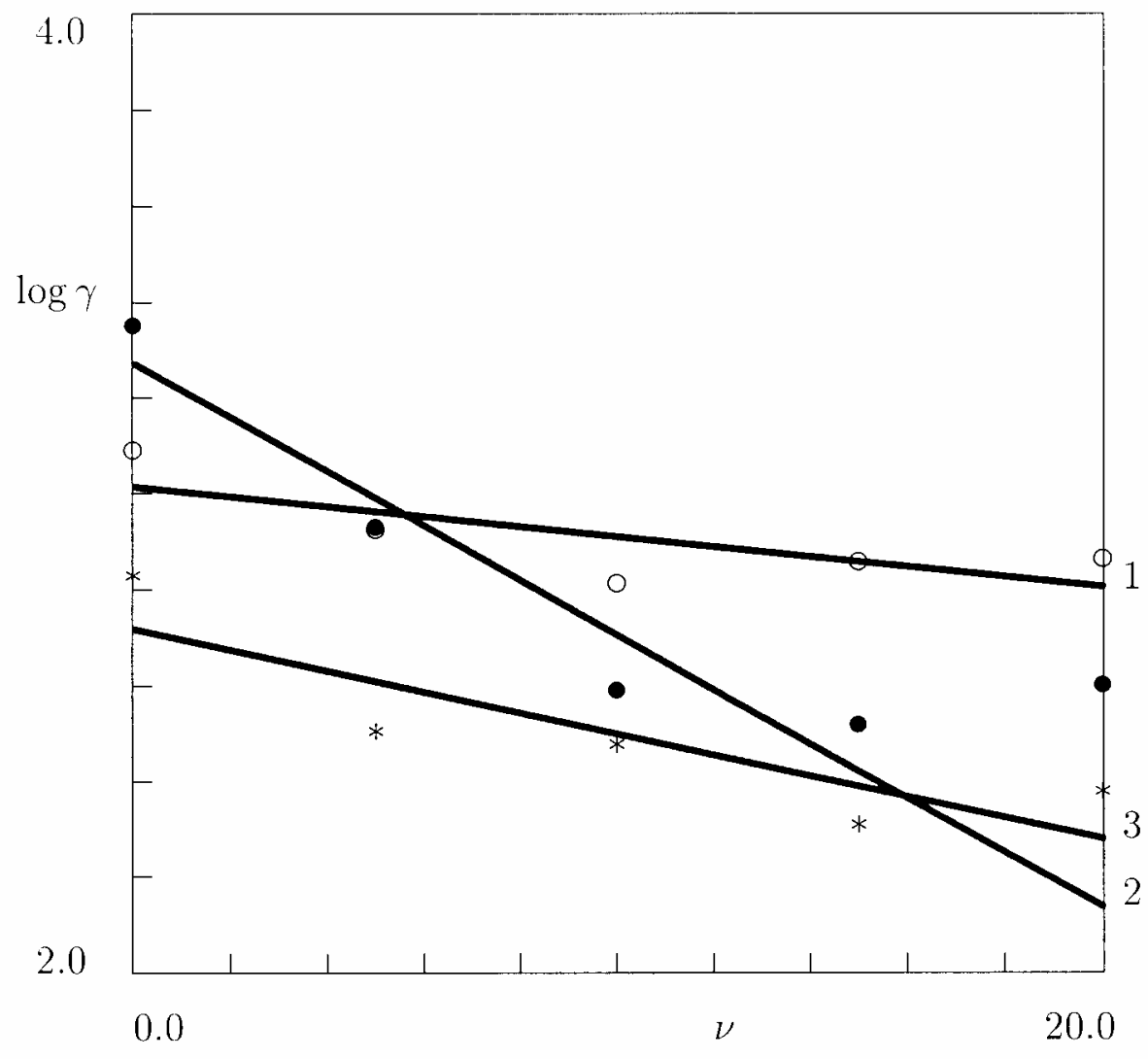

Figure 6-48: The attempt rate $\gamma \mathrm{s}^{-1}$ versus the filler content $v$ wt.- $\%$. Symbols: treatment of observations at $250{ }^{\circ} \mathrm{C}$ on virgin PC (unfilled circles), recycled PC (filled circles) and the mixture of 50 wt.- $\%$ virgin with 50 wt.- $\%$ recycled PC (asterisks). Solid lines: approximation of the experimental data by the function $\log \gamma=\gamma_{0}-\gamma_{1} v$. Curve 1: $\gamma_{0}=3.01$, $\gamma_{1}=1.04 \mathrm{E}-2$; curve $2: \gamma_{\mathrm{o}}=3.27, \gamma_{1}=5.68 \mathrm{E}-2$; curve $3: \gamma_{\mathrm{o}}=3.08, \gamma_{1}=3.28 \mathrm{E}-2$

Figure 6-49 reveals that the dimensionless parameter $\beta$ decreases with $v$ for all three melts. For any concentration of glass fibers, the highest value of $\beta$ is obtained for virgin PC and its lowest value is found for recycled PC. The rates of decrease in $\beta(v)$ are similar (within the accuracy of fitting observations) for all polymers. As the quantity $\beta$ characterizes the width of the distribution function $p$, the data depicted in Figure 18 show 
that the level of disorder in a transient network is higher for the recycled polymer than for virgin PC. This conclusion seems quite natural, because scission of macromolecules and creation of short chains induced by degradation of polycarbonate result in an increase of the heterogeneity of the equivalent network (that is reflected by the decrease in $\beta$ depicted in Figure 6-49).

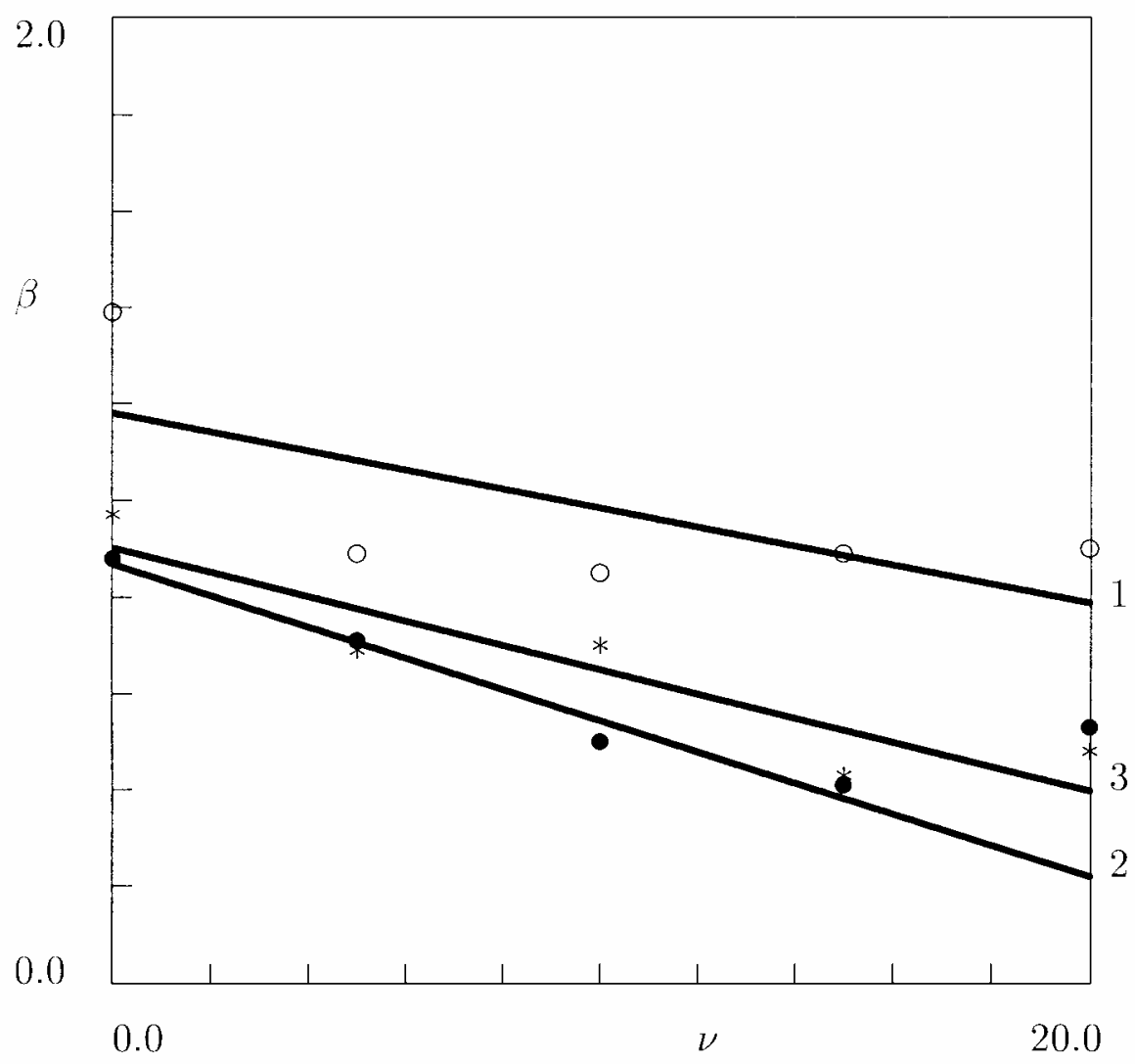

Figure 6-49: The dimensionless parameter $\beta$ versus the filler content $v$ wt.- $\%$. Symbols: treatment of observations at $250{ }^{\circ} \mathrm{C}$ on virgin PC (unfilled circles), recycled PC (filled circles) and the mixture of $50 \mathrm{wt.}-\%$ virgin with $50 \mathrm{wt.}$ - $\%$ recycled PC (asterisks). Solid lines: approximation of the experimental data by the function $\beta=\beta_{0}-\beta_{1} v$. Curve 1: $\beta_{0}=$ 1.18, $\beta_{1}=1.96 \mathrm{E}-2$; curve $2: \beta_{0}=0.87, \beta_{1}=3.24 \mathrm{E}-2$; curve $3: \beta_{0}=0.90, \beta_{1}=2.52 \mathrm{E}-2$ 
Three series of shear oscillatory tests have been performed on virgin polycarbonate, recycled polycarbonate, and their mixture reinforced with various amounts of short glass fibers, $v$, at two temperatures $\mathrm{T}_{1}=250$ and $\mathrm{T}_{2}=290{ }^{\circ} \mathrm{C}$. Material constants are determined by matching experimental data for the storage and loss moduli. Good agreement is demonstrated between the observations arid the results of numerical simulation.

The following conclusions have been drawn:

1. For virgin PC, the instantaneous shear modulus, G, increases with temperature, $T$, in accord with predictions of the classical theory of rubber elasticity. At the low temperature, $T_{1}$, the elastic modulus of virgin polycarbonate grows with the content of glass fibers, whereas at the high temperature, $\mathrm{T}_{2}, \mathrm{G}$ decreases with $v$. The difference in the effect of short glass fiber on the shear modulus is attributed to weakening of interactions between polymeric chains and filler particles. For recycled $\mathrm{PC}$ and mixture $\mathrm{PC}$, the elastic modulus, G, decreases with the content of glass fibers, $v$.

2. The attempt rate, $\gamma$, increases with temperature, $\mathrm{T}$, in agreement with predictions of the theory of thermally activated processes, and decreases with the content of short glass fiber, $v$. The latter is explained by the constrains on mobility of polymeric chains imposed by the presence of fibers. The rate of rearrangement, $\gamma$, for virgin PC exceeds that for recycled PC, which is associated with the presence of short chains in the recycled material (arising due to chemical degradation of a polymer) that serve as extra physical cross-links in the transient network. 
3. The average energy for rearrangement of strands, V, decreases with the content of short glass fiber, $v$. This observation is explained by the fact that the strength of entanglements between chains (the average energy of thermal fluctuations necessary for their disentanglement) noticeably exceeds the strength of interaction between chains and glass fibers. The rate of decrease in $\mathrm{V}$ with $v$ for recycled PC exceeds that for virgin PC, which is associated with better adhesion of chains to short glass fiber in virgin PC than in recycled PC.

\subsubsection{Dynamic Tan $\delta$}

The relationship between the storage and loss modulus can be represented in terms of the phase angle between the in-phase and out-phase portions of the modulus. The ratio of the loss modulus to the storage is defined as the tangent of the phase angle, $\delta$. The results for unfilled and filled polycarbonates are given in Figures 6-43, 6-44, and 645. The glass fibers increase the elastic component more than the viscous component at low frequency and less at higher frequency as demonstrated by $\tan \delta$ 


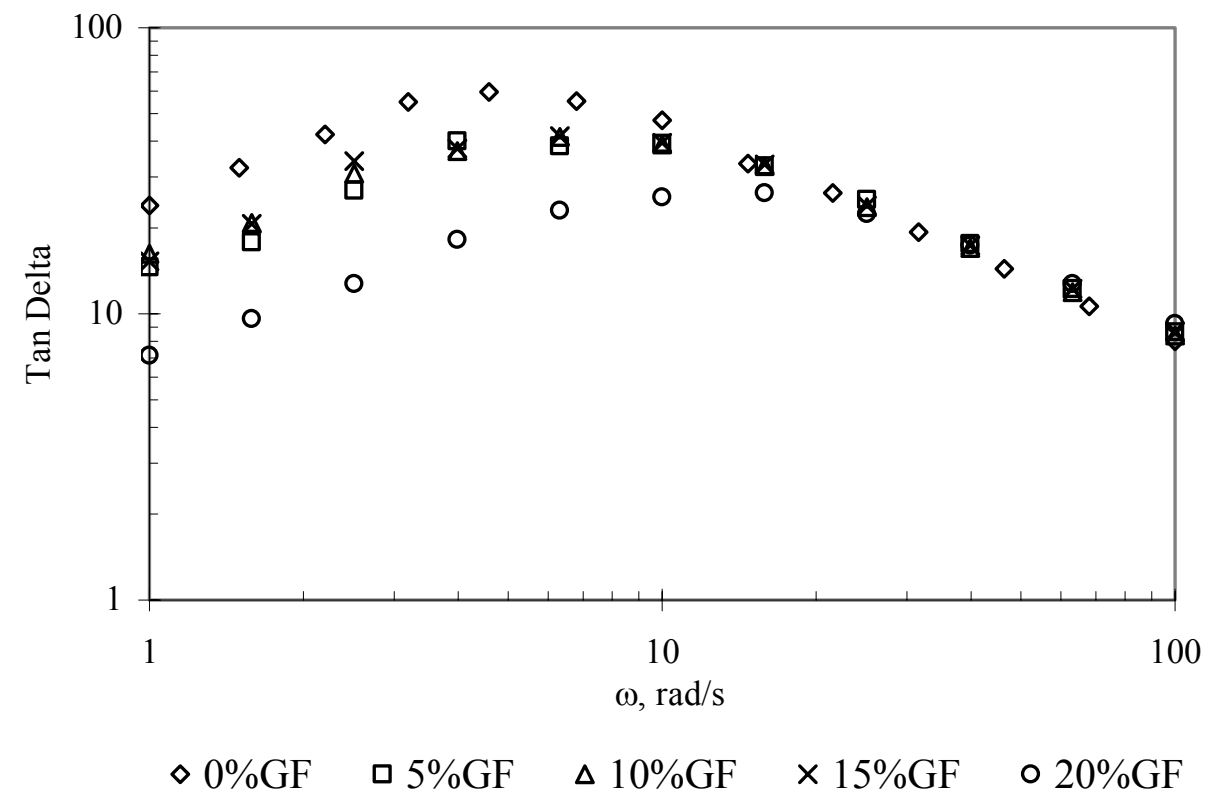

Figure 6-50 Virgin polycarbonate $\tan \delta$ vs. frequency

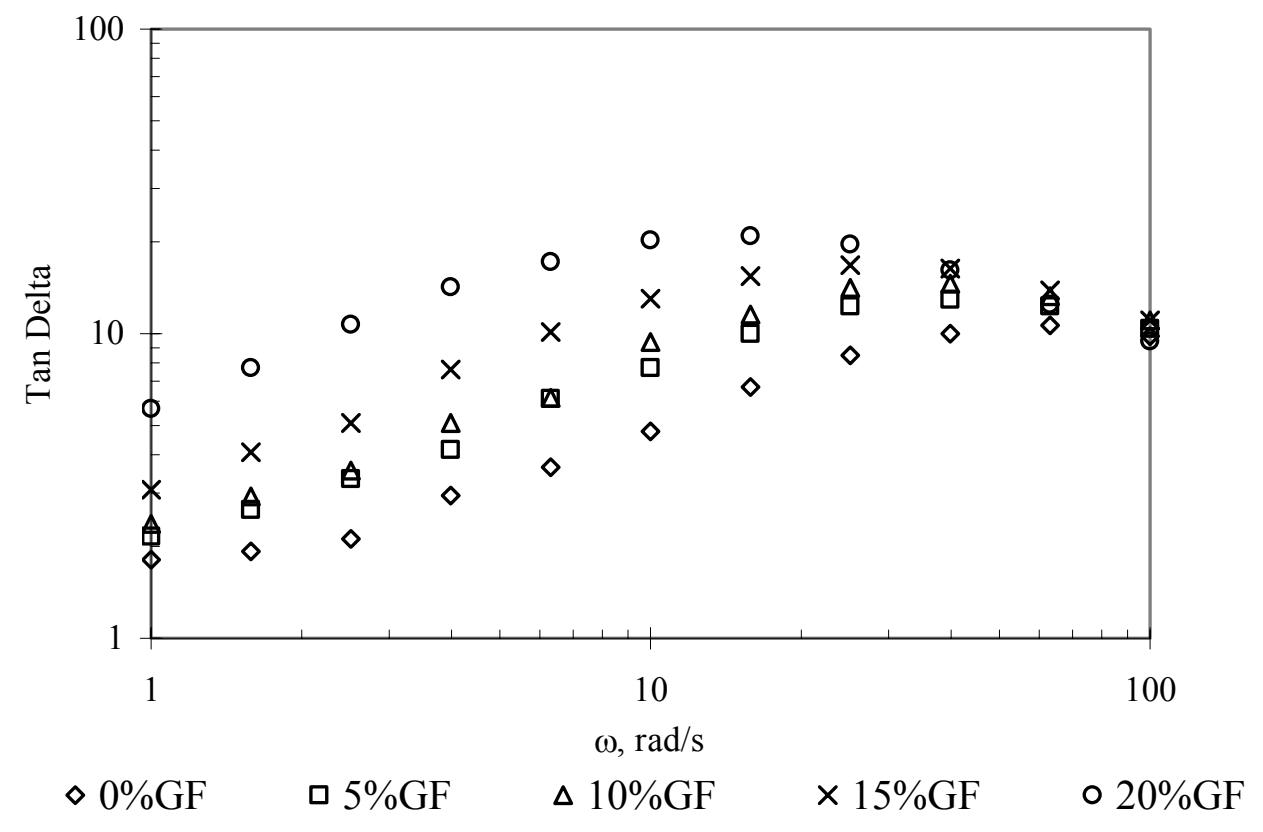

Figure 6-51 Recycled polycarbonate $\tan \delta$ vs. frequency 


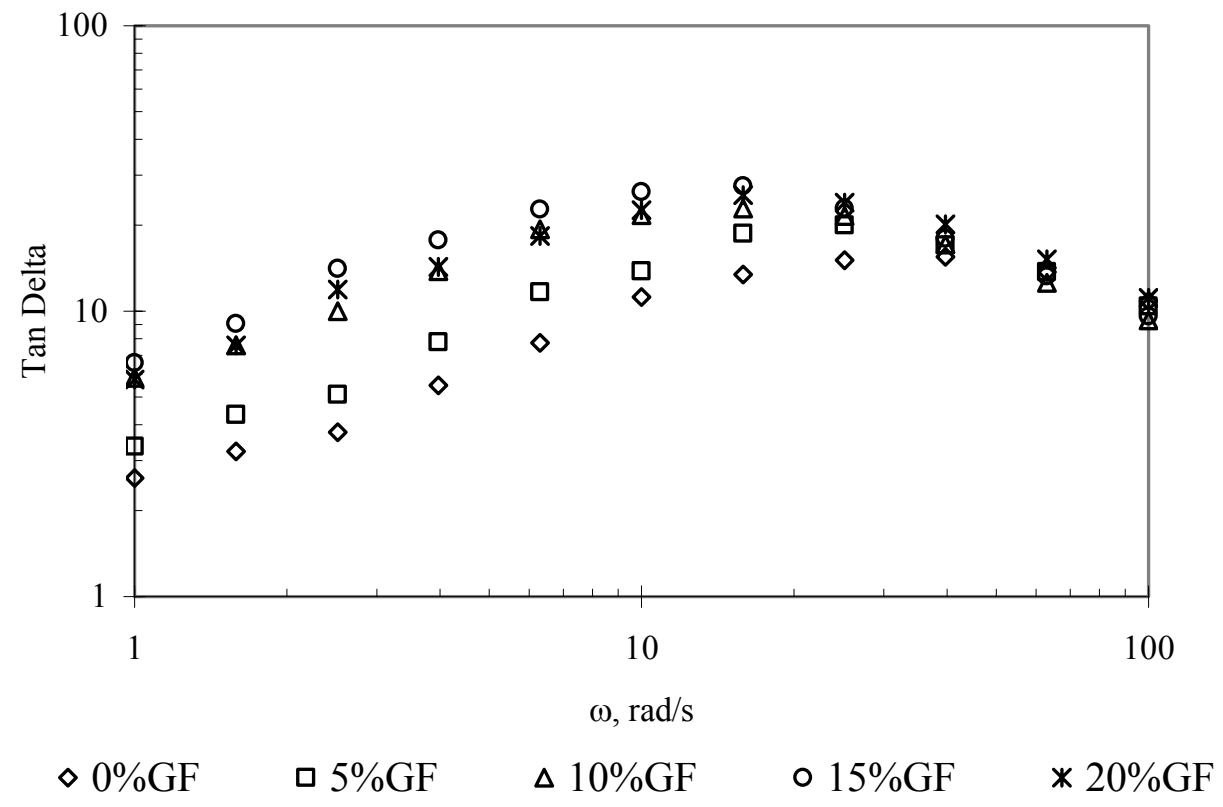

Figure 6-52 50\% virgin PC $+50 \%$ recycled $\mathrm{PC} \tan \delta$ vs. frequency

\subsubsection{Complex Viscosity}

The complex viscosity is defined as the ratio of complex modulus and rotation frequency. The complex viscosity has an in-phase and an out-phase viscosity, similar to that of the complex modulus. The complex viscosity for the unfilled and glass filled are given in Figures 6-46, 6-47, and 6-48. For all three materials, the short fibers significantly increase the viscosity. The unfilled recycled polycarbonate has a lower complex viscosity than the unfilled virgin polycarbonate, as seen in Figure 6-49. This is consistent with steady shear viscosity observation. When the glass fiber is added to the virgin and recycled polycarbonate the difference in complex viscosity is dramatically reduced, as seen in Figure 6-50. 


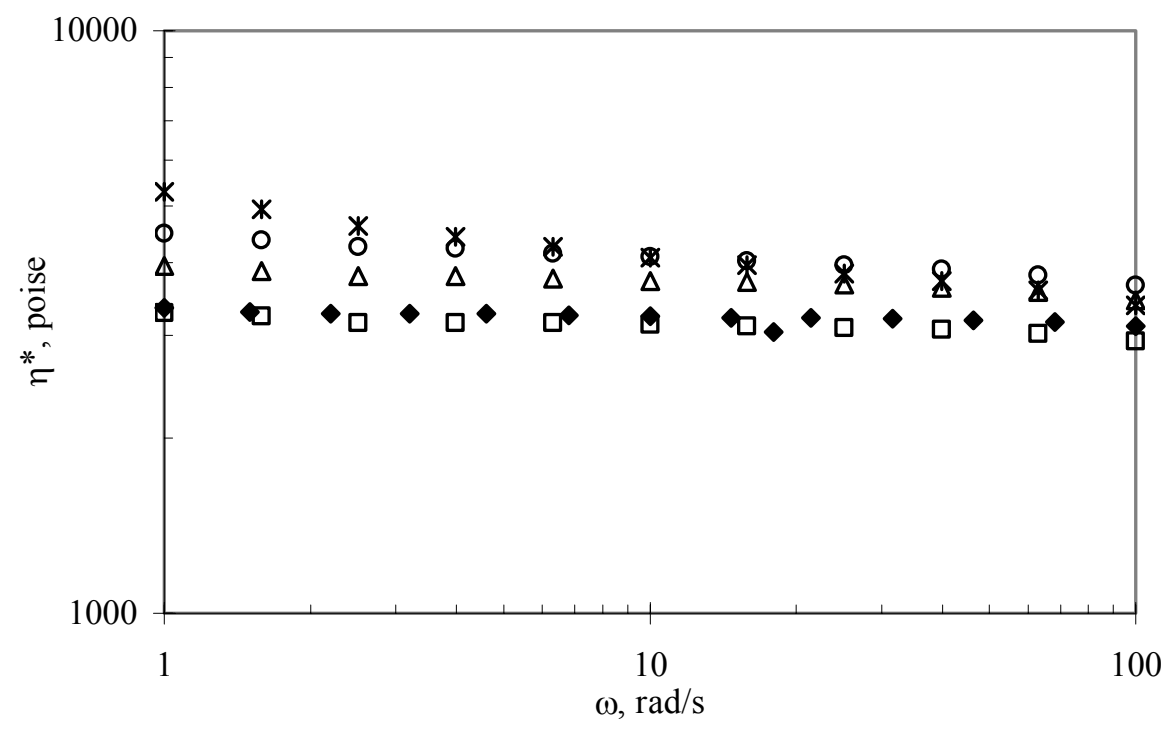

- $0 \% \mathrm{GF} \quad \square 5 \% \mathrm{GF} \quad \Delta 10 \% \mathrm{GF} \quad \circ 15 \% \mathrm{GF} \quad * 20 \% \mathrm{GF}$

Figure 6-53 Virgin polycarbonate complex viscosity vs. frequency $\left(\mathrm{T}=290{ }^{\circ} \mathrm{C}\right)$

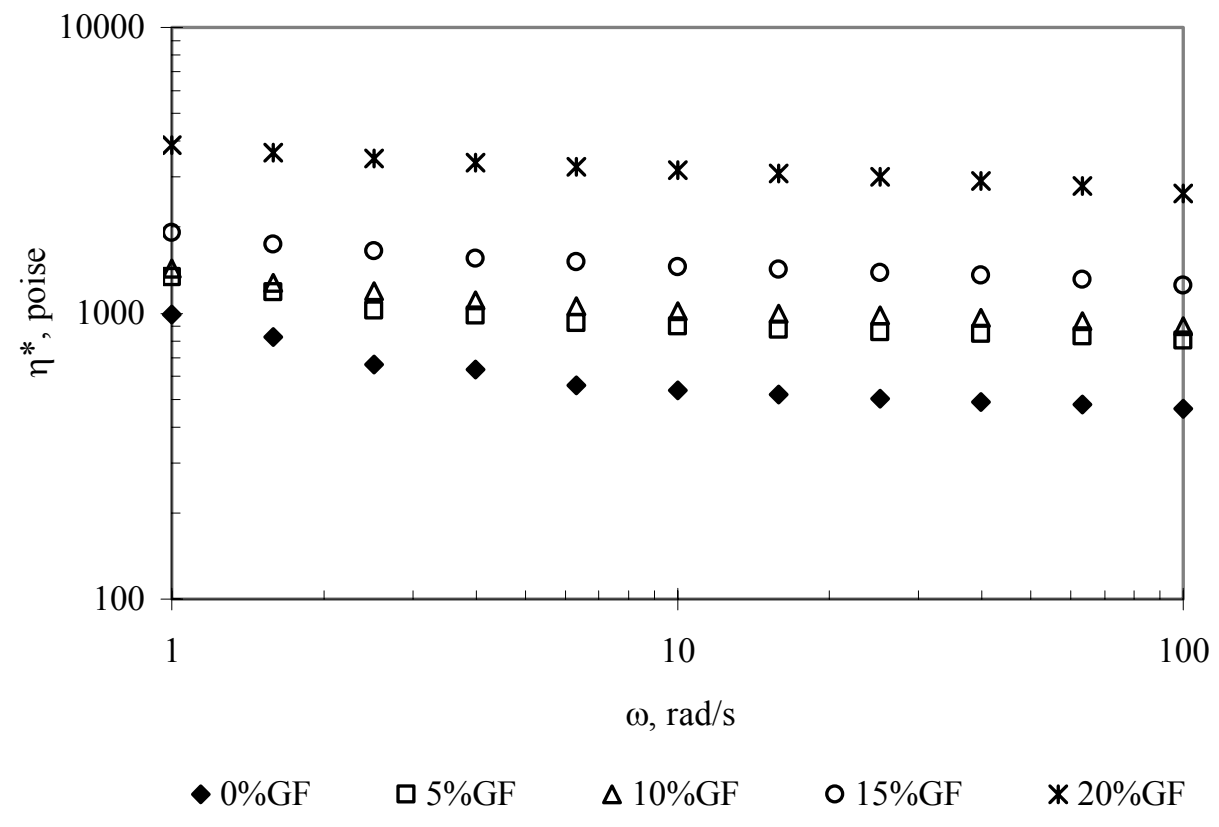

Figure 6-54 Recycled polycarbonate complex viscosity vs. frequency $\left(\mathrm{T}=290{ }^{\circ} \mathrm{C}\right)$ 


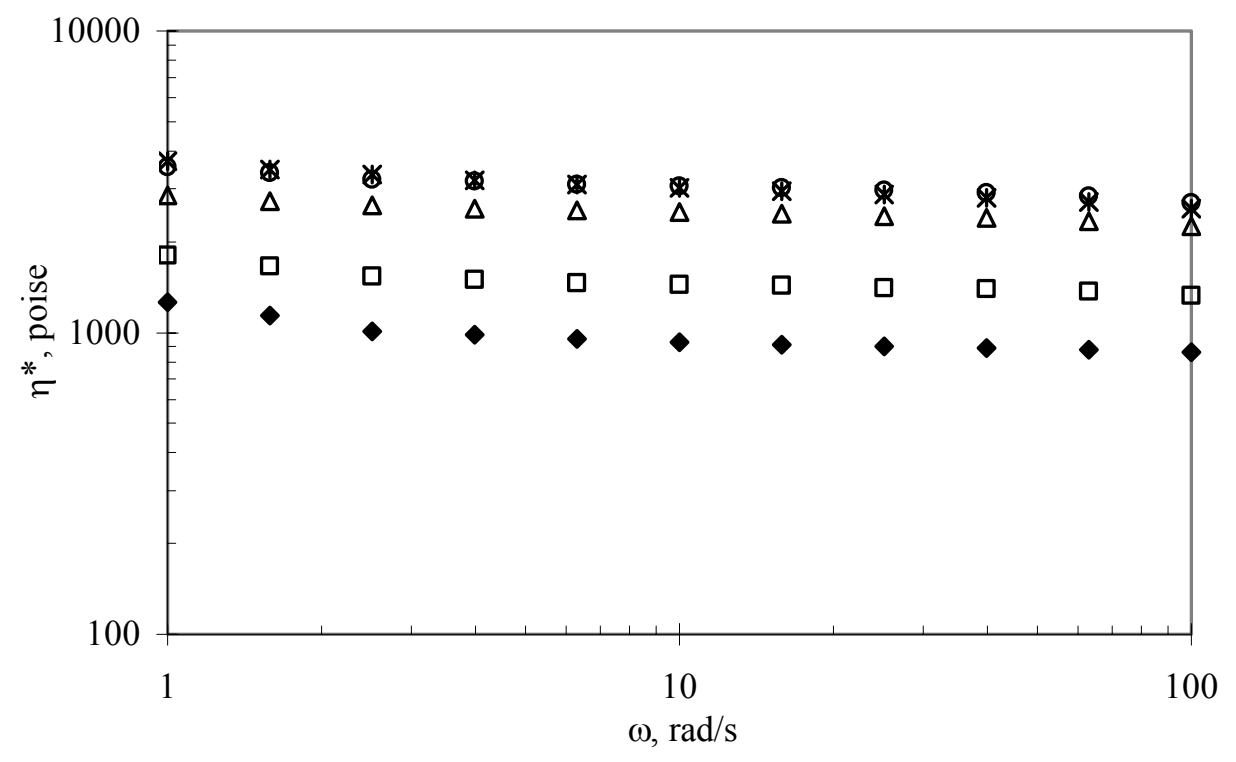

$\bullet 0 \% \mathrm{GF} \quad \square 5 \% \mathrm{GF} \quad \Delta 10 \% \mathrm{GF} \quad \circ 15 \% \mathrm{GF} \quad * 20 \% \mathrm{GF}$

Figure 6-55 50\% virgin polycarbonate $+50 \%$ recycled polycarbonate complex viscosity vs. frequency $\left(\mathrm{T}=290^{\circ} \mathrm{C}\right)$

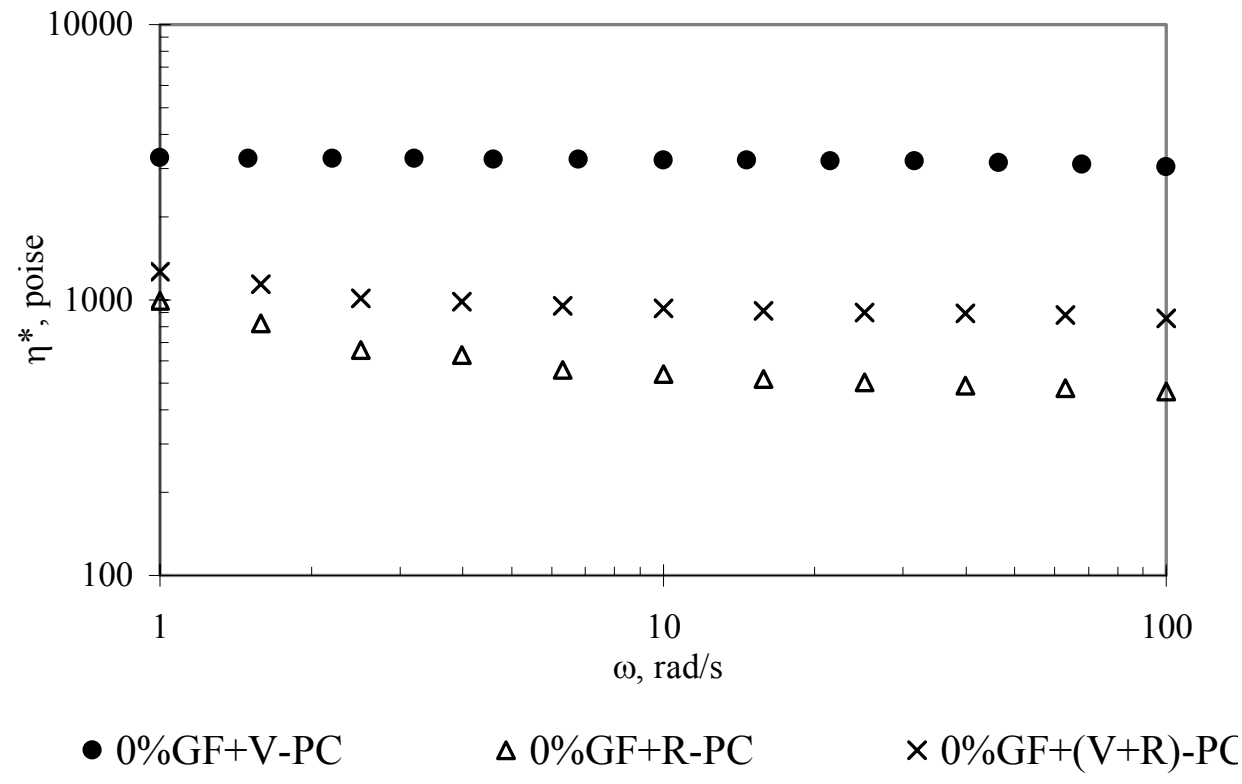

Figure 6-56 Unfilled polycarbonate complex viscosity vs. frequency $\left(\mathrm{T}=290{ }^{\circ} \mathrm{C}\right)$ 


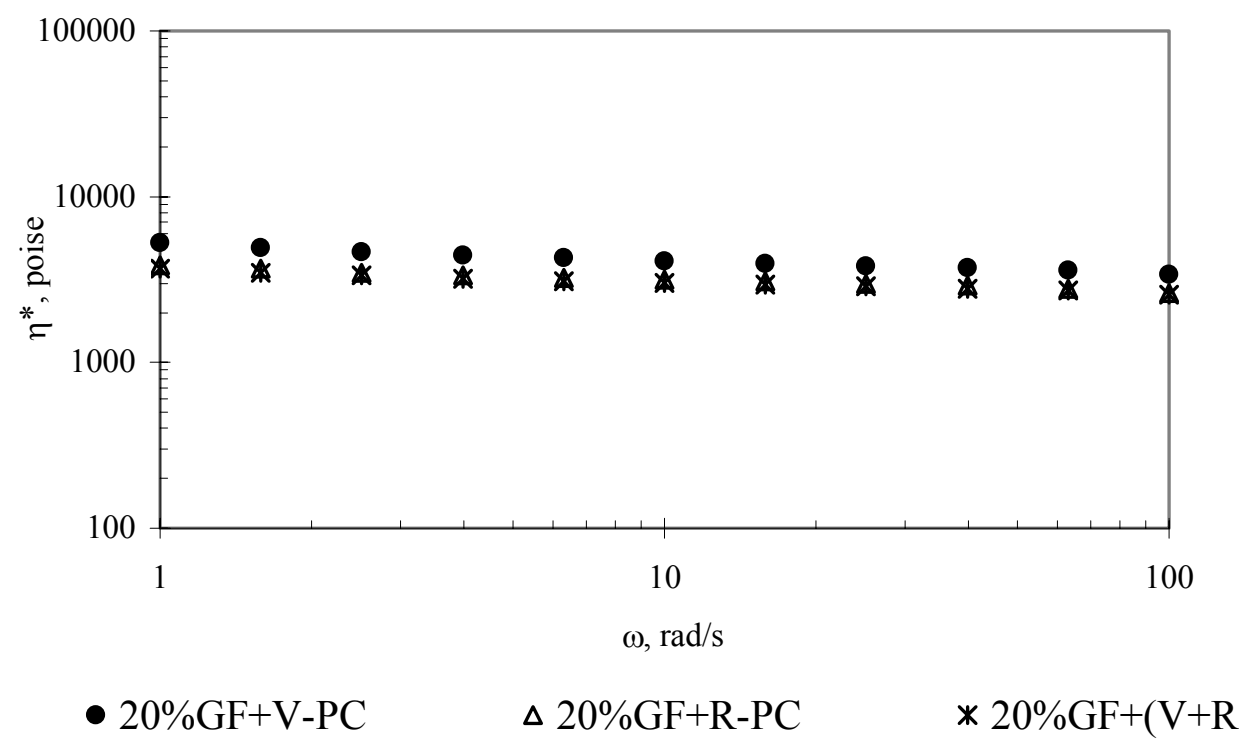

Figure 6-57 20\% glass fiber reinforced polycarbonate complex viscosity vs. frequency $\left(\mathrm{T}=290{ }^{\circ} \mathrm{C}\right)$

The viscosity of polymer melts changes dramatically with temperature. The variation is given by

$$
\frac{\Delta \eta}{\eta}=-\frac{\mathrm{E}}{\mathrm{RT}^{2}} \Delta \mathrm{T}
$$

Where $\mathrm{E}$ is the activation energy and $\mathrm{R}$ is the gas constant. The shape of the viscosityshear rate plots is hardly affected by temperature. The complex viscosity decreases as the temperature increases for $20 \%$ glass reinforced polycarbonate as seen in Figure 6-51. 


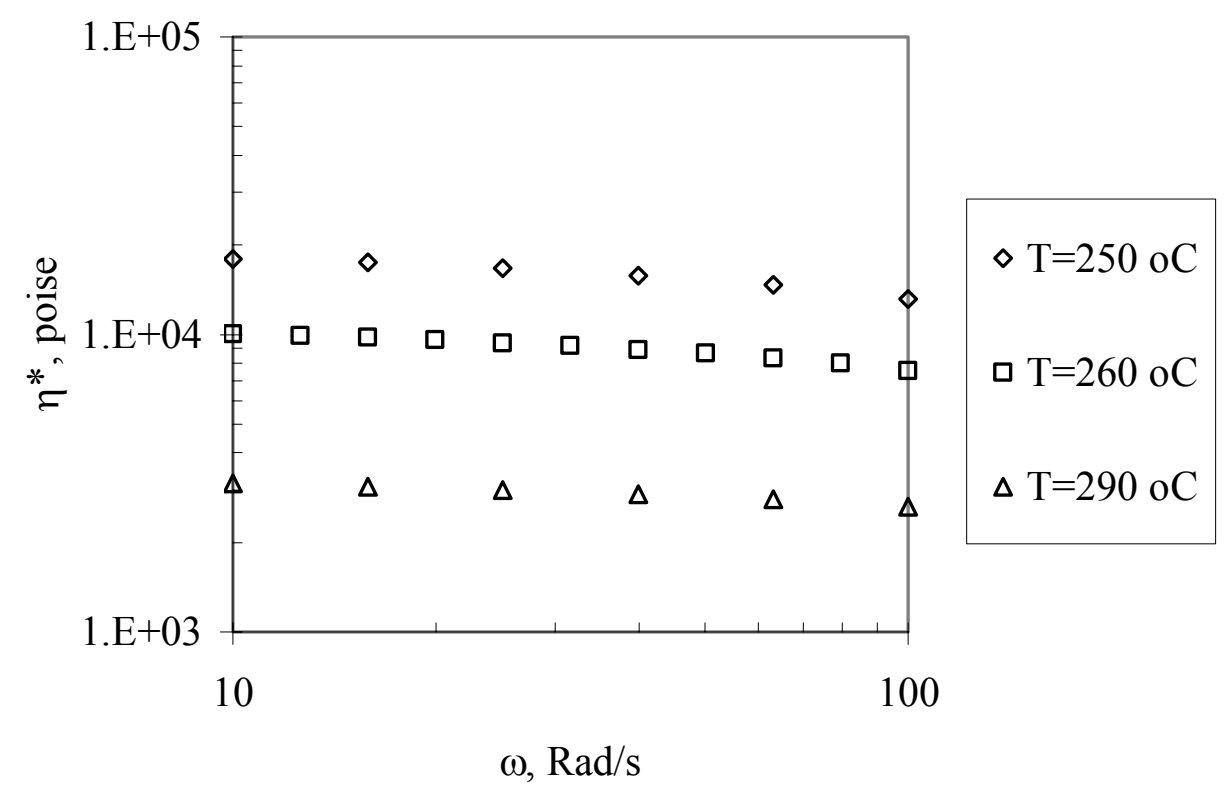

Figure 6-58 Influence of temperature on the complex viscosity of $20 \%$ glass reinforced recycled $\mathrm{PC}$ 


\section{Conclusions}

The primary goal of this work was to establish the effect of glass fibers on the mechanical, viscous, and elastic properties of the fiber-reinforced virgin, recycled, and mixture of virgin and recycled polycarbonate. The fibers had various lengths and the average length decreased as the amount of fibers increased. The dependence of various mechanical and fracture properties on the volume fraction, $\phi_{\mathrm{f}}$ of the reinforcing glass fiber in polycarbonate was investigated in tension and bending. Results indicate that the addition of glass fiber;

- Enhances ultimate tensile and flexural strengths of the polymer matrix. Variation for both strengths was linear with respect to $\phi_{\mathrm{f}}$ and thus obeying the rule of mixtures for strengths.

- Enhances the elastic modulus of the polymer. The elastic modulus was found to be a linear function of $\phi_{\mathrm{f}}$ which was subsequently described by the rule of mixtures.

- Reduces the elongations to yield and to break.

- Reduces the notched and un-notched impact properties of the polymer matrix. Recycled polycarbonate without the addition of glass fibers has poor mechanical properties compared to virgin polycarbonate. This limits the use of recycled polycarbonate in "high-end" injection molded products. When glass fiber was added to the recycled polycarbonate the mechanical properties were improved significantly, making it closer in quality to the virgin polycarbonate. The mechanical properties depend primarily on the glass fiber and not on the matrix. This was observed in virgin polycarbonate to which different amount of impurities were added. The mechanical 
properties of $15 \%$ glass-reinforced virgin polycarbonate containing up to $25 \%$ impurities were similar to those of $15 \%$ glass reinforced polycarbonate with no impurities. This indicates that there is no need for separating the recycled thermoplastics.

The fracture surface was fiber pull-out for glass reinforced virgin and recycled polycarbonate. The fibers were aligned in the flow direction in the injection molding for tensile samples. Recycled polycarbonate has less ductile fracture than the virgin polycarbonate.

The melt flow index of recycled polycarbonate is lower than the melt flow index of virgin polycarbonate. The addition of glass fiber reduces the difference in the melt flow index of recycled polycarbonate and virgin polycarbonate. In the steady-state experiments, viscous stresses increase proportionately according to the concentration of fibers. In dynamic testing, the fibers increase the viscous and elastic components, as measured by the complex viscosity and modulus. Similarly to the steady-state results, the fibers increase the viscous and elastic nature of the fiber-filled composite at low frequency and to a lesser extent at higher frequency. The fibers increase the elastic component more than the viscous component at low frequency and less at higher frequency as demonstrated by $\operatorname{Tan} \delta$.

The results show that overall, recycled polycarbonate has poor rheological properties compared to virgin polycarbonate. Use of recycled polycarbonate in the construction of high value injection-molded products is, therefore, not recommended. The addition of glass fiber to recycled polycarbonate significantly improves the mechanical and rheological properties however, and makes it suitable for use in all products where virgin polycarbonate would be otherwise be used. 


\section{Reference}

1. Leaversuch, R., "Recycling faces reality as bottom line looms," Modern Plastics, p. 48-50, July 1994.

2. U.S. Environmental Protection Agency, Characterization of Municipal Solid Waste in the United States: 1999 Update, Washington, D.C., 1999.

3. Siegler,T., "Collecting plastic bottles more efficiently," Resource Recycling, p. 27-42, September 1994.

4. King, R., "Plastic bottle recycling rate rises 21\%," Plastics News, p.1,June 5, 1995.

5. Woods, R., "APC, NAPCOR Studies Show Increases in Plastic Container Recycling Rates," Waste Ages Recycling Times, p.6, June, 1995.

6. Studt, T., "Polycarbonate Research Focuses on Flow, Alloys, and Recycling," R\&D Magazine, p.26-30, July 1994.

7. “CD Recycling Plant is Europe's First,” Modern Plastics, p.13, Sept. 1995.

8. Mapleston, P., "Auto sector's recycling goals keep plastics on hot seat," Modern Plastics, p. 48-58, May 1995.

9. Freitag, D., Grigo, U., Muller, P. R., and Nouvertne, W., "Polycarbonates," Polym. Eng. Sci. Encyl., 11, Wiley Interscience, New York, 1987.

10. Hostettler F. and Cox E.F., "Compositions containing polycarbonate plasticizers," U.S. Patent 3,305,605, 1964.

11. Campbell, R. W., Claesen, C.A., Vrooman, Fontana, T., Miller, K., Quinn, C., and Van Es, P., "Phenolic compound end capped polyester carbonate," U.S. Patent $5,321,1141994$.

12. Odian, G., "Principles of Polymerization," 2nd ed., Wiley Interscience, New York, 
1981.

13. Krim, H., and Schnell, H., "Thermoplastic aromatic polycarbonates and their manufacture", U.S. Patent 3,028,365, 1962.

14. G. Jen-Tau and W. Chun-Shan, "The interfacial polycarbonate reactions. I. Defining the critical process parameters," J. Appl. Polym. Sci. 44, p 849, 1992.

15. Okamoto, M., "Polycarbonate made optical article; bisphenol A endcapped with 4cumyl phenol," U.S. Patent 4,997,903, 1991.

16. Berry, G. C., Nomura, H., and Mayhan, K. G., "Dilute solution studies on a polycarbonate in good and poor solvents," J. Polym. Sci. A-2, 5, 1, 1967.

17. Tsuji, T., Norisuye, T., and Fujita, H., "Dilute solution of bisphenol A polycarbonate, Polym. J. 11, p. 558, 1975.

18. Sitaramaiah, G., "Polymer-solvent interactions of bisphenol A polycarbonate in different solvents," J. Polym. Sci. A3, p. 2743, 1965.

19. Binsack, R., Haupt, H., Vernaleken, N., and Reese, F., "Process for preparing polycarbonates," U.S. Patent 3,931,108, 1976.

20. Hashimoto, K., Namanishi, N., and Yamanishi, N., "Polycarbonate resin composition; blends with ultrahigh molecular weight polycarbonate to improve flow ability while maintaining mechanical properties and transparency,” U.S. Patent 5,264,498, 1993.

21. American Society for Testing and Materials, ASTM D 638.

22. Charles A. H., "Handbook pf Plastics, Elastomers, and Composites," McGraw-HILL, New York, 1996.

23. American Society for Testing and Materials, ASTM D 790.

24. Vincent., P. I., "Impact Tests and Service Performance of Thermoplastics." Plastics 
Institute, London, 1971.

25. American Society for Testing and Materials, ASTM D 1709.

26. ASTM D1822 and D2289, Annual ASTM Standards.

27. American Society for Testing and Materials, ASTM D 256.

28. American Society for Testing and Materials, ASTM D 2240.

29. Philipp, H. R., Legrand, D. G., Cole, H. S., and Liu, Y. S., “The optical properties of bisphenol A polycarbonate," J. Polym. Eng. Sci. 27, p.114, 1987.

30. Dutta, A., “On viscosity-melt flow index relationship,” Rheol. Acta, 23, p. 565, 1984.

31. Legrand, D. G., Bendler, J. T., "Handbook of Polycarbonate Science and Technology” Mrcel Dekker, Inc., New York, 2000.

32. Olagoke, O., “Handbook of Thermoplastics,” Marcel Dekker, Inc., New York, 1997.

33. Dobkowski, Z., "Influence of polydispersity and long chain branching on the melt viscosity of polycarbonate," Eur. Polym. J. 18, 1051, 1982.

34. Schnell, H., Chemistry and Physics of Polycarbonates, Wiely Interscience, New York, 1964.

35. Munjal, S., and Kao, C., Mathematical model and experimental investigation of polycarbonate pellet drying, Polym. Eng. Sci. 30, 1352, 1990.

36. Markezich, R. L., Thermally stable polycarbonate compositions comprising oxetane phosphonites, U.S. Patent 4,247,448, 1981.

37. Gupta, M. C., and Tahilyani, G. V., Photodegradation of poly(4,4'-isopropylidine diphenylene carbonate), Colloid Polym. Sci. 266, 620, 1988.

38. Gugumus, F., "Plastics Additives Handbook," 4th ed. Hanser, Cincinnati, 1993.

39. Pryde, C. A., Kelleher, P. G., Heilman, M. Y., and Wentz, R. P., "Hydrolytic 
stability of some commercially available polycarbonates," J. Polym. Eng. Sci. 22, $370,1982$.

40. GE Plastics, "Polycarbonate Processing Conditions," www.geplastics.com .

41. Hasushita, S., "Finder optical system in camera,” U.S. Patent 5,442,481, 1995.

42. Tennyson, P. C., "Prefabricated false tooth unit particular to provide pontic-has cap securable to adjacent tooth with flange fixable through replacement tooth side opening,” U.S. Patent 5,458,489, 1995.

43. Baumann, H. Eyelet especially for shower curtain is force-fitted tightly in curtain to reduce risk of tear out,” European Patent 673611, 1995.

44. Moor, G. R., Kline, D. E., "Properties and processing of Polymers for Engineers," Printice-Hall International, Inc., London, 1984.

45. Jones, R. F., "Guide to Short Fiber Reinforced Plastics," Hansser/Garder Publication, Inc., 1998.

46. Gibson, R. F., “ Principle of composites material mechanics,” R. R. Donnelley \& Sons Company, 1994.

47. Derek Hull, D. and Clyne T. W., "An introduction to composite material," Cambridge University Press, 1981.

48. Folkes, M. J., "Short Reinforced Thermoplastics," Research Studies Press, John Wiley \& Sons, 1982.

49. Cox, H. L., "The elasticity and strength of paper and other fibrous materials," Brit. J. Appl. Phys., 3, p. 72, 1952.

50. Stowell, E.Z. and Liu, T.S., "On the mechanical behavior of fiber-reinforced crystalline materials," J. Mech. Phys. Solids, 9, p. 242-260, 1961. 
51. Batchelor, G.K., "Slender-body Theory for Particles of Arbitrary Cross-section in Stokes Flow," J. Fluid Mech, 44, 3, p. 419, 1970.

52. Doi, M., Edwards, S.F., "Dynamics of Rod-like Molecules in Concentrated Solution; Part1,” J. of Chem. Soc. Faraday Trnls. II, 74, 3, 560, 1974.

53. Leal, L., Hinch, E., "Theoretical Studies of a Suspension of Rigid Particles affected by Brownian Couples," Rheol. Acta, 12, 127, 1973.

54. Mooney, M., "The Viscosity of a Concentrated Suspension of Spherical Particles," J. Colloid Sci., 6, 162, 1951.

55. Zeigel, K., "The Viscosity of Suspensions of Large, Nonspherical Particles in Polymer Fluids,” J. Colloid Sci., 34, 185, 1970.

56. Frankel, N., Acrivos, A., "On the Viscosity of a Concentrated Suspension of Solid Spheres," Chem. Eng. Sci., 22, 847, 1967.

57. Miles, J., Murty, N., Molden, G., “The Viscosity of Fiber Suspensions at Low Fiber Volume Fractions," Polymer Eng. Sci., 21, 1171, 1982.

58. Mewis, J., Metzner, A.," The Rheological Properties of Suspensions of Fibers in Newtonian Fluids Subjected to Extensional Deformation,” J. Fluid Mech., 62, 593, 1974.

59. Metzner, A., "Rheology of Suspensions in Polymeric Liquids," J. of Rheology, 60. 29, 739, 1985.

61. John M. D., Kurt, F. W, "Melt Rheology and Its Role in Plastics Processing Therory and Applications," Chapman \& Hall, New York, 1995.

62. Menard, K. P., "Dynamic Mechanical Analysis A Practical Introduction," CRC Press, 
Boca Raton, 1999.

63. Kelly, A., Tyson, W. R., J. Mech. Phys. Solids, 6, 13, 1965.

64. Bader, M. G., Collins, J. F., "The strength, Ductility and Failure of thermoplastics reinforced with short-glass fibers", Progress in Science and Engineering of Composite, 1982

65. Hull, D., "Introduction to composite materials", Cambridge University Press, Cambridge, 1981.

66. Gupta, R. K., “Polymer and Composites Rheology,” Marcel Dekker Inc., New York, 2000.

67. Bibbo, M., Dinh, S., Armstrong, R., "Shear Flow Properties of Semiconcentrated Fiber Suspensions”, J. Rheology, 29, 905, 1986

68. Ferry, J.D. "Viscoelastic Properties of Polymers.” Wiley, New York, 1980

69. Friedrich, C., Honerkamp, J., Weese, J. "New ill-posed problems in rheology", Rheol. Acta, 35, 186-193, 1996.

70. Baurngärtel, M., Winter, H.H. "Determination of discrete relaxation and retardation time spectra from dynamic mechanical data”. Rheol Acta 28, 511-519, 1989

71. Roths, T.. Maier, D., Friedrich, C., Marth, M., Horierkamp, J. "Determination of the relaxation time spectrum from dynamic moduli using an edge preserving regularization method". Rheol. Acta, 39, 163-173, 2000

72. Doi, M., Edwards, S.F., “The Theory of Polymer Dynamics”. Oxford Univ. Press, Oxford, 1986

73. Green, MS., Tobolsky, A.V., "A new approach to the theory of relaxing polymeric media”. J. Ghem. Phys. 14, 80-92, 1946 
74. Yaniamoto, M., "The visco-elastic properties of network structure. 1. General formalist”. J. Phys. Soc. Japan, 11, 413-421, 1956

75. Lodge, A. S. "Constitutive equations from molecular network theories for polymer solutions. Rheol. Acta. 7, 379-392, 1968

76. Everaers, R. "Viscosity, plasticity, and diffusion as examples of absolute reaction rate" J. Chem. Phys. 4, 283-291, 1936

77. Green, MS., Tobolsky, A.V., "A new approach to the theory of relaxing polymeric media". J. Chem. Phys. 14, 80-92, 1946 


\section{Appendix A}

Table A- 1 Properties of Lexan HF1110 *

\begin{tabular}{|c|c|c|c|}
\hline Property & Value & Units & Method \\
\hline Tensile strength at yield & 9000 & psi & ASTM D 638 \\
\hline Tensile strength at break & 9500 & psi & ASTM D 638 \\
\hline Tensile elongation at break & 120.0 & $\%$ & ASTM D 638 \\
\hline Flexural stress at yield & 13500 & psi & ASTM D 790 \\
\hline Flexural modulus & 335000 & psi & ASTM D 790 \\
\hline Izod impact, notched & 12.0 & $\mathrm{ft}-\mathrm{lb} / \mathrm{in}$ & ASTM D 256 \\
\hline Specific gravity, solid & 1.20 & - & ASTM D 792 \\
\hline Water absorption, 24 hours at $73 \mathrm{~F}$ & 0.100 & $\%$ & ASTM D 570 \\
\hline Melt Flow Rate, $300^{\circ} \mathrm{C} / 1.2 \mathrm{~kg}$ & 25.0 & $\mathrm{~g} / 10 \mathrm{~min}$ & ASTM D 1238 \\
\hline
\end{tabular}

* Data from GE Plastics 
Table A- 2 Properties of RTP $307^{*}$

\begin{tabular}{|c|c|c|c|}
\hline Property & Value & Units & Method \\
\hline Tensile strength & 21000 & psi & D-638 \\
\hline Tensile elongation & 2.6 & $\%$ & D-638 \\
\hline Tensile modulus & $1.7 \times 10^{6}$ & psi & D-638 \\
\hline Flexural strength & 32000 & psi & D-790 \\
\hline Flexural modulus & $1.6 \times 10^{6}$ & psi & D-790 \\
\hline Compressive strength & 22000 & psi & D-695 \\
\hline Hardness, Rockwell R & 119 & & D-785 \\
\hline Izod Impact, notched & 3 & $\mathrm{ft}-\mathrm{lb} / \mathrm{in}$ & D-256 \\
\hline Izod Impact, notched, un-notched & 16 & $\mathrm{ft}-\mathrm{lb} / \mathrm{in}$ & D-256 \\
\hline Specific gravity & 1.52 & - & D-792 \\
\hline Water absorption, $24 \mathrm{hrs}$ at $23^{\circ} \mathrm{C}$ & 0.07 & $\%$ & D-570 \\
\hline
\end{tabular}

* Data from RTP Company 
Table A-3 Fibers properties (Data from PPG)

\begin{tabular}{ll}
\hline \hline Specific Gravity & 2.59 \\
\hline Diameter, mm & 12 \\
Tensile strength, psi & 250000 \\
Modulus of elasticity, psi & 10500000 \\
Poisson's Ratio & 0.22 \\
Linear Coeff. of Thermal Expansion & $2.8-3.3 \times 10^{-6}$ \\
(25-300oC), in $/ \mathbf{i n} /{ }^{\mathbf{0}} \mathbf{F}$ & \\
\hline \hline
\end{tabular}




\section{Appendix B}

\section{Glass Fiber Size and Size Distribution}

The glass fiber length and size distribution is given in Figures . The fiber length distribution is been fitted using Maxwell statistical method:

$P=P_{o} L^{\alpha} e^{-\lambda L}$

where $\mathrm{P}$ is the probability, $\mathrm{L}$ is the fiber length, $\alpha$ and $\lambda$ are constants. The constants $\alpha$ and $\lambda$ are listed in Table B-1 for all three systems. Table B-1 also summarized the average fibers length and the standard deviation of all three systems.

Table B- 1 Statistical summery for the glass fiber size and model parameters

\begin{tabular}{|l|c|c|c|c|c|}
\hline \hline \%GF & Polycarbonate & $\begin{array}{c}\text { Average } \\
\text { Length }\end{array}$ & $\begin{array}{c}\text { Standard } \\
\text { Deviation }\end{array}$ & $\alpha$ & $\lambda$ \\
\hline 40 & RTP 307 & 235 & 144 & 1.56 & 0.001 \\
\hline 5 & Virgin & 176 & 104 & 1.53 & 0.014 \\
\hline 10 & Virgin & 172 & 109 & 0.97 & 0.011 \\
\hline 15 & Virgin & 161 & 90 & 1.79 & 0.017 \\
\hline 20 & Virgin & 156 & 91 & 1.68 & 0.016 \\
\hline 5 & Recycled & 176 & 105 & 1.53 & 0.014 \\
\hline 10 & Recycled & 171 & 106 & 1.13 & 0.012 \\
\hline 15 & Recycled & 163 & 96 & 1.49 & 0.015 \\
\hline 20 & Recycled & 155 & 92 & 1.61 & 0.017 \\
\hline 5 & V+R & 173 & 104 & 1.46 & 0.014 \\
\hline 10 & V+R & 172 & 107 & 1.12 & 0.012 \\
\hline 15 & V+R & 159 & 86 & 1.82 & 0.017 \\
\hline 20 & V+R & 155 & 92 & 1.68 & 0.017 \\
\hline \hline
\end{tabular}




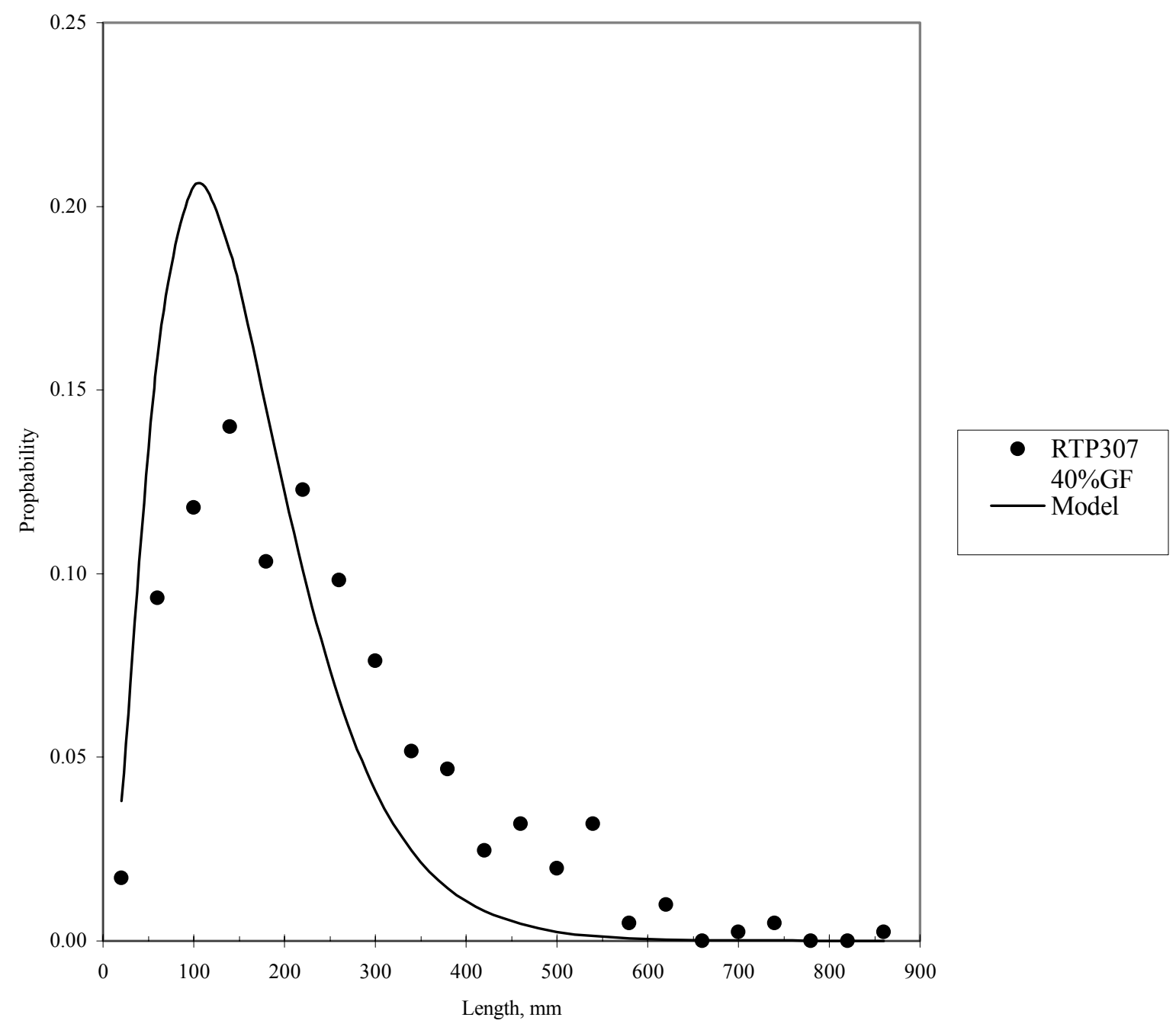

Figure B- 1 Glass fiber distribution for $40 \%$ glass-filled polycarbonate 


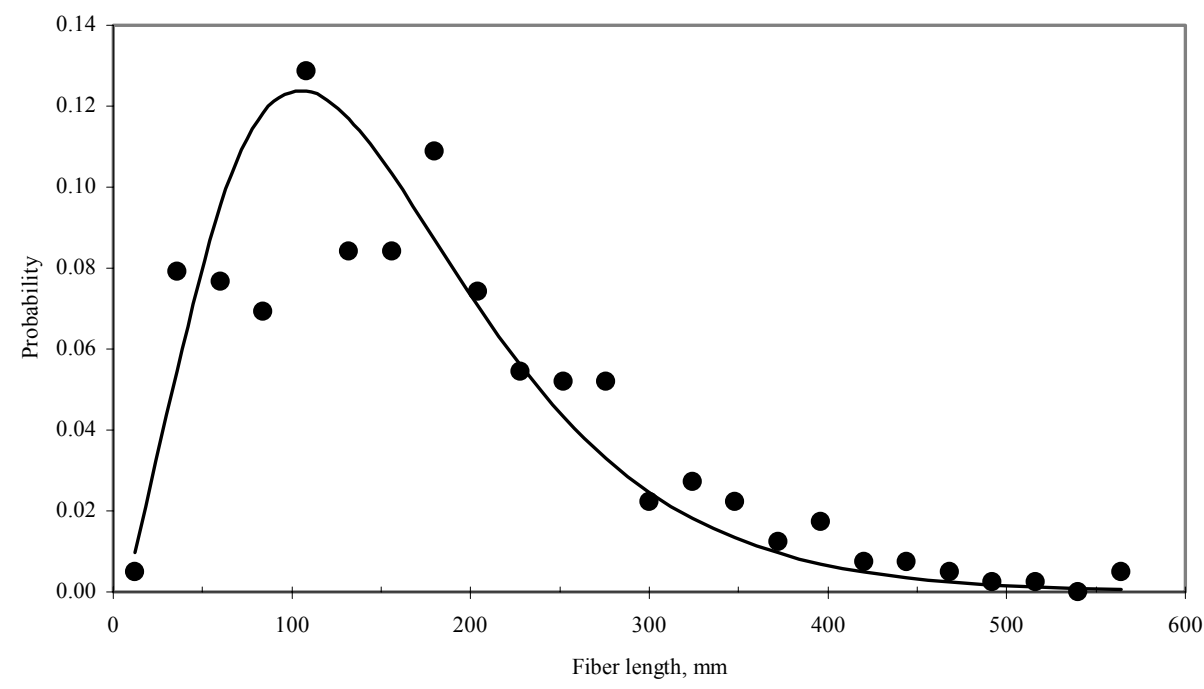

- $5 \% \mathrm{GF}-\mathrm{V}-\mathrm{PC}$ Model

Figure B- 2 Glass fiber distribution for 5\% glass-filled virgin polycarbonate

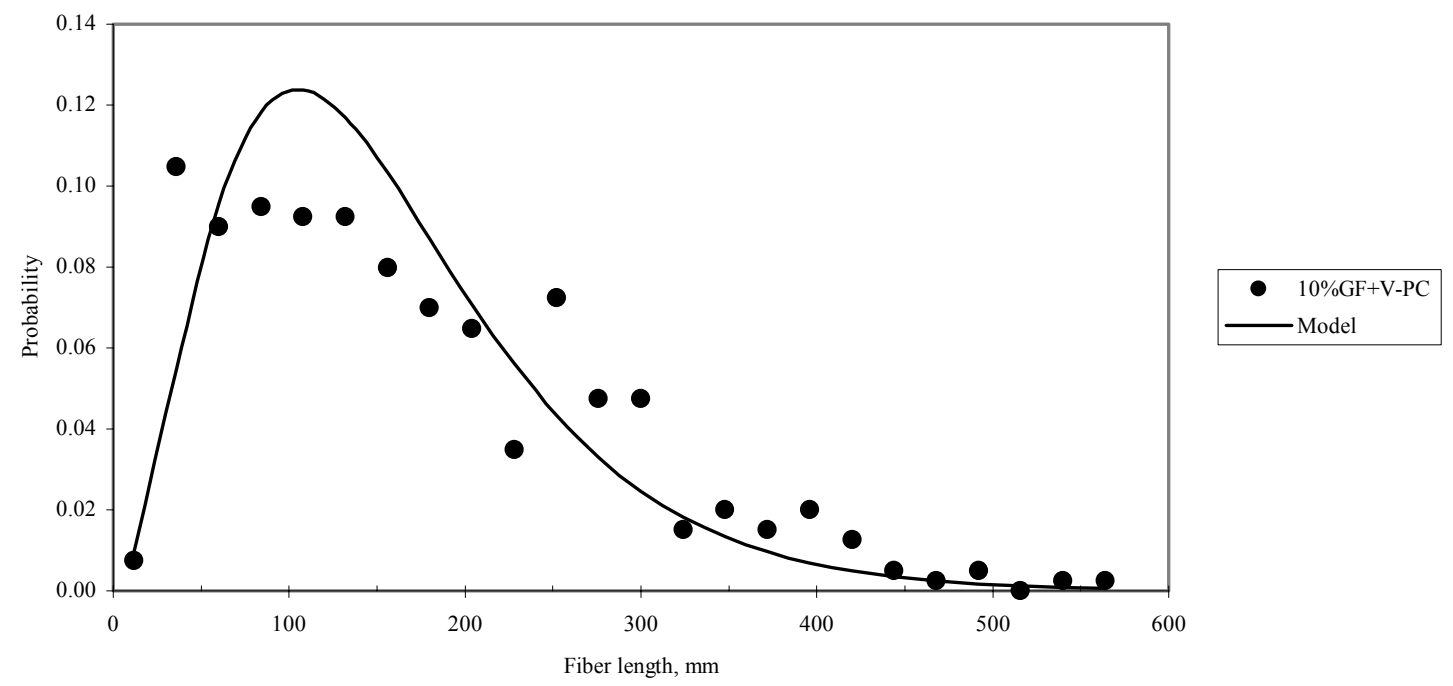

Figure B- 3 Glass fiber distribution for 10\% glass-filled virgin polycarbonate 


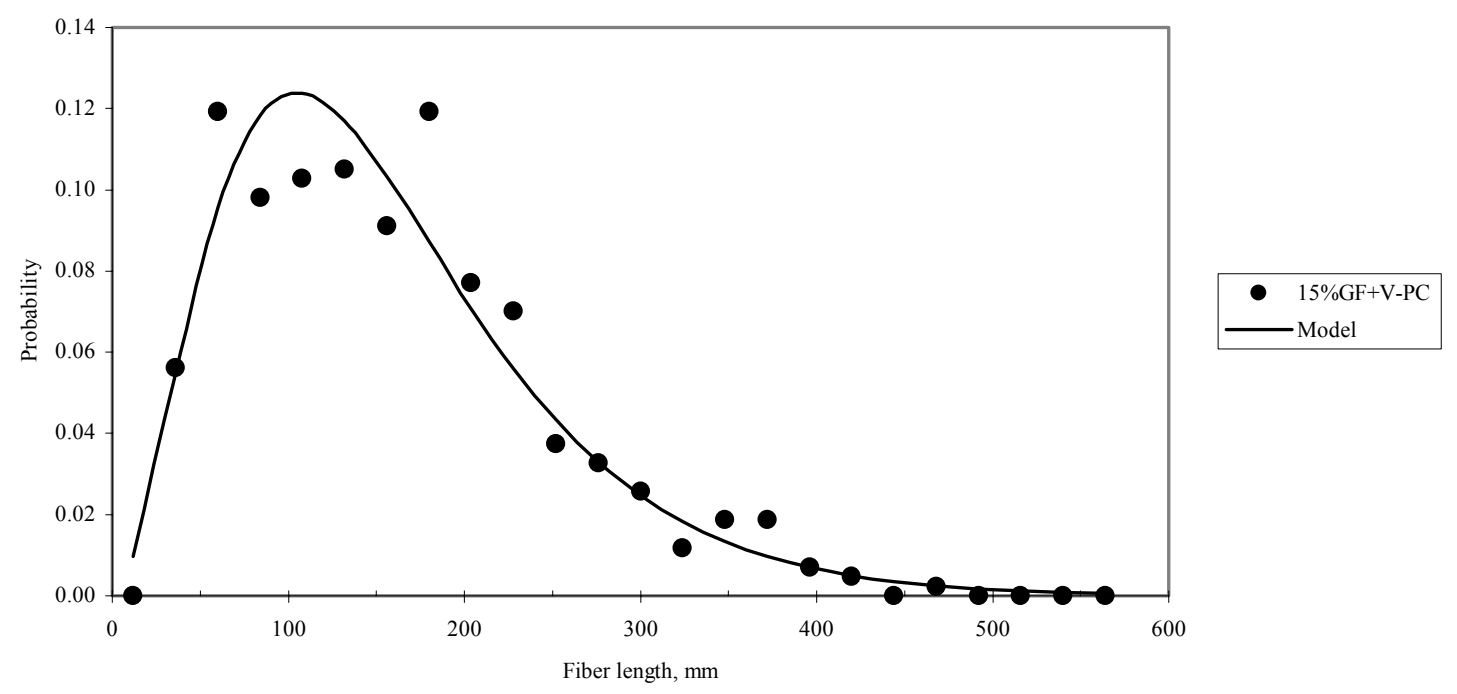

Figure B- 4 Glass fiber distribution for $15 \%$ glass-filled virgin polycarbonate

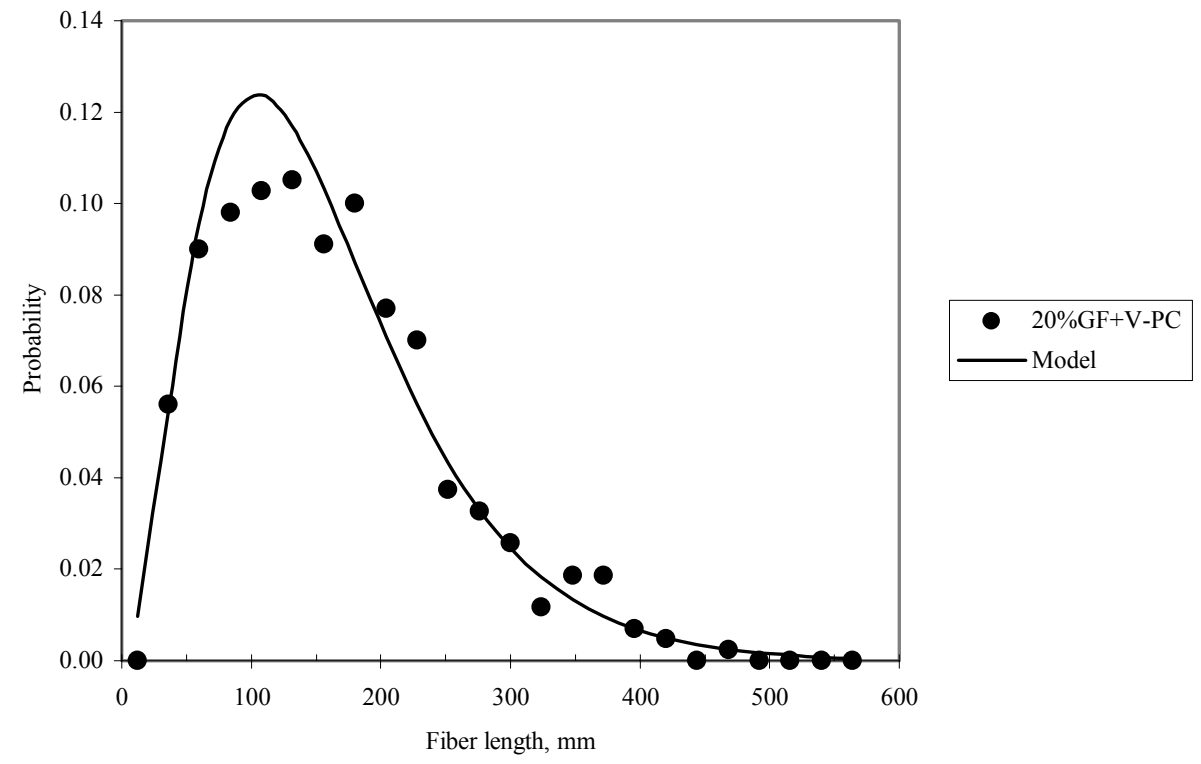

Figure B- 5 Glass fiber distribution for $20 \%$ glass-filled virgin polycarbonate 


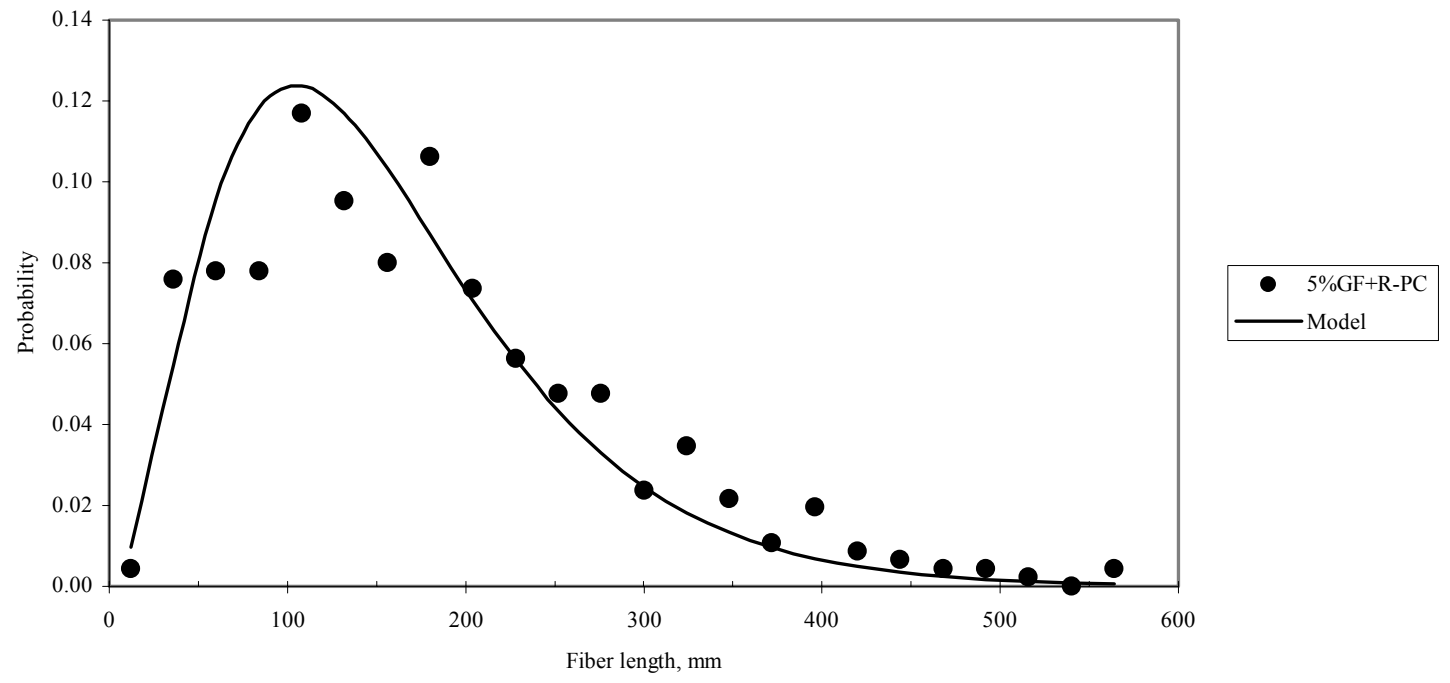

Figure B- 6 Glass fiber distribution for 5\% glass-filled recycled polycarbonate

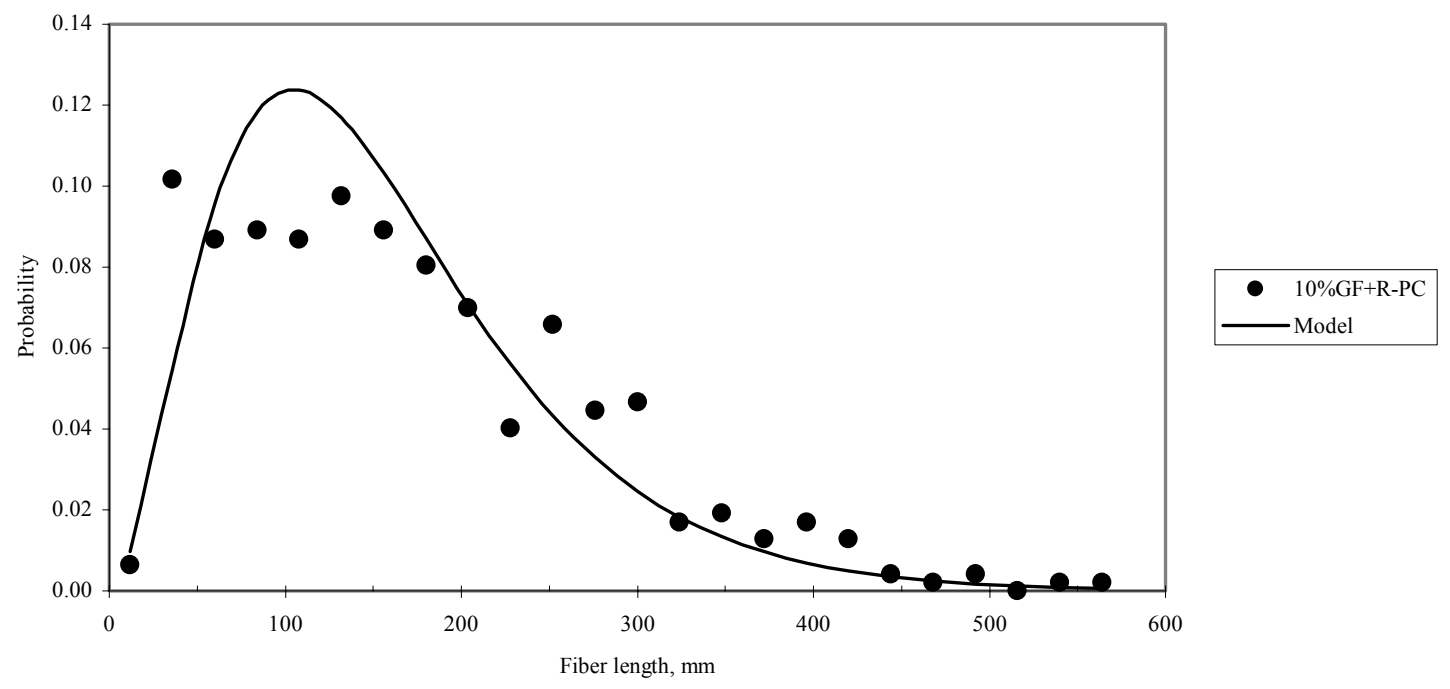

Figure B- 7 Glass fiber distribution for 10\% glass-filled recycled polycarbonate 


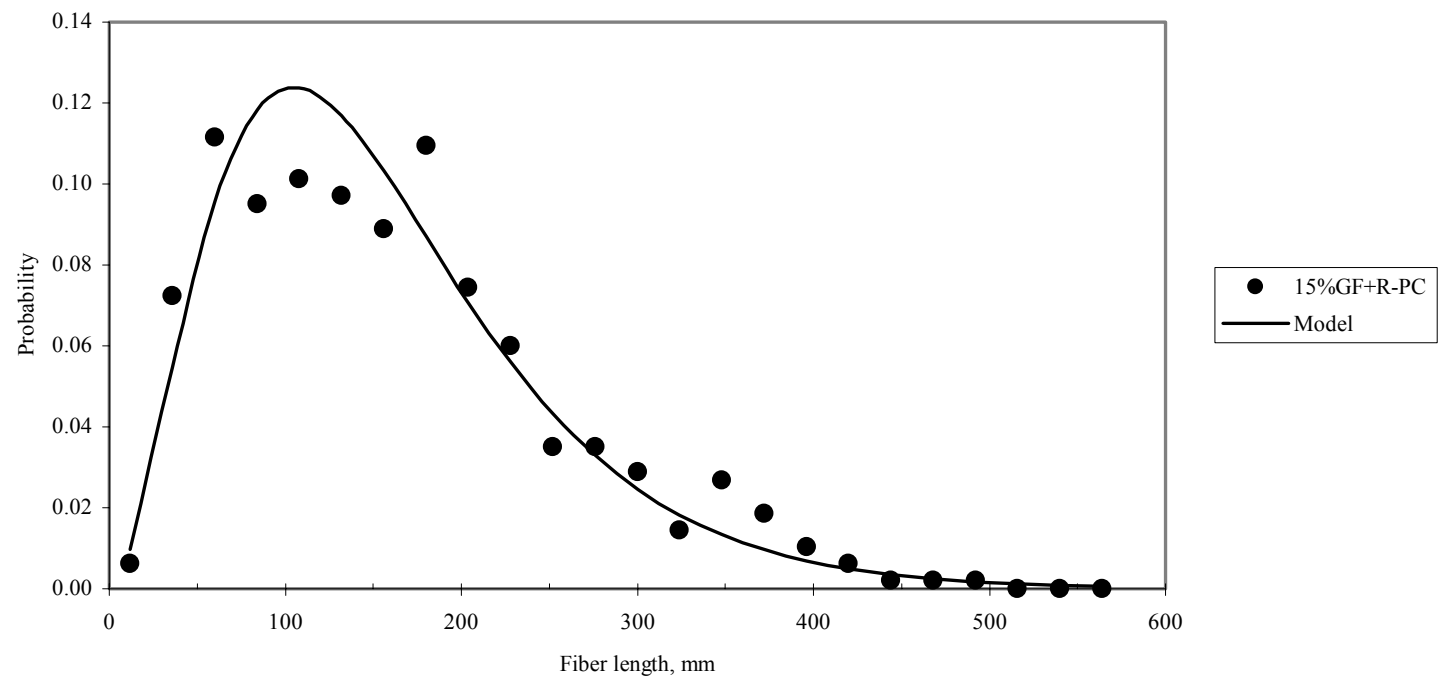

Figure B- 8 Glass fiber distribution for $15 \%$ glass-filled recycled polycarbonate

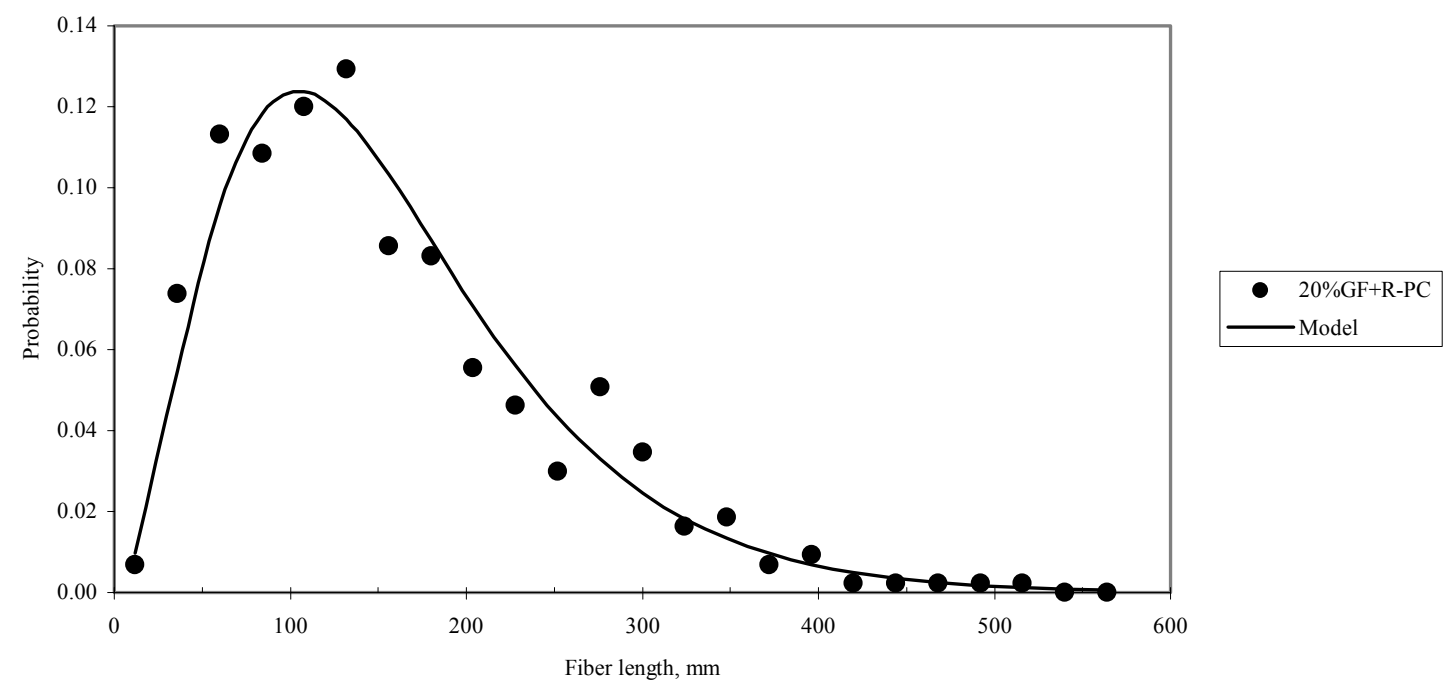

Figure B- 9 Glass fiber distribution for 20\% glass-filled recycled polycarbonate 


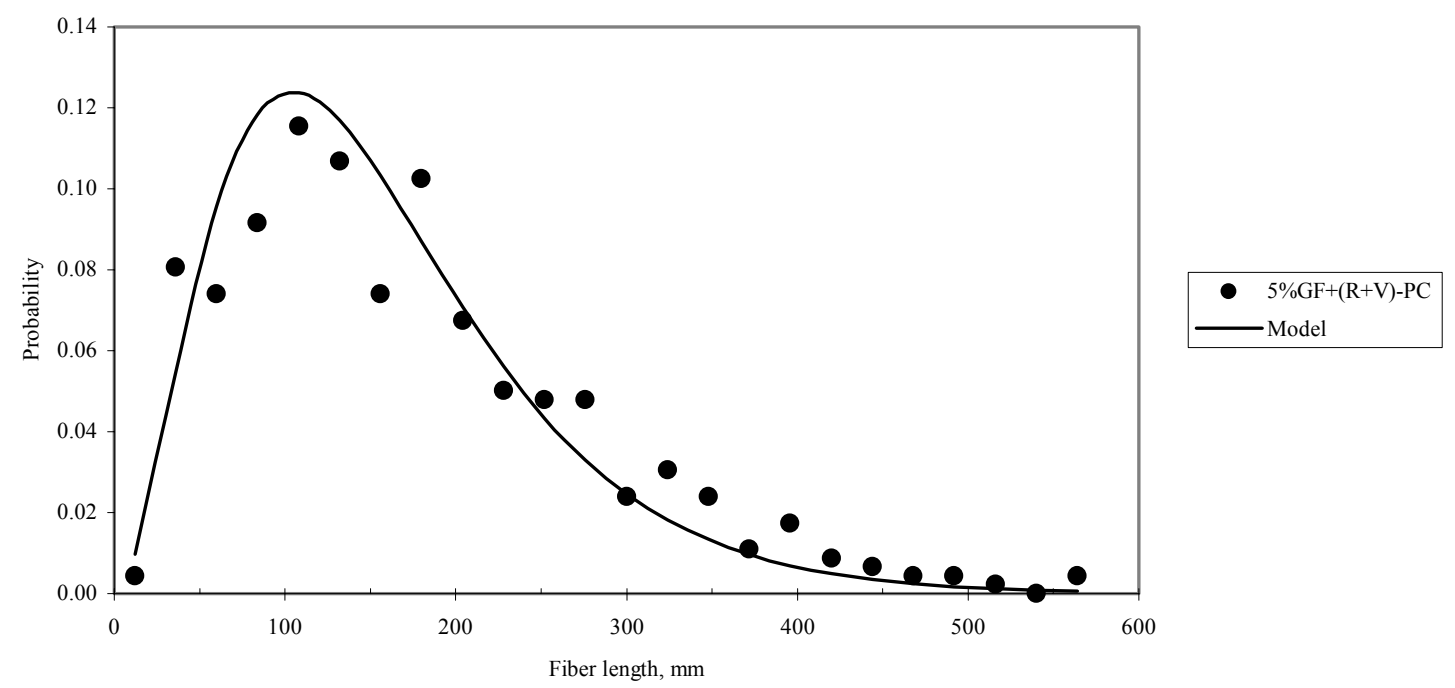

Figure B- 10 Glass fiber distribution for 5\% glass-filled (50\% virgin $+50 \%$ recycled) polycarbonate
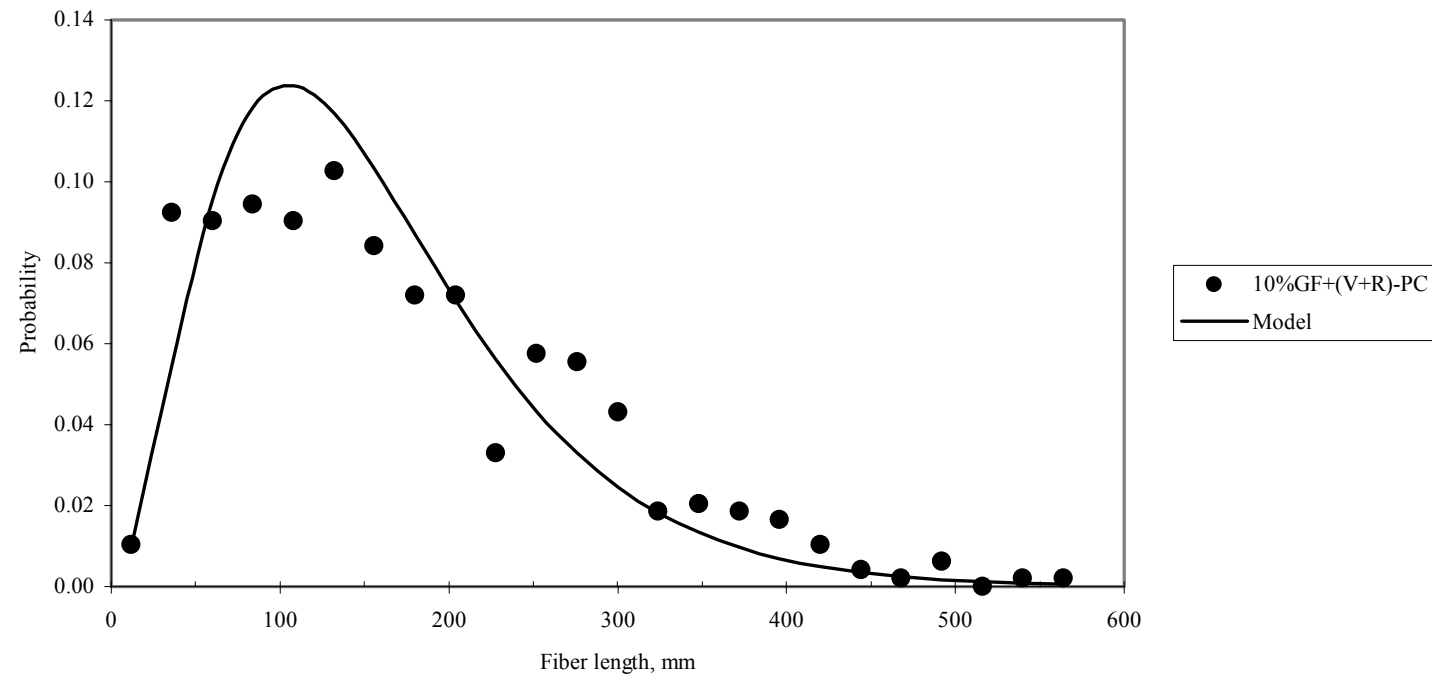

Figure B- 11 Glass fiber distribution for 5\% glass-filled (50\% virgin $+50 \%$ recycled) polycarbonate 


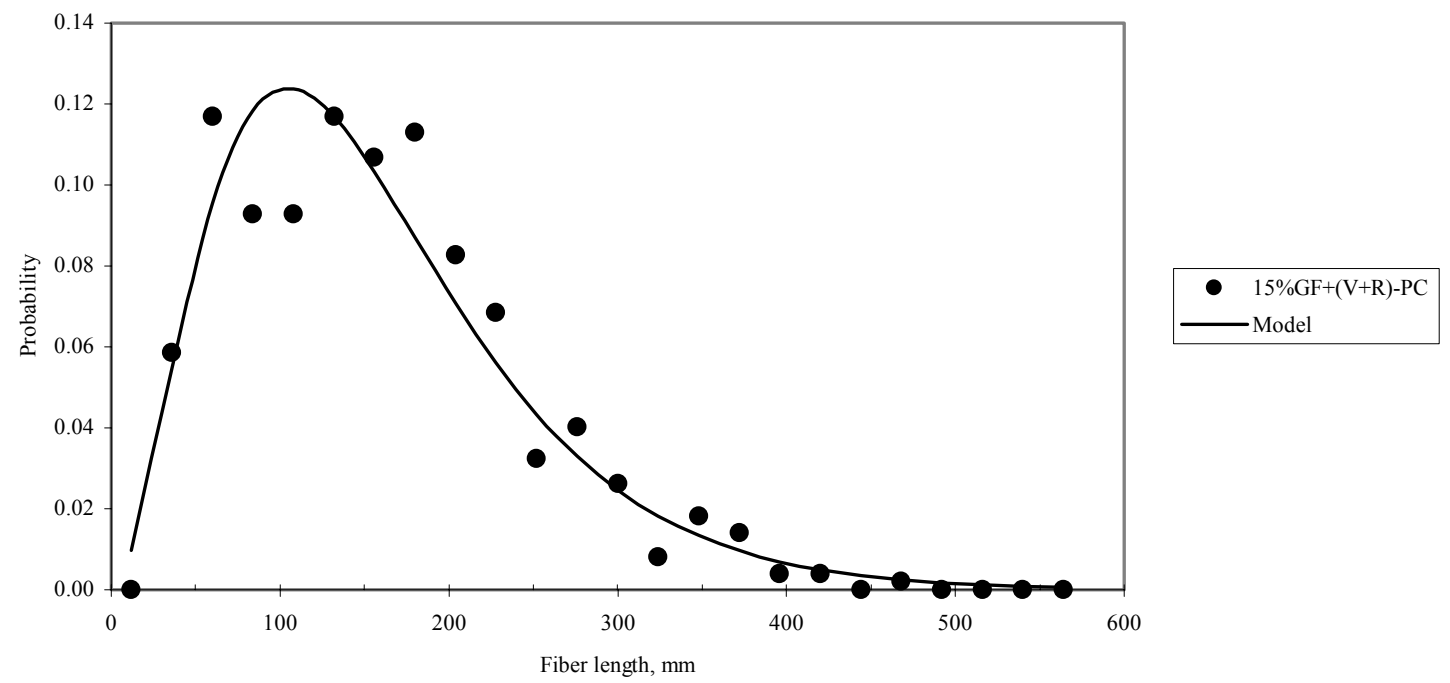

Figure B- 12 Glass fiber distribution for 5\% glass-filled (50\% virgin $+50 \%$ recycled) polycarbonate

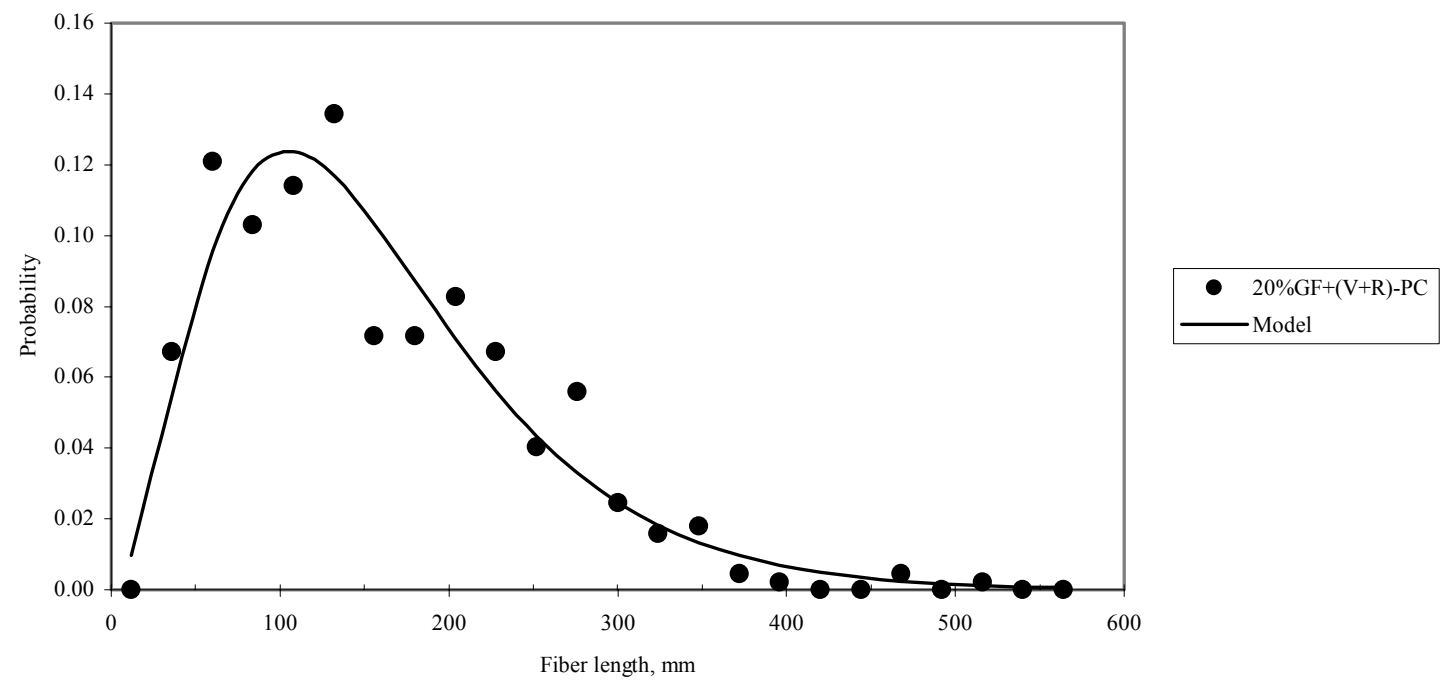

Figure B- 13 Glass fiber distribution for 5\% glass-filled (50\% virgin $+50 \%$ recycled) polycarbonate 


\section{Appendix C}

Detailed calculation for tensile strength using the rule of mixture:

$$
\begin{gathered}
\sigma_{\mathrm{c}}=\sigma_{\mathrm{f}} \phi_{\mathrm{f}} \eta_{\mathrm{L}} \eta_{\mathrm{o}}+\sigma_{\mathrm{m}}\left(1-\phi_{\mathrm{f}}\right) \\
\phi_{\mathrm{f}}=\left[1+\frac{\rho_{\mathrm{f}}}{\rho_{\mathrm{m}}}\left(\frac{1}{\mathrm{w}_{\mathrm{f}}}-1\right)\right]^{-1} \\
\eta_{\mathrm{L}}=\frac{\mathrm{L}_{\mathrm{f}}}{2 \mathrm{~L}_{\mathrm{c}}} \\
\mathrm{L}_{\mathrm{c}}=\frac{\mathrm{d} \sigma_{\mathrm{f}}}{2 \tau_{\mathrm{m}}} \\
\tau_{\mathrm{m}}=\frac{\sigma_{\mathrm{m}}}{2} \\
\sigma_{\mathrm{c}}=\sigma_{\mathrm{m}}+\phi_{\mathrm{f}} \sigma_{\mathrm{m}}\left[\frac{\mathrm{L}_{\mathrm{f}} \eta_{\mathrm{o}} \sigma_{\mathrm{f}}}{2 \mathrm{~L}_{\mathrm{c}} \sigma_{\mathrm{m}}}-1\right]
\end{gathered}
$$

The Slope of the line equals $\sigma_{m}\left[\frac{L_{f} \eta_{o} \sigma_{f}}{2 L_{c} \sigma_{m}}-1\right]$ from the slope one can calculate $\eta_{o}$

Tables below summarize the orientation and length efficiency for virgin, recycled, and blend of the virgin and recycled glass-reinforced polycarbonate. 
Virgin polycarbonate

\begin{tabular}{|c|l|c|c|l|c|c|c|}
\hline $\mathbf{w t} \%$ & $\begin{array}{l}\sigma_{\mathbf{m}}, \\
\mathbf{p s i}\end{array}$ & $\mathbf{L}_{\mathbf{f}}, \mu \mathbf{m}$ & $\begin{array}{l}\tau_{\mathbf{m}}, \mathbf{p s i} \\
\mathbf{u s i n g} \text { eq. } \\
\mathbf{( 6 )}\end{array}$ & $\begin{array}{l}\mathbf{L}_{\mathbf{c}}, \mu \mathbf{m} \\
\mathbf{u s i n g} \text { eq. } \\
\mathbf{( 5 )}\end{array}$ & $\begin{array}{l}\eta_{\mathbf{L}} \mathbf{u s i n g} \\
\mathbf{e q .}(\mathbf{3})\end{array}$ & $\begin{array}{l}\eta_{\mathbf{o}} \text { from } \\
\text { the slope }\end{array}$ & $\begin{array}{l}\sigma_{\mathbf{c}}, \mathbf{p s i} \\
\mathbf{u s i n g} \text { eq. } \\
\mathbf{( 1 )}\end{array}$ \\
\hline 5 & 8794 & 176 & 4397 & 341 & 0.26 & 0.50 & 9364 \\
\hline 10 & 8794 & 172 & 4397 & 341 & 0.25 & 0.51 & 9965 \\
\hline 15 & 8794 & 161 & 4397 & 341 & 0.24 & 0.54 & 10601 \\
\hline 20 & 8794 & 156 & 4397 & 341 & 0.23 & 0.56 & 11273 \\
\hline
\end{tabular}

\section{Recycled polycarbonate}

\begin{tabular}{|c|l|c|c|c|c|c|c|}
\hline $\mathbf{w t} \%$ & $\begin{array}{l}\sigma_{\mathbf{m}}, \\
\mathbf{p s i}\end{array}$ & $\mathbf{L}_{\mathbf{f}}, \mu \mathbf{m}$ & $\begin{array}{l}\tau_{\mathbf{m}}, \mathbf{p s i} \\
\mathbf{u s i n g} \text { eq. } \\
(\mathbf{6})\end{array}$ & $\begin{array}{l}\mathbf{L}_{\mathbf{c}}, \boldsymbol{\mu m} \\
\mathbf{u s i n g} \text { eq. } \\
(\mathbf{5})\end{array}$ & $\begin{array}{l}\eta_{\mathbf{L}} \mathbf{u s i n g} \\
\text { eq. (3) }\end{array}$ & $\begin{array}{l}\eta_{\mathrm{o}} \text { from } \\
\text { the slope }\end{array}$ & $\begin{array}{l}\sigma_{\mathbf{c}}, \mathbf{p s i} \\
\mathbf{u s i n g} \text { eq. } \\
(\mathbf{1})\end{array}$ \\
\hline 5 & 8991 & 176 & 4496 & 334 & 0.26 & 0.63 & 9793 \\
\hline 10 & 8991 & 171 & 4496 & 334 & 0.26 & 0.65 & 10640 \\
\hline 15 & 8991 & 163 & 4496 & 334 & 0.24 & 0.68 & 11534 \\
\hline 20 & 8991 & 155 & 4496 & 334 & 0.23 & 0.72 & 12479 \\
\hline
\end{tabular}


$\mathbf{5 0} \%$ virgin polycarbonate $+\mathbf{5 0} \%$ recycled polycarbonate

\begin{tabular}{|c|c|c|c|c|c|c|c|}
\hline wt\% & $\begin{array}{l}\sigma_{\mathrm{m}} \\
\text { psi }\end{array}$ & $\mathbf{L}_{\mathbf{f}}, \mu \mathbf{m}$ & $\begin{array}{l}\tau_{\mathrm{m}}, \text { psi } \\
\text { using eq. } \\
(6)\end{array}$ & $\begin{array}{l}\mathrm{L}_{\mathrm{c}}, \mu \mathrm{m} \\
\text { using eq. } \\
(5)\end{array}$ & $\begin{array}{l}\eta_{L} \text { using } \\
\text { eq. (3) }\end{array}$ & $\begin{array}{l}\eta_{\mathrm{o}} \text { from } \\
\text { the slope }\end{array}$ & $\begin{array}{l}\sigma_{c}, \text { psi } \\
\text { using eq. } \\
(1)\end{array}$ \\
\hline 5 & 8884 & 173 & 4442 & 338 & 0.26 & 0.62 & 9637 \\
\hline 10 & 8884 & 172 & 4442 & 338 & 0.25 & 0.62 & 10430 \\
\hline 15 & 8884 & 159 & 4442 & 338 & 0.24 & 0.67 & 11269 \\
\hline 20 & 8884 & 155 & 4442 & 338 & 0.23 & 0.69 & 12156 \\
\hline
\end{tabular}

Detailed calculation for modulus of elasticity using the rule of mixture

$$
\begin{aligned}
& E_{\mathrm{c}}=\mathrm{E}_{\mathrm{f}} \phi_{\mathrm{f}} \eta_{\mathrm{L}} \eta_{\mathrm{o}}+\mathrm{E}_{\mathrm{m}}\left(1-\phi_{\mathrm{f}}\right) \\
& \eta_{\mathrm{L}}=1-\frac{\tanh \mathrm{x}}{\mathrm{x}} \\
& \mathrm{x}=\frac{\mathrm{L}_{\mathrm{f}}}{2}\left(\frac{8 \mathrm{G}_{\mathrm{m}}}{\mathrm{E}_{\mathrm{f}} \mathrm{d}^{2} \ln (2 \mathrm{R} / \mathrm{d})}\right)^{1 / 2}
\end{aligned}
$$

$\mathrm{G}_{\mathrm{m}}$ : is shear modulus of the matrix which can be estimated

$$
\mathrm{G}_{\mathrm{m}}=\frac{\mathrm{E}_{\mathrm{m}}}{2\left(1+\mathrm{v}_{\mathrm{m}}\right)}
$$

$v_{\mathrm{m}}$ : The Poisson's ratio which equals to 0.35 for glass reinforced polycarbonate

2R: Center to center spacing of the fibers which for hexagonal packing arrangement 


$$
\frac{\mathrm{d}}{2 \mathrm{R}}=\left(\frac{2(3)^{1 / 2} \phi_{\mathrm{f}}}{\pi}\right)^{1 / 2}
$$

Tables below summarize the orientation and length efficiency for virgin, recycled, and blend of the virgin and recycled glass reinforce polycarbonate.

\section{Virgin polycarbonate}

\begin{tabular}{|c|c|c|c|c|l|l|l|l|}
\hline $\mathbf{w t} \%$ & $\mathbf{E}_{\mathbf{m}}, \mathbf{p s i}$ & $\begin{array}{l}\mathbf{L}_{\mathbf{f}}, \\
\boldsymbol{\mu m}\end{array}$ & $\begin{array}{l}\mathbf{G}_{\mathbf{m}}, \mathbf{p s i} \\
\mathbf{u s i n g} \mathbf{e q} . \\
\mathbf{( 1 1 )}\end{array}$ & $\begin{array}{l}\mathbf{d} / \mathbf{2 R} \\
\mathbf{u s i n g} \\
\mathbf{e q .}(\mathbf{1 2})\end{array}$ & $\begin{array}{l}\mathbf{x} \\
\mathbf{u s i n g} \\
\mathbf{e q .} \\
\mathbf{( 1 0 )}\end{array}$ & $\begin{array}{l}\eta_{\mathbf{L}} \\
\mathbf{u s i n g} \\
\mathbf{e q .} \text { (9) }\end{array}$ & $\begin{array}{l}\eta_{\mathrm{o}} \\
\text { from } \\
\text { the } \\
\text { slope }\end{array}$ & $\begin{array}{l}\mathbf{E}_{\mathbf{c}}, \mathbf{p s i} \\
\mathbf{u s i n g} \text { eq. } \\
\mathbf{( 8 )}\end{array}$ \\
\hline 5 & 302938 & 176 & 112199 & 0.16 & 1.58 & 0.42 & 0.80 & 381508 \\
\hline 10 & 302938 & 172 & 112199 & 0.24 & 1.74 & 0.46 & 0.72 & 464387 \\
\hline 15 & 302938 & 161 & 112199 & 0.29 & 1.77 & 0.47 & 0.71 & 551941 \\
\hline 20 & 302938 & 156 & 112199 & 0.34 & 1.84 & 0.48 & 0.69 & 644576 \\
\hline
\end{tabular}

\section{Recycled polycarbonate}

\begin{tabular}{|c|c|c|c|c|c|c|c|c|}
\hline$w t \%$ & $\mathbf{E}_{\mathbf{m}}, \mathbf{p s i}$ & $\begin{array}{l}\mathbf{L}_{\mathrm{f}} \\
\mu \mathbf{m}\end{array}$ & $\begin{array}{l}\mathbf{G}_{\mathrm{m}}, \mathbf{p s i} \\
\text { using eq. } \\
\text { (11) }\end{array}$ & $\begin{array}{l}d / 2 R \\
\text { using } \\
\text { eq. (12) }\end{array}$ & $\begin{array}{l}x \\
\text { using } \\
\text { eq. } \\
\text { (10) }\end{array}$ & $\begin{array}{l}\eta_{L} \\
\text { using } \\
\text { eq. (9) }\end{array}$ & $\begin{array}{l}\eta_{\mathrm{o}} \\
\text { from } \\
\text { the } \\
\text { slope }\end{array}$ & $\begin{array}{l}E_{c}, \text { psi } \\
\text { using eq. } \\
(8)\end{array}$ \\
\hline 5 & 338237 & 176 & 125273 & 0.16 & 1.69 & 0.45 & 0.87 & 430925 \\
\hline 10 & 338237 & 171 & 125273 & 0.24 & 1.83 & 0.48 & 0.81 & 528697 \\
\hline 15 & 338237 & 163 & 125273 & 0.29 & 1.89 & 0.50 & 0.79 & 631983 \\
\hline 20 & 338237 & 155 & 125273 & 0.34 & 1.93 & 0.50 & 0.78 & 741264 \\
\hline
\end{tabular}


$50 \%$ virgin polycarbonate $+50 \%$ recycled polycarbonate

\begin{tabular}{|c|l|l|l|l|l|l|l|l|}
\hline $\mathbf{w t} \%$ & $\mathbf{E}_{\mathbf{m}}, \mathbf{p s i}$ & $\begin{array}{l}\mathbf{L}_{\mathbf{f}}, \\
\boldsymbol{\mu m}\end{array}$ & $\begin{array}{l}\mathbf{G}_{\mathbf{m}}, \mathbf{p s i} \\
\mathbf{u s i n g} \text { eq. } \\
\mathbf{( 1 1 )}\end{array}$ & $\begin{array}{l}\mathbf{d} / \mathbf{2 R} \\
\mathbf{u s i n g} \\
\text { eq. (12) }\end{array}$ & $\begin{array}{l}\mathbf{x} \\
\mathbf{u s i n g} \\
\mathbf{e q .} \\
\mathbf{( 1 0 )}\end{array}$ & $\begin{array}{l}\eta_{\mathbf{L}} \\
\mathbf{u s i n g} \\
\text { eq. (9) }\end{array}$ & $\begin{array}{l}\eta_{\mathrm{o}} \\
\text { from } \\
\text { the } \\
\text { slope }\end{array}$ & $\begin{array}{l}\mathbf{E}_{\mathbf{c}}, \mathbf{p s i} \\
\mathbf{u s i n g} \text { eq. } \\
\mathbf{( 8 )}\end{array}$ \\
\hline 5 & 310945 & 173 & 115165 & 0.16 & 1.59 & 0.42 & 0.91 & 402074 \\
\hline 10 & 310945 & 172 & 115165 & 0.24 & 1.77 & 0.47 & 0.82 & 498201 \\
\hline 15 & 310945 & 159 & 115165 & 0.29 & 1.77 & 0.47 & 0.82 & 599749 \\
\hline 20 & 310945 & 155 & 115165 & 0.34 & 1.85 & 0.49 & 0.79 & 707191 \\
\hline
\end{tabular}




\section{Appendix D}

Storage modulus at temperatures $250 \mathrm{oC}$ and $260 \mathrm{oC}$ :

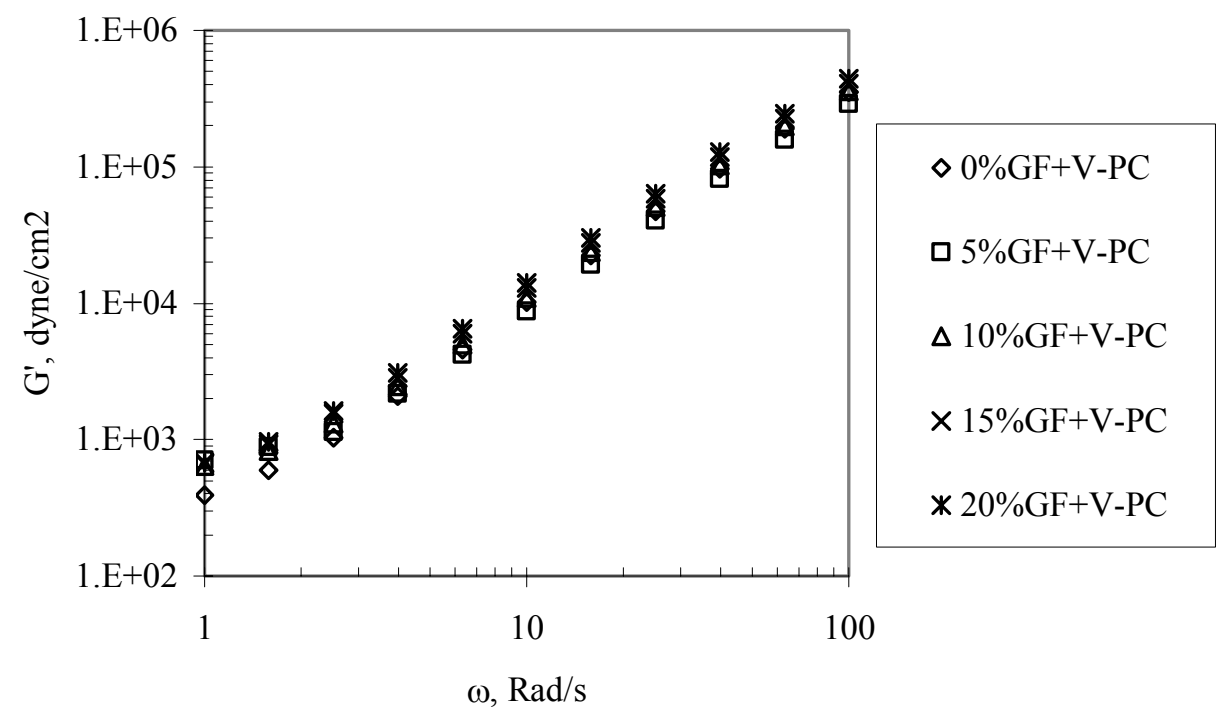

Figure E- 1 Storage modulus for virgin polycarbonate at $\mathrm{T}=250{ }^{\circ} \mathrm{C}$

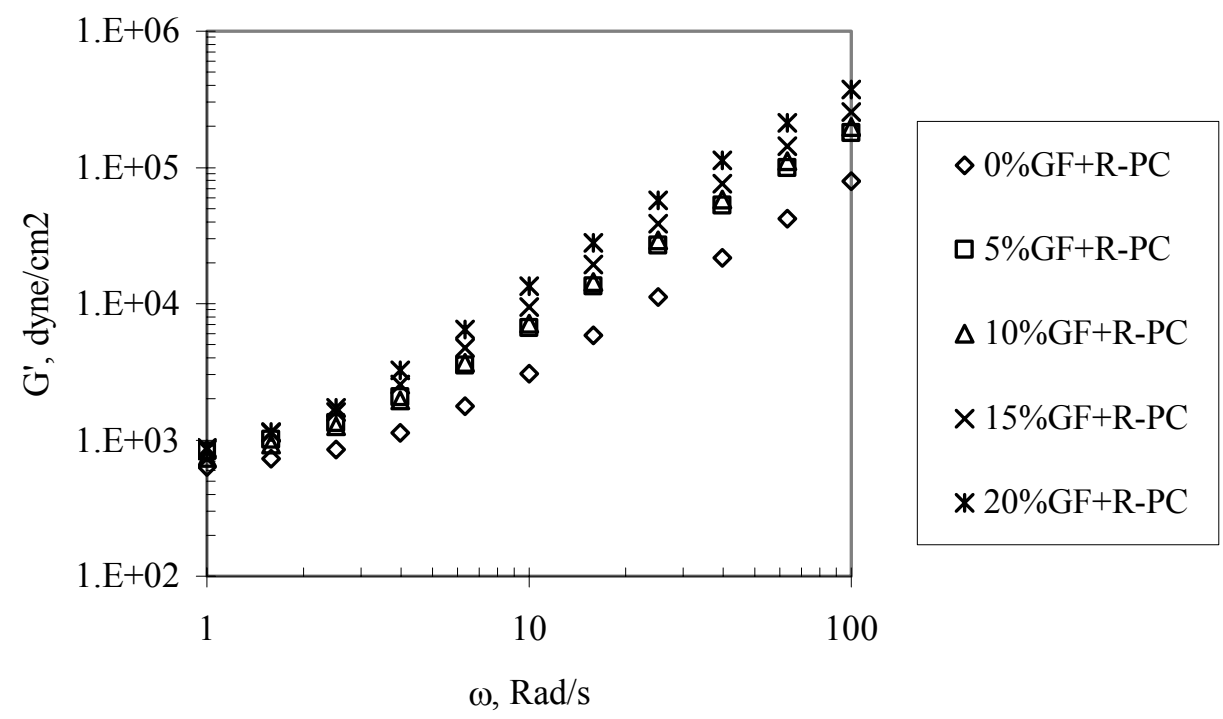

Figure E- 2 Storage modulus for recycled polycarbonate at $\mathrm{T}=250{ }^{\circ} \mathrm{C}$ 


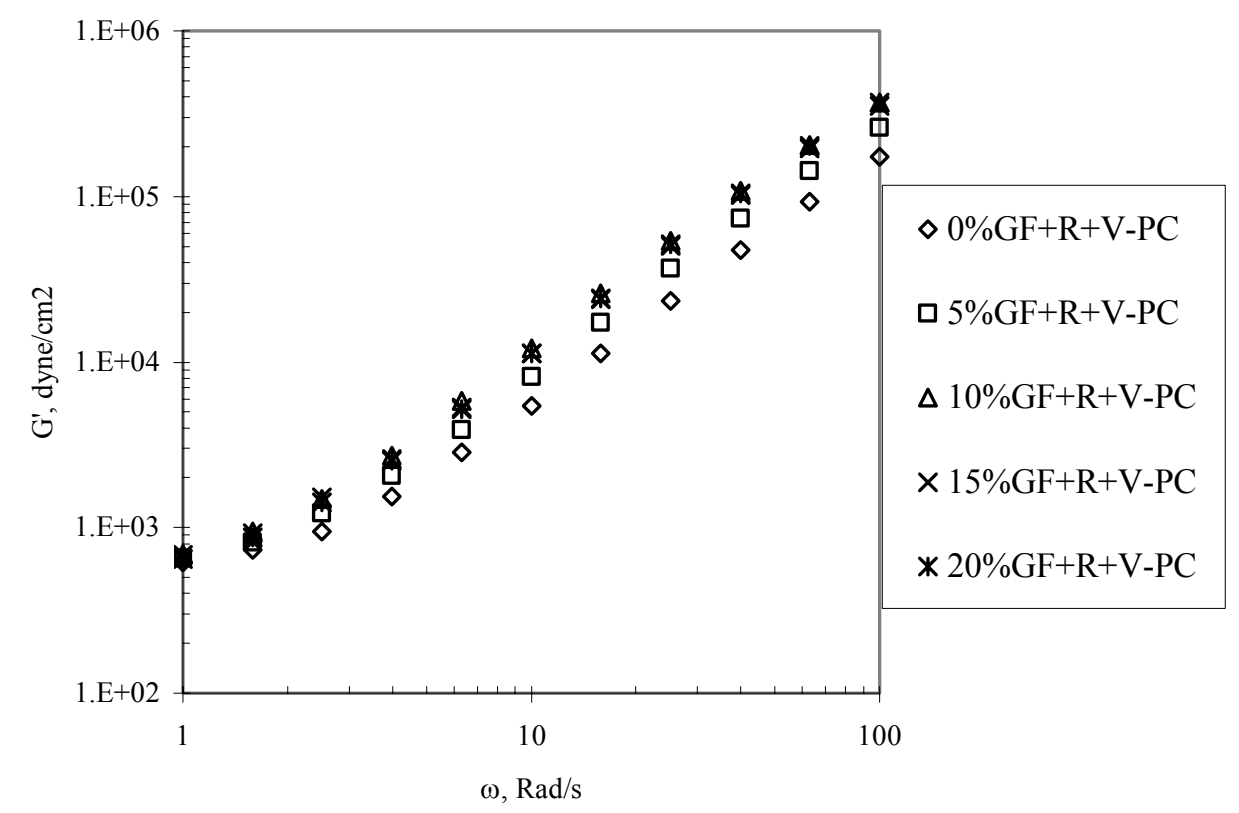

Figure E- 3 Storage modulus for 50\% virgin $\mathrm{PC}+50 \%$ recycled $\mathrm{PC}$ at $\mathrm{T}=250{ }^{\circ} \mathrm{C}$

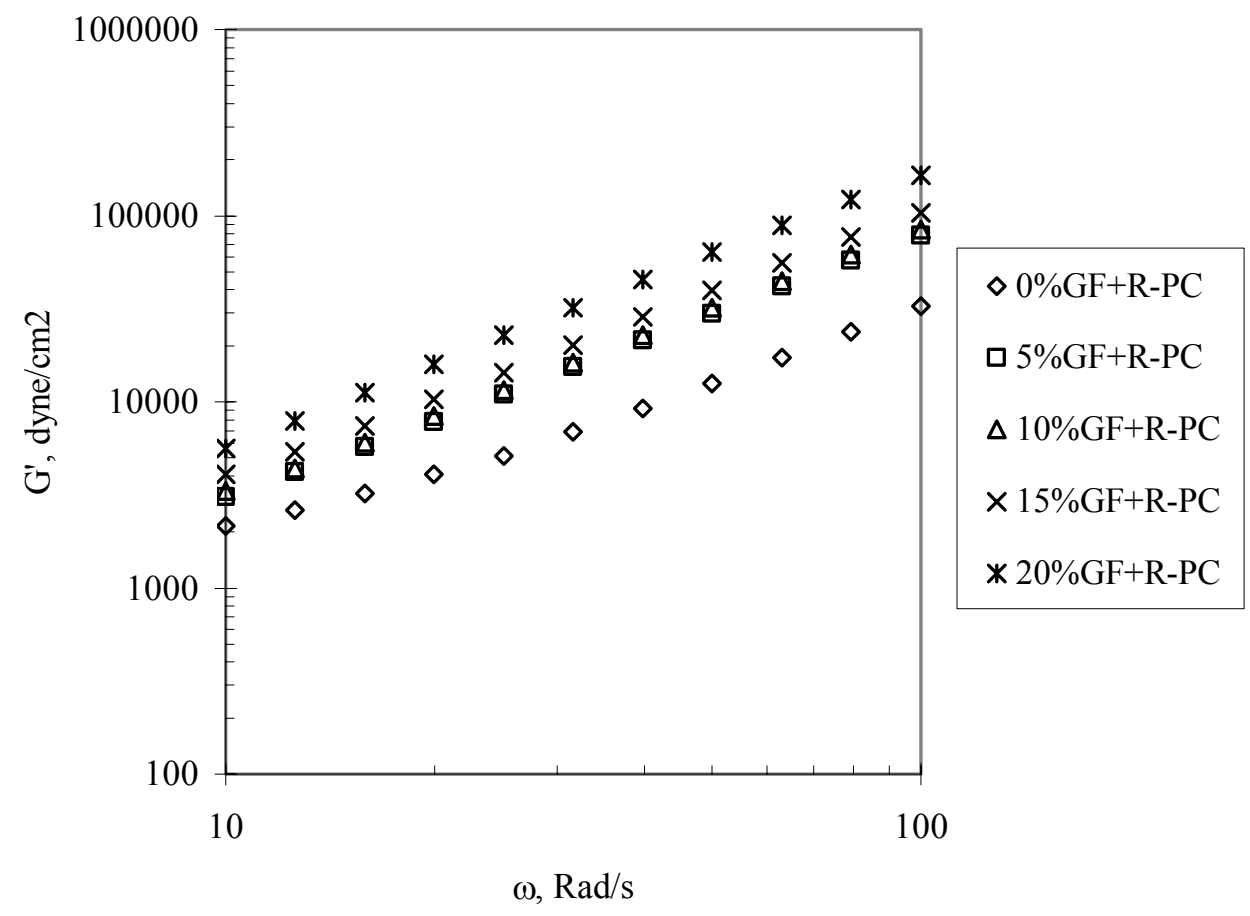

Figure E- 4 Storage modulus for recycled polycarbonate at $\mathrm{T}=260{ }^{\circ} \mathrm{C}$ 


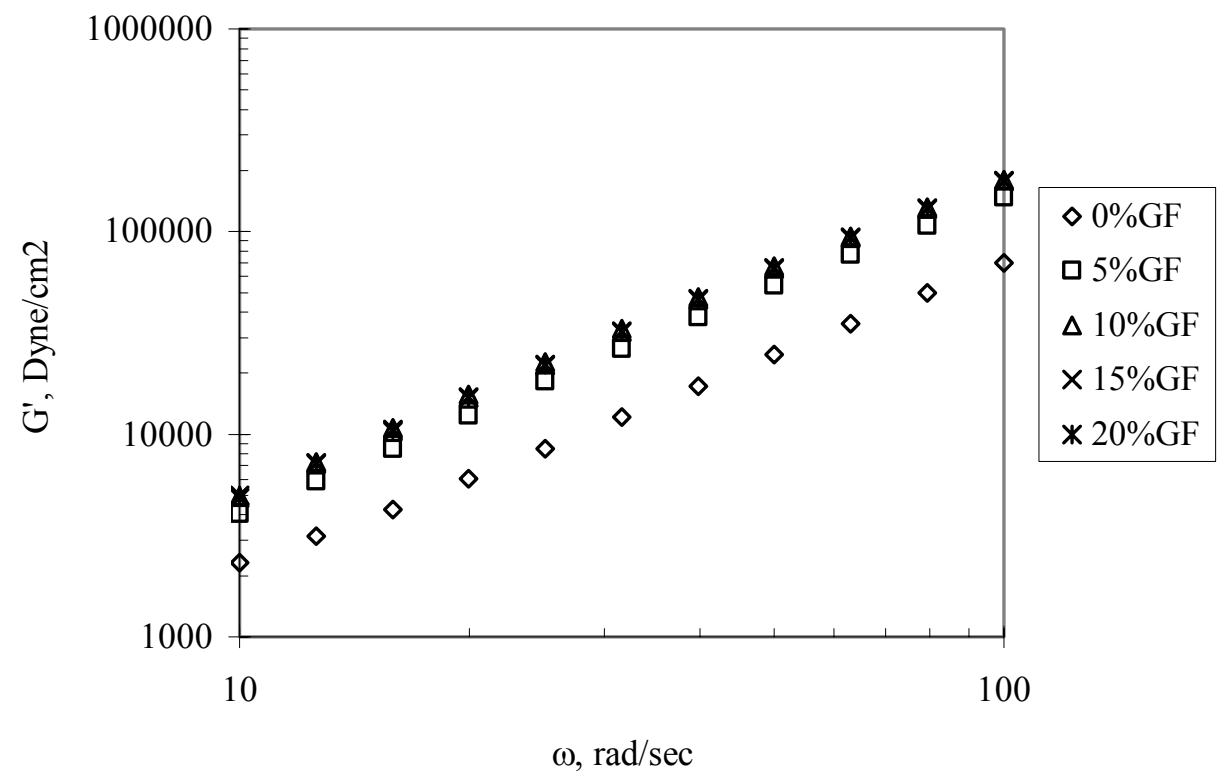

Figure E- 5 Storage modulus for $50 \%$ virgin $\mathrm{PC}+50 \%$ recycled $\mathrm{PC}$ at $\mathrm{T}=260{ }^{\circ} \mathrm{C}$

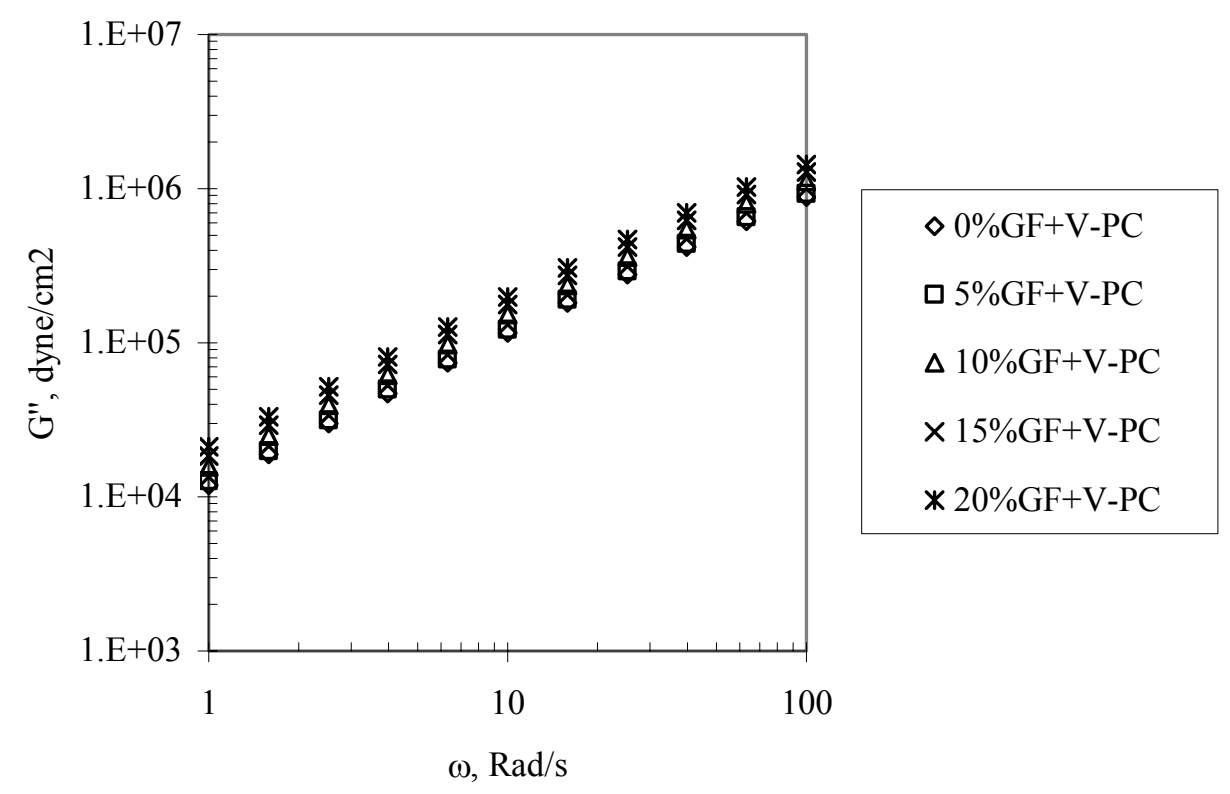

Figure E- 6 Loss modulus for virgin polycarbonate at $\mathrm{T}=250{ }^{\circ} \mathrm{C}$ 


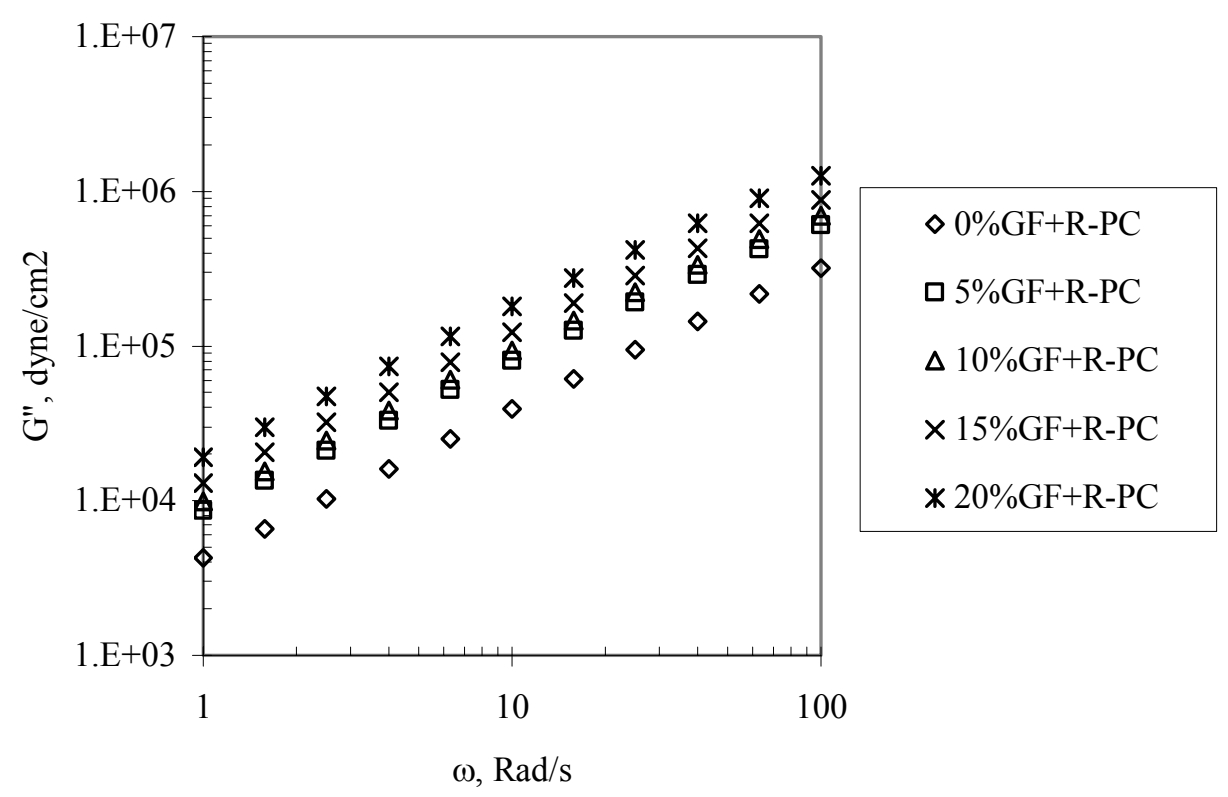

Figure E- 7 Loss modulus for recycled polycarbonate at $\mathrm{T}=250{ }^{\circ} \mathrm{C}$

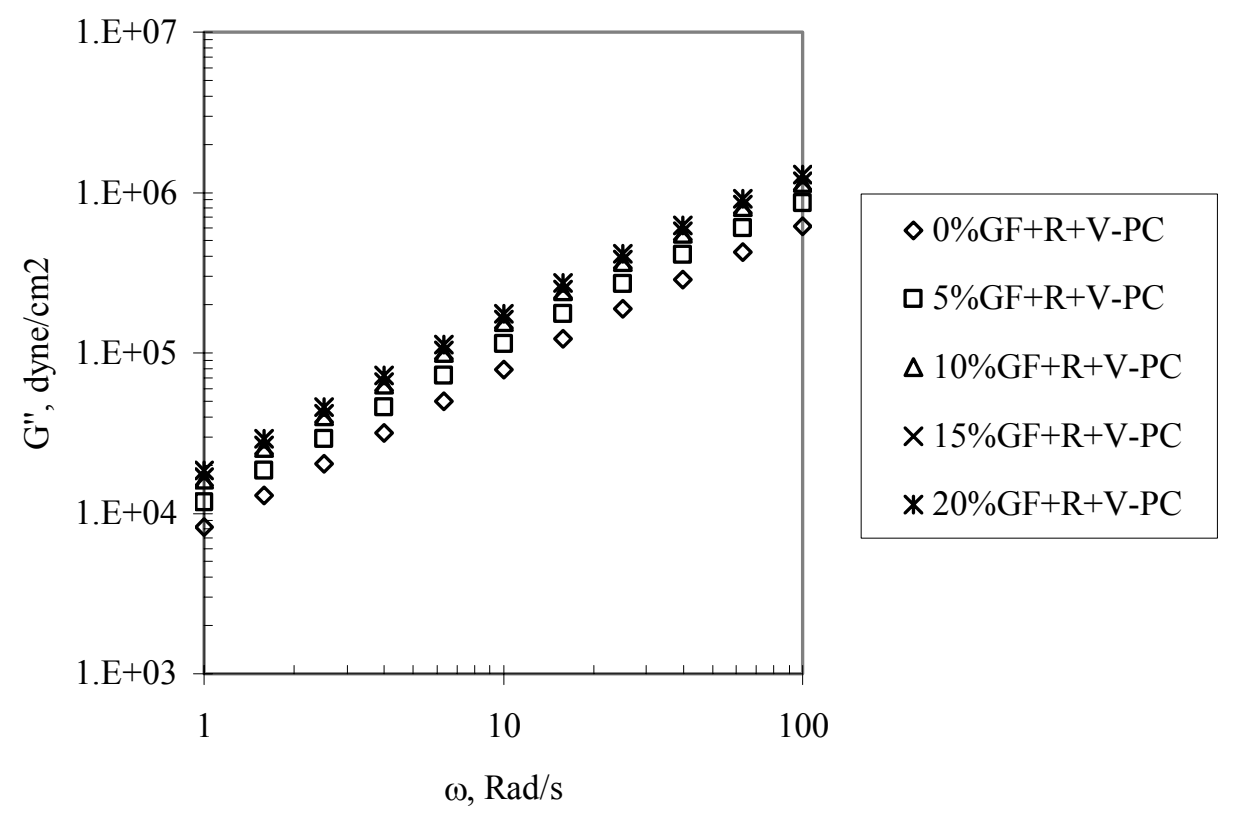

Figure E- 8 Loss modulus for $50 \%$ virgin $\mathrm{PC}+50 \%$ recycled $\mathrm{PC}$ at $\mathrm{T}=250{ }^{\circ} \mathrm{C}$ 


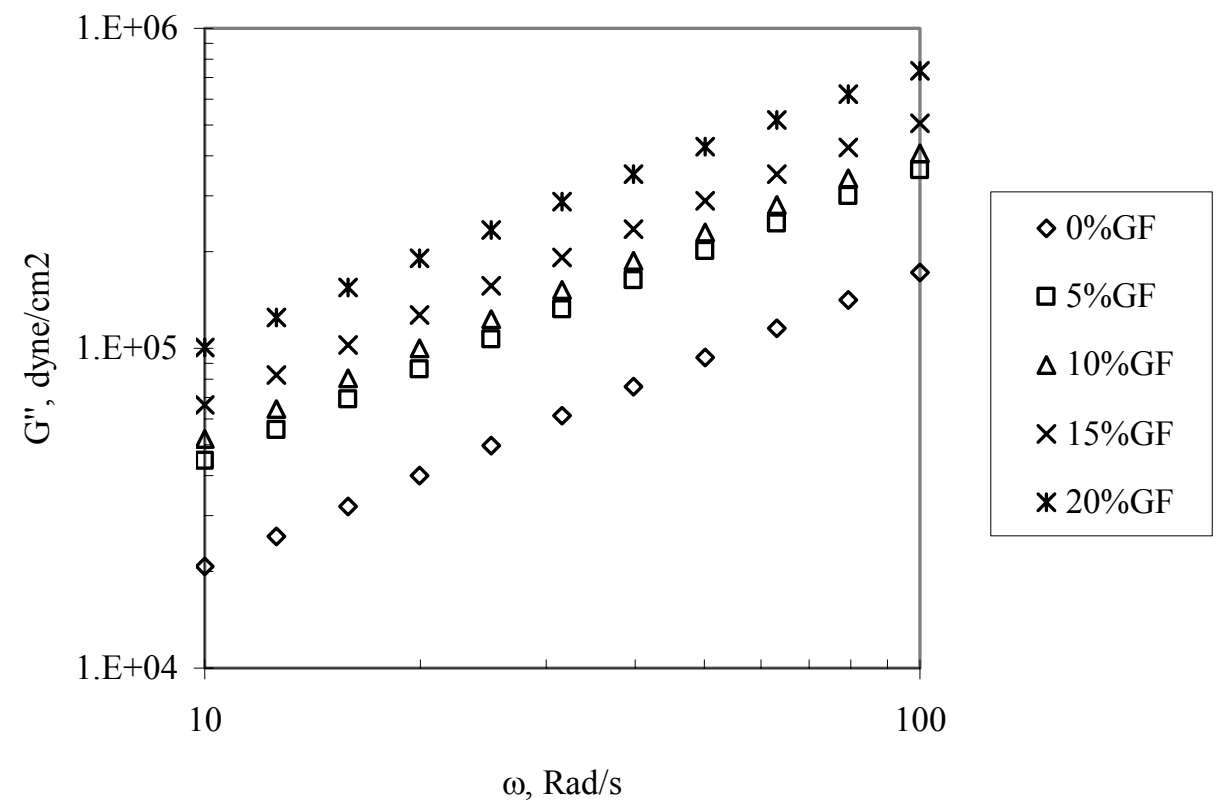

Figure E- 9 Loss modulus for recycled polycarbonate at $\mathrm{T}=260{ }^{\circ} \mathrm{C}$

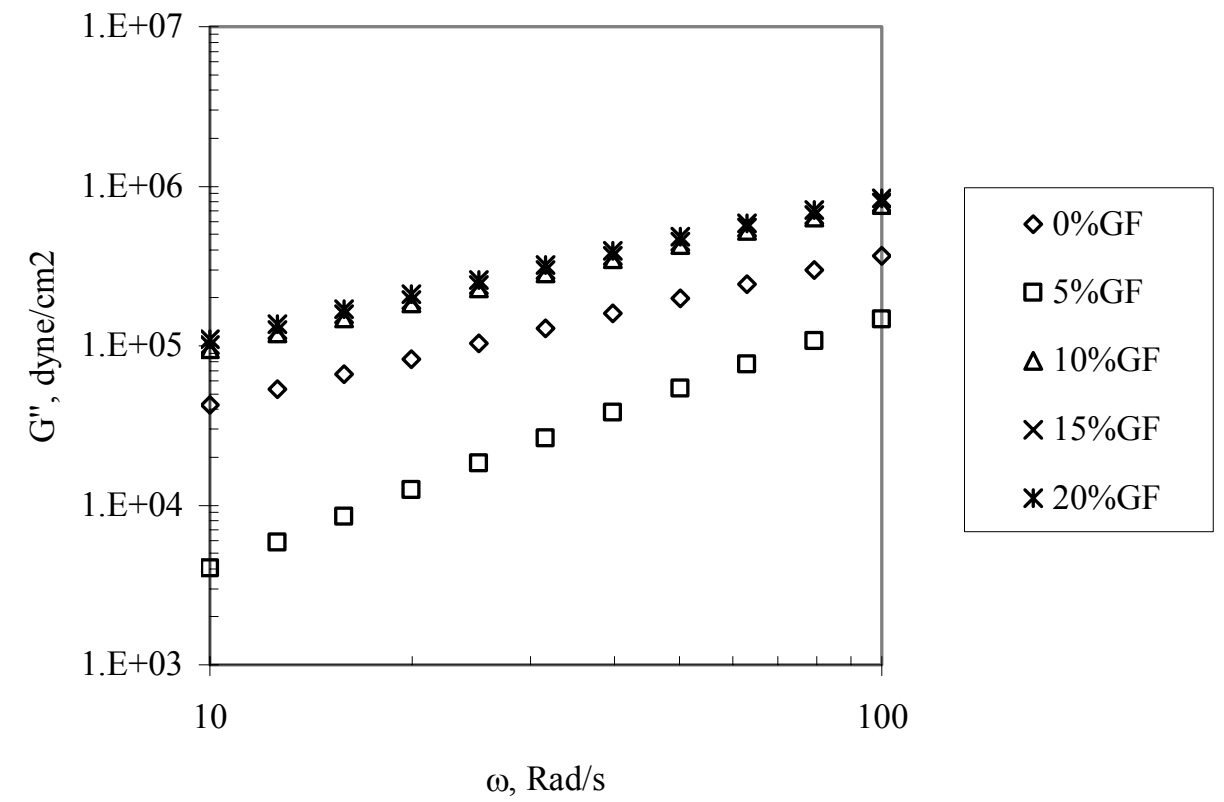

Figure E- 10 Loss modulus for 50\% virgin $\mathrm{PC}+50 \%$ recycled $\mathrm{PC}$ at $\mathrm{T}=260{ }^{\circ} \mathrm{C}$ 


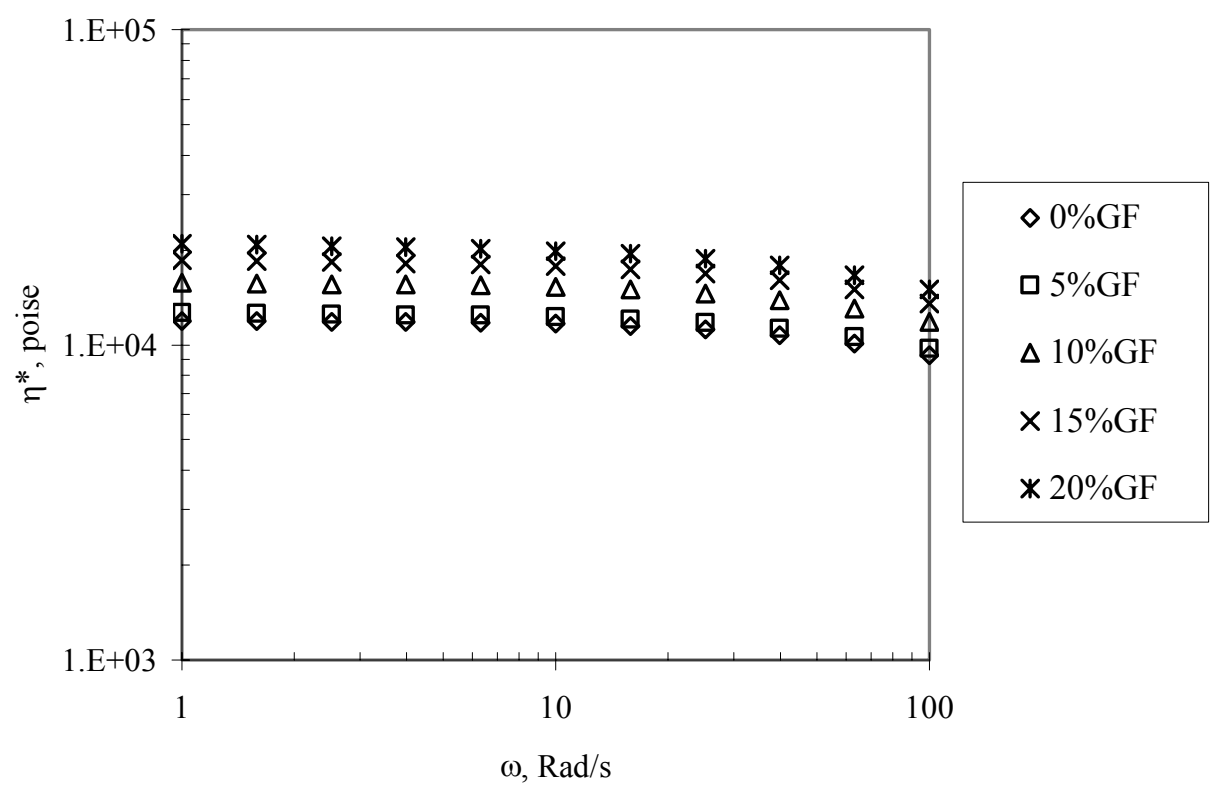

Figure E- 11 Complex viscosity for virgin polycarbonate at $\mathrm{T}=250{ }^{\circ} \mathrm{C}$

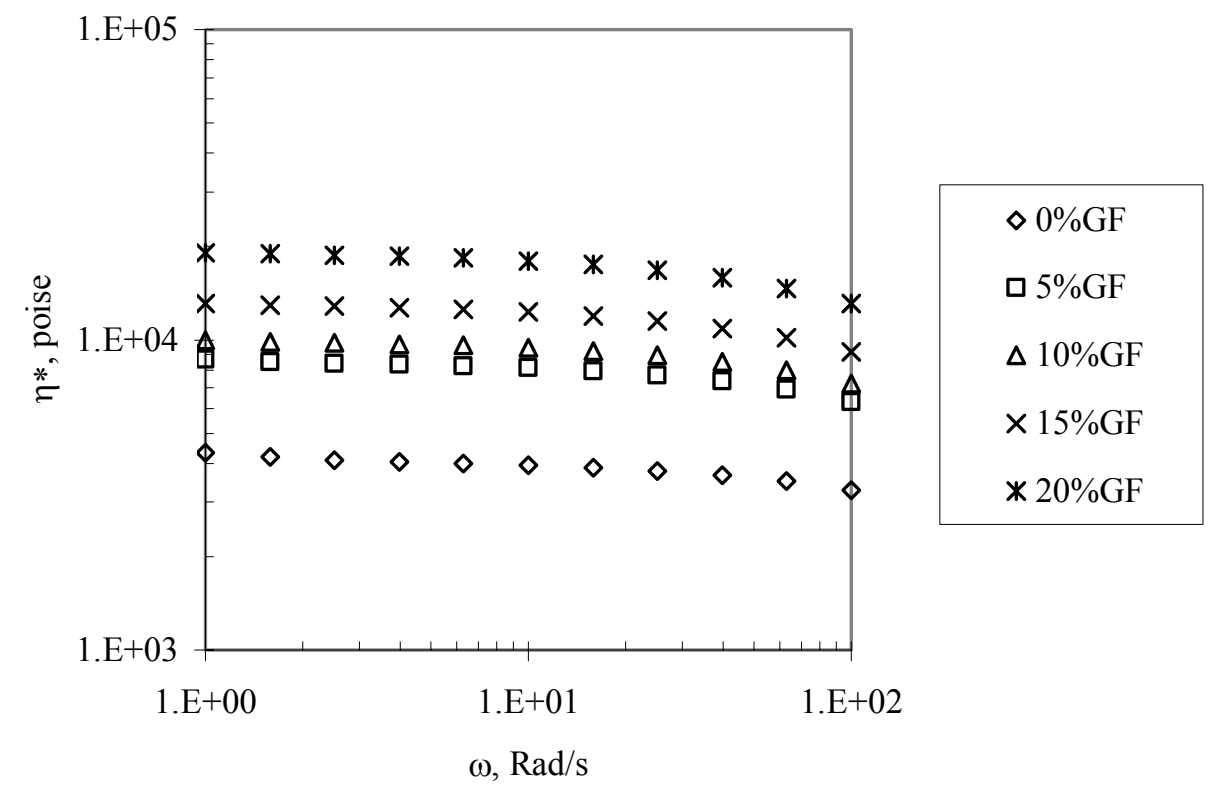

Figure E- 12 Complex viscosity for recycled polycarbonate at $\mathrm{T}=250{ }^{\circ} \mathrm{C}$ 


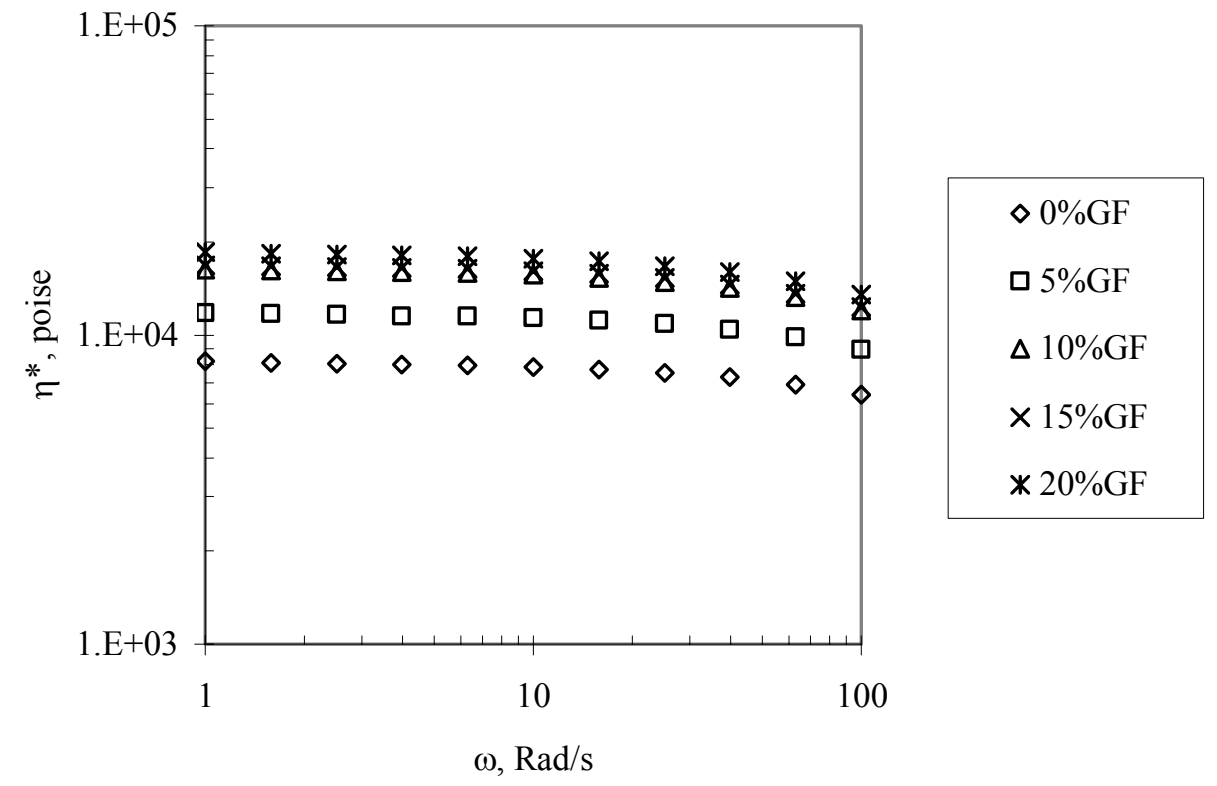

Figure E- 13 Complex viscosity for $50 \%$ virgin $\mathrm{PC}+50 \%$ recycled $\mathrm{PC}$ at $\mathrm{T}=250{ }^{\circ} \mathrm{C}$

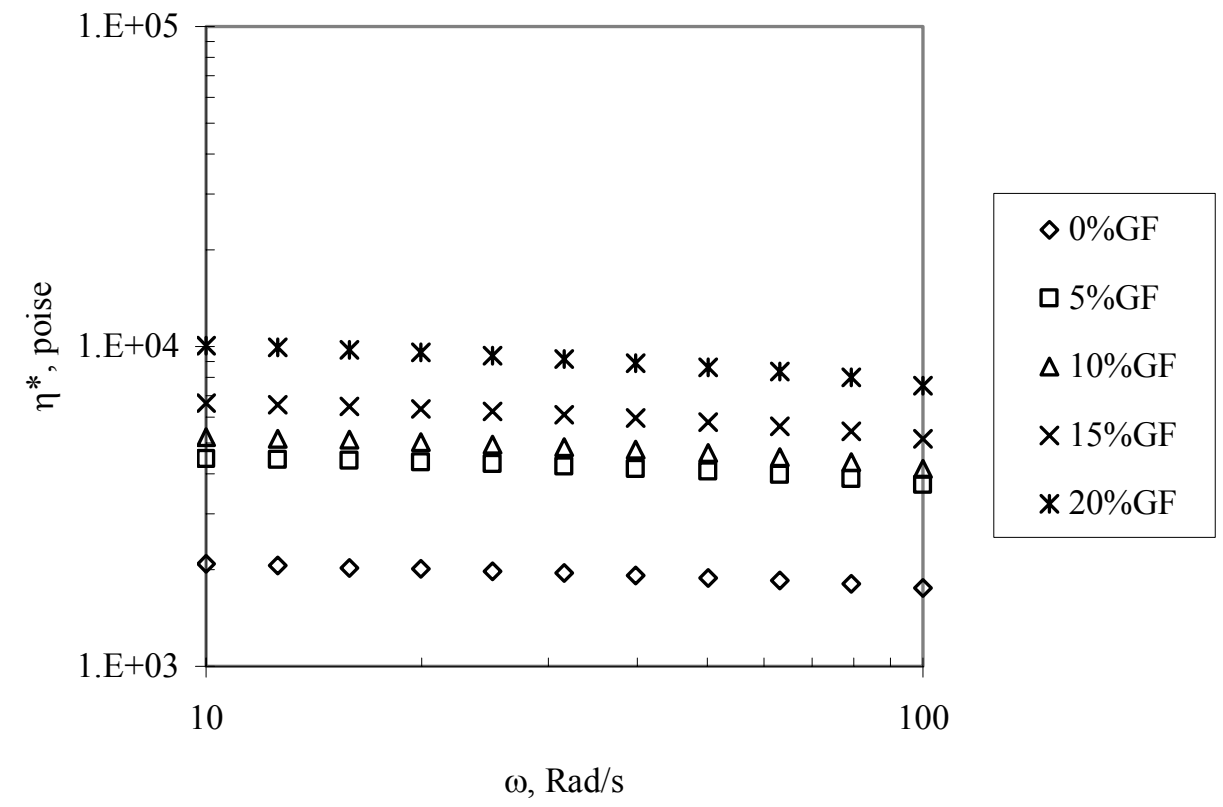

Figure E- 14 Complex viscosity for recycled polycarbonate at $\mathrm{T}=260{ }^{\circ} \mathrm{C}$ 


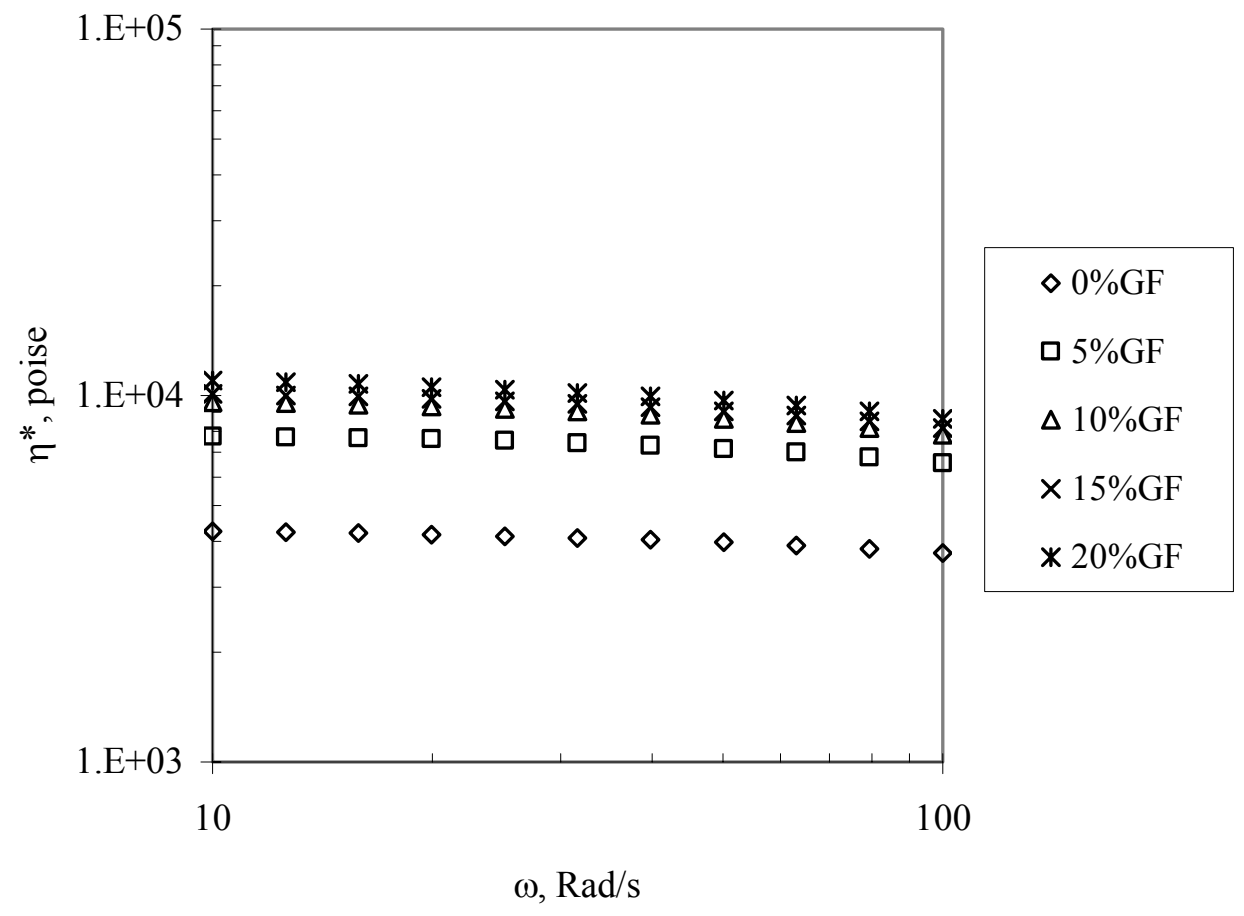

Figure E- 15 Complex viscosity for $50 \%$ virgin $\mathrm{PC}+50 \%$ recycled $\mathrm{PC}$ at $\mathrm{T}=260{ }^{\circ} \mathrm{C}$ 


\section{Appendix E}

\section{Storage modulus and loss modulus numerical simulation}

A polymeric melt is modeled as an equivalent transient network of strands linked by temporary junctions (entanglements, physical cross-links and glass fibers). A strand whose ends are linked to contiguous junctions is treated as an active one. When an end of an active strand separates from a junction, the strand is transformed into the dangling strand. When a free end of a dangling strand captures a nearby junction, the strand returns into the active state.

Separation of active strands from their junctions and merging of dangling strands with the network are thought of as thermally activated processes which occur at random times when the strands are excited by thermal fluctuations. With reference to the theory of thermally activated processes, we assume that the rate of detachment, $\Gamma$, is governed by the Eyring equation

$$
\Gamma=\gamma \exp \left(-\frac{\overline{\mathrm{v}}}{\mathrm{k}_{\mathrm{B}} \mathrm{T}}\right)
$$

where $\Gamma$ is the attempt rate, $\mathrm{k}_{\mathrm{B}}$ is Boltzmann's constant, $\mathrm{T}$ is the absolute temperature, and $\overline{\mathrm{V}} \geq 0$ is the activation energy for separation of an active strand from a temporary junction. The pre-factor $\Gamma$ in Eq. (1) is independent of activation energy, $\overline{\mathbf{V}}$, and is determined by the current temperature T only.

Confining ourselves to isothermal processes at a reference temperature $T_{o}$ and introducing the dimensionless activation energy, 


$$
\mathrm{v}=\frac{\overline{\mathrm{v}}}{\mathrm{k}_{\mathrm{B}} \mathrm{T}_{\mathrm{o}}}
$$

we find from Eq. (1) that

$$
\Gamma=\gamma \exp (-\mathrm{v})
$$

To adequately describe the time-dependent response of a melt, we suppose that different junctions are characterized by different dimensionless activation energies v. The distribution of active strands in a transient network is determined by the number of active strands per unit mass, $\mathrm{N}$, and the probability density $\mathrm{p}(\mathrm{v})$. The quantity $\mathrm{Np}(\mathrm{v}) \mathrm{dv}$ equals the number of active strands per unit mass, linked by junctions with the dimensionless activation energies, $v^{\prime}$, belonging to the interval $[\mathrm{v}, \mathrm{v}+\mathrm{dv}]$.

Separation of active strands from temporary nodes and merging of dangling strands with the network are entirely described by the function $n(t, \tau, v)$ that equals the number (per unit mass) of active strands at time $t$ linked to temporary junctions with activation energy $\mathrm{v}$ which have last been bridged to the network before instant $\tau \in[0, t]$.

The quantity $n(t, t, v)$ equals the number of active strands (per unit mass) with the energy for separation $v$ at time $t$,

$$
\mathrm{N}(\mathrm{t}, \mathrm{t}, \mathrm{v})=\mathrm{Np}(\mathrm{v})
$$

The function

$$
\varphi(\tau, \mathrm{v})=\left.\frac{\partial \mathrm{n}}{\partial \tau}(\mathrm{t}, \tau, \mathrm{v})\right|_{\mathrm{t}=\tau}
$$

is the rate of reformation for dangling chains: the amount $\varphi(\tau, \mathrm{v}) \mathrm{d} \tau$ equals the number of dangling strands (per unit mass) that merge with temporary junctions with activation 
energy $\mathrm{v}$ within the interval $[\tau, \tau+\mathrm{d} \tau]$. The quantity

$$
\frac{\partial \mathrm{n}}{\partial \tau}(\mathrm{t}, \tau, \mathrm{v}) \mathrm{d} \tau
$$

is the number of these strands that have not been broken during the interval $[\tau, t]$. The amount

$$
-\frac{\partial \mathrm{n}}{\partial \mathrm{t}}(\mathrm{t}, 0, \mathrm{v}) \mathrm{dt}
$$

is the number of active strands (per unit mass) that separate (for the first time) from the network within the interval $[t, t+d t]$, while the quantity

$$
-\frac{\partial^{2} \mathrm{n}}{\partial \mathrm{t} \partial \tau}(\mathrm{t}, \tau, \mathrm{v}) \mathrm{dtd} \tau
$$

is the number of strands (per unit mass) that have last been linked to the network within the interval $[\tau, \tau+\mathrm{d} \tau]$ and separate from the network (for the first time after merging) during the interval $[\mathrm{t}, \mathrm{t}+\mathrm{dt}]$.

The rate of separation, $\Gamma$, is determined as the ratio of the number of active strands detaching from temporary nodes per unit time to the total number of active strands. Applying this definition to active strands that have been connected with the network at the initial instant, $t=0$, and to those that merge with the network within the interval $[\tau, \tau$ $+\mathrm{d} \tau]$, we arrive at the differential equations

$$
\frac{\partial \mathrm{n}}{\partial \mathrm{t}}(\mathrm{t}, 0, \mathrm{v})=-\Gamma(\mathrm{v}) \mathrm{n}(\mathrm{t}, 0, \mathrm{v}), \quad \frac{\partial^{2} \mathrm{n}}{\partial \mathrm{t} \partial \tau}(\mathrm{t}, \tau, \mathrm{v})=-\Gamma(\mathrm{v}) \frac{\partial \mathrm{n}}{\partial \tau}(\mathrm{t}, \tau, \mathrm{v})
$$

Integration of Eqs. (5) with initial conditions (3) (where we set $t=0$ ) and (4) implies that 


$$
\begin{array}{r}
\mathrm{n}(\mathrm{t}, 0, \mathrm{v})=\mathrm{Np}(\mathrm{v}) \exp [-\Gamma(\mathrm{v}) \mathrm{t}] \\
\frac{\partial \mathrm{n}}{\partial \tau}(\mathrm{t}, \tau, \mathrm{v})=\varphi(\tau, \mathrm{v}) \exp [-\Gamma(\mathrm{v})(\mathrm{t}-\tau)]
\end{array}
$$

To exclude the function $\varphi(t, v)$ from Eq. (6), we use the identity

$$
\mathrm{n}(\mathrm{t}, \mathrm{t}, \mathrm{v})=\mathrm{n}(\mathrm{t}, 0, \mathrm{v})+\int_{0}^{\mathrm{t}} \frac{\partial \mathrm{n}}{\partial \tau}(\mathrm{t}, \tau, \mathrm{v}) \mathrm{d} \tau
$$

Substitution of expressions (3) and (6) into Eq. (7) results in

$$
\mathrm{Np}(\mathrm{v})=\mathrm{Np}(\mathrm{v}) \exp [-\Gamma(\mathrm{v}) \mathrm{t}]+\int_{0}^{\mathrm{t}} \varphi(\tau, \mathrm{v}) \exp [-\Gamma(\mathrm{v})(\mathrm{t}-\tau)] \mathrm{d} \tau
$$

The solution of the linear integral equation (8) reads

$$
\varphi(\mathrm{t}, \mathrm{v})=\mathrm{N} \Gamma(\mathrm{v}) \mathrm{p}(\mathrm{v})
$$

It follows from this equality and Eq. (6) that

$$
\frac{\partial \mathrm{n}}{\partial \tau}(\mathrm{t}, \tau, \mathrm{v})=\mathrm{N} \Gamma(\mathrm{v}) \mathrm{p}(\mathrm{v}) \exp [-\Gamma(\mathrm{v})(\mathrm{t}-\tau)]
$$

Equations (6) and (9) entirely determine the kinetics of rearrangement of strands in a transient network.

The conventional assumptions are adopted that (i) the excluded-volume effect and other multi-chain effects are screened for an individual chain by surrounding macromolecules, (ii) the energy of interaction between strands can be taken into account with the help of the incompressibility condition (Tanaka and Edwards, 1992), and (iii) the macro-strain is transmitted unchanged to active strands by surrounding macromolecules. We also accept the affinity hypothesis that disregards thermal oscillations of junctions 
and presumes that the strain tensor for the motion of junctions at the micro-level coincides with the strain tensor for the motion of appropriate points of a polymeric melt at the macro-level (Yamamoto, 1956).

At isothermal deformation with small strains, a strand in an equivalent network is treated as isotropic incompressible media. The strain energy of an active strand, $\mathrm{w}_{\mathrm{o}}(\mathrm{t})$, at an arbitrary instant $\mathrm{t} \geq 0$ is determined by the conventional formula

$$
\mathrm{w}_{\mathrm{o}}(\mathrm{t})=\mu \hat{\mathrm{e}}^{\prime}(\mathrm{t}): \hat{\mathrm{e}}^{\prime}(\mathrm{t})
$$

where $\mu$ is the average shear modulus per strand, $\hat{\mathrm{e}}(\mathrm{t})$ is the strain tensor for transition from the reference (stress-free) state of the strand to its deformed state at time $t$, and the prime stands for the deviatoric component of a tensor.

A conventional hypothesis is accepted that stress in a dangling strand totally relaxes before this strand captures a new junction (Tanaka and Edwards, 1992). This implies that the stress-free state of an active strand that merges with the network at time $\tau$ $\geq 0$ coincides with the deformed state of the network at that instant. The strain energy, $\overline{\mathrm{W}}_{\mathrm{o}}(\mathrm{t}, 0)$, of an active strand that has not separated from the network during the interval $[0, \mathrm{t}]$, reads

$$
\mathrm{w}(\mathrm{t}, 0)=\mu \hat{\mathrm{e}}^{\prime}(\mathrm{t}): \hat{\varepsilon}^{\prime}(\mathrm{t})
$$

where $\hat{\varepsilon}(t)$ is the strain tensor for transition from the initial (stress-free) state of the network to its actual state at time t. The mechanical energy of an active strand that has last been reformed at time $\tau \in[0, \mathrm{t}]$ is given by 


$$
\mathrm{w}(\mathrm{t}, \tau)=\mu[\hat{\varepsilon}(\mathrm{t})-\hat{\varepsilon}(\tau)]^{\prime}:[\hat{\varepsilon}(\mathrm{t})-\hat{\varepsilon}(\tau)]^{\prime}
$$

Multiplying the mechanical energy per strand by the number of active strands per unit mass and summing mechanical energies of strands linked to temporary junctions with various activation energies, we find the strain energy (per unit mass) of a network

$$
\begin{aligned}
\mathrm{W}(\mathrm{t}) & =\mu \int_{0}^{\infty}\left\{\mathrm{n}(\mathrm{t}, 0, \mathrm{v}) \hat{\varepsilon}^{\prime}(\mathrm{t}): \hat{\varepsilon}^{\prime}(\mathrm{t})\right. \\
& \left.+\int_{0}^{\mathrm{t}} \frac{\partial \mathrm{n}}{\partial \tau}(\mathrm{t}, \tau, \mathrm{v})[\hat{\varepsilon}(\mathrm{t})-\hat{\varepsilon}(\tau)]^{\prime}:[\hat{\varepsilon}(\mathrm{t})-\hat{\varepsilon}(\tau)]^{\prime} \mathrm{d} \tau\right\} \mathrm{dv}
\end{aligned}
$$

Differentiation of Eq. (10) with respect to time implies that

$$
\frac{\mathrm{dW}}{\mathrm{dt}}(\mathrm{t})=\hat{\mathrm{A}}(\mathrm{t}): \frac{\mathrm{d} \hat{\varepsilon}^{\prime}}{\mathrm{dt}}(\mathrm{t})-\mathrm{Y}(\mathrm{t})
$$

where

$$
\begin{aligned}
\hat{\mathrm{A}}(\mathrm{t}) & =2 \mu \int_{0}^{\infty}\left\{\mathrm{n}(\mathrm{t}, 0, \mathrm{v}) \hat{\varepsilon}(\mathrm{t}): \hat{\varepsilon}(\mathrm{t})+\int_{0}^{\mathrm{t}} \frac{\partial \mathrm{n}}{\partial \tau}(\mathrm{t}, \tau, \mathrm{v})[\hat{\varepsilon}(\mathrm{t})-\hat{\varepsilon}(\tau)] \mathrm{d} \tau\right\}^{\prime} \mathrm{dv} \\
& \mathrm{Y}(\mathrm{t})=-\mu \int_{0}^{\infty}\left\{\frac{\partial \mathrm{n}}{\partial \tau}(\mathrm{t}, 0, \mathrm{v}) \hat{\varepsilon}^{\prime}(\mathrm{t}): \hat{\varepsilon}^{\prime}(\mathrm{t})\right. \\
& \left.+\int_{0}^{\mathrm{t}} \frac{\partial^{2} \mathrm{n}}{\partial \mathrm{t} \partial \tau}(\mathrm{t}, \tau, \mathrm{v})[\hat{\varepsilon}(\mathrm{t})-\hat{\varepsilon}(\tau) \mathrm{d} \tau]^{\prime}:[\hat{\varepsilon}(\mathrm{t})-\hat{\varepsilon}(\tau) \mathrm{d} \tau]^{\prime}\right\} \mathrm{dv}
\end{aligned}
$$

Substituting expressions (6) and (9) into Eq. (12) and using Eq. (7), we find that

$$
\mathrm{A}(\mathrm{t})=2 \mu \mathrm{N}\left\{\hat{\varepsilon}(\mathrm{t})-\int_{0}^{\mathrm{t}} \hat{\varepsilon}(\tau) \mathrm{d} \tau \int_{0}^{\infty} \Gamma(\mathrm{v}) \exp [-\Gamma(\mathrm{v})(\mathrm{t}-\tau)] \mathrm{p}(\mathrm{v}) \mathrm{dv}\right\}^{\prime}
$$


It follows from Eqs. (6) and (13) that

$$
\begin{aligned}
\mathrm{Y}(\mathrm{t}) & =\mu \int_{0}^{\infty} \Gamma(\mathrm{v})\left\{\mathrm{n}(\mathrm{t}, 0, \mathrm{v}) \hat{\varepsilon}^{\prime}(\mathrm{t}): \hat{\varepsilon}^{\prime}(\mathrm{t})\right. \\
& \left.+\int_{0}^{\mathrm{t}} \frac{\partial \mathrm{n}}{\partial \tau}(\mathrm{t}, \tau, \mathrm{v})[\hat{\varepsilon}(\mathrm{t})-\hat{\varepsilon}(\tau)]:[\hat{\varepsilon}(\mathrm{t})-\hat{\varepsilon}(\tau)] \mathrm{d} \tau\right\}^{\prime} \mathrm{dv} \geq 0
\end{aligned}
$$

For isothermal deformation of an incompressible medium, the Clausius-Duhem inequality reads

$$
Q=-\frac{d W}{d t}+\frac{1}{\rho} \hat{\sigma}^{\prime}: \frac{d \hat{\varepsilon}^{\prime}}{d t} \geq 0
$$

where $\rho$ is density, $Q$ is internal dissipation per unit mass, and $\hat{\sigma}$ is the stress tensor. Substitution of Eq. (11) into this equation implies that

$$
Q(t)=\frac{1}{\rho}\left[\hat{\sigma}^{\prime}(t)-\rho \hat{A}(t)\right]: \frac{d \hat{\varepsilon}^{\prime}}{d t}(t)+Y(t) \geq 0
$$

Because the function $\mathrm{Y}(\mathrm{t})$ is non-negative, see Eq. (15), the dissipation inequality (16) is satisfied, provided that the expression in the square brackets vanishes. This assertion together with Eq. (14) results in the constitutive equation

$$
\hat{\sigma}(\mathrm{t})=-\mathrm{P}(\mathrm{t}) \hat{\mathrm{I}}+2 \mathrm{G}\left\{\hat{\varepsilon}(\mathrm{t})-\int_{0}^{\mathrm{t}} \hat{\varepsilon}(\tau) \mathrm{d} \tau \int_{0}^{\infty} \Gamma(\mathrm{v}) \exp [-\Gamma(\mathrm{v})(\mathrm{t}-\tau)] \mathrm{p}(\mathrm{v}) \mathrm{dv}\right\}
$$

where $P(t)$ is pressure, $\hat{I}$ is the unit tensor, and $G=\rho \mu N$ is the shear modulus. Formula (17) describes the time-dependent response of an equivalent network at arbitrary threedimensional deformations with small strains. In what follows, we confine ourselves to shear tests with 


$$
\hat{\varepsilon}(t)=\varepsilon(t) e_{1} e_{2}
$$

where $\varepsilon(\mathrm{t})$ is the shear strain, and $\mathrm{e}_{\mathrm{m}}(\mathrm{m}=1,2,3)$ are unit vectors of a Cartesian frame. According to Eq. (17), the shear stress, $\sigma(t)$, reads

$$
\sigma(\mathrm{t})=2 \mathrm{G}\left\{\varepsilon(\mathrm{t})-\int_{0}^{\mathrm{t}} \varepsilon(\tau) \mathrm{d} \tau \int_{0}^{\infty} \Gamma(\mathrm{v}) \exp [-\Gamma(\mathrm{v})(\mathrm{t}-\tau)] \mathrm{p}(\mathrm{v}) \mathrm{dv}\right\}
$$

The stress--strain relations (2) and (18) involve two adjustable parameters: the instantaneous shear modulus $G$ and the characteristic rate of relaxation $\gamma$, and one material function: the distribution function $\mathrm{p}(\mathrm{v})$ for temporary junctions with various activation energies $v$.

For a standard relaxation test with

$$
\varepsilon(\mathrm{t})= \begin{cases}0, & \mathrm{t}<0 \\ \varepsilon, & \mathrm{t} \geq 0\end{cases}
$$

the stress, $\sigma$, is given by

$$
\sigma(t)=2 G \varepsilon \int_{0}^{\infty} p(v) \exp [-\gamma \exp (-v) t] d v
$$

For a standard creep test with

$$
\sigma(t)= \begin{cases}0, & \mathrm{t}<0 \\ \sigma, & \mathrm{t} \geq 0\end{cases}
$$

the strain, $\varepsilon$, is given by

$$
\varepsilon(t)=\frac{\sigma}{2 G}+\int_{0}^{\infty} p(v) z(t, v) d v
$$


where the function $\mathrm{z}(\mathrm{t}, \mathrm{v})$ reads

$$
\mathrm{z}(\mathrm{t}, \mathrm{v})=\Gamma(\mathrm{v}) \int_{0}^{\mathrm{t}} \exp [-\Gamma(\mathrm{v})(\mathrm{t}-\tau)] \varepsilon(\tau) \mathrm{d} \tau
$$

Differentiating this equation with respect to time and using Eq. (2), we find that $z(t, v)$ obeys the ordinary differential equation

$$
\frac{\partial \mathrm{z}}{\partial \mathrm{t}}(\mathrm{t}, \mathrm{v})=\gamma \exp (-\mathrm{v})[\varepsilon(\mathrm{t})-\mathrm{z}(\mathrm{t}, \mathrm{v})], \quad \mathrm{z}(0, \mathrm{v})=0
$$

where $\mathrm{v}$ is a parameter.

For an standard dynamic test with

$$
\varepsilon(\mathrm{t})=\varepsilon_{\mathrm{o}} \exp (\mathrm{i} \omega \mathrm{t})
$$

where $\varepsilon_{0}$ and $\omega$ are the amplitude and frequency of oscillations, and $i=\sqrt{-1}$, the transient complex modulus

$$
\overline{\mathrm{G}}^{*}(\mathrm{t}, \omega)=\frac{\sigma(\mathrm{t})}{2 \varepsilon(\mathrm{t})}
$$

is determined by the formula

$$
\overline{\mathrm{G}}^{*}(\mathrm{t}, \omega)=\mathrm{G}\left\{1-\int_{0 \infty} \Gamma(\mathrm{v}) \mathrm{p}(\mathrm{v}) \mathrm{dv} \int_{0}^{\mathrm{t}} \exp [-(\Gamma(\mathrm{v})+\mathrm{i} \omega) \mathrm{s}] \mathrm{ds}\right\}
$$

where $s=t-\tau$. The steady-state complex modulus $G^{*}(\omega)$ is defined as the limit of $\overline{\mathrm{G}}^{*}(\mathrm{t}, \omega)$ when $\mathrm{t}$ approaches infinity. It follows from Eq. (22) that

$$
\mathrm{G}^{*}(\omega)=\mathrm{G} \int_{0}^{\infty} \frac{\mathrm{i} \omega}{\Gamma(\mathrm{v})+\mathrm{i} \omega} \mathrm{p}(\mathrm{v}) \mathrm{dv}
$$

The steady-state storage, $\mathrm{G}^{\prime}(\omega)$, and loss, G' $(\omega)$, shear moduli read 


$$
\begin{gathered}
\mathrm{G}^{\prime}(\omega)=\mathrm{G} \sum_{\mathrm{i}=1}^{\mathrm{N}} \chi_{\mathrm{i}} \frac{\left(\omega \lambda_{\mathrm{i}}\right)^{2}}{1+\left(\omega \lambda_{\mathrm{i}}\right)^{2}} \\
\mathrm{G}^{\prime \prime}(\omega)=\mathrm{G} \sum_{\mathrm{i}=1}^{\mathrm{N}} \chi_{\mathrm{i}} \frac{\left(\omega \lambda_{\mathrm{i}}\right)}{1+\left(\omega \lambda_{\mathrm{i}}\right)^{2}}
\end{gathered}
$$

Equations (19), (20)-(21), and (23) allow (i) the adjustable parameters in the stress-strain relations to be found by matching experimental data in one of the conventional tests, and (ii) the time-dependent response of a polymeric melt to be predicted in the other tests.

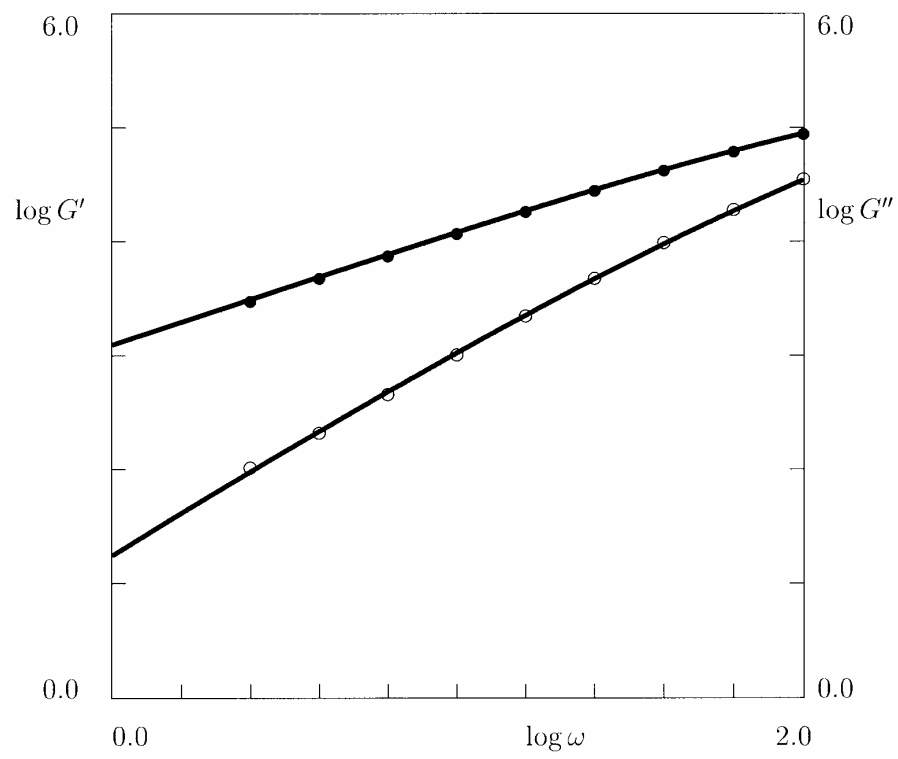

Figure E-1: The storage modulus G' MPa (unfilled circles) arid the loss modulus G" MPa (filled circles) versus frequency $\omega \mathrm{rad} / \mathrm{s}$. Symbols: experimental data for virgin PC at 250 ${ }^{\circ} \mathrm{C}$. Solid lines: results of numerical simulation 


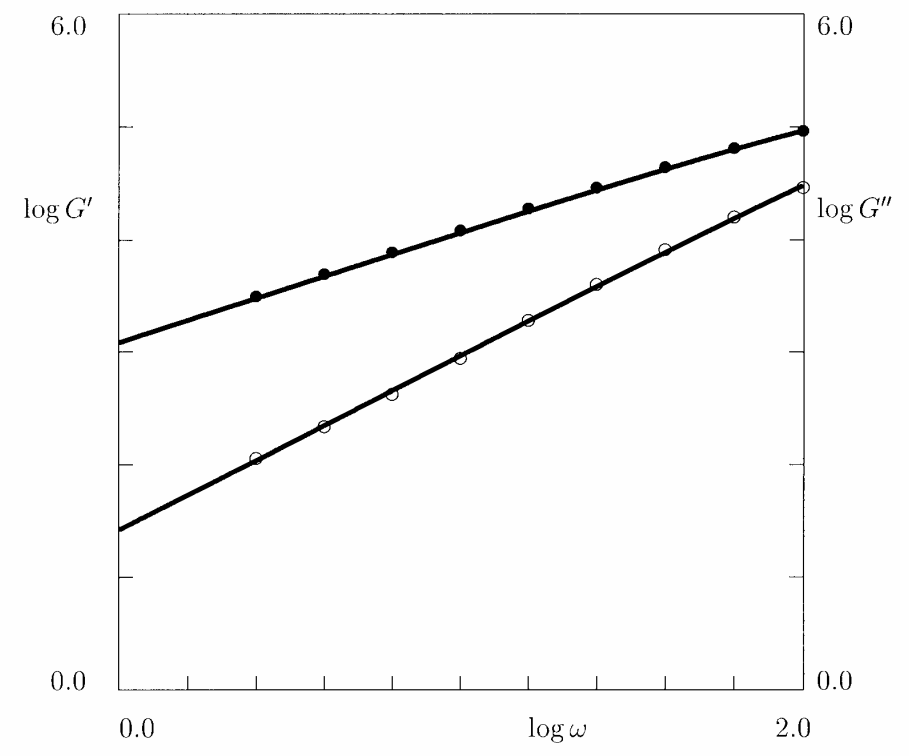

Figure E-2: The storage modulus G' MPa (unfilled circles) and the loss modulus G" MPa (filled circles) versus frequency $\omega \mathrm{rad} / \mathrm{s}$. Symbols: experimental data for virgin PC with 5 wt. $\%$ of glass fibers at $250{ }^{\circ} \mathrm{C}$. Solid lilies: results of numerical simulation

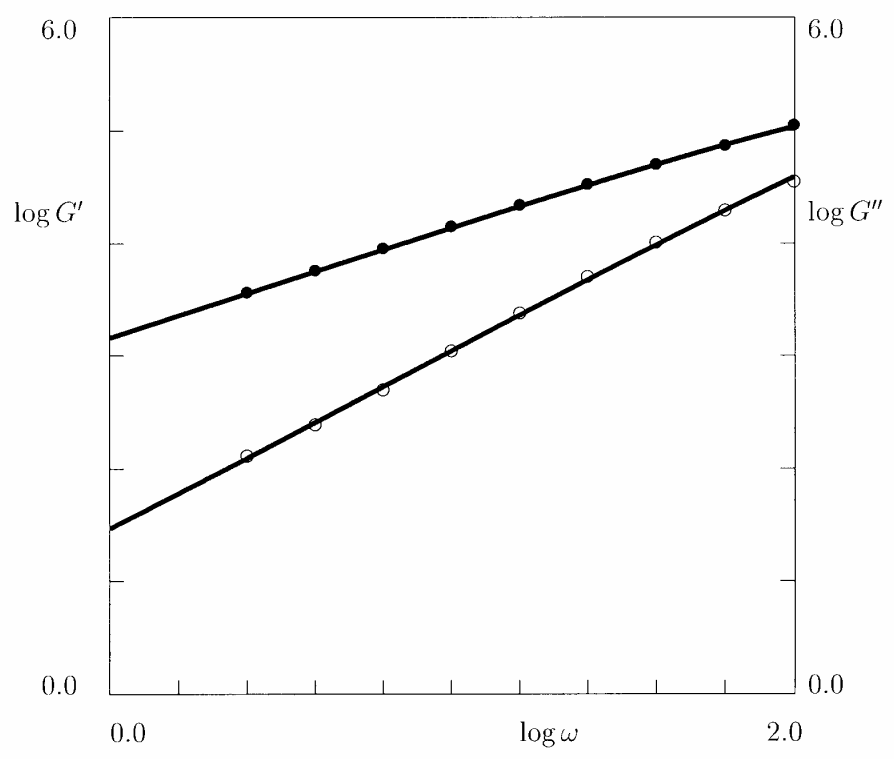

Figure E-3: The storage modulus C' MPa (unfilled circles) and the loss modulus G' MPa (filled circles) versus frequency $\omega \mathrm{rad} / \mathrm{s}$. Symbols: experimental data for virgin PC with $10 \mathrm{wt} . \%$ of glass fibers at $250^{\circ} \mathrm{C}$. Solid lines: results of numerical simulation 


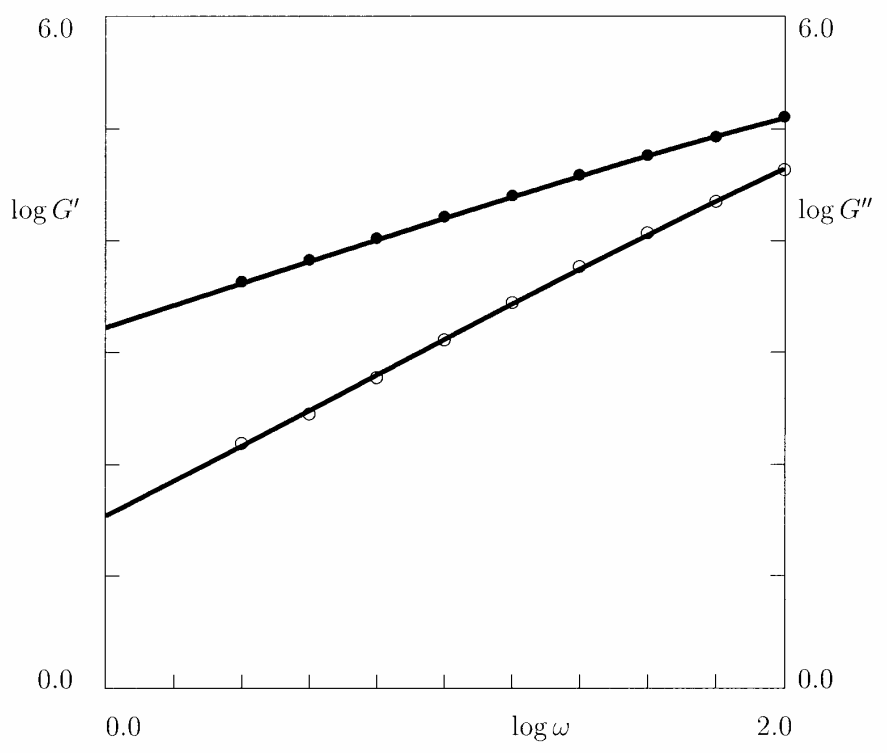

Figure E-4: The storage modulus G' MPa (unfilled circles) and the loss modulus G" MPa (filled circles) versus frequency $\omega \mathrm{rad} / \mathrm{s}$. Symbols: experimental data for virgin PC with $15 \mathrm{wt}-\%$ of glass fibers at $250^{\circ} \mathrm{C}$. Solid lines: results of numerical simulation

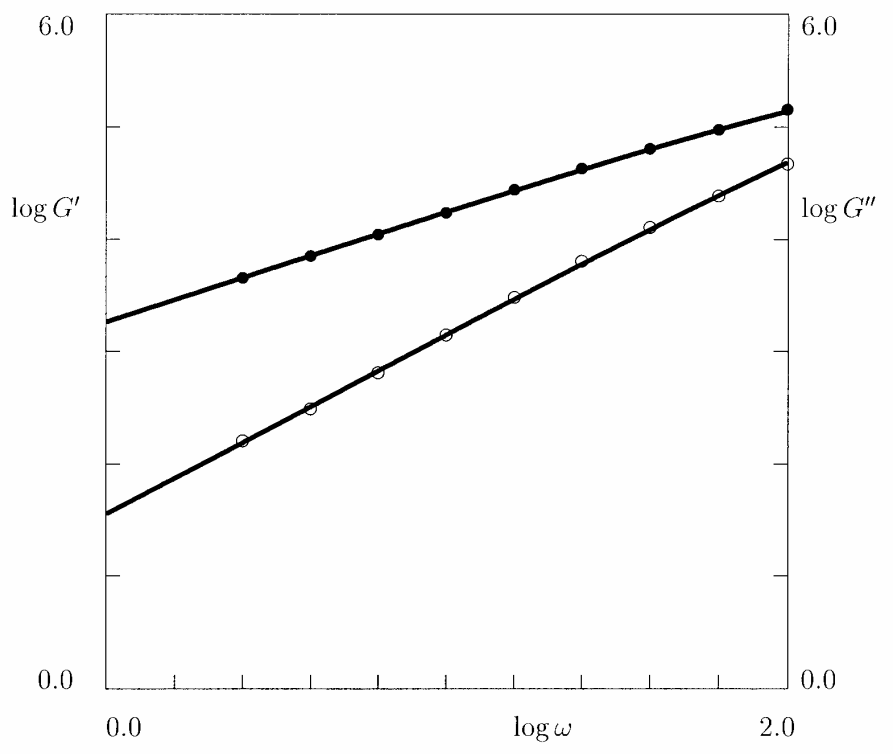

Figure E-5: The storage modulus G' MPa (unfilled circles) and the loss modulus G" MPa (filled circles) versus frequency $\omega \mathrm{rad} / \mathrm{s}$. Symbols: experimental data for virgin PC with $20 \mathrm{wt} . \%$ of glass fibers at $250^{\circ} \mathrm{C}$. Solid lines: results of numerical simulation 


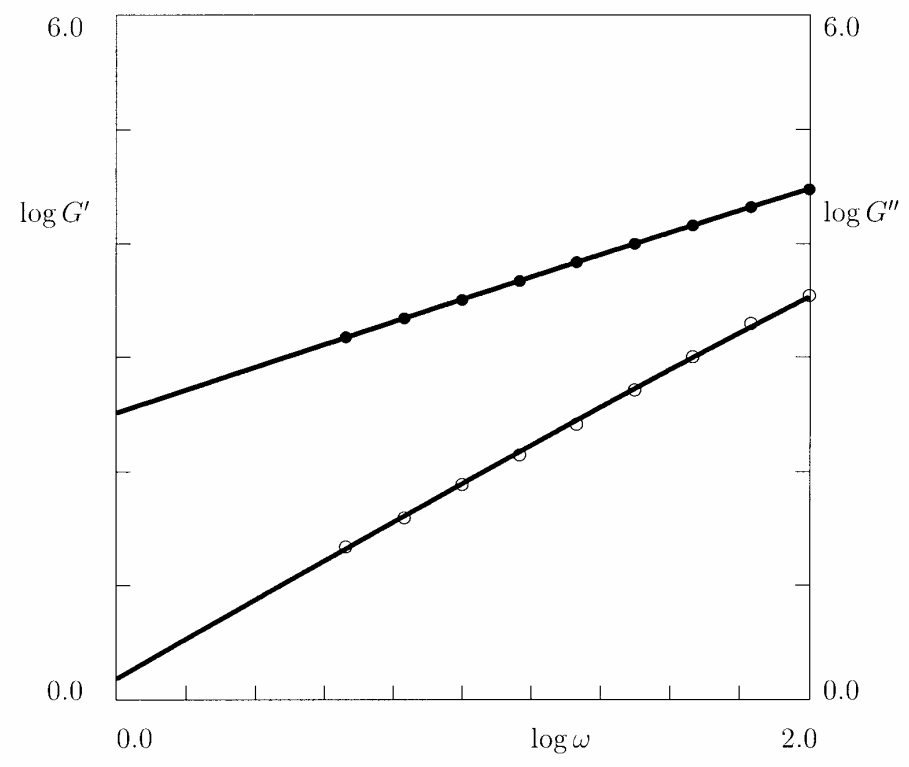

Figure E-6: The storage modulus G' MPa (unfilled circles) arid the loss modulus G" MPa (filled circles) versus frequency $\omega \mathrm{rad} / \mathrm{s}$. Symbols: experimental data for virgin PC at 290 ${ }^{\circ} \mathrm{C}$. Solid lines: results of numerical simulation

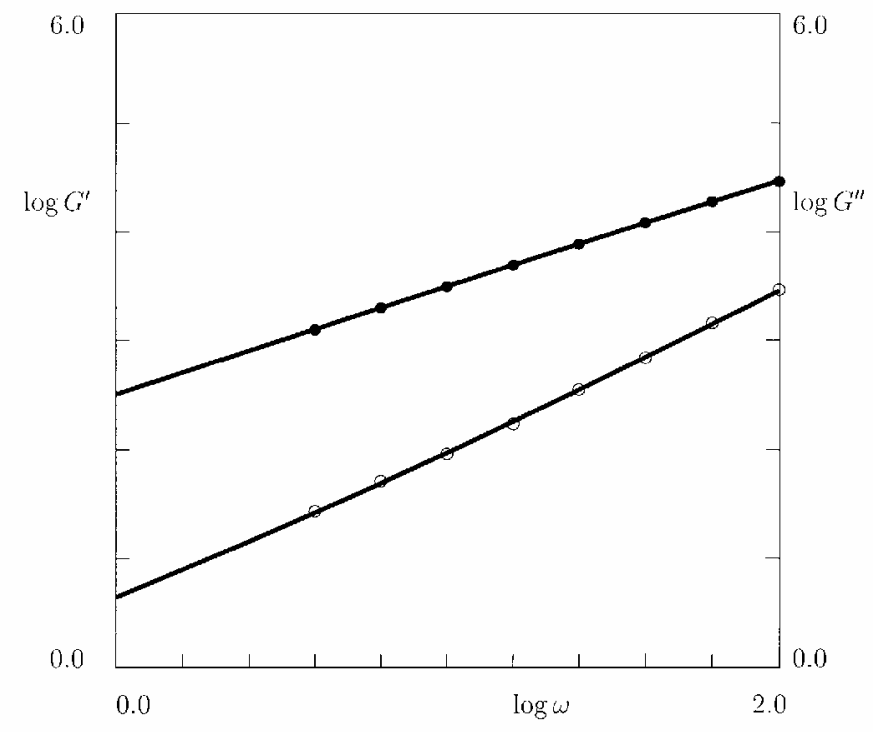

Figure E-7: The storage modulus G' MPa (unfilled circles) arid the loss modulus G" MPa (filled circles) versus frequency $\omega$ rad/s. Symbols: experimental data for virgin PC with 5 wt. $\%$ of glass fibers at $290{ }^{\circ} \mathrm{C}$. Solid lines: results of numerical simulation 


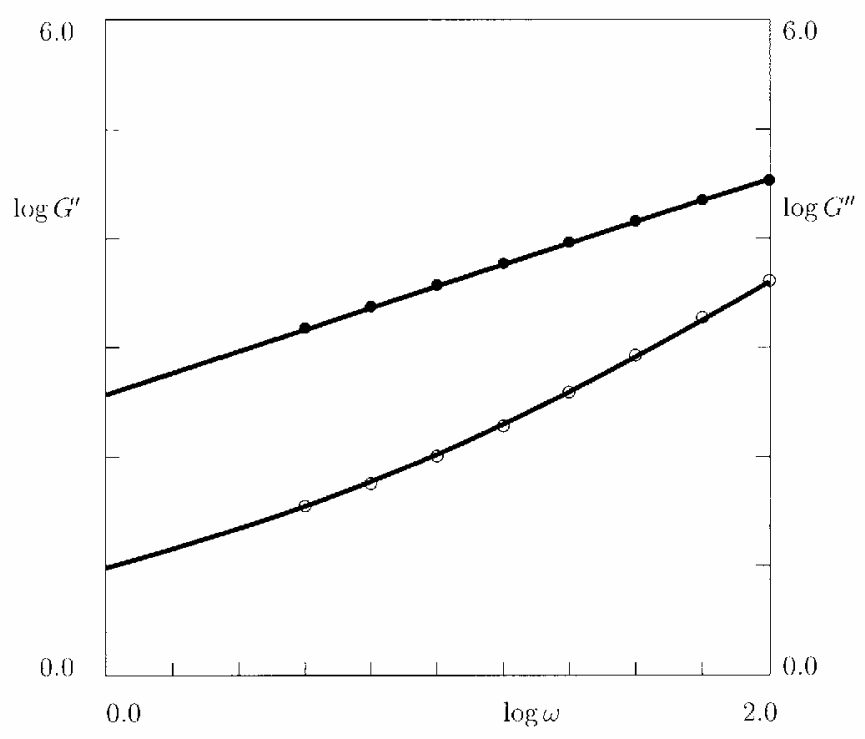

Figure E-8: The storage modulus G' MPa (unfilled circles) and the loss modulus G” MPa (filled circles) versus frequency $\omega \mathrm{rad} / \mathrm{s}$. Symbols: experimental data for virgin PC with $10 \mathrm{wt} . \%$ of glass fibers at $290^{\circ} \mathrm{C}$. Solid lines: results of numerical simulation

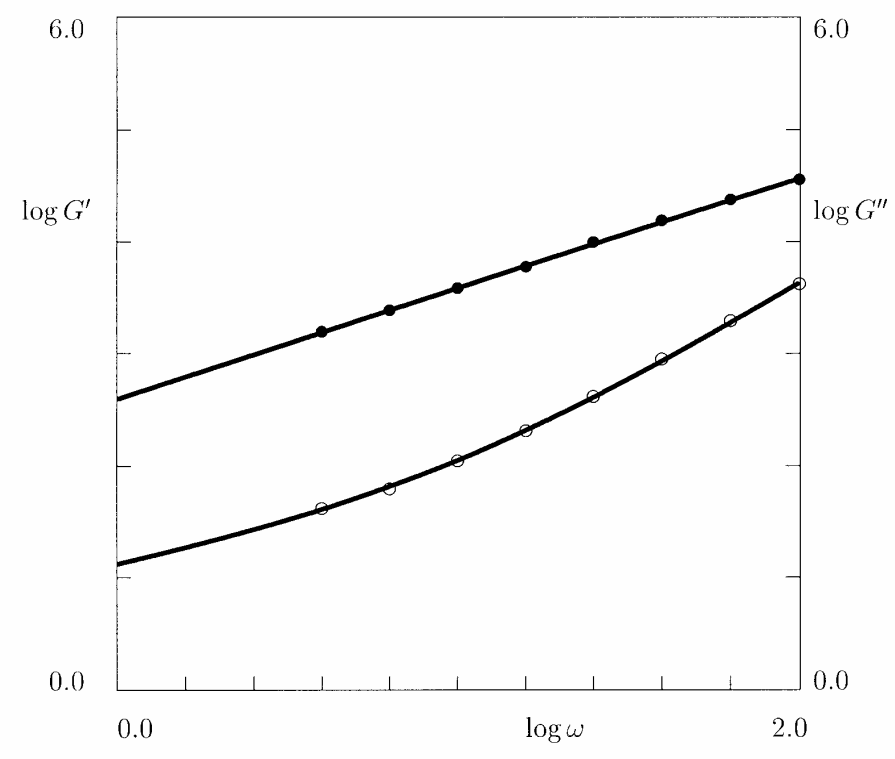

Figure E-9: The storage modulus G' MPa (unfilled circles) arid the loss modulus G” MPa (filled circles) versus frequency $\omega \mathrm{rad} / \mathrm{s}$. Symbols: experimental data for virgin PC with $15 \mathrm{wt} . \%$ of glass fibers at $290^{\circ} \mathrm{C}$. Solid lines: results of numerical simulation 


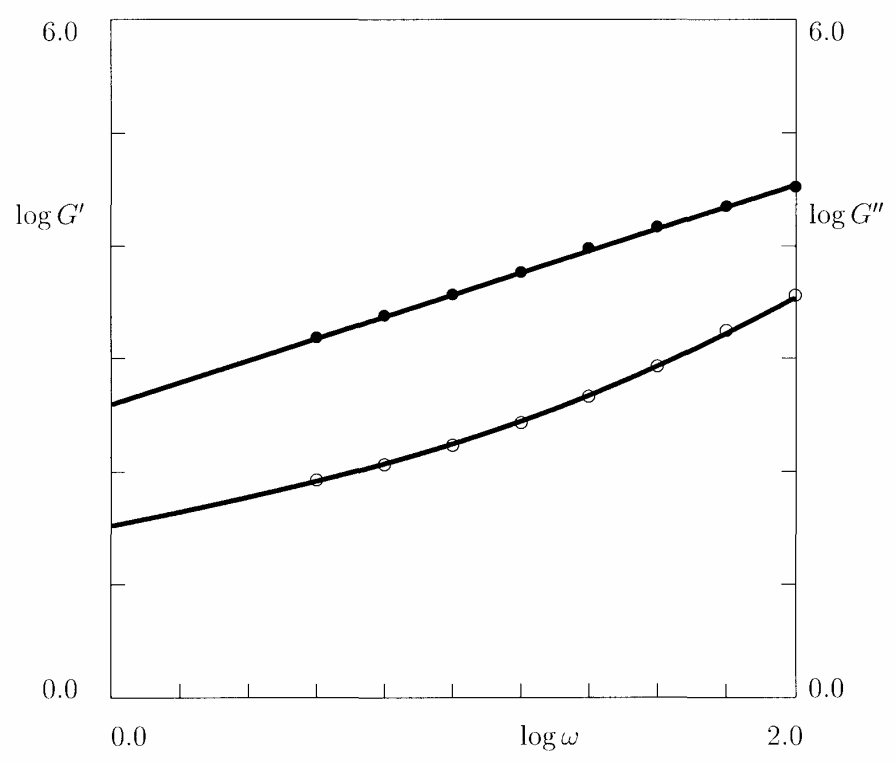

Figure E-10: The storage modulus G' MPa (unfilled circles) arid the loss modulus G' MPa (filled circles) versus frequency $\omega \mathrm{rad} / \mathrm{s}$. Symbols: experimental data for virgin PC with $20 \mathrm{wt} . \%$ of glass fibers at $290^{\circ} \mathrm{C}$. Solid lines: results of numerical simulation 


\section{Appendix F}

\section{Glossary of Terms and Definitions}

Term Definition

Additive

Aspect ratio

Average molecular weight

Bond strength

Carbon fibers

Composite the bond.
Substance added to materials, usually to improve their properties. Examples are plasticizers, flame retardants, or fillers added to plastic resins.

Ratio of length to width for a flat form, or of length to diameter for around form such as a fiber.

Molecular weight of a most typical chain found in a given plastic. There will always be a distribution of chain sizes and, hence, of molecular weights in any polymer.

Unit load, applied in tension, compression, flexure, peel, impact, cleavage, or shear, required to break an adhesive assembly, with failure occurring in or near the plane of

Fiber produced by the pyrolysis of organic precursor fibers such as rayon, polyacrylonitrile (PAN), or pitch, in an inert atmosphere.

Homogeneous material created by the synthetic assembly of two or more materials (a selected filler or reinforcing elements and compatible matrix binder) to obtain specific characteristics and properties. 
Compression molding

Compressive strength

Creep

Ductility

E-glass

Elasticity

Elongation
Technique of molding in which the molding compound (generally preheated) is placed in the heated open mold cavity and the mold is closed under pressure (usually in a hydraulic press), causing the material to flow and completely fill the cavity, with pressure being held until the material has cured.

Maximum compressive Stress a material is capable of sustaining. For materials that do not fail by a shattering fracture, the value is arbitrary, depending on the distortion allowed.

The slow movement of a plastic material with time.

Ability of a material to deform plastically before fracturing.

Family of glasses with low alkali content, usually under 2.0 percent, most suitable for use in electrical-grade laminates and glasses. Electrical properties remain more stable with these glasses due to the low alkali content. Also called electrical-grade glasses.

Property of a material by virtue of which it tends to recover its original size and shape after deformation. If the strain is proportional to the applied stress, the material is said to exhibit Hookean or ideal elasticity.

Increase in gage length of a tension specimen, usually expressed as a percentage of the original gage length. 
Elastomers

Engineering plastics

Extrusion

Fiberglass

Filler

Flexural modulus
Material that can be stretched repeatedly at room temperature to at least twice its original length and more, will return with force to its original length upon release of the stress.

Plastics, the properties of which are suitable for engineered products. These plastics are usually suitable for application up to $125^{\circ} \mathrm{C}$, well above the thermal stability of many commercial plastics. The next higher grade of plastics, called high-performance plastics, is usually suitable for product designs requiring stability of plastics above $175^{\circ} \mathrm{C}$.

Compacting of a plastic material and forcing of it through an orifice.

Individual filament made by attenuating molten glass. A continuous filament is a glass fiber of great or indefinite length; a staple fiber is a glass fiber of relatively short length.

Material, usually inert, that is added to plastics to reduce cost or to modify physical properties.

Ratio, within the elastic limit, of stress to the corresponding strain. It is calculated by drawing a tangent to the steepest initial straight-line portion of the load-deformation curve. 
Flexural strength

Gage length

Glass-transition

temperature

Izod impact test

Matrix

Melt

Modulus of elasticity
Strength of a material in bending expressed as the tensile stress of the outermost fibers of a bent test sample at the instant of failure.

Original of that portion of the specimen over which strain is measured.

Temperature at which a plastic changes from a rigid state to a softened state. Both mechanical and electrical properties degrade significantly at this point, which is usually a narrow temperature range, rather than a sharp point, as in freezing or boiling.

One of the most common ASTM (D256) tests for testing the impact strength of plastic materials.

Essentially homogeneous material in which resides the fiber system of a composite..

Molten plastic, in the melted phase of material during a molding cycle.

Ratio of unidirectional stress to the corresponding strain (slope of the line) in the linear stress-strain region below the proportional limit. For materials with no linear range, a secant line from the origin to a specified point on the stress-strain curve or a line tangent to the curve at a specified point may be used. 
Necking

Refractive index

Reinforced plastic

S-glass

Shear strength

Shore hardness

Strain
Localized reduction of the cross-sectional area of a tensile specimen that may occur during loading.

Ratio of the velocity of light in a vacuum to its velocity in a substance; also ratio of the sine of the angle of incidence to the sine of the angle of refraction.

Plastic with strength properties greatly superior to those of the base resin, resulting from the presence of reinforcements in the composition.

Glass fabric made with very high tensile strength fibers for high-performance-strength requirements

Maximum shear stress a material is capable of sustaining. In testing, the shear stress is caused by a shear or torsion load and is based on the original specimen dimensions.

Procedure for determining the indentation hardness of a material by means of a durometer. Shore designation is given to tests made with a specified durometer instrument.

Deformation resulting from a stress, measured by the ratio of the change to the total value of the dimension in which the change occurred; unit change, due to force, in the size or shape of a body referred to its 
original size or shape. Strain is non-dimensional but frequently expressed in inches per inch or centimeters per centimeter.

Stress

Tensile strength

Thermal coefficient of expansion

Thermoplastic

Viscosity

Yield strength
Unit force or component of force at a point in a body acting on a plane through the point. Stress is usually expressed in pounds per square inch.

Maximum tensile stress a material is capable of sustaining. Tensile strength is calculated from the maximum load during a tension test carded to rupture and the original cross-sectional area of the specimen.

This is a measurement of how much the length of a material will change when polymer is heated. The value given is based on $\mathrm{cm}$ as a unit.

Plastics capable of being repeatedly softened or melted by increases in temperature, or hardened by decreases in temperature. These changes are physical rather than chemical.

Measure of the resistance of a fluid to flow (usually through a specific orifice).

Lowest stress at which a material undergoes plastic deformation. Below this stress, the material is elastic; above it, the material is viscous. Also, stress at which a material exhibits a specified limiting deviation from the 
proportionality of stress to strain.

Young's modulus

ABS

HIPS

LDPE

MFI

PC

PET

PMMA
Ratio of normal stress to corresponding strain for tensile or compressive Stresses at less than the proportional limit of the material.

Acrylonitrile Butadiene Styrene

High impact Polystyrene

Low density Polyethylene

Melt flow index

Polycarbonate

Polyethylene terephthalate

Polymethyl Methacrylate Acrylic 